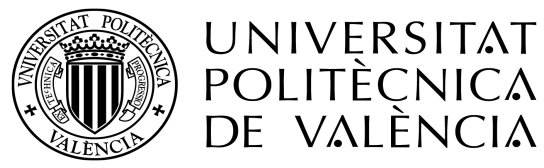

Ph.D. in CONTRol, Robotics And Industrial COMPUTING

Programa de Doctorado en Automática, Robótica e Informática INDUSTRIAL

\title{
Robust strategies for glucose control in type 1 diabetes
}

Ana Revert Tomás

Supervisors:

Jesús Picó i Marco

Jorge Bondia Company

Instituto Universitario de Automática e Informática Industrial (AI2)

Departamento de Ingeniería de Sistemas y Automática (ISA)

September 2015 

This work is partially supported by the Spanish Government under grants DPI-2007-66728, DPI-2010-20764-C02-01, DPI-2008-06880-C03-01 and FEDER-CICYT DPI2014-55276-C5-1-R. The author was also recipient of a fellowship from the Spanish Ministry of Science and Innovation (FPI BES2009-020327). 




\section{Agradecimientos}

Antes que nada tengo que dar las gracias a las dos personas que más culpa tienen de que esté escribiendo ahora estas líneas. Jorge, gracias por recibirme esa mañana hace ya varios años, contagiarme tu entusiasmo y permitirme descubrir el apasionante mundo de la investigación y de la biomedicina. Jesús, sin tu empuje nunca lo hubiera conseguido, muchas gracias por todo, de corazón.

Los otros grandes culpables son mis compis de la sala de doctorado: Alejandro L., Fàtima, Kiko, David, Gilberto, Alejandro V., Diego, Jose Luis y Gabi. No puedo imaginar mejores compañeros de viaje que vosotros. Gracias por los momentos en la sala y fuera de ella, por la ayuda, los consejos, por las risas, el padel, el yoga, los montaditos, el cafeteo... Os deseo toda la suerte porque os la merecéis.

Gracias a mis padres por ser tan entregados y por vivir mis éxitos y fracasos más intensamente que si fueran suyos propios. $\mathrm{Lu}$, gracias por ayudarme siempre en mis momentos de indecisión, sabes como valoro tus sabios consejos. Dani, gracias por estar siempre a mi lado incluso cuando estás lejos, por ser mi motor y ordenarme cuando me desordeno.

Finalmente, quiero dedicar esta tesis a mis abuelos, a los que ya no están y también y en especial a Consue, por estar siempre orgullosa de mí, pase lo que pase, y porque sé que será la persona que con más interés y cariño pasará las páginas de esta tesis, aunque no entienda ni una palabra.

¡Gracias a todos! 



\section{Abstract}

Type 1 diabetes mellitus is a chronic and incurable disease that affects millions of people all around the world. Its main characteristic is the destruction (totally or partially) of the beta cells of the pancreas. These cells are in charge of producing insulin, main hormone implied in the control of blood glucose. Keeping high levels of blood glucose for a long time has negative health effects, causing different kinds of complications. For that reason patients with type 1 diabetes mellitus need to receive exogenous.

Since 1921 when insulin was first isolated to be used in humans and first glucose monitoring techniques were developed, many advances have been done in clinical treatment with insulin. Currently 2 main research lines focused on improving the life quality of diabetic patients are opened. The first one is concentrated on the research of stem cells to replace damaged beta cells and the second one has a more technological orientation. This second line focus on the development of new insulin analogs to allow emulating with higher fidelity the endogenous pancreas secretion, the development of new noninvasive continuous glucose monitoring systems and insulin pumps capable of administering different insulin profiles and the use of decision-support tools and telemedicine. The most important challenge the scientific community has to overcome is the development of an artificial pancreas, that is, to develop algorithms that allow an automatic control of blood glucose.

The main difficulty avoiding a tight glucose control is the high variability found in glucose metabolism. This fact is especially important during meal compensation. This variability, together with the delay in subcutaneous 
insulin absorption and action causes controller overcorrection that leads to late hypoglycemia (the most important acute complication of insulin treatment).

The proposals of this work pay special attention to overcome these difficulties. In that way, interval models are used to represent the patient physiology and to be able to take into account parametric uncertainty. This type of strategy has been used in both the open loop proposal for insulin dosage and the closed loop algorithm. Moreover the idea behind the design of this last proposal is to avoid controller overcorrection to minimize hypoglycemia while adding robustness against glucose sensor failures and over/under- estimation of meal carbohydrates. The algorithms that have been proposed have been validated both in simulation and in clinical trials. 


\section{Resumen}

La diabetes mellitus tipo 1 es una enfermedad crónica e incurable que afecta a millones de personas en todo el mundo. Se caracteriza por una destrucción total o parcial de las células beta del páncreas. Estas células son las encargadas de producir la insulina, hormona principal en el control de glucosa en sangre. Valores altos de glucosa en la sangre mantenidos en el tiempo afectan negativamente a la salud, provocando complicaciones de diversa índole. Es por eso que los pacientes con diabetes mellitus tipo 1 necesitan recibir insulina de forma exógena.

Desde que se consiguiera en 1921 aislar la insulina para poder utilizarla en clínica humana, y se empezaran a desarrollar las primeras técnicas de monitorización de glucemia, se han producido grandes avances en el tratamiento con insulina. Actualmente, las líneas de investigación que se están siguiendo en relación a la mejora de la calidad de vida de los pacientes diabéticos, tienen fundamentalmente 2 vertientes: una primera que se centra en la investigación en células madre para la reposición de las células beta y una segunda vertiente de carácter más tecnológico. Dentro de esta segunda vertiente, están abiertas varias líneas de investigación, entre las que se encuentran el desarrollo de nuevos análogos de insulina que permitan emular más fielmente la secreción endógena del páncreas, el desarrollo de monitores continuos de glucosa no invasivos y de bombas de insulina capaces de administrar distintos perfiles de insulina y la inclusión de sistemas de ayuda a la decisión y telemedicina. El mayor reto al que se enfrentan los investigadores es 
el de conseguir desarrollar un páncreas artificial, es decir, desarrollar algoritmos que permitan disponer de un control automático de la glucosa.

La principal barrera que se encuentra para conseguir un control riguroso de la glucosa es la alta variabilidad que presenta su metabolismo. Esto es especialmente significativo durante la compensación de las comidas. Esta variabilidad junto con el retraso en la absorción y actuación de la insulina administrada de forma subcutánea favorece la aparición de hipoglucemias tardías (complicación aguda más importante del tratamiento con insulina) a consecuencia de la sobreactuación del controlador.

Las propuestas presentadas en este trabajo hacen especial hincapié en sobrellevar estas dificultades. Así, se utilizan modelos intervalares para representar la fisiología del paciente, y poder tener en cuenta la incertidumbre en sus parámetros. Este tipo de estrategia se ha utilizado tanto en la propuesta de dosificación automática en lazo abierto como en el algoritmo en lazo cerrado. Además la principal idea de diseño de esta última propuesta es evitar la sobreactuación del controlador, evitando hipoglucemias y añadiendo robustez ante fallos en el sensor de glucosa y en la estimación de las comidas. Los algoritmos propuestos han sido validados en simulación y en clínica. 


\section{Resum}

La diabetis mellitus tipus 1 és una malaltia crònica i incurable que afecta milions de persones en tot el món. Es caracteritza per una destrucció total o parcial de les cèl·lules beta del pàncrees. Aquestes cèl-lules són les encarregades de produir la insulina, hormona principal en el control de glucosa en sang. Valors alts de glucosa en la sang mantinguts en el temps afecten negativament la salut, provocant complicacions de diversa índole. És per això que els pacients amb diabetis mellitus tipus 1 necessiten rebre insulina de forma exògena.

Des que s'aconseguís en 1921 aïllar la insulina per a poder utilitzar-la en clínica humana, i es començaren a desenrotllar les primeres tècniques de monitorització de glucèmia, s'han produït grans avanços en el tractament amb insulina. Actualment, les línies d'investigació que s'estan seguint en relació a la millora de la qualitat de vida dels pacients diabètics, tenen fonamentalment 2 vessants: un primer que es centra en la investigació de cèl-lules mare per a la reposició de les cèl-lules beta i un segon vessant de caràcter més tecnològic. Dins d' aquest segon vessant, estan obertes diverses línies d'investigació, entre les que es troben el desenrotllament de nous anàlegs d'insulina que permeten emular més fidelment la secreció del pàncrees, el desenrotllament de monitors continus de glucosa no invasius i de bombes d'insulina capaces d'administrar distints perfils d'insulina i la inclusió de sistemes d'ajuda a la decisió i telemedicina. El major repte al què s'enfronten els investigadors és el d'aconseguir desenrotllar un pàncrees artificial, és a dir, desenrotllar algoritmes que permeten disposar d'un control automàtic de la glucosa.

La principal barrera que es troba per a aconseguir un control rigorós de 
la glucosa és l'alta variabilitat que presenta el seu metabolisme. Açò és especialment significatiu durant la compensació dels menjars. Aquesta variabilitat junt amb el retard en l'absorció i actuació de la insulina administrada de forma subcutània afavorix l'aparició d'hipoglucèmies tardanes (complicació aguda més important del tractament amb insulina) a conseqüència de la sobreactuació del controlador.

Les propostes presentades en aquest treball fan especial insistència en suportar aquestes dificultats. Així, s'utilitzen models intervalares per a representar la fisiologia del pacient, i poder tindre en compte la incertesa en els seus paràmetres. Aquest tipus d'estratègia s'ha utilitzat tant en la proposta de dosificació automàtica en llaç obert com en l' algoritme en llaç tancat. A més, la principal idea de disseny d'aquesta última proposta és evitar la sobreactuació del controlador, evitant hipoglucèmies i afegint robustesa davant de fallades en el sensor de glucosa i en l'estimació dels menjars. Els algoritmes proposats han sigut validats en simulació i en clínica. 


\section{Contents}

Introduction and objectives

XXIII

1 Glucose metabolism and diabetes mellitus 1

1.1 Introduction to glucose metabolism . . . . . . . . . . . 2

1.1.1 Endocrine regulation of glucose metabolism . . . . . . 2

1.2 Diabetes Mellitus: definition and history . . . . . . . . . . . . 6

$1.2 .1 \quad$ Brief history of the disease . . . . . . . . . . . 7

1.2 .2 Types of diabetes $\ldots \ldots \ldots \ldots \ldots$

1.3 DM1 and complications of the disease $\ldots \ldots \ldots . \ldots . . \ldots$

1.4 Simulating in DM1 . . . . . . . . . . . . . . . . . . . . . . . 14

1.4.1 Hovorka's group simulator [71, 177] . . . . . . . . . 16

1.4 .2 UVa Simulator [88, 100, 38, 37] . . . . . . . . . . . . 20

1.5 Conclusions $\ldots \ldots \ldots \ldots \ldots \ldots$

\begin{tabular}{|ll|}
2 & Glucose control in type 1 diabetes \\
\hline
\end{tabular}

2.1 Metabolic control monitoring . . . . . . . . . . . . . 28

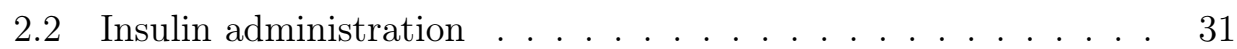

2.2 .1 Administration devices . . . . . . . . . . . . . . . . . 32

2.3 Open-loop glucose control in type 1 diabetes: intensive insulin

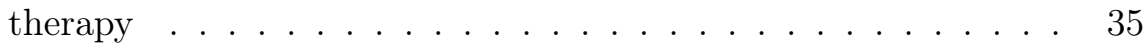

$2.3 .1 \quad$ Intensive insulin therapy: MDI and CSII . . . . . . . . 36

2.4 Closed-loop glucose control in type 1 diabetes: Artificial Pancreas 40

$2.4 .1 \quad$ Main challenges . . . . . . . . . . . . . . . . . . . . 42

2.4 .2 Artificial pancreas nowadays . . . . . . . . . . . . . . . . . . . . . . . . .

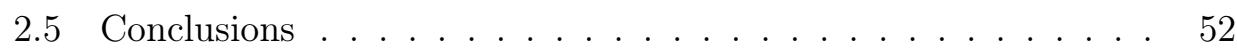


3 Open-loop strategy: Combining basal-bolus insulin infusion for tight postpandial glucose control $\quad 55$

3.1 Introduction . . . . . . . . . . . . . . 56

3.2 Theoretical framework: Interval Analisis and Set Inversion . . . 58

3.3 Set-Inversion-Based prandial insulin delivery . . . . . . . . . . . 61

3.3.1 Output of the algorithm: best basal-bolus combination . 65

3.4 In silico evaluation . . . . . . . . . . . . . . . . . . . . . . . . . 69

3.4 .1 Patient's model identification . . . . . . . . . . . 71

3.4.2 Metrics used in the in silico evaluation . . . . . . . . . . 74

3.4 .3 Results of the in silico evaluation . . . . . . . . . . . . 77

3.4 .4 Discussion of the in silico evaluation . . . . . . . . . . . 82

3.5 Clinical validation . . . . . . . . . . . . . . . . . . 86

3.5.1 Characteristics of the study . . . . . . . . . . 87

3.5.2 Metrics used and results of the clinical validation . . . . 88

3.5 .3 Discussion of the clinical validation . . . . . . . . . . 89

3.6 Conclusions $\ldots \ldots \ldots \ldots \ldots$

4 Closed-loop strategy: A reference conditioning method for $\begin{array}{ll}\text { the reduction of hypoglycemia } & 95\end{array}$

4.1 Introduction . . . . . . . . . . . . . . . . . . . . . . 96

4.2 Theoretical framework $\ldots \ldots \ldots \ldots$

4.2 .1 Invariance control . . . . . . . . . . . . . . . . . . . . . . 99

4.2 .2 Finite-time invariance achievement via Sliding Mode Reference Conditioning . . . . . . . . . . . . . 101

4.3 Safety auxiliary feedback element (SAFE) in diabetes control . 103

$4.3 .1 \quad$ Basics of the algorithm . . . . . . . . . . . . 103

4.3 .2 Robustness and fault-tolerance properties . . . . . . . 107

$4.3 .3 \quad$ SM establishment on safety $I O B$ constraints . . . . . 109

4.3 .4 IOB constraints definition . . . . . . . . . . . . . . . 109

4.4 Simulations and results . . . . . . . . . . . . . . . . . . . . . 112

4.4.1 SAFE algorithm simulations illustrating robustness . . . 112

4.4 .2 In-silico evaluation using UVA simulator . . . . . . . . 115

4.5 Conclusions . . . . . . . . . . . . . . . . . . . . . . . . . 119 
5 Extending SAFE to improve its performance against variablity 123

5.1 Theoretical framework . . . . . . . . . . . . . . . . . . 124

$5.1 .1 \quad$ Monotonicity analysis . . . . . . . . . . . . . . . 124

5.2 Interval version of SAFE $\ldots \ldots \ldots \ldots$

5.3 Simulations and results . . . . . . . . . . . . . . . . . 130

5.4 Conclusions $\ldots \ldots \ldots \ldots \ldots \ldots$

$\begin{array}{lll}6 & \text { Conclusions } & 137\end{array}$

6.1 Publications . . . . . . . . . . . . . . . . . . . . 139

$\begin{array}{ll}\text { References } & 143\end{array}$ 



\section{List of Tables}

1.1 Aetiology classification of diabetes. Adapted from [159] . . . . . 10

1.2 Chronic Complications of Diabetic Mellitus . . . . . . . . . . 12

2.1 Artificial Pancreas routes. Adapted from [22]. . . . . . . . . . 44

2.2 Current clinical studies validating closed-loop control systems. 53

3.1 Demographic, anthropometric and metabolic parameters of the 30 in silico subjects available in the educational version of the UVa simulator. . . . . . . . . . . . . . . . . 70

3.2 Proper four meals experiment setup according to the OED . . . 74

3.3 Model parameters estimation . . . . . . . . . . . . . . 76

3.4 Mean Different Indicator Values for the 10 Adults in the University of Virginia Simulator. . . . . . . . . . . . 78

3.5 Mean Different Indicator Values for the 10 Adolescents in the University of Virginia Simulator . . . . . . . . . . . . . . . . . 79

3.6 Mean Different Indicator Values for Nine Children Analyzed from Those Available in the University of Virginia Simulator. . 80

3.7 Glycemic Variability over the Course of Five and Seven Hours . 81

3.8 Demographic, anthropometric and metabolic parameters of the patients participating in the study . . . . . . . . . . 88

3.9 Insulin Dose and the Area Under the Curves of Plasma Glucose, Plasma Glucose Above $140 \mathrm{mg} / \mathrm{dl}$, and Glucose Infusion Rate of the 5-h Postprandial Period for the maximal-bolus solution and the standard therapy $\ldots \ldots \ldots . \ldots . \ldots 90$ 
3.10 Intrasubject Variability of the Postprandial Glycemic Response Reported as the Intra-Individual Coefficient of Variation of the 5-h Postprandial Period . . . . . . . . . . . . . . . . 90 90

$4.1 \quad$ PID tuning for each of the patient $\ldots \ldots \ldots \ldots$

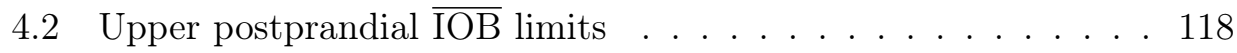

4.3 Different indicators for the evaluation of SAFE $\ldots \ldots \ldots$

5.1 Different indicators for the evaluation of interval SAFE . . . . . 134 


\section{List of Figures}

1.1 Physiologic anatomy of an islet of Langerhans in the pancreas [62]. 3

1.2 Glucose regulation in resting conditions. . . . . . . . . . . . 4

1.3 Glucose regulation in activity conditions. . . . . . . . . . . . 4

1.4 Glucose regulation after a meal. . . . . . . . . . . . . . . 5

1.5 Blood glucose curve after ingestion of $1 \mathrm{~g}$ of glucose per $\mathrm{kg}$ of

\begin{tabular}{|c|}
\hline body weight in a person with (red line) and without (green line) \\
\hline
\end{tabular}
diabetes $62 . \ldots \ldots \ldots \ldots \ldots \ldots$. . . . . . . . . . . . 7

$1.6 \quad$ Frederic Banting, canadian physician and one of the discoverers of insulin. . . . . . . . . . . . . . . 7

1.7 Main chronic complications of DM. . . . . . . . . . . . 12

1.8 Diabetic body physiology. . . . . . . . . . . . . . . 15

1.9 Hovorka's model implemented in the simulator. . . . . . . . . 18

1.10 Cobelli's insulin model. . . . . . . . . . . . . . . . . . . 21

1.11 Dalla Man's gastrointestinal model. . . . . . . . . . . . . . . 22

1.12 Cobelli's glucorregulatory model. . . . . . . . . . . . . . 23

2.1 Molecular structure of human insulin. . . . . . . . . . . . . . 31

2.2 Different insulin effects. Adapted from [43]. . . . . . . . . . . 32

2.3 External insulin administration devices. . . . . . . . . . . . 35

2.4 Different intensive insulin therapy regimens. Adapted from [81]. The $\mathrm{x}$-axis shows the time of the day: B, breakfast; L, lunch and D, dinner. Vertical arrows indicate the time of insulin injection/ bolus infusion. . . . . . . . . . . . . . . . . 37

2.5 Graphical representation of different bolus waveforms implemented in insulin pumps. . . . . . . . . . . . . . . . . . . . . . 40 
$2.6 \quad$ First insulin pump developed by Kadish in 1964. . . . . . . . . 41

$2.7 \quad$ Schematic representation of a glucose closed-loop system using

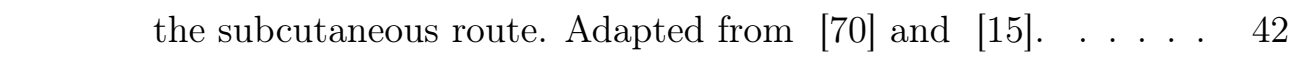

2.8 Delays in insulin action depending on the route used. Adapted \begin{tabular}{|c|}
\hline from [68]. Last transport lag is only present in microdialisys or \\
\hline
\end{tabular} microperfusion-based systems. . . . . . . . . . . 43

2.9 Response of each PID part to a hyperglycemic clamp. P \begin{tabular}{|c|}
\hline component is proportional to the error, I component increases if \\
\hline the time outside the target increases and D component is active \\
\hline
\end{tabular}

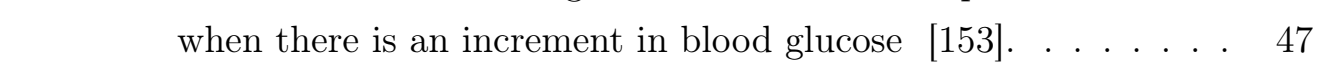

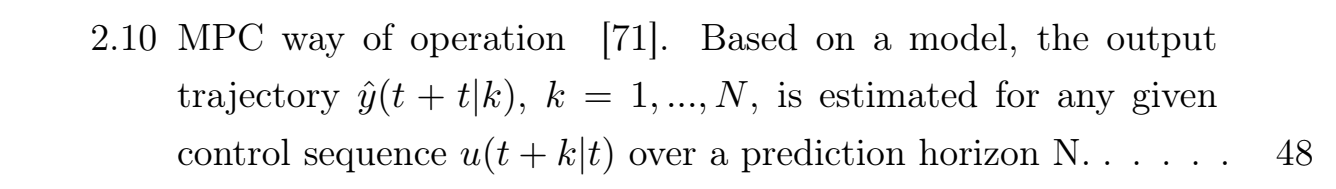

$3.1 \quad$ Plot that illustrates the concept of inner and outer subpaving. \begin{tabular}{|l|}
\hline Blue rectangles represent the inner subpaving and guarantee the \\
\hline fulfillment of the constraints. The outer subpaving is made up of \\
\hline both blue and red rectangles. Its complementary set (in white) \\
\hline is guaranteed to contain only non-solutions that violate some \\
\hline of the constraints. Results in the boundary (red rectangles) are \\
\hline
\end{tabular} - unknown a priori. . . . . . . . . . . . . . . . 60

$3.2 \quad$ Output of an interval simulation. Upper and lower envelopes

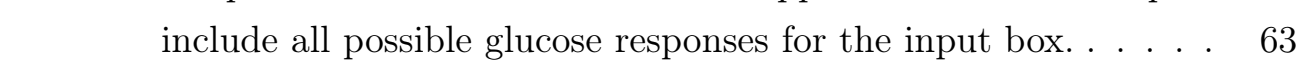

3.3 Input and outputs of the designed algorithm. By using a

\begin{tabular}{|c|}
\hline mathematical model of the patient and information regarding \\
\hline the meal to be ingested, the SIVIA based algorithm calculates \\
\hline the best basal-bolus insulin dose combination to fulfill a set of \\
\hline
\end{tabular}

\begin{tabular}{|l|}
\hline the best basal-bolus insulin dose combination to fulfill a set of \\
\hline constraints according to the IDF criteria of good postprandial \\
\hline
\end{tabular}

3.4 Plot that represents a 3D (basal, bolus and time) feasible set

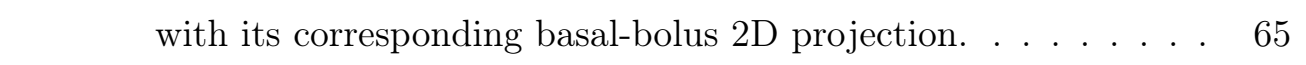


3.5 Normalized feasible set that shows all the possible bolus administration modes. Therapies with nominal basal correspond to a standard strategy, therapies with an increment in basal postprandial dosage result in a dual-wave or square-wave strategy whereas therapies with less postprandial basal than baseline are called here as temporal basal decrement mode. The corresponding insulin infusion profiles are depicted for each region. 66

$3.6 \quad 2 \mathrm{D}$ basal-bolus projection of normalized feasible sets for a meal of 40 to $140 \mathrm{~g}$ of carbohydrates and initial normoglycemia. The \begin{tabular}{|c|}
\hline vertical line stands for the standard strategy with basal equal \\
\hline
\end{tabular} to its baseline value. . . . . . . . . . . . . . . . . 67

3.7 Procedure used to select the basal-bolus combination from all the available possibilities. . . . . . . . . . . . . . . . . . . . 69

3.8 Value of the D-optimality criterion against different number of meals for the three groups of patients. . . . . . . . . . . 73

3.9 Four-meal identification following the OED results. The solid red line represents the glucose profile of the virtual patient whereas the dotted blue line represents the profile simulated by the identified model. . . . . . . . . . . . . . . 75

3.10 Mean glucose response of the 10 adults in the UVa simulator. The blue line represents the response applying the standard bolus, whereas the green and the red line correspond to the centroid and maximal-bolus solution, respectively. These latter solutions produce a flatter profile than the one observed with the standard bolus, avoiding late hypoglycemia. In addition, the peak in the glucose profile remains similar or even lower. . 82 
3.11 Mean glucose response of the 10 adolescents in the UVa simulator. The blue line represents the response applying the standard bolus, whereas the green and the red line correspond to the centroid and maximal-bolus solution, respectively. These latter solutions produce a flatter glucose profile than the one observed with the standard bolus, avoiding late hypoglycemia. The peak in the glucose profile using any of the solutions is similar

3.12 Mean glucose response of nine children analyzed from those available in the UVa simulator. The blue line represents the response applying the standard bolus, whereas the green and the red line correspond to the centroid and maximal-bolus solution, respectively. These latter solutions, although producing a slightly higher peak in the glucose profile than the standard bolus, achieve a flatter glucose profile, avoiding the severe late hypoglycemia.

4.1 Invariance condition. Adapted from [128]. . . . . . . . . . 100

4.2 Generic scheme of an SMRC loop. . . . . . . . . . . . . 102

$4.3 \quad$ SMRC implementation for diabetes application. . . . . . . . . . 104

4.4 Schematic representation and equations of Cobelli's insulin model used here to estimate IOB. . . . . . . . . . . . . . . 105

4.5 Daily IOB constraint function. . . . . . . . . . . . . . 111

4.6 Comparison of the performance of the controller in one virtual patient (patient 4) with a conventional PID and with a PID plus the additional SAFE loop during one day. The glucose profile, together with the IOB profile, and the discontinuous signal with the realizable reference derived from it are presented. Additionally, the actual response of the controller with and without SAFE is also shown. . . . . . . . . . . . . . . . 113 
4.7 Glucose response,IOB profile and controller response (no priming bolus is considered here) in the presence of sensor failures. Blue lines represent the response without SAFE loop whereas red lines correspond to the response using SAFE. In the glucose profiles, solid lines are the sensor signal and dotted lines the actual glucose profile. . . . . . . . . . . . . . . . . . . . 114

4.8 Plot that illustrates the SAFE reaction against over-estimated

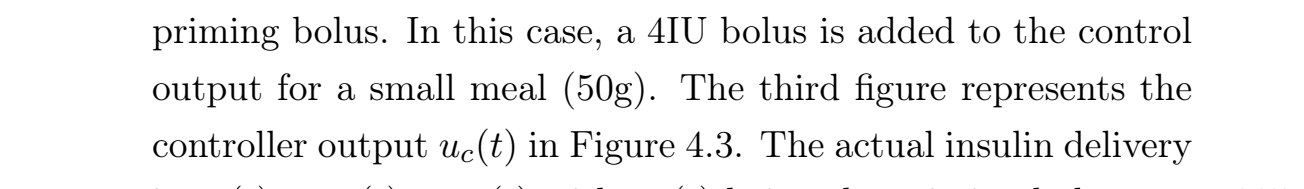
is $u_{p}(t)=u_{c}(t)+u_{f}(t)$ with $u_{f}(t)$ being the priming bolus. . . 115

4.9 Plot that illustrates the SAFE reaction against over-estimated priming bolus in case $w^{+}$has not been designed high enough. The IOB limit is temporally violated but SAFE always contributes to enter again in the allowed region with $w(t)=w^{+} . \quad$. . 116

4.10 Plot that shows the AUC of $P G<70 \mathrm{mg} / \mathrm{dl}$ for the 10 available virtual bolus for a $50 \mathrm{~g}$ meal and a feed-forward bolus $30 \%$ higher than the usual. . . . . . . . . . . . . . . . . 116

4.11 Percentage of time in hypo and hyperglycemia of each patient with and without SAFE loop. . . . . . . . . . . . . . 120

5.1 Example of a monotone (A) and a non-monotone system (B) [41].125

5.2 Parameters monotonicity of systems $5.1 \mid$ and $\mid 5.3$. . . . . . . 127

5.3 Output bounds for the IOB in Cobelli's insulin absorption model developed. $S_{1}(0)=183, S_{2}(0)=194$ and $k_{a 1}=0.0034$, $k_{a 2}=0.0159, k_{d}=0.0161$ under $60 \%$ uncertainty (worst case in the Cobelli's adult population). . . . . . . . . . . . 128

5.4 Comparison of the performance of the controller in one virtual patient (patient 9) for the 3 options: PID, PID+SAFE and PID + SAFE intervalar loop during one day. Glucose profile and IOB curve (both the IOB estimated and the real IOB profile) are shown. . . . . . . . . . . . . . . . . . 131 
5.5 Plasma glucose profile, IOB estimation together with the discontinuous signal and weighting coefficient that resulted from the fuzzy system for the PID + the interval version of SAFE. The dotted lines in the IOB curve represent the outer and the inner bounds of the IOB estimation. Note that according to the weighting coefficient the IOB curve used in the algorithm is closer to one or the other bound. . . . . . . . . . . . . . . . . 132

5.6 Total percentage of time in hypoglycemia $P G<70$ of each patient with the three strategies . . . . . . . . . . . . . . . 134

5.7 Percentage of time in hypo and hyperglycemia of each patient with standard PID, with the additional SAFE loop and with the version of SAFE using interval IOB. . . . . . . . . . . . 135 


\section{Introduction and objectives}

Diabetes mellitus (DM) comprises a group of common metabolic disorders that share the phenotype of high glucose levels in blood (hyperglycemia). Causes for this hyperglycemia are different, from an insufficient insulin production by the pancreas, type 1 diabetes mellitus (DM1), to an insulin resistance, type 2 diabetes mellitus (DM2).

According to data from the International Diabetes Association [77], diabetes currently affects more that 360 million people around the world (about $6 \%$ of the total world population). Predictions estimate that these figures are still growing and by the year 2030 the number of diabetic people will reach 552 million.

These patients are at risk of developing different kinds of long-term complications (ophthalmologic complications, renal disorders, cardiovascular pathologies...) as a consequence of the disease. In fact, in the United States, DM is the leading cause of end-stage renal disease, non traumatic lower extremity amputation, and adult blindness [81].

About $10 \%$ of diabetic patients suffer from DM1 and they depend on exogenous insulin for survival. Several studies, such as the Diabetes Care and Complications trial (DCCT) [61] and the Epidemiology of Diabetes Interventions and Complications (EDIC) [109] have demonstrated that any improvement in the metabolic control of diabetic patients, helps in reducing those complications. In order to remain as much as possible within safety glucose values, patients have to follow an intensive insulin therapy that tries 
to reproduce the insulin secretion profile of a healthy person. This therapy can be administered using multiple daily insulin injections (MDI) or a continuous subcutaneous insulin infusion (CSII) using insulin pumps. From a control point of view, two control actions can be considered. On one hand, the insulin needed to keep the glucose concentration in safety values (around $100 \mathrm{mg} / \mathrm{dl}$ ) during the night and between meals (basal insulin); and, on the other hand, the insulin needed to counteract the effect of the meals (bolus insulin).

One of the biggest concerns related to glucose control in DM1 is meal counteraction. Modern insulin pumps are capable of administering short-time insulin continuously, adding more flexibility and allowing different profiles in the postprandial time (after the meals) [32]. They also include tools for helping in the postprandial bolus decision-making process (bolus-advisors) [184. However, despite the development of these new tools, postprandial control optimization is still an empirical process based both in physicians' and patients' experience.

Moreover, meals are also the greatest perturbation complicating the development of the so-called "artificial pancreas", final objective of the research in this area. Recent development of continuous glucose monitoring systems (CGMS) together with the already mentioned insulin pumps, has fostered an increase in the efforts for achieving automatic glucose control systems (the artificial pancreas). Controllers which are already being validated in clinical practice, mainly based on proportional-integral-derivative controllers (PIDs) and model predictive control (MPC) [68] [48], are finding in the increase in hypoglycemia event: $\mathrm{1}^{1}$ during the late postprandial period the main barrier complicating the control [24]. These hypoglycemias are mainly caused by an overcorrection to the meal perturbation due to a delay in subcutaneous insulin absorption.

An additional problem of the metabolic control in DM1 is the high variability found in the physiologic behavior. This variability exists not only between different patients but also in a same patient in different times or

\footnotetext{
${ }^{1}$ Too low glucose concentrations (bellow $70 \mathrm{mg} / \mathrm{dl}$ ) that are potentially dangerous for the patient, especially if they are kept too long.
} 
situations. This variability and uncertainty has to be taken into account when designing both open-loop and closed-loop control strategies.

Indeed meal counteraction avoiding late hypoglycemia is still an unsolved problem both in open and closed-loop strategies, mainly due to the delays in subcutaneous insulin action and to the high inter and intra-patient variability.

In this context, the main objective of this thesis work is:

"To provide the scientific community with strategies that add robustness to the glucose control of type 1 diabetic patients, especially in the postprandial period."

The search of this objective has lead to a set of results:

- Review of the characteristics of DM disease and its impact in the patients and in the world-wide health system.

- Review of the therapies that are currently applied to counteract the effects of DM, the main challenges that still remain unsolved and the lines to improve the performance of the glucose control algorithms already present in literature.

- An strategy to improve open-loop glucose control in the postprandial period.

- An strategy to improve closed-loop glucose control adding robustness against uncertainty in the parameters and in the measures.

These results are presented across the different chapters. Chapter 1 and Chapter 2 review the main characteristics of Diabetes Mellitus. The attention is focused on the different models used to represent the metabolism involved in the disease and in the challenges that current therapies need to overcome.

In Chapter 3 an algorithm based on set inversion via interval analysis is used to coordinate basal and bolus insulin infusions to deal with postprandial glucose excursions. Chapter 4 and Chapter 5 present an algorithm to be added to any glucose closed-loop controller to provide an additional safety layer 
against hypoglycemias. The algorithm, called SAFE and based on sliding mode reference conditioning techniques (SMRC), adds constraints in the residual insulin activity being robust against uncertainty in the patient metabolism behavior.

A set of conclusions and summary of the results can be found in Chapter 6 


\section{Glucose metabolism and dia- betes mellitus}

Glucose is the main source of energy for the cells. In humans, a complex metabolic regulation of glucose, where several hormones are involved, balances the glucose storage and production (basically in the liver); and, the utilization from mainly the muscle, the adipose tissue and the brain. Due to this regulation, plasma glucose is normally kept within a narrow range (approximately $65-140 \mathrm{mg} / \mathrm{dl})$.

However, in people with DM the regulation of glucose metabolism is altered and their body is not capable of maintaining blood glucose in that narrow range naturally. Excessive increase in plasma glucose concentration over years is potentially dangerous for diabetic patients whose rate of cardiovascular morbidity and mortality is twice the general population. Moreover, maintenance of plasma glucose above the threshold of hypoglycemia is critical for survival of the whole body.

In this chapter, an introduction of the main actors participating in the regulation of glucose metabolism is presented (Section 1.1). Then, Sections 1.2 and 1.3 deal with a general review of DM, focusing on DM1 which is the one of interest in this thesis. 


\subsection{Introduction to glucose metabolism}

Metabolism is defined as the energy management for maintaining the living state of the cells and the organism. Nutrition is the key to metabolism. The pathways of metabolism rely upon nutrients that they breakdown in order to produce energy. These nutrients are provided through the diet and can be classified as: carbohydrates, proteins, fat, minerals, vitamins and water.

Carbohydrates are one of the main types of nutrients. They are the most important source of energy for our body. During the digestion they are divided into monosaccharides (mainly glucose) that are absorbed through the small intestine walls into the bloodstream to be used by body cells. The way a healthy body regulates glucose metabolism is explained in this section.

\subsubsection{Endocrine regulation of glucose metabolism}

An important part of the glucose absorbed in the intestine, is stored in the liver (with the help of the insulin) in the form of glycogen. This glycogen can be transformed again into glucose in case it is needed (during the night or between meals, when there is not exogenous contribution of glucose). The glucose not stored in the liver is distributed through the circulatory system to the rest of the body cells. Muscles are also capable of storing glucose in form of glycogen, but, unlike what happens in the liver, the glucose of the muscles can only be consumed by physical exercise.

Several hormones are needed in order to regulate this glucose metabolism. The most important ones are insulin and glucagon.

The main function of insulin is to promote the absorption of glucose by the cells and to promote the glycogen synthesis in the liver (glycogenesis), diminishing blood glucose concentration. Glucagon, on the other hand, increases blood glucose concentration when it is needed, favoring the glycogen degradation to obtain glucose (glycogenolysis). Both hormones are produced by special cells present in the endocrine part of the pancreas, grouped in clusters called "Langerhans islets". 
Langerhans islets contain $\beta$-cells (producing insulin), $\alpha$-cells (producing glucagon), $\delta$-cells (producing somatostatin) and PP-cells (producing pancreatic polypeptide). $\beta$-cells are the most numerous (around $60 \%$ of the cells in the islets) and are located mainly in the islets' heart, whereas $\alpha$ and $\delta$-cells are located in their periphery. Islets cells interact with each other through direct contact and through their products. In that way, for example, insulin inhibits the production of glucagon and, somatostatin inhibits the production of insulin and glucagon [62. In Figure 1.1 the distribution of these cells in the Langerhans Islets is shown.

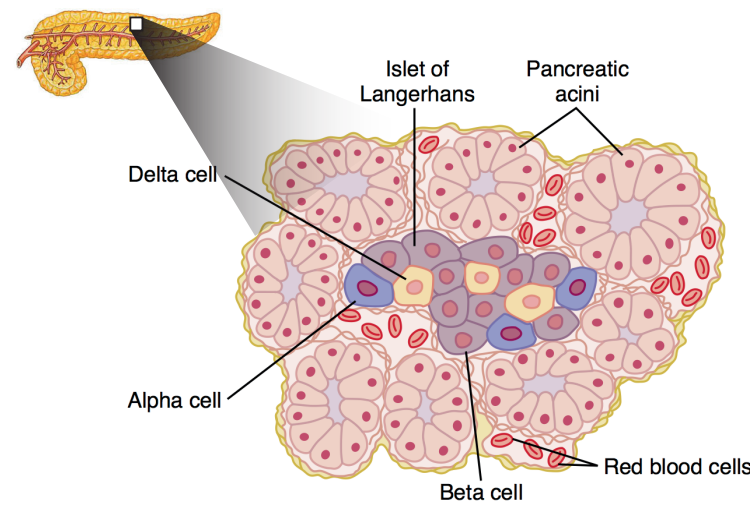

Figure 1.1: Physiologic anatomy of an islet of Langerhans in the pancreas [62].

A brief description of the main mechanisms of metabolism regulation in different situations is provided bellow. In this regulation, insulin, glucagon and the different organs and tissues are involved.

\section{In resting conditions}

In resting conditions, the glucose in blood is almost constant, although the organs keep consuming it. The glucose used is replaced either by the liver from the stored glycogen (glycogenolysis) or by the generation of glucose from fats and proteins (gluconeogenesis). Therefore, to keep the level constant a combined action between glucagon (to transform glycogen into glucose) and insulin (to help the distribution of the glucose to the organs through blood) is needed (Figure 1.2. 


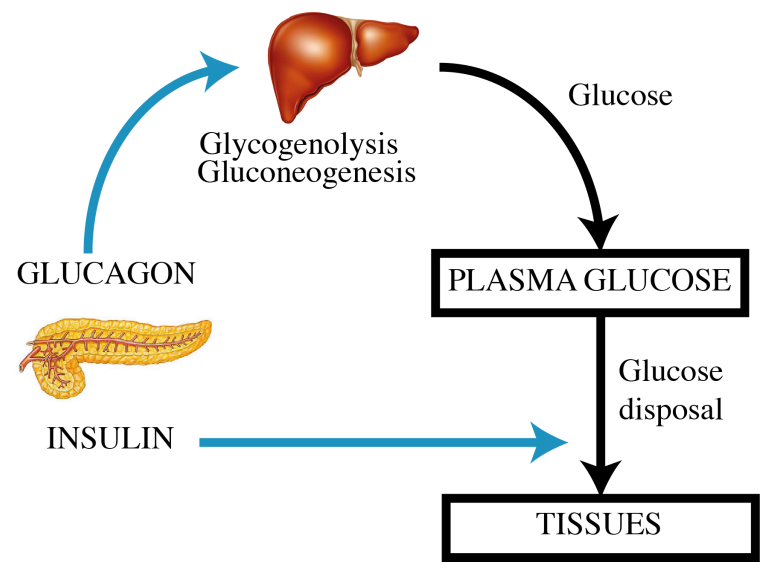

Figure 1.2: Glucose regulation in resting conditions.

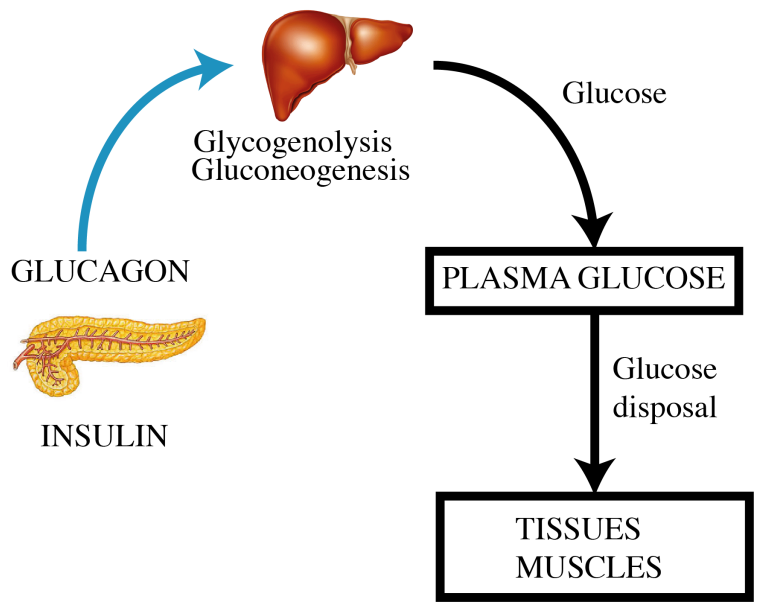

Figure 1.3: Glucose regulation in activity conditions. 


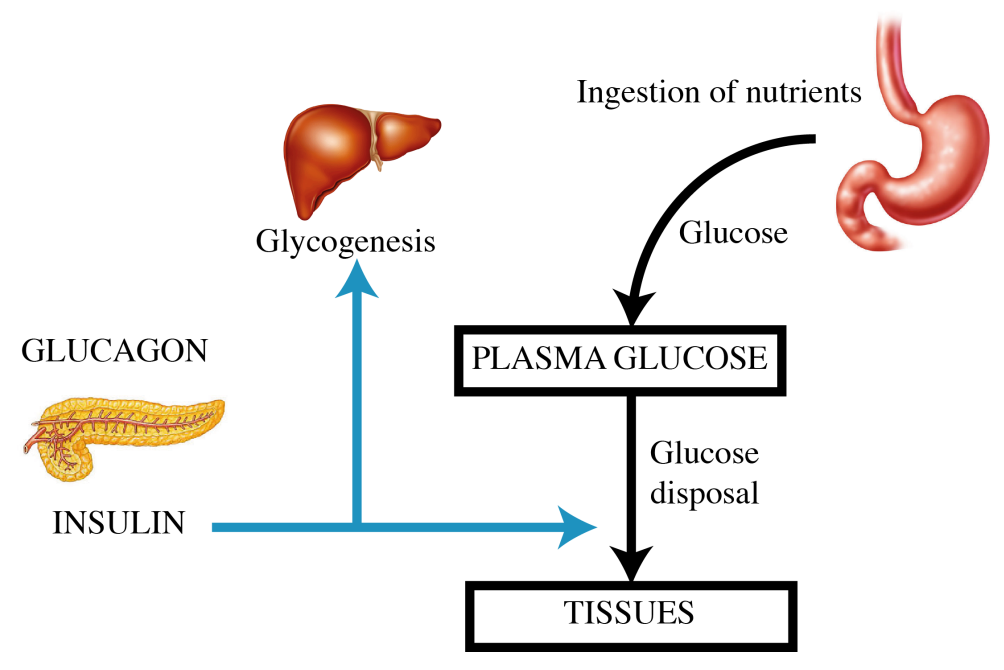

Figure 1.4: Glucose regulation after a meal.

\section{In activity conditions}

In activity conditions, the demand of glucose by the muscles increases considerably. In this situation, the secretion of insulin is reduced and the glucagon secretion increased, in order to favor the degradation of glycogen into glucose in the liver (glycogenolysis). In these conditions of physical exercise the muscles do not need insulin to absorb the glucose. Thus, the glucose obtained via the glycogenolysis can be directly used by them (Figure 1.3).

If the activity lasts a long period of time, the glucose can reach a very low level, even though it is regulated. This is due to the fact that when there are no more reserves of glycogen in the liver, the only source of glucose is the synthesis from amino acids and non-carbohydrate carbon substrates (gluconeogenesis). The amount of glucose obtained by this method is not enough for covering the demand of the muscles during high activity conditions.

\section{After a meal}

After eating a meal rich in carbohydrates, the $\beta$-cells receive signals to 
increase the synthesis and secretion of insulin. In the same way, the secretion of glucagon is reduced.

The insulin goes through the blood to the different cells, favoring the glucose transport into the cell. Most of the glucose is stored in the liver in the form of glycogen. The amount exceeding the space of the liver is transformed into fats. Once the level of glucose is stable, the $\beta$-cells stop the extra secretion of postprandial insulin (Figure 1.4).

\subsection{Diabetes Mellitus: definition and history}

The term diabetes was first used in 2nd century $\mathrm{BC}$ in greek medicine. Its etymological meaning is "passer-through, siphon" and it was used in reference to a disease characterized by the elimination of huge quantities of urine.

DM, or simply, diabetes, can be defined as a group of diseases characterized by high blood glucose levels (hyperglycemias) that result from defects in the body's ability to produce and/or use insulin. In a healthy person, the blood glucose concentration is narrowly controlled, usually between 80 and $90 \mathrm{mg} / \mathrm{dl}$ in the fasting period, each morning before breakfast. This concentration increases to 120 to $140 \mathrm{mg} / \mathrm{dl}$ during the first hour or so after a meal, but the feedback system for control of blood glucose returns the glucose concentration rapidly back to the control level, usually within 2 hours after the last absorption of carbohydrates. Conversely, in starvation, the liver provides the glucose that is required to maintain the fasting glucose level. In diabetic patients, both fasting blood glucose concentration and the glucose rise after the ingestion are abnormally increased. Moreover, they present a slower fall of the glucose concentration curve. These differences in the blood glucose curve are shown in Figure 1.5 where the glucose concentration curves of a person with and without diabetes after ingesting $1 \mathrm{~g}$ of glucose per $\mathrm{kg}$ of body weight are plotted.

The main symptoms of a continuous hyperglycemia are polyuria (frequent urination), polydipsia (increased thirst and fluid intake) and polyphagia (increased appetite). A chronic hyperglycemia can cause damage, disfunction 


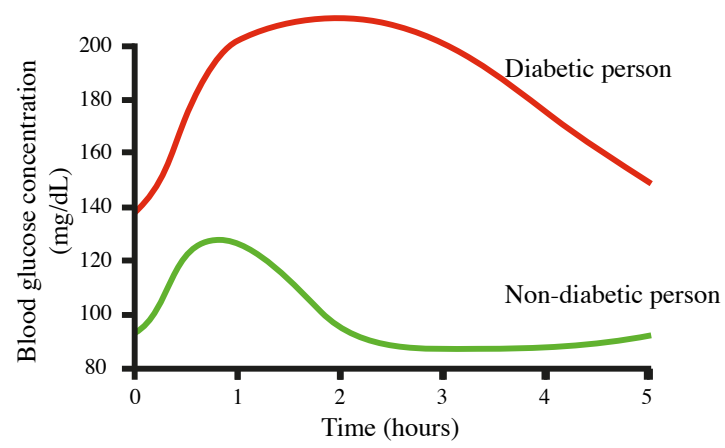

Figure 1.5: Blood glucose curve after ingestion of $1 \mathrm{~g}$ of glucose per $\mathrm{kg}$ of body weight in a person with (red line) and without (green line) diabetes [62].

or failure in some organs such as the eyes, the kidneys, nerves, heart and blood vessels.

\subsubsection{Brief history of the disease}

As it occurs with many other diseases, the main advancements in the study and knowledge of DM have been carried out during the 20th century. In fact, it is not until 1922 when insulin begins being used for human clinical practice. This advance is achieved thanks to Grant Banting (1891-1941) and Charles Best (1899-1978) who, in 1921, succeeded in isolating insulin [17. Because of this deed, Banting was laureated with the nobel prize in 1923.

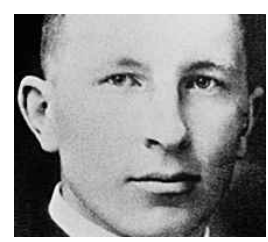

Figure 1.6: Frederic Banting, canadian physician and one of the discoverers of insulin.

Despite the fact that the biggest advances in the field took place during the last century, the first reference to the disease dates back to 1550 B.C. in 
the popular Ebers Papyrus? In that papyrus a symptomatology similar to diabetes is described. Diabetes ("a passer-through, siphon" in greek) owes its name to Areteo of Capadocia who named it that way in reference to an excess of urine. From the 10th to 11th century A.D., it was in Arabic medicine where most progress was made, mainly because of the works of Avicenna (980-1037) and Averroes (1126-1198) [106.

We have to wait until 17 th century to find great advances in the occidental world. In that way, Thomas Willis (1621-1675) in 1674 pointed out the sweet taste in the urine of diabetic people, which gave diabetes the sub-name of "mellitus" (honey sweet). However, it was only in 1776 when Mathew Dobson (1745-1784) chemically confirmed the presence of sugar in diabetics' urine. In 1859, Claude Bernad (1813-1878) determined the presence of glucose in the blood of diabetes people. The 19th century was decisive for advancing in the physiologic understanding of diabetes. Oskar Minkowski (1858-1931) and Josef von Mering (1849-1908) proved in dogs that the absence of the pancreas caused an increment of blood glucose. This demonstration, together with the discovery of the "islets of Langerhans" in 1893 by Edouard Laguesse (18611927) 2 , fostered the interest in isolating the active ingredient of the islets of Langerhans capable of reducing blood glucose concentrations. However, it was necessary to wait until 1921 for Frederic Grant Banting (1891-1941) and Charles Best (1899-1978) to isolate a toxicity free extract of the pancreas, to be used as diabetes treatment: Insulin [39].

Nowadays, the research lines to improve the quality of life of diabetic patients are mainly two: the first one is focused on the research of stem cells to replace the damaged $\beta$-cells of the pancreas (the cells that produce the insulin). The second line has a more technical character and includes, the production of new types of insulins which are more efficient and can emulate better pancreatic secretion, the development and improvement of devices to

\footnotetext{
${ }^{1}$ The Ebers Papyrus is one of the oldest medicine treatise known. It was found in 1862 with the remains of a mommy in Luxur. After that, it was bought and translated by George Ebers. Nowadays, it is kept in the university library of Leipzig.

${ }^{2}$ The islets of Langerhans were previously described by Paul Langerhans (1847-1888) in his $\mathrm{PhD}$ thesis (1869) but it was Edouard Laguesse who suggested their implications in the endocrine part of the pancreas.
} 
measure the blood glucose and administrate the insulin and the development of support systems and telemedicine platforms. Finally, the biggest challenge in this research line is the development of the so-called "Artificial Pancreas", that is, algorithms to enable the automatic control of glucose.

\subsubsection{Types of diabetes}

The last classification of DM dates from 1997, when an expert committee of the American Diabetes Association (ADA) and of the World Health Organization (WHO) agreed on a new diabetes classification replacing the last one developed by the National Diabetes Data Group (NDDG) in 1979.

The main difference between both classifications is that the new one is based on the pathogenic process that leads to hyperglycemia, as opposed to the earliest one which used age and therapy criteria. In that way, the 1997 classification distinguishes between two broad categories, DM1 and DM2.

DM1 embraces all the kinds of diabetes where there is destruction of pancreatic $\beta$-cells and propensity to ketoacidosis? This kind of diabetes can be due to an autoimmune process or presents an unknown aetiology. The treatment for people with DM1 is the necessarily replacement of the endogenous insulin secretion by means of the administration of exogenous insulin.

DM2 is the most common type of diabetes and it is characterized by variable degrees of insulin resistance, impaired insulin secretion, and increased glucose production. It is related to various clinical risk factors such as increasing age, obesity, and racial and geographical characteristics. In most cases, this kind of diabetes can be effectively treated, at least in the early stages, with exercise, caloric restriction and drugs that increase insulin sensitivity. However, in the later stages, insulin administration is usually required to control plasma glucose 62 .

\footnotetext{
${ }^{3} \mathrm{An}$ acute complication of diabetes due to the accumulation of ketones (sub-product of the fat metabolism) when glucose is not available as organism energy source.
} 
I. Type 1 diabetes
A. Inmune-mediated
B. Unknown Aetiology

II. Type 2 diabetes

III. Other specific types of diabetes

IV. Gestational diabetes

Table 1.1: Aetiology classification of diabetes. Adapted from [159]

Other kind of diabetes also considered in 1997 classification are those induced by genetic defects in insulin secretion or action, metabolic abnormalities that impair insulin secretion, mithocondrial abnormalities and a host of conditions that impair glucose tolerance. Finally, this classification considers also gestational DM which is usually reverted after pregnancy [159]. Table 1.2.2 summarizes this classification.

This thesis is focused on glucose control in DM1 where insulin is needed as a control action from the early stages of the disease.

\subsection{DM1 and complications of the disease}

In DM1 the pancreatic $\beta$-cells are destroyed. This fact implies an absolute insulin deficit and the need of exogenous insulin for survival. The usually onset of this kind of diabetes occurs in people under 15 years (this is why it is also known as juvenile diabetes), but it can start at any age. 90\% of DM1 cases have an autoinmmune aetiology whereas the rest are idiopathic (unknown aetiology). The destruction rate of $\beta$-cells in DM1 is variable. It takes place very quickly in some patients (mainly children and teenagers) and slower in others.

The most common acute complications in DM1 are ketoacidosis and hypoglycemia.

- Diabetic ketoacidosis (also known as diabetic coma) is a state of severe and uncontrolled diabetes, caused by an insulin deficiency and it requires 
an emergency treatment with intravenous insulin and fluids. Its cause is the accumulation of sub-products coming from the metabolism of fats known as ketones. This fact happens when there is no available glucose to be used as energy source by the organism and lipids are used instead. Diabetic ketoacidosis is a serious problem with an estimated mortality between $5 \%$ and $10 \%$ in occidental countries and particularly dangerous in elderly people. Usually, it can be found in young DM1 patients (in fact in many cases it is the first symptom leading to the diagnosis of the disease) although it can be found also in patients with DM2 during severe infections or other illnesses.

- Hypoglycemia occurs as a secondary effect of the treatment with insulin. Correct function of the brain depends on the continuos supply of glucose. The interruption of the supply during more than a few minutes can cause a disfunction on the central nervous system, loss of consciousness and even coma.

Hypoglycemia in diabetics is due to an unusual increase of blood insulin concentrations or an increase in its effect. The concrete causes of hypoglycemia are diverse: an excess in the insulin dose, an increase in the bioavailability (for example, an acceleration in the absorption due to exercise), an insulin sensitivity $\left.\right|^{5}$ increment, or an inadequate carbohydrate ingestion. Other risk factors are stomach paresthesias, and alcohol or other drug consumption.

However, the major cause of mortality in diabetic patients are chronic long-term complications derived from the disease. These complications can affect several organic systems and can be divided into micro-vascular, macrovascular and other kind of complications. The risk of developing these complications increases with exposure to hyperglycemia. The most common chronic complications are briefly described [81, 63, 179]:

\footnotetext{
${ }^{4}$ The degree and velocity to which a drug or other substance becomes available to the target tissue after administration.

${ }^{5}$ Insulin sensitivity describes how sensitive the body is to the effects of insulin.
} 
- Microvascular

- Eye disease

- Neuropathy

- Nephropathy

- Macro-vascular

- Coronary artery disease

- Peripheral vascular disease

- Cerebrovascular disease

- Other

- Gastrointestinal

- Genitiurinary

- Dermatologic

- Infectious

- Cataracts

- Glaucoma

Table 1.2: Chronic Complications of Diabetic Mellitus

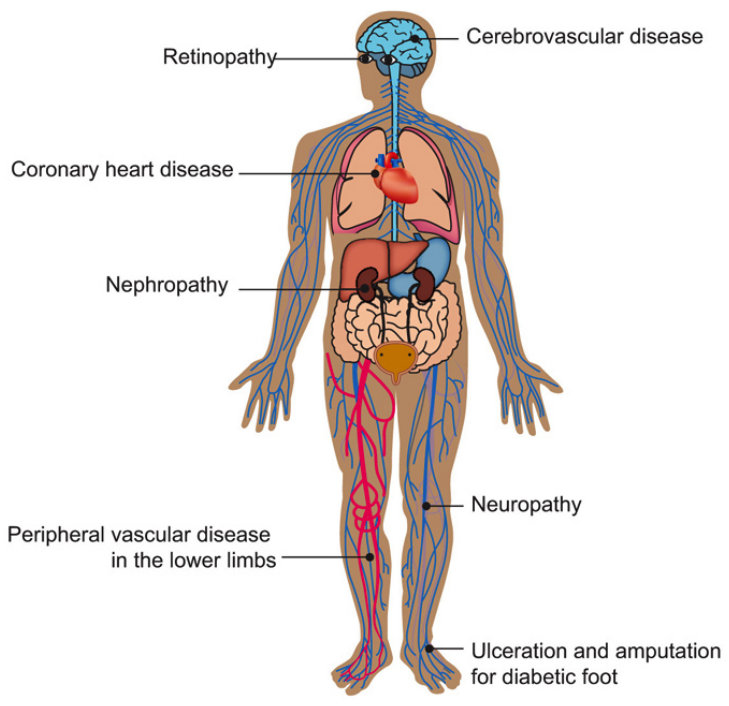

Figure 1.7: Main chronic complications of DM. 
1. Ophthalmologic complications: Diabetic retinopathy, damage in the blood vessels of the retina, is the most common diabetic eye disease and a leading cause of blindness in adults. Other ophthalmologic complications are cataracts and glaucomas. An estimated $97 \%$ of insulin-taking and $80 \%$ of non insulin-taking people who have had diabetes for more than 15 years have retinopathy; approximately $40 \%$ of insulin-taking and $5 \%$ of non insulin-taking people have the most severe stage, proliferative diabetic retinopathy.

2. Renal complications: Diabetes is the most common cause of kidney failure in developed countries. The main characteristic of this complication is the presence of protein in urine due to a disfunction in kidney blood vessels. This fact affects the operation of the kidney and can lead to chronic kidney disease and kidney failure.

3. Neuropathy: Neuropathy is a very common complication in DM. The nervous system is damaged because of high blood glucose levels that diminish blood supply. The most common neuropathy associated with diabetes affects the lower limbs, mainly the feet. Some times it can be asymptomatic, causing damage in the feet due to a loss of sensitivity.

4. Gastrointestinal disfuntion: The most common gastrointestinal problem in diabetic people is gastroparesis. Food remains in the stomach for a longer time than normal. This fact favors the overgrowth of bacterias, and causes the formation of solid masses of undigested food (beozars) that can, eventually, provoke intestinal obstruction. The cause of this problem is the damage of the vague nerve, which is in charge of controlling the digestion, due to continuous hyperglycemia.

5. Cardiovascular problems: Cardiovascular disease is increased by 2-4 times its normal rate in people with both DM1 or DM2. High blood glucose levels, together with cholesterol and high blood pressure are risk factors for artheroescloris (artery wall thickening that causes its narrowing) which is the seed of many heart and large blood vessel 
problems: coronary artery disease, cerebrovascular accidents, peripheral vascular diseases...

6. Other: Other kind of complications in DM are lower limb complications, infections and several minor dermatologic complications.

\subsection{Simulating in DM1}

As it has been reviewed through this chapter, diabetes is a complex disease with many social implications and which affects the quality of life of the patient. Models capable of reproducing the different processes that are involved in the metabolism of diabetic people can be used to help the patient to get an understanding of his disease, and how his body reacts to exercise, stress, insulin, etc... Additionally, these models are very useful for developing decision-support tools and in the process of designing and testing closed-loop algorithms for glucose control.

Depending on the modeling approach, these models can be data-driven models or physiological models. Data-driven models are focused on data reproduction, and not at all in the physiology behind it, while physiological models only use the data to adjust the parameters, while the structure is determined by the model itself. Most of the models used in diabetes are physiological models, although there are some methods that use simplification and linearization of those models to use them for control design purposes.

So as to model the physiology of the body of a diabetic patient, the main parts of the organisms that take part, in one or another way, in the glucose regulation and may be affected by the disease, must be taken into account. Many factors, difficult to model, such as stress, exercise, the presence of infections... are present in this regulation. However, the main systems that basically govern the behavior of a diabetic patient are represented by:

- Insulin absorption model

- Glucose absorption model 
- Glucorregulatory model

Figure 1.8 shows the block diagram of these three systems, where the outputs of the two former act as the inputs of the glucorregulatory system.

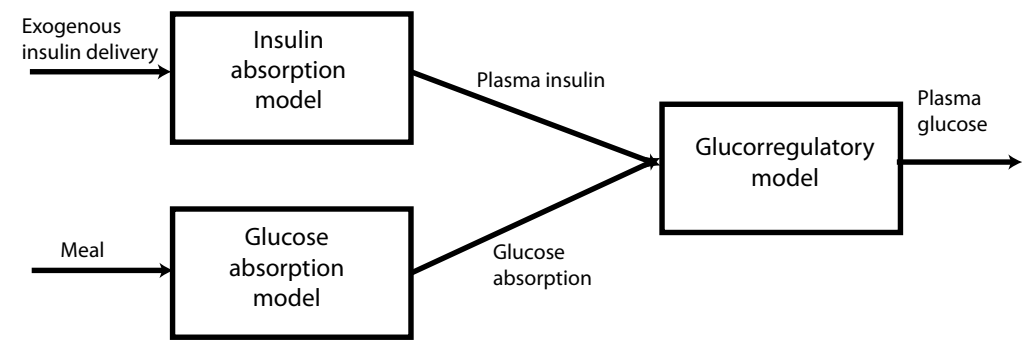

Figure 1.8: Diabetic body physiology.

The insulin absorption model represents the way insulin gets into the organism, which involves its pharmacokinetics, diffusion through different tissues and natural insulin degradation. Insulin is injected or infused in the subcutaneous tissue, delaying its appearance in plasma compared to insulin secretion by the pancreas. The glucose absorption model involves the process of ingestion, digestion and absorption from the intestine into blood of glucose and other nutrients; and, the glucorregulatory model tries to reproduce the glucose regulation explained in Section 1.1. It defines how insulin and glucose interact and determine the level of glucose in blood at the defined conditions.

During the last years many mathematical models have been developed with different purposes, as educational models [96], models for estimating insulin sensitivity [30, and models for developing new drugs [183]. But, lately, a lot of effort is concentrating in the the development of models to test new algorithms and strategies, in virtual patients (simulation). The most widely used models, both for control and for validation, and those which have already been implemented in simulation environments, are the one developed by Dr. Hovorka's group in Cambridge [177] and the one developed by Dr. Kovatchev and Dr. Cobelli groups in Virgina and Padova, respectively [88]. This last simulator has been accepted by the Food an Drug Administration (FDA) as a 
substitute to trials with animals in the preclinical testing of closed-loop control strategies.

Here, both simulators and the models used in them are briefly explained because these are the ones that will be used through out this work.

\subsubsection{Hovorka's group simulator [71, 177]}

The simulator developed by the group of Roman Hovorka in Cambridge University is presented in [177]. It includes 18 virtual patients represented by a model of glucose regulation [71] and their specific parameter set. The intraindividual variability is represented by super-imposing sinusoidal oscillations of $5 \%$ amplitude and a $3 \mathrm{~h}$ period on nominal values of selected model parameters. Each of those parameters had a different phase generated randomly from a uniform distribution $\mathrm{U}[0,3 h]$ [177]. The simulator also includes two types of error models for the glucose measurement and another one for characterizing insulin delivery error.

The model implemented in Hovorka's simulator has been used both for simulation and control purposes, in many different scenarios, from critical patients [73] to overnight experiments [71] with successful results. The whole model is represented in Figure 1.9 and it consists of:

\section{Insulin absorption model}

The model of subcutaneous insulin absorption is represented by three compartments, an accessible $\left(S_{1}\right)$ and a non-accessible $\left(S_{2}\right)$ subcutaneous compartment and a plasma compartment $(I)$. All of them have the same transfer rate. The model equations are:

$$
\begin{aligned}
& \dot{S}_{1}(t)=u(t)-\frac{S_{1}(t)}{t_{\max , I}} \\
& \dot{S}_{2}(t)=\frac{S_{1}(t)}{t_{\max , I}}-\frac{S_{2}(t)}{t_{\max , I}} \\
& \dot{I}(t)=\frac{S_{2}(t)}{t_{\max , I} V_{I}}-k_{e} I(t)
\end{aligned}
$$


where $t_{\max , I}$ represents the time to maximum bolus absorption, $V_{I}$ is the volume of distribution of rapid-acting insulin and $k_{e}$ represents the elimination rate from plasma.

\section{Glucose absorption model}

Hovorka gastrointestinal model is very simple. It consists of two identical compartments $\left(G_{1}\right.$ and $\left.G_{2}\right)$ with the same transfer rate:

$$
\begin{aligned}
\dot{G}_{1}(t) & =-\frac{G_{1}(t)}{t_{\max , G}}+\text { Bio } \cdot D(t) \\
\dot{G}_{2}(t) & =\frac{G_{1}(t)}{t_{\max , G}}-\frac{G_{2}(t)}{t_{\max , G}} \\
U_{G}(t) & =\frac{G_{2}}{t_{\max , G}}
\end{aligned}
$$

The model includes two parameters: the carbohydrate bioavailability (Bio) and the time it takes to reach peak appearance of glucose from the gut $\left(t_{\max , G}\right)$. In [177], the model is slightly modified considering that the glucose flux from the gut is a saturable process. In this way, $t_{\max , G}$ is defined as follows:

$$
t_{\text {max }, G}=\left\{\begin{array}{cc}
t_{\text {max }, G_{-} c e i l} & \text { if } U_{G}>U_{G_{-}} \text {ceil } \\
t_{\max , G} & \text { otherwise }
\end{array}\right.
$$

where $t_{m a x, G_{-} c e i l}=\frac{G_{2}}{U_{G_{-}} c e i l}$ and $U_{G_{-}}$ceil is the maximum glucose flux from the gut.

\section{Glucorregulatory model}

The main characteristic of Hovorka's glucoregulatory model is that it considers three remote effects of insulin on glucose kinetics: the effect on glucose transport/distribution, glucose disposal and endogenous glucose production $\left(x_{1}, x_{2}\right.$ and $\left.x_{3}\right)$. The glucose kinetics is represented by a twocompartment sub-model.

The equations that represent effects of insulin on glucose kinetics are: 

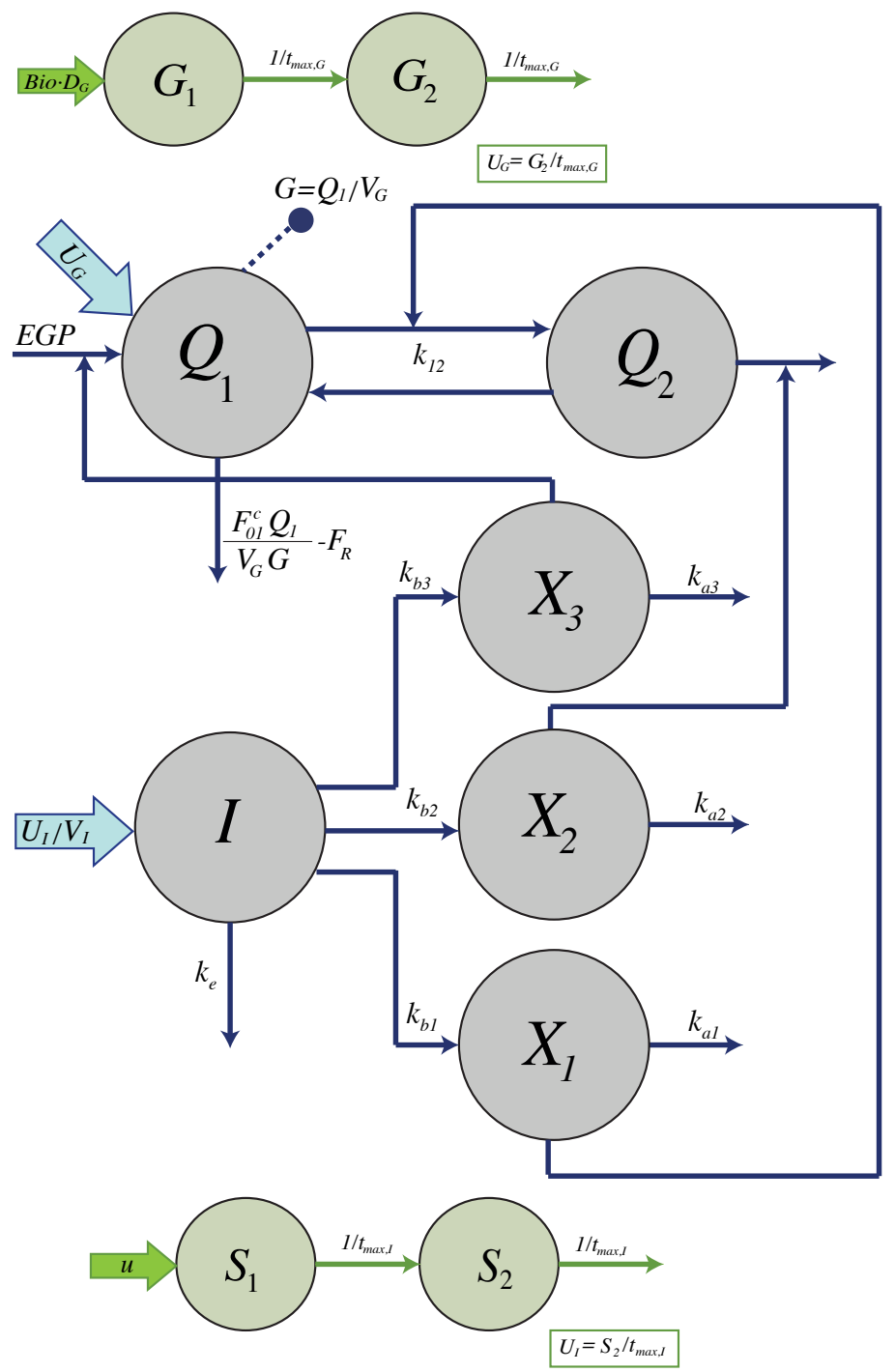

Figure 1.9: Hovorka's model implemented in the simulator. 


$$
\begin{aligned}
& \dot{x}_{1}(t)=-k_{a 1} x_{1}(t)+S_{I T} k_{b 1} I(t) \\
& \dot{x}_{2}(t)=-k_{a 2} x_{2}(t)+S_{I D} k_{b 2} I(t) \\
& \dot{x}_{3}(t)=-k_{a 3} x_{3}(t)+S_{I E} k_{b 3} I(t)
\end{aligned}
$$

$S_{I T}=k_{a 1} / k_{b 1}, S_{I D}=k_{a 2} / k_{b 2}$ and $S_{I E}=k_{a 3} / k_{b 3}$ are insulin sensitivities for transport, distribution, and endogenous glucose production, with $k_{a i}$ and $k_{b i}(i=1, . ., 3)$ representing deactivation and activation rate constants respectively.

The glucose kinetic equations are:

$$
\begin{aligned}
& \dot{Q}_{1}(t)=\left[\frac{F_{01}^{c}}{V_{G} G(t)}+x_{1}(t)\right] Q_{1}(t)+k_{12} Q_{2}(t)-F_{R}+E G P+U_{G}(t) \\
& \dot{Q}_{2}(t)=x_{1}(t) Q_{1}(t)-\left[k_{12}+x_{2}(t)\right] Q_{2}(t) \\
& y(t)=G(t)=Q_{1}(t) / V_{G}
\end{aligned}
$$

$k_{12}$ represents the transfer-rate constant from the non-accessible $\left(Q_{2}\right)$ to the accessible $\left(Q_{1}\right)$ compartment and $V_{G}$ represents the glucose distribution volume in the accessible compartment.

The endogenous glucose production is defined as:

$$
E G P=\left\{\begin{array}{c}
E G P_{0}\left[1+x_{3}(t)\right] \text { if } E G P \geq 0 \\
0 \text { otherwise }
\end{array}\right.
$$

The insulin-independent glucose utilization is assumed to be a saturable process and is represented by a Michaelis-Menten relationship:

$$
F_{01}^{c}=\frac{F_{01}^{s} G}{(G+1.0)}, \text { where } F_{01}^{s}=\frac{F_{01}}{0.85}
$$

$F_{R}$ is the renal glucose clearance above the glucose threshold of $R_{-} t h r$, and it is defined as: 


$$
F_{R}=\left\{\begin{array}{c}
R_{-} c l\left(G-R_{-} t h r\right) V_{G} \quad \text { if } G \geq R_{-} t h r \\
0 \text { otherwise }
\end{array}\right.
$$

where $R \_c l$ is the renal clearance.

\subsubsection{UVa Simulator [88, 100, 38, 37]}

The simulator developed by University de Virginia and University of Padova, was accepted by the FDA in 2008 as a substitute to animal trials in the preclinical testing control strategies. It includes a cohort of 300 virtual patients (100 adults, 100 adolescents and 100 children) based in real individual data, an in silico sensor that simulates the typical errors of continuous glucose monitoring, and a CSII pump model (see Section 2.1 and Section 2.2.1). Recently, new features have been included in the simulator mainly to improve the description of glucose kinetics in hypoglycemia [101] and to include the effect of insulin sensitivity intra-individual variability [166]. The educational version of the simulator has some limitations and only includes 30 virtual patients (10 adults, 10 adolescents and 10 children).

The insulin, gastrointestinal and glucorregulatory models used in the first version of the UVa simulator used in this work are now described:

\section{Insulin absorption model}

The insulin model proposed by Cobelli's group has two compartments for the interstitial space, and it considers that the elimination of insulin takes place entirely after the absorption into plasma. The structure of the model and the equations that govern it are shown in Figure 1.10 and equations (1.9) respectively. Note that the elimination of insulin takes places both by degradation in the plasma compartment and in the liver, which is considered as another compartment ( $I_{p}$ and $I_{l}$ respectively). 


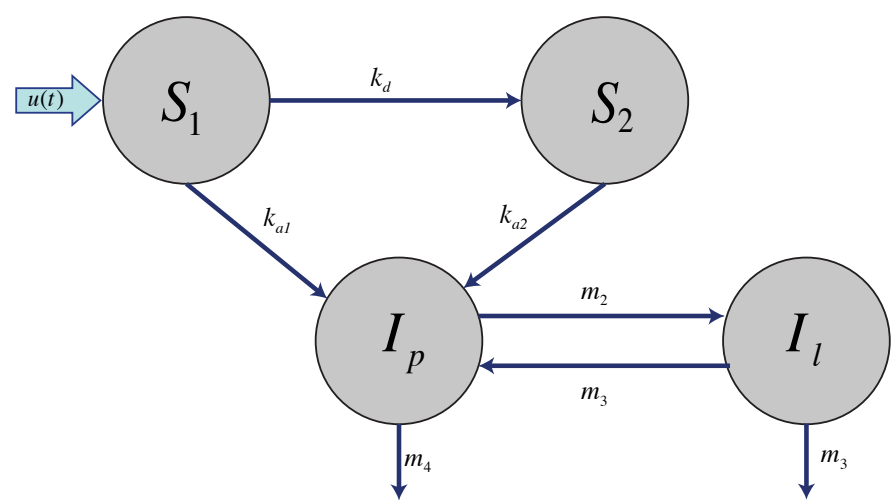

Figure 1.10: Cobelli's insulin model.

$$
\begin{aligned}
& \dot{S}_{1}(t)=-\left(k_{a 1}+k_{d}\right) S_{1}(t)+u(t) \\
& \dot{S}_{2}(t)=k_{d} S_{1}(t)-k_{a 2} S_{2}(t) \\
& \dot{I}_{l}(t)=-\left(m_{1}+m_{3}\right) I_{l}(t)+m_{2} I_{p}(t) \\
& \dot{I}_{p}(t)=-\left(m_{2}+m_{4}\right) I_{p}(t)+m_{1} I_{l}(t)+k_{a 1} S_{1}(t)+k_{a 2} S_{2}(t)
\end{aligned}
$$

\section{Glucose absorption model}

The glucose absorption model, developed by Chiara Dalla Man follows the structure shown in Figure 1.11. This model considers a two-compartment model for digestion $\left(q_{s t o 1}\right.$ and $q_{s t o 2}$ representing the amount of glucose in the stomach in solid and liquid phase respectively) and a simple singlecompartmental model for the absorption in the gut $\left(q_{g u t}\right)$.

The compartmental model equations are:

$$
\begin{aligned}
& \dot{q}_{s t o 1}(t)=-K_{21} q_{s t o 1}(t)+D \delta(t) \\
& \dot{q}_{s t o 2}(t)=-K_{\text {empt }}\left(q_{s t o}\right) q_{s t o 2}(t)+K_{21} q_{s t o 1}(t) \\
& \dot{q}_{g u t}(t)=-K_{a b s} q_{g u t}(t)+K_{\text {empt }}\left(q_{\text {sto }}\right) q_{\text {sto } 2}(t) \\
& G_{\text {ex }}(t)=f K_{\text {abs }} G_{\text {gut }}(t)
\end{aligned}
$$




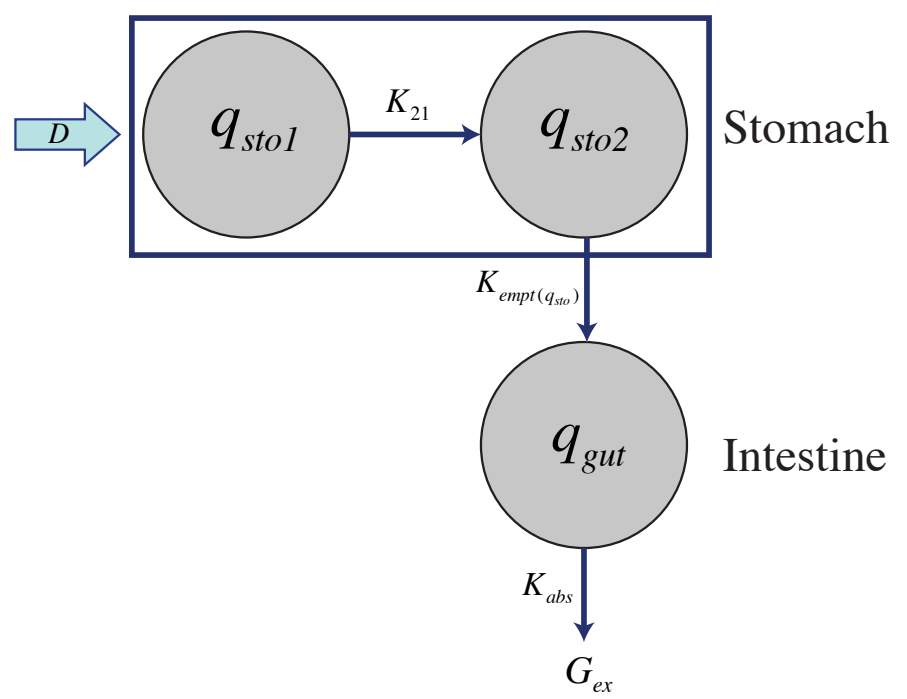

Figure 1.11: Dalla Man's gastrointestinal model.

where $\delta(t)$ is the Dirac delta, simulating an impulse input to the model. The rest of parameters are flux constants, for the transfer of glucose through the system, except for the $K_{\text {empt }}$ parameter, which is time-varying depending of the amount of glucose in the stomach $\left(q_{s t o}=q_{s t o 1}+q_{s t o 2}\right.$ and defines the form of the gastric emptying. The equations describing the transfer rate defining the flow of glucose from the stomach to the intestine are:

$$
\begin{aligned}
& K_{\text {empt }}\left(q_{s t o}\right)=K_{\text {min }}+\frac{K_{\max }-K_{\min }}{2} \cdot\left\{\tanh \left[\alpha\left(q_{s t o}-b \cdot D\right)\right]-\right. \\
& \left.-\tanh \left[\beta\left(q_{s t o}-c \cdot D\right)\right]+2\right\} \\
& q_{s t o}(t)=q_{s t o 1}(t)+q_{s t o 2}(t) \\
& \alpha=\frac{5}{2 D(1-b)} ; \beta=\frac{5}{2 D c}
\end{aligned}
$$

\section{Glucoregulatory model}

The core structure of the Cobelli glucorregulatory model is pretty simple, as shown in Figure 1.12.

The model equations are: 


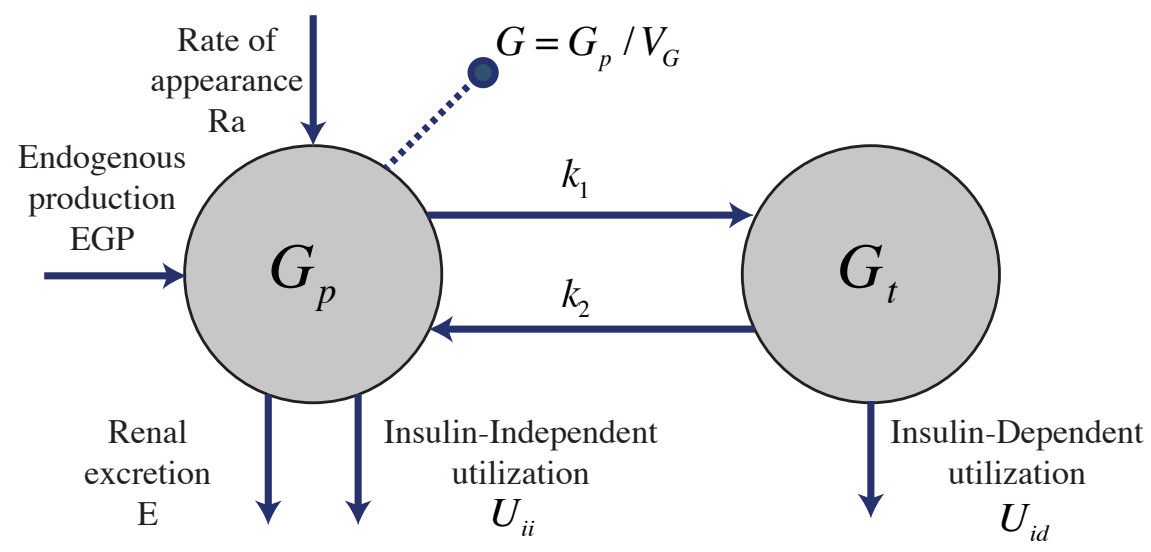

Figure 1.12: Cobelli's glucorregulatory model.

$$
\begin{aligned}
& \dot{G}_{p}(t)=E G P(t)+R a(t)-U_{i i}(t)-E(t)-k_{1} G_{p}(t)+k_{2} G_{t}(t) \\
& \dot{G}_{t}(t)=-U_{i d}(t)+k_{1} G_{p}(t)-k_{2} G_{t}(t) \\
& G(t)=G_{p}(t) / V_{G}
\end{aligned}
$$

where,

- Ra is the exogenous flux of glucose coming from the gut.

- $U_{i i}$ is the utilization of glucose that is non dependent on insulin. It is usually considered constant and equal to $F_{c n s}$.

- $U_{i d}$ is the utilization that depends on the insulin concentration, and it follows the following set of equations:

$$
\begin{aligned}
\dot{X}(t) & =-p_{2 U} X(t)+p_{2 U}\left[I(t)-I_{b}\right] \\
V_{m}(t) & =V_{m 0}+V_{m x} X(t) \\
U_{i d}(t) & =\frac{V m(t) G t(t)}{K m+G_{t}(t)}
\end{aligned}
$$


where $X(t)$ is the remote insulin, $I(t)$ is the plasma insulin, $I_{b}$ is the basal insulin and $V m(t)$ is the transfer rate for the Michaelis-Menten reaction in equation (1.13).

- $E(t)$ represents the renal excretion, which occurs if plasma glucose exceeds a certain threshold (see Section 2.1). It is modeled as follows:

$$
E(t)=\left\{\begin{array}{lc}
k_{e 1}\left[G_{p}(t)-k_{e 2}\right] & \text { if } G_{p}(t)>k_{e 2} \\
0 & \text { otherwise }
\end{array}\right.
$$

where $k_{e} 1$ is the glomerular filtration rate and $k_{e 2}$ is the renal threshold of glucose.

- $E G P(t)$ is the Endogenous Glucose Production, and it depends on a delayed insulin signal as follows:

$$
\begin{aligned}
& \dot{I}_{1}(t)=-k_{i}\left[I_{1}(t)-I(t)\right] \\
& \dot{I}_{d}(t)=-k_{i}\left[I_{d}(t)-I_{1}(t)\right] \\
& E G P(t)=\max \left\{0, k_{p 1}-k_{p 2} G_{p}(t)-k_{p 3} I_{d}(t)\right\}
\end{aligned}
$$

where $I(t)$ is the insulin concentration in plasma.

\subsection{Conclusions}

In this chapter, a quick review of the metabolism of glucose which is altered in DM has been provided together with the main characteristics of the disease. Moreover, the implications and the complications of the disease have been explained. In the same way, the main simulators that emulate the glucorregulatory system of DM1 patients were reviewed. In particular, details of the simulators developed by Dr. Hovorka's group and Dr. Kovatchev and 
Dr. Cobelli groups have been given. Those will be the base of the simulating work of this thesis.

Next chapter deals with the different strategies currently being used or tested in clinical practice in order to achieve the objective of normoglycemia. Several studies like the Diabetes Care and Complications Trial (DCCT) [61, the United Kingdom Prospective Diabetes (UKPDS) [164 and more recently, the Epidemiology of Diabetes Interventions and Complications(EDIC) [109] have demonstrated that the achievement of a good metabolic control prevents or postpones those chronic complications both in DM1 (DCCT and EDIC) and DM2 (UKPDS). For this reason, normoglycemia (blood glucose concentration within a normal range) has been established as the control objective for patients with DM, except if some contraindication exists. This is accomplished, in DM1, through the use of exogenous insulin, promoting the transport and storage of blood glucose into the cell, and thus, decreasing blood glucose concentration. 



\section{Glucose control in type 1 diabetes}

The therapeutical goals for DM are to eliminate symptoms related to hyperglycemia, reduce or eliminate the long-term complications and allow the patient to achieve as normal a lifestyle as possible. In order to achieve these objectives, each patient will need a specific treatment adapted to his characteristics (age, weight, insulin sensitivity... ). In any case, the basic pillars of the treatment are diet, exercise and, in case of DM1, an insulin therapy [14. Much effort is being put in developing new and improved technologies for insulin administration and ways to help patients to gain quality of life and to obtain a proper metabolic control.

In order to determine the proper therapy for each patient so as to keep his blood glucose levels within a established range, it is important to have short and long-term indicators that give idea of how well the patient is controlled. In Section 2.1 the main available devices and techniques to evaluate the metabolic control of the diabetic patients are summarized. Section 2.2 deals with the different kinds of insulin and the different administration possibilities that can be found in the market. A review of different open-loop insulin therapies with especial attention to intensive insulin therapies both with MDI and with CSII is provided in Section 2.3 whereas Section 2.4 deals with the different closedloop strategies that are currently being tested, remarking the challenges that arise. 


\subsection{Metabolic control monitoring}

A diabetic patient is controlled when his metabolism is not much different than a non-diabetic's metabolism. In order to achieve a good metabolic control, it is necessary to use measures to quantify the goodness of a therapy and to make adjustments in diet, exercise, and medications. In that way, monitoring of metabolic status is considered a cornerstone of diabetes care. Different metabolites are altered by diabetes but, currently, patient monitoring of capillary blood glucose is the recommended method of day-to-day testing. In this way, good metabolic control is related to the maintenance of blood glucose levels close to normality throughout the day. Besides blood glucose levels, other indices of insulin deficiency are blood and urine ketones. Moreover, measures of long-term control over the preceding weeks (glycated hemoglobin and fructosamine) are also commonly used [179, 63, 59].

\section{Capillary blood glucose}

Measuring capillary blood glucose is essential in insulin therapies. It is done with a drop of blood taken from the finger tip using a device called glucometer. The main advantage of this method is that it allows self-control since the measurements can be easily made at home. Usually, in a controlled diabetes, patients perform around four measurements in a day (one before each meal and one before going to bed) although this number can increase in some cases. Depending on the glucose measurement, the patients must apply a suitable compensatory action, that is, choose the amount of insulin to counteract the meal to be eaten and take additional compensatory meals if the glucose level is too low. This decision is based on the empirical knowledge of both the patients and the physicians.

As the major reason for poor patient compliance and for low frequency of testing is the discomfort of testing the fingers, new glucometer devices have been developed to operate at a smaller blood volume and to allow the testing at alternative body sites.

\section{Continuous glucose monitoring systems (CGMS)}


Recently, engineering and scientific advances have allowed the development of CGMS. These devices measure the glucose levels in interstitial fluid usually producing a glucose reading every 5 minutes. Data from CGMS can be presented on-line or stored for being downloaded in a computer to be analyzed. CGMS use the glucose-oxidase enzyme-based technology which gives a measurement of interstitial glucose expressed in terms of intensity of the current generated by the enzymatic reaction. They have to be calibrated using capillary blood glucose.

The most important usefulness of CGMS is that it allows to study glycemic variations within several days in order to detect hypoglycemias (specially during nights), hyperglycemia peaks, and to adjust the insulin treatment according to that. Moreover the future development of the already mentioned artificial pancreas requires accurate and frequent blood glucose measurements. However, currently, none of the existing devices have analytical accuracy comparable to that of glucometers. The potential capability of CGMS to improve glycemic control in DM1 [160] has fostered the research in CGMS to increase the accuracy of these devices, improving the technology and the calibration algorithms [11, 53, 42].

\section{Glycated hemoglobin $\left(H b A_{1 C}\right)$}

Blood glucose measurements (together with urine glucose and urine ketone testing explained later) provide useful information for day-to-day management of diabetes. However, these tests cannot provide the patient and physicians with an objective measure of glycemia over an extended period of time. Measurements of glycated proteins allow to quantify average glycemia over weeks or months.

Hemoglobin is a protein of red blood cells in charge of carrying oxygen from the respiratory organs to the rest of the body. When hemoglobin is exposed to plasma glucose, glucose molecules are attached to it forming glycated hemoglobin. Once attached, glucose molecules remain there until the red blood cell die (about 120 days). There are different types of hemoglobin and, in case of diabetes testing, $H b A_{1 C}$ is used because of the strength of the union glucose-hemoglobin. In that way, the percentage of $H b A_{1 C}$ in blood can 
be used to determine the glucose control of the patient over the last 2 or 3 months. It is recommended to combine self blood glucose measurements with regular $H b A_{1 C}$ tests. In diabetic people the ADA fixes a value of $H b A_{1 C}$ below $7 \%$ as an indicator of good metabolic control [9].

\section{Other testing methods}

Urine glucose test: The glucose urine test measures the amount of glucose in an urine sample. The presence of glucose in the urine is called glycosuria or glucosuria. It appears when blood glucose is above the renal threshold of glucose (about $180 \mathrm{mg} / \mathrm{dl}$ ). This test cannot be used by itself as a method for controlling diabetes and it has to be combined with blood glucose measurements. That is because the renal threshold is different for each person and because the test does not measure current glucose concentration but the glucose accumulated since the last urination.

Ketone bodies test: Measuring ketone bodies allows to detect a severe absence of insulin and to prevent ketoacidosis. Ketones are substances that are made when the body breaks down fat for energy because cells do not have enough glucose. This lack of glucose in the cells can be caused by an absence of glucose or by an incapability of the cells to use it. The latter is the case of diabetic people. The absence of insulin avoids the absorption of glucose by the cells and the energy needed by the organism has to be obtained from the metabolism of lipids. Ketones can be measured both in urine and in blood. The urine test is most commonly used, mainly because of its lower cost but, as it occurs with glucose tests, its accuracy is lower than blood tests.

Glycated serum protein (fructosamine) test: Glycated serum proteins are formed when serum proteins react with glucose molecules. As it happens with $H b A_{1 C}$, the concentration of glycated serum proteins reflects the average glucose level during a period of time. In the case of glycated serum protein, this period is shorter than $H b A_{1 C}$ (about 1 or 2 weeks). Because of that it is used when there has been a change in diet or treatment that requires a middleterm blood glucose measures or in special circumstances where $H b A_{1 C}$ is not suitable. 


\subsection{Insulin administration}

Patients with DM1 require exogenous insulin administration for living. Moreover, the majority of DM2 patients eventually require insulin to preserve satisfactory glucose control and an $H b A_{1 C}$ bellow $7 \%$. One international unit of insulin (1 IU) is defined as the "biological equivalent" of $34.7 \mu \mathrm{g}$ pure crystalline insulin. This corresponds to the amount required to reduce the concentration of blood glucose in a fasting rabbit to $45 \mathrm{mg} / \mathrm{dl}$ [4]. Nowadays the most common insulin concentration in the market is $\mathrm{U}-100(100 \mathrm{IU} / \mathrm{mL})$.

Until the 1980s, insulin was extracted from the pancreas of cattle and pigs. Nowadays, animal insulin is fallen in disuse and it has been substituted by synthetic human insulin, i.e., insulin with a chemical structure identical to the insulin produced by the human pancreas (Figure 2.1). The sequence of this human insulin can be modified so as to improve its pharmacokinetic characteristics (analog insulins).

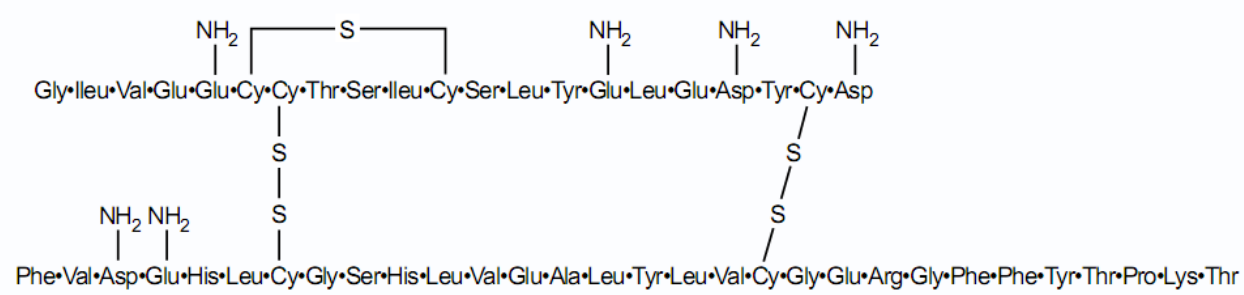

Figure 2.1: Molecular structure of human insulin.

In that way, many kinds of insulins (human and analog) can be found in the market depending on their pharmacokinetic characteristics. In general terms they can be divided into regular short-acting, intermediate-acting and longacting insulin. Additionally, mixtures of short an intermediate-acting insulin are also being use to combine the effect of both insulins. The main parameters that characterize insulin types are time of the start of the action, time of peak and duration. Figure 2.2 shows in a schematic way the pharmacokinetics of the main kinds of insulin available in the market. Remark that the effect of each kind of insulin is not always the same. The absorption can be different 
depending on the patient and even in the same patient in different moments as many factors are involved.

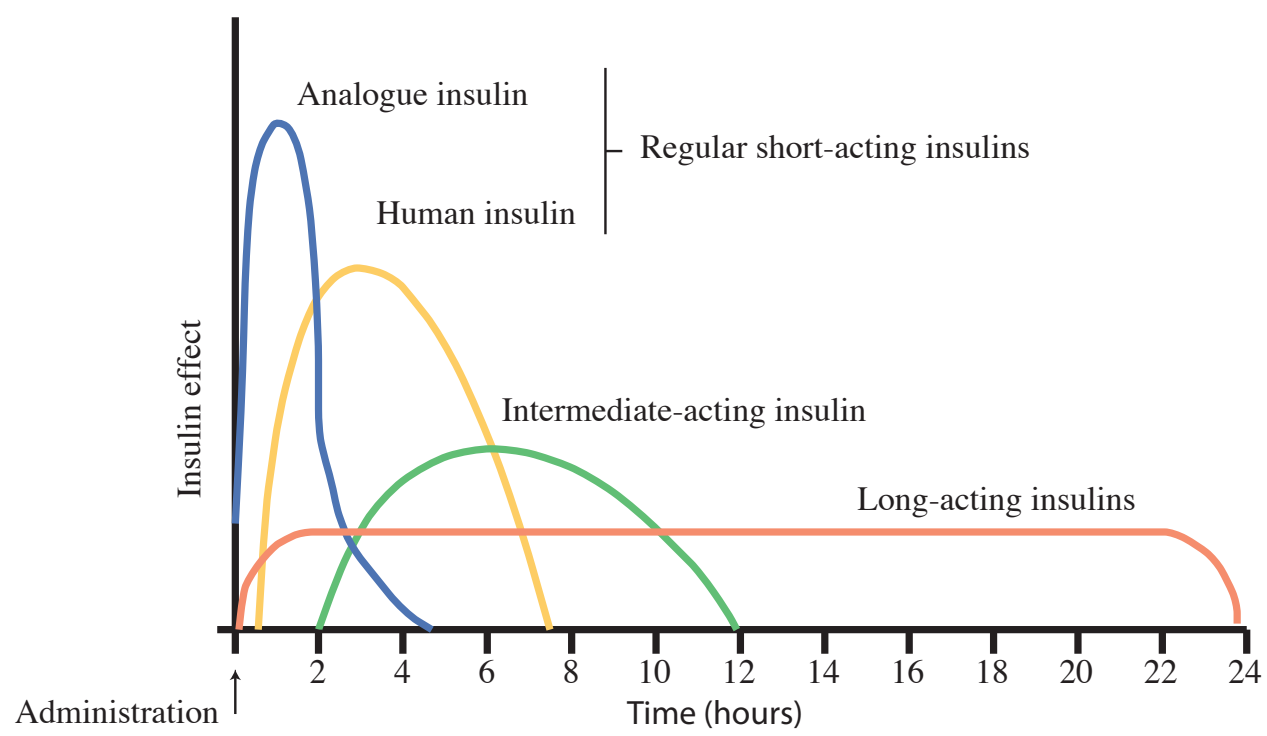

Figure 2.2: Different insulin effects. Adapted from [43].

Insulin is usually injected subcutaneously, although it can be also injected intravenously if a rapid decrease in glucose concentration is needed. Additionally, new administration methods such as inhaled insulin are being developed to avoid the annoying use of needles.

\subsubsection{Administration devices}

Traditionally, insulin has been administrated using vials and syringes but lately, they are being substituted by simpler and more convenient devices such as insulin pens. In patients for whom glucose variability remains a problem, continuous subcutaneous insulin infusion via an implanted cannula or continuous intraperitoneal insulin infusion via an implanted pump is safe and effective when used correctly, although cost can be a limitation. Less invasive devices are jet injectors and the devices for inhaled insulin [120]. A brief description of the possibilities for insulin administration is provided: 
Vials or syringes: Syringes have been used for insulin administration since 1960s and are still the main used device in many countries. They have the advantage compared with insulin pens that they allow patients to mix their own insulin formulations. However, the existence of premixed insulin analogs for use in insulin pens is fostering the replacement of vials with pens due to their highest accuracy and patient adherence.

Insulin pens: Several studies have shown that insulin pen devices have several advantages over the traditional vial and syringe methods, including improved patient satisfaction and adherence, greater ease of use, and superior dosing accuracy [119]. These devices consist of an insulin cartridge or reservoir and a syringe and are available as either reusable or disposable types. Pen devices are more accurate than syringes especially at doses below 5 IU.

Jet injectors: Jet injectors use high pressure to send a fine spray of insulin through the skin. They are specially recommended for those patients with needle-phobics.

Continuous subcutaneous insulin infusion (CSII) pumps: CSII pumps are external devices that allow the administration of insulin in a continuous way. They have an insulin deposit which is filled in the same way than a normal syringe. The pump is attached to a small tube or catheter with a needle on the end that is inserted in the skin, usually in the abdomen and that has to be changed every three days. These pumps use short-acting insulin to infuse, in a continuous way, a previously programmed basal insulin profile needed to keep blood glucose within the desirable range between meals and in the night period. Besides, the patient can indicate the extra amount of insulin needed to counteract each meal (bolus insulin). The newest models of CSII pumps have several advanced features, such as the incorporation of bolus advisors and, the possibility of implementing alarms when they are coupled to CGMSs or even an automatic pump shut-off when hypoglycemia is detected. However, these devices are not yet automatic and it is still the patient who has to determine the insulin dose for each meal [142].

The main advantage of CSII pumps is that the continuous infusion of shortacting insulin allows different profiles of basal insulin improving flexibility and 
that insulin can be dosed in smaller increments than in pens or syringes $(0.1$ $I U)$. It is specially useful in patients experiencing severe hypoglycemia or hypoglycemia unawareness. On the contrary, patients using insulin pumps are more likely to suffer diabetic ketoacidosis (see Section 1.3) due to the use of short-acting insulin that remains less time in the subcutaneous compartment. However, training of the patient and frequent self-monitoring of blood glucose reduce this risk [146].

Recently, a new type of CSII pump has been developed. The catheter that joins the insulin pump and the place of infusion is eliminated and the pump is directly connected to the skin with an adhesive patch. The main advantages of this kind of pumps are that they are small, lightweight and can be manipulated discreetly. However they still have some problem such as poor adherence or adhesive intolerance by the patient [8].

Continuous intraperitoneal insulin infusion (CPII): An alternative to CSII is CPII where insulin is delivered through an implantable pump that allows more predictable insulin profiles and improves glycemic control. Because of the high costs and the lack of experience with these devices their use is still limited to a very specific patients.

Inhaled insulin: One of the possibilities that is being explored in pharmaceutical industries is the use of less invasive insulin administration modes. In September 2006 the first inhaled insulin was put in the market by the pharmaceutical Pfizer but its selling was interrupted due to poor acceptance from patients and providers. Currently, there is one formulation of inhaled insulin in phase III clinical trials called Afrezza. With this technology insulin particles are delivered through an inhaler into the lungs and enter the bloodstream through tiny blood vessels.

Figure 2.3 shows the different external devices that are used for insulin administration. 


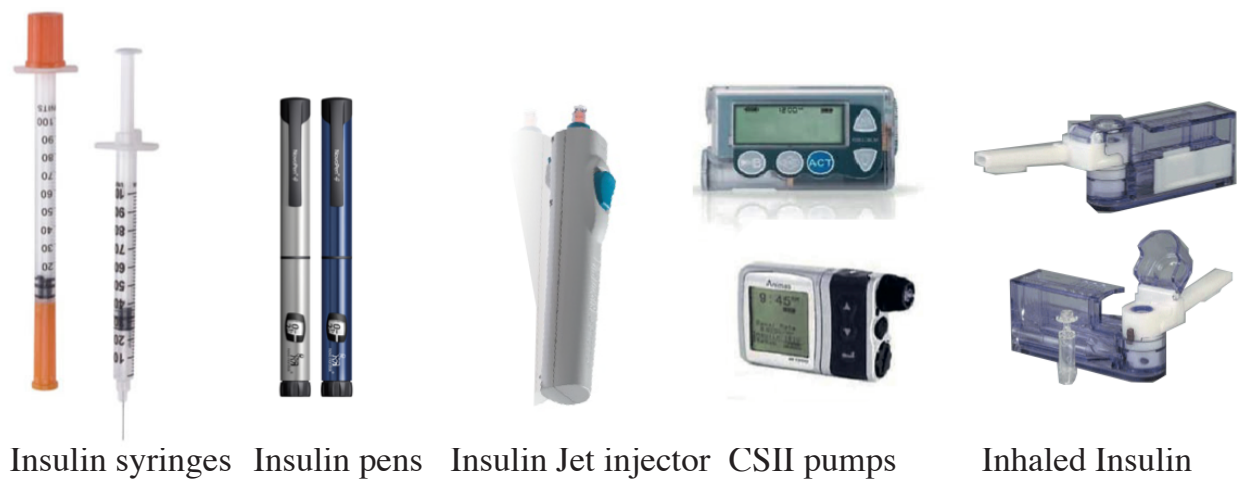

Figure 2.3: External insulin administration devices.

\subsection{Open-loop glucose control in type 1 diabetes: intensive insulin therapy}

In order to establish an insulin regimen for DM1, different factors have to be taken into account. In that way, depending on the patient's age, the ability to understand and implement a complex regimen, the presence or not of complications, the ability to recognize hypoglycemic symptoms, the lifestyle, etc,... physicians can choose either a conventional insulin therapy or an intensive insulin therapy.

The conventional insulin therapy consists of one or two injections per day of long-acting or intermediate-acting insulin. The objective of this kind of therapies is to keep the patient asymptomatic, preventing extreme decompensations. In that way, the control objective is less ambitious than with an intensive therapy allowing $H b A_{1 C}$ values between 8 and $9 \%$. This insulin regimen is relatively easy to understand and it does not require high training. Because of that, it is often recommended to very young or very old patients or patients with severe hypoglycemia problems. Conventional regimens are also frequently used in DM2, with patients that cannot be controlled using oral agents.

However, in most cases, in order to obtain good glucose control patients have to follow an intensive insulin treatment. The objective of this insulin 
regimen is to mimic a normal insulin secretion pattern. In order to achieve this objective a basal-bolus strategy is used. Basal insulin is used to keep glucose in normoglycemia during the night period and between meals whereas bolus insulin is used to counteract the different meals.

Intensive insulin therapy allows greater flexibility in meal timing and amounts but it requires greater motivations and skills from the patient. To be successful, it requires frequent blood glucose monitoring (3-5 times per day). This kind of therapy is indicated for adult patients and selected adolescents and children with DM1 and for treating gestational diabetes.

\subsubsection{Intensive insulin therapy: MDI and CSII}

The study developed by the DCCT in 1993 61 finally confirmed the superiority of intensive insulin therapy in DM1 in conjunction with frequent selfmonitoring of blood glucose in reducing the long-term diabetes complications, compared with conventional therapy. Intensive insulin therapy can be based on MDI or in CSII using insulin pumps. Moreover, it has to be adapted to the specific characteristics of each patient adjusting the insulin regimen to each one.

MDI: MDI is the most popular regimen for intensive insulin therapy. In MDI the basal insulin is provided by intermediate or long-acting insulins and short-acting insulins are used before each meal.

A common insulin regimen consists of administering two daily injections of intermediate-acting insulin mixed with short-acting insulin (or a premixed insulin) before the breakfast and the dinner (Figure 2.4 A). Usually, two-thirds of the insulin is given in the morning and the rest before the dinner. The problem of this kind of therapy is that patients have to follow a strict schedule of meals and daily activity because of its poor flexibility.

Figure 2.4 B shows a different intensive insulin therapy where short-acting insulin is injected before each meal (bolus insulin). This kind of regimens are more flexible and allow adjustments in the bolus dose depending on the glucose concentration before the meal and on the type of meal. The basal insulin can 
Morning Afternoon Evening Night

(A)

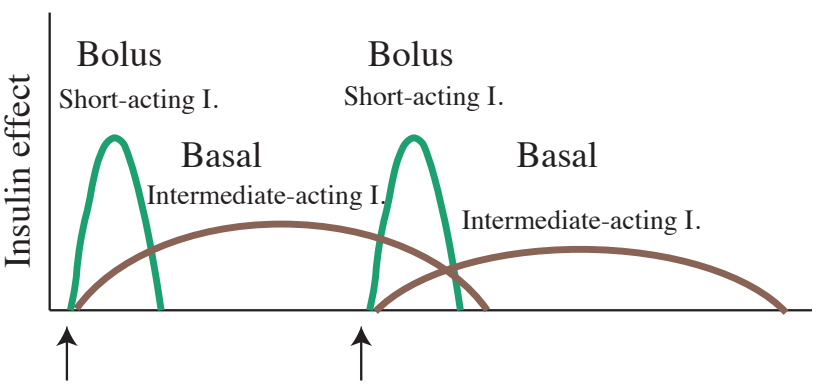

B

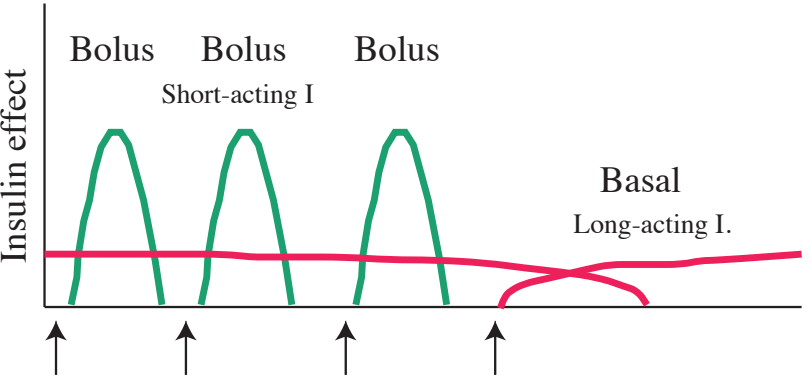

(C)

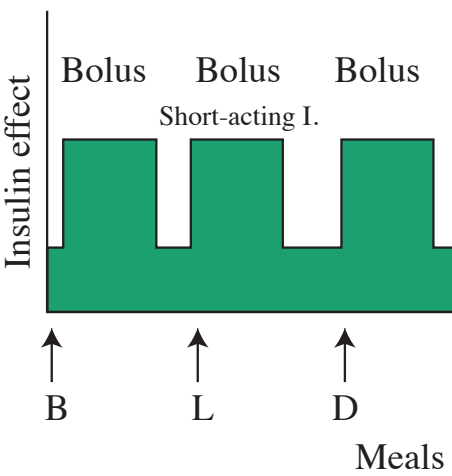

Figure 2.4: Different intensive insulin therapy regimens. Adapted from [81]. The $\mathrm{x}$-axis shows the time of the day: $\mathrm{B}$, breakfast; L, lunch and $\mathrm{D}$, dinner. Vertical arrows indicate the time of insulin injection/bolus infusion. 
be administered using one long-acting insulin injection or two intermediateacting insulin injections. In order to succeed in this kind of regimen home blood glucose monitoring is essential, specially before each meal.

CSII: Intensive therapy can also be administered via CSII, through portable electromechanical pumps (see Section 2.2.1). Insulin pumps use continuous short-acting insulin infusions to administer basal insulin. This fact allows to program different basal profiles depending on the known changes in insulin sensitivity of a patient along the day (see Figure 2.4 C ). Multiple basal infusions rates can be programed to accommodate nocturnal versus daytime basal insulin requirements, alter infusions rate during periods of exercise or select different waveforms of insulin infusion. In that way, phenomenons such as the "dawn phenomenon" "1] can be counteracted. Indications for CSII include patients unable to achieve glycemic targets with MDI and motivated patients who indicate a preference for CSII over MDI. It often requires technical support for the pump and a diabetes care team trained in pump usage.

Extensive research has been conducted over the years to evaluate the clinical effectiveness of CSII compared with MDI [19, 67, 127, 44]. In general CSII achieves slightly improved mean blood glucose concentration and $H b A_{1 C}$ values. The frequency of hypoglycemia is usually lower in CSII.

Since the first commercial insulin pumps marketed in the late 1970s, many improvements have been developed. Most modern pumps, termed "smart pumps", have tools to aid diabetic subjects in the prandial bolus decision-making process: the bolus advisors. They take into account the patient's current blood glucose level, target blood glucose level, amount of carbohydrate to be consumed, insulin-to-carbohydrate ratio (I:C) ${ }^{2}$, and an approximation of the insulin action curve to determine the amount of insulin remaining in the body from previous insulin boluses [184]. Several studies have demonstrated superior postprandial performance of automated bolus

\footnotetext{
${ }^{1}$ An early morning increase in blood sugar concentration caused by the release of counterregulatory hormones such as growth hormone, cortisol, glucagon, or epinephrine.

${ }^{2} \mathrm{~A}$ ratio that specifies the number of grams of carbohydrate covered by each unit of short-acting insulin. This ratio, determined by the physician for each patient, serves as the foundation for adjusting premeal bolus insulin doses.
} 
calculation against manual bolus with a high degree of satisfaction by the patient [83, 60, 147] reflecting better insulin dosing accuracy and ease of calculations. However, a positive impact on long-term glucose control $\left(H b A_{1 C}\right)$ has not been demonstrated. In order to be efficient, the parameters of bolus calculators have to be well tuned. Incorrect tuning of the I:C ratio, the correction factor to be applied if glucose concentration is abnormally high or low, and the duration of insulin action is a limitation for the success of CSII therapies [172, 173].

Another feature of smart insulin pumps is the ability to change the shape of the boluses to account for differences in meal absorption with respect to nutritional content and variable needs of insulin [115]. Currently available bolus shapes or waveforms are standard wave, square wave, and dual wave. With the standard wave all the bolus insulin is delivered in the briefest period, with the square wave the bolus is delivered over an extended period of time, while a dual wave bolus mode is a combination of both. It is used to deliver part of the bolus immediately and the reminder over a period of time. In Figure 2.5 a schematic representation of the different available bolus waveforms is shown. Each of these waves may provide better glucose control depending on the amount, duration, and composition of the meal. Square wave and dual wave profiles have been shown to be more effective than standard bolus in controlling postmeal glucose excursion in slow-absorption meals and meals with high fat content [84, 31, 79, 116]. In [171] and later in [20] an additional waveform called "super-bolus" which administers future basal insulin at the bolus time to compensate hyperglycemia quicker is proposed.

Moreover, as first step to automatic control, some new insulin pump devices are linked to a CGMS allowing the implementation of new features such as the automatic pump suspension when CGMS alerts to a low glucose concentration [33, 25].

However, despite the availability of these new tools, achievement of good metabolic control in terms of $H b A_{1 C}$ is still an elusive goal in more than $50 \%$ of patients with T1DM due, among other things, to clinical inertia and patient's low compliance [83, 172, 173]. In fact, optimization of postprandial control is 
still an empiric process based on the experience of both the physician and the patient.

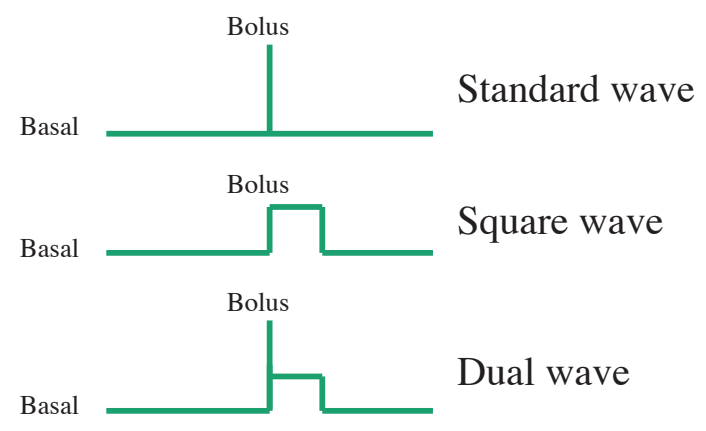

Figure 2.5: Graphical representation of different bolus waveforms implemented in insulin pumps.

\subsection{Closed-loop glucose control in type 1 diabetes: Artificial Pancreas}

In an attempt of improving quality of life of DM1 patients, and to reduce their implication in insulin dosing decisions, much effort has been put in developing systems for administering insulin automatically. Closing the loop between the glucose sensors and the insulin pumps using control algorithms (the so-called artificial pancreas) has been a dream of the scientific community for more than 40 years.

Although some research is still being done using alternative delivery routes [134, most of the closed-loop strategies currently being tested use the subcutaneous route both for the glucose sensor and the insulin administration. However, the first approaches of the artificial pancreas measured glucose and delivered insulin and glucose intravenously. The first closed-loop insulin pump device was designed in 1964 by Kadish [80]. The control algorithm was an "on-off system" that administered insulin if the blood glucose was above 150 $\mathrm{mg} / \mathrm{dl}$, and glucose if blood glucose was below $50 \mathrm{mg} / \mathrm{dl}$. Despite its huge 
size (see Figure 2.6) which made the system unfeasible for daily use, and its simple control algorithm this first approximation fostered the development of improved systems.

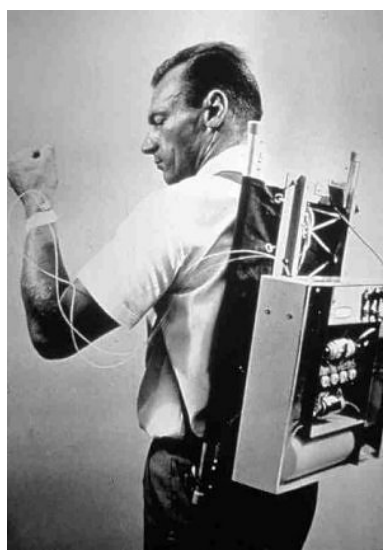

Figure 2.6: First insulin pump developed by Kadish in 1964.

During the 1970s, several research groups presented closed-loop control results, standing out the works of Albisser et al. [5] in Toronto and Pfeiffer et al. in Ulm [123] whose results led to the first commercial closed-loop device called Biostator [35] put into production in 1977 (currently relegated to hospital research); and the work of Shichiri et al [148] that was implemented in the Nikkiso STG-22 device [182] (an evolution of this device is being used in Japan in clinical practice [163]). Both devices used proportional-derivative (PD) controllers.

Pickup [126] and Tamborlane [155] first demonstrated the feasibility of the subcutaneous route for insulin delivery but, the improvements in CSII technology in the last two decades and the introduction of the first commercial minimally invasive subcutaneous glucose sensing in 1999 [105] have accelerated the research in a wearable subcutaneous-subcutaneous artificial pancreas. Despite its inherent challenges, subcutaneous route has been elected in most of the recent closed-loop systems because of its management and safety at the expense of more invasive routes such as intravenous or intraperitoneal.

Figure 2.7 shows and schematic representation of a glucose closed-loop 
system using subcutaneous route. In these systems, the measured output is the subcutaneous glucose provided by the CGMS which is an estimation of plasma glucose. The control variable is insulin administered via CSII pumps, and the main disturbances to be counteracted are meals and exercise.

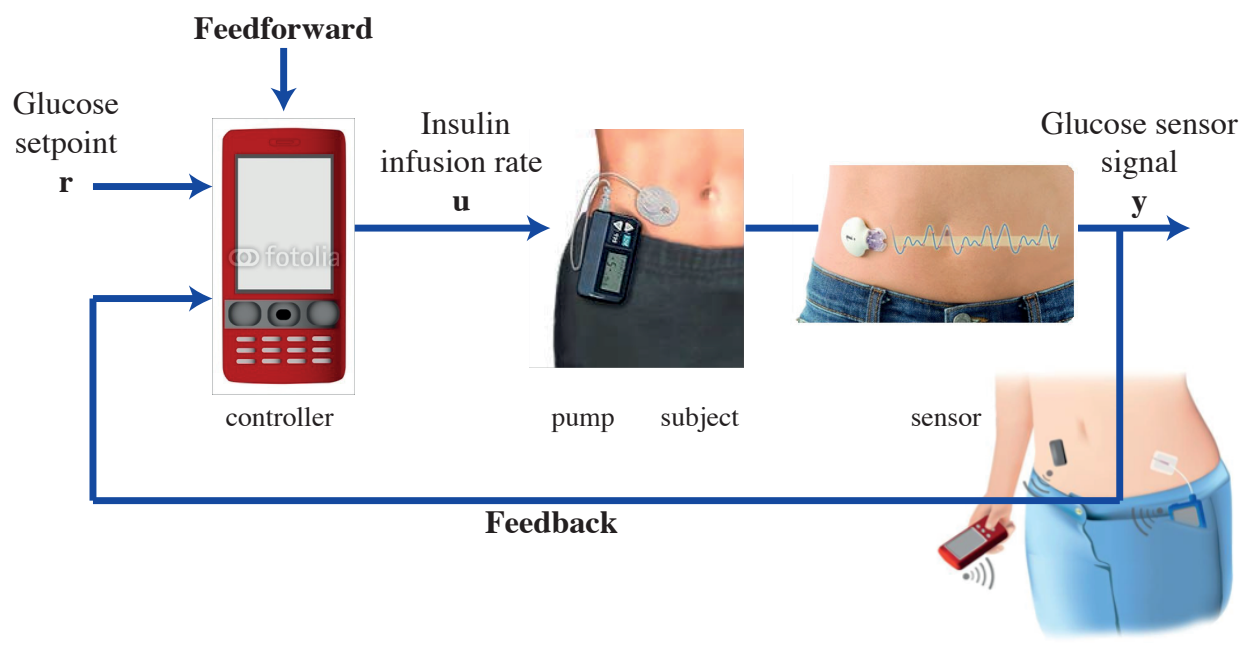

Figure 2.7: Schematic representation of a glucose closed-loop system using the subcutaneous route. Adapted from [70] and [15].

A review of the main challenges that have to be overcome in the development of an artificial pancreas using the subcutaneous route is provided in next section.

\subsubsection{Main challenges}

As it has been already mentioned, depending on the implementation devices used, different routes can be found for closing the loop (see Table 2.1). Due to its minimally invasive nature, the most commonly used route currently is subcutaneous both for sensing and delivery. This route, however, adds additional problems to the already complicated task of developing an automatic blood glucose controller, mainly because the delays in insulin action. In Figure 2.8 , the delays associated with each closed-loop route are showed.

The main challenges for the development of the artificial pancreas are: 


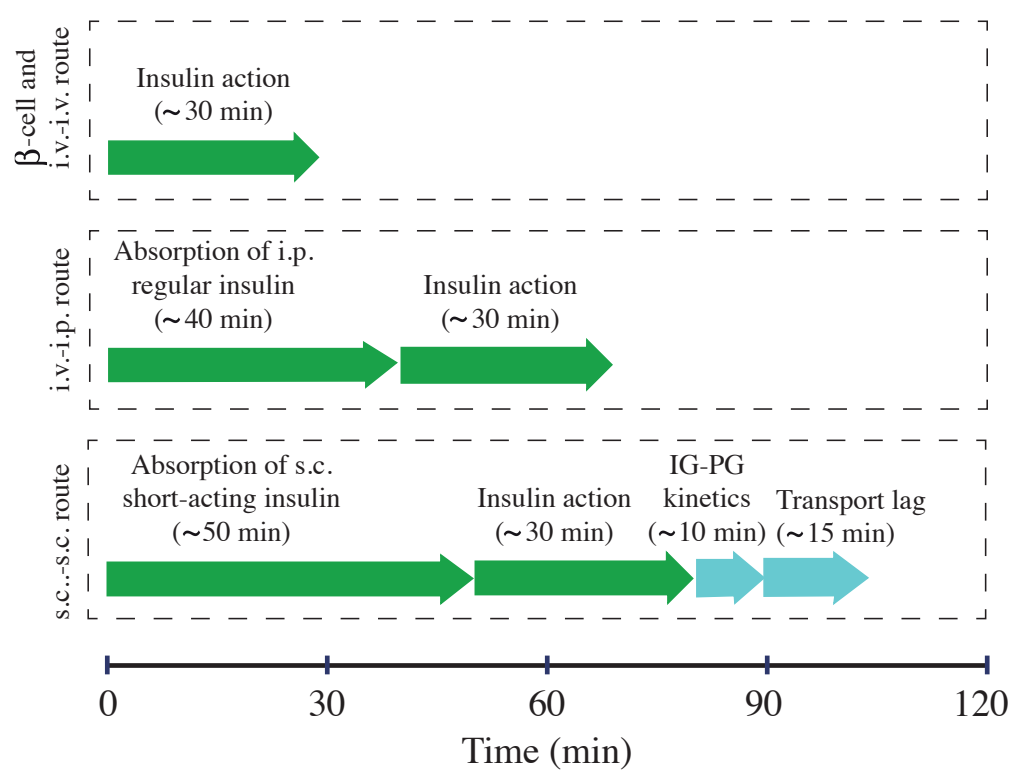

Figure 2.8: Delays in insulin action depending on the route used. Adapted from [68]. Last transport lag is only present in microdialisys or microperfusion-based systems. 
Table 2.1: Artificial Pancreas routes. Adapted from 22.

\begin{tabular}{|l|l|l|}
\hline $\begin{array}{l}\text { Glucose } \\
\text { measurement }\end{array}$ & Glucose Infusion & Characteristics \\
\hline i.v & i.v & $\begin{array}{l}\text { The absence of delays allows for rapid changes if needed. } \\
\text { But, it is only viable in intensive care units. }\end{array}$ \\
\hline i.v & i.p. & $\begin{array}{l}\text { Insulin action closer to physiology than s.c infusion. The } \\
\text { cost of the infusion devices together with the necessity of } \\
\text { implantation represent the main drawbacks. }\end{array}$ \\
\hline s.c. & i.p. & $\begin{array}{l}\text { Glucose monitor minimally invasive and implantable pump. } \\
\text { Same drawbacks than the i.v.-i.p. route plus additional ones } \\
\text { derived of the sensor errors. }\end{array}$ \\
\hline s.c & s.c. & $\begin{array}{l}\text { Glucose monitor minimally invasive and external pump. } \\
\text { Easy to manipulate. The non physiologic infusion of insulin } \\
\text { adds delays in its action. }\end{array}$ \\
\hline
\end{tabular}

i.v., intravenous, i.p., intraperitoneal, s.c., subcutaneous

\section{Insulin delivery}

The principal problem of subcutaneous insulin delivery is the presence of delays, that, in spite of using short-acting insulins, can be higher than $80 \mathrm{~min}$ (50 min for insulin absorption and $30 \mathrm{~min}$ for insulin action) [68]. Moreover, there can be significant variability in the pharmacodynamic action of insulin from one patient to another [47] and even in the same patient along the day. These facts difficult the reduction of glucose peaks after a meal and, moreover, the delayed insulin action when postpandrial glucose is already decreasing, may result in later hypoglycemias.

An additional problem of CSII pumps is the presence of mechanical failures such as occlusions, inflammation of the zone of infusion or insulin leakage back to the skin surface.

\section{Perturbations}

The main perturbation in a closed-loop glucose system is meals. They are large and uncertain perturbations difficult to counteract due, among other things, to their faster response in glucose concentration as compared to insulin delivery. Although clinical studies have demonstrated better performance in feed-forward control strategies ("meal announcement") [176], the amount of carbohydrates in a meal is no always easy to calculate. In addition, it must be taken into account that the effect of a meal in the blood glucose 
depends on many factors, not only on the ingestion time, and the grams of carbohydrates. Factors such as the nutritional composition of the meal, the way it has been cooked and the effect of previous meals make the meal a no completely measurable perturbation.

\section{Variability}

Another important issue that must be considered when thinking in glucose control is variability. This variability is not only referred to inter-patient variability (among patients), but also to changes in the insulin sensitivity in the same patient along a day. Insulin sensitivity is known to have circadian variations of different intensity depending on the patient [168]. Other sources of intra-patient variability are stress, exercise, infections...

\section{Glucose measurement}

One of the biggest challenges for the artificial pancreas is the lack of enough accuracy of current CGMS with Mean Absolute Relative Difference (MARD ${ }^{3}$ ) close to $15 \%$ [92]. CGMS failures are related to the complex interaction between the sensor and the subject. Motion of the patient, pressure on the sensor site, and foreign body response to the sensor, among others things, influence the output of the CGMS. Typical sensor failures are signal loss and sensor drifts. As CGMS give measurements of glucose concentration in the interstitial fluid, they have to be calibrated using capillary blood glucose measurements. Calibration algorithms currently used in commercial CGMS are based in linear regression methods and present limitations [141]. Moderns calibration algorithms are being suggested in literature [12, 94, 53, but are not still validated in clinical context. The fact of measuring in interstitial fluid adds also delays that are considered in Figure 2.8.

Because of all the difficulties that have to be overcome, achieving a proper closed-loop glucose controller is not an easy task. Partial solutions helping to improve control and patients' quality of life mean a big step in the final development of an artificial pancreas. In next section, an overview of the

\footnotetext{
${ }^{3}$ The result of a mathematical calculation that measures the average disparity between the sensor and the reference measurement.
} 
current state of automatic blood glucose control algorithms is provided, paying especial attention to those strategies with more clinical evidence.

\subsubsection{Artificial pancreas nowadays}

In the last decade, many control algorithms have been proposed and tested in simulations (in silico) such as $H_{\infty}$ [117, 143, 130], sliding mode control with glucose prediction after meals [54, neural networks and fuzzy logic [154, 28], adaptive control structures [76] and algorithms inspired in the molecular biology of beta cells [113 but those with the best clinical evidence of efficacy are proportional-integral-derivative (PID) [153, 103] controllers and model predictive control (MPC) [71].

PID algorithm, adapted from control systems in industrial sector, tries to emulate the behavior of $\beta$ cells [152 calculating insulin delivery based on three terms. The proportional $(\mathrm{P})$ term is directly related to the the glucose error (current glucose concentration minus glucose target). The integral (I) component adjusts insulin delivery according to the area under the curve between measured and target glucose (accumulated error). The derivative (D) term is based on the rate of change of blood glucose over time (error trend).

The typical equation of a PID glucose controller is:

$$
u_{c}(t)=k_{P} e(t)+k_{D} \dot{G}+k_{I} \int_{0}^{t} e(t) \mathrm{d} t
$$

where $e(t)=G(t)-G_{r}(t)$ with $G_{r f}(t)$ the glucose reference and $k_{P}, k_{D}$ and $k_{I}$ the constants of the proportional, derivative, and integral parts of the PID respectively. Figure 2.9 is an schematic representation of the effect of each PID part in response to a hyperglycemic clamn 4 .

The most relevant achievements using PID algorithms are from Medtronic Diabetes and its ePID insulin infusion system [153].

\footnotetext{
${ }^{4} \mathrm{~A}$ method used usually for quantifying insulin secretion and resistance that consists in maintaining a high blood sugar level by perfusion or infusion with glucose.
} 


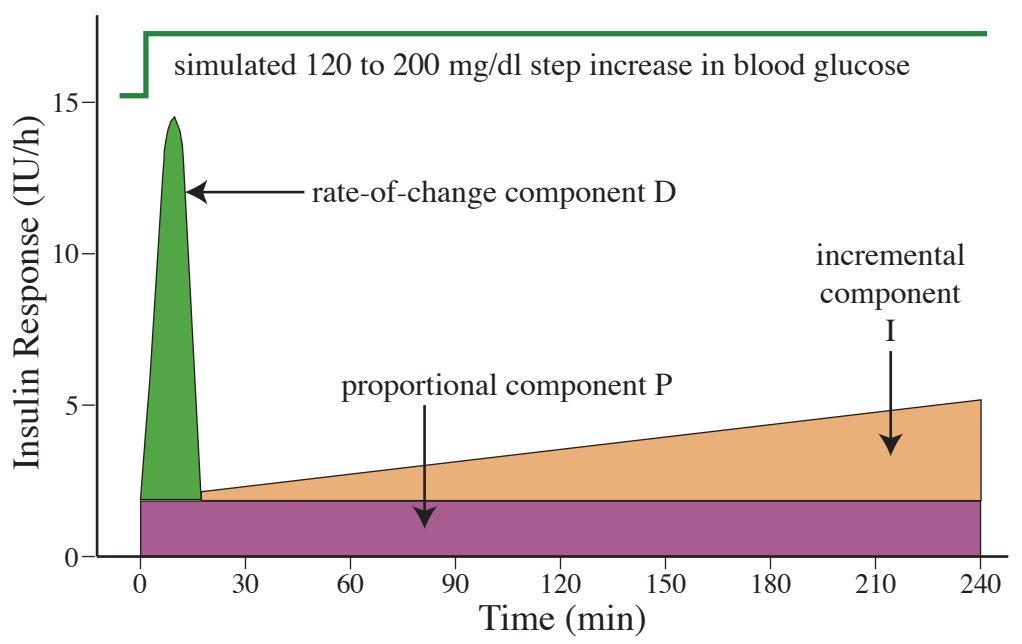

Figure 2.9: Response of each PID part to a hyperglycemic clamp. P component is proportional to the error, I component increases if the time outside the target increases and D component is active when there is an increment in blood glucose [153.

The MPC approach uses mathematical models to predict the glucose concentration response to insulin delivery. The control action is obtained by solving, at each sampling time, a finite horizon optimal control problem (see Figure 2.10 . In that way, the main ingredients of MPC are the model, the cost function and the constraints, which are accounted for during the control law design. The main potential advantage of MPC is prediction capability, but, as a drawback, this prediction and the whole performance of the controller are very dependable on the model goodness.

The main contributions of these approaches are from University of Cambridge [73, Universities of Virginia and Padova [100, and the University of California Santa Barbara in collaboration with the Sansum Diabetes Research Institute [52].

Another approach which is currently being tested in clinical trials is an algorithm that applies the principles of fuzzy logic theory to imitate the way of reasoning of diabetes caregivers [10, 110].

An ideal glucose controller would be one capable of limiting the insulin 


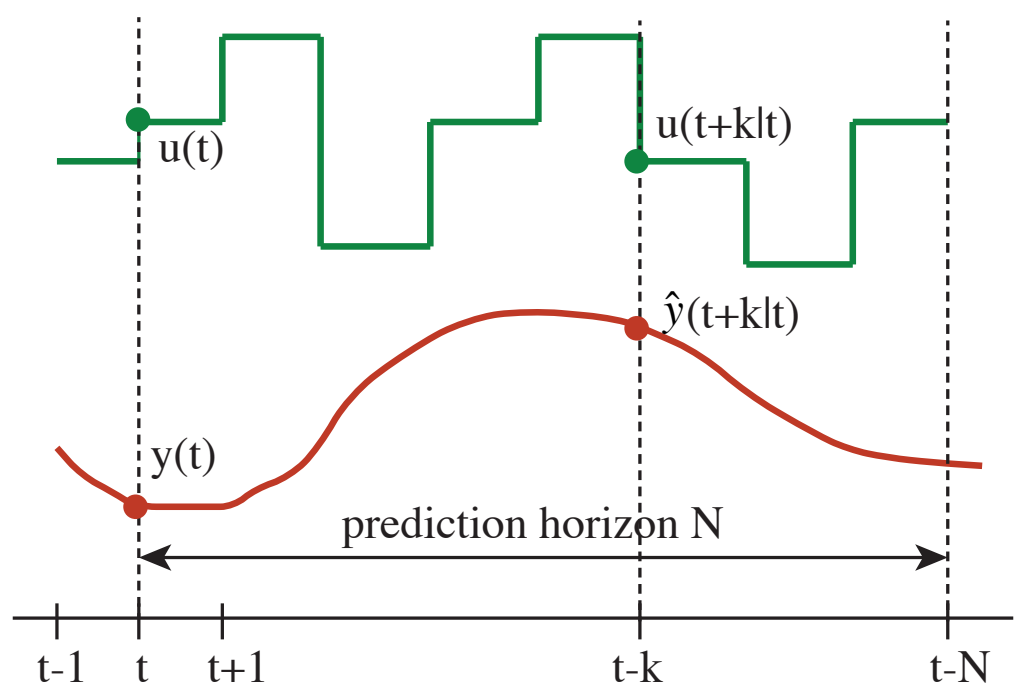

Figure 2.10: MPC way of operation [71]. Based on a model, the output trajectory $\hat{y}(t+t \mid k), k=1, \ldots, N$, is estimated for any given control sequence $u(t+k \mid t)$ over a prediction horizon $\mathrm{N}$.

infusion, minimizing the hypoglycemia events, and robust in the presence of intra-patient variability and errors in the measurements. In addition, it cannot be too complex, and needs to be adjustable according to clinical practice. However, its development is not easy at all, due to the challenges and difficulties listed in Section 2.4.1

In [90] a roadmap to a closed-loop artificial pancreas is provided. It consists of 6 stages: (i) pump shut-off to avoid hypoglycemia, (ii) a predictive hypoglycemia minimizing system, (iii) a system that controls glucose between low and high glucose limits, (iv) overnight control to a desired glucose setpoint, (v) fully closed-loop control using insulin, and (vi) fully closed-loop control using both insulin and glucagon. Apart from the first stage which has already entered in the postmarketing stage with the low glucose suspend function integrated in the Paradigm $\AA$ Veo (Medtronic) insulin pump [125], all the rest are still in research phase.

In the last years many clinical trials have been carried out mainly after 
the Juvenile Diabetes Research Foundation (JDRF) launched the Artificial Pancreas Project in 2006 [2], setting up a consortium of Universities and other research organizations to promote collaboration in diabetes research 5 . This consortium, together with DREAM [110] and Artificial Pancreas at home (AP@home) [1] consortiums, have monopolized the list of the most recent clinical trials. The approval by the Food and Drug Administration of the UVa simulator [88] (Section 1.4.2), that substitutes animals testings before human trials, has accelerated also the validation of the algorithms.

The main approaches already proposed and those that are currently being tested, both for overnight and postprandial control are explained now.

\section{Overnight control}

According to [158], most severe hypoglycemic episodes occur during sleep. The possibility of reducing these events by using a closed-loop strategy is promising due to the absence of the biggest perturbations (meals and exercise). Several studies have been developed by Cambridge University to evaluate the efficacy of an MPC-based closed-loop algorithm overnight [93] with promising results. The algorithm was evaluated in hospital in randomized crossover studies in 17 children and 24 adults. Time in target plasma glucose $(70-144$ $\mathrm{mg} / \mathrm{dl}$ ) in both young and adults was longer than using a conventional CSII therapy (from $40 \%$ to $60 \%, \mathrm{p}=0.002$ in young and $50 \%$ to $76 \%, \mathrm{p}<0.001$ in adults). Moreover, glycemic variability was also lower for closed-loop (27 versus $37.8 \mathrm{mg} / \mathrm{dl}, \mathrm{p}=0.007)$.

Another study using an MPC controller developed using UVa simulator 87] and carried out with 20 DM1 adults in Virginia, Padova (Italy) and Montpellier (France), showed a reduction in nocturnal hypoglycemia (blood glucose below $70.2 \mathrm{mg} / \mathrm{dl}$ ) from 23 to 5 episodes $(\mathrm{p}<0.01)$. Additionally, the fuzzy logic based MD-Logic Artificial Pancreas (MDLAP) [10] was also tested in a 12 DM1 patients cohort crossover trial conducted in Slovenia, Germany, and Israel [111. The percentage of time spent in normoglycemia $(63-140 \mathrm{mg} / \mathrm{dl})$ was significantly higher in the overnight closed-loop sessions (76\%) than during

\footnotetext{
${ }^{5}$ Go to http://jdrfconsortium.jaeb.org/ClinicsListing.aspx for an updated list of consortium participants.
} 
CSII therapy $(29 \%)(\mathrm{p}=0.02)$. The mean overnight glucose level was reduced by $36 \mathrm{mg} / \mathrm{dl}$ with closed-loop insulin delivery $(\mathrm{p}=0.02)$ with a significantly less glucose variability when compared with the CSII nights $(\mathrm{p}<0.001)$.

\section{Daily control and meal counteraction}

As explained in Section 2.4.1, the biggest challenge of glucose control is the counteraction of meals and the risk of late-postprandial hypoglycemia due to the delays in subcutaneous insulin absorption. First trials using fully automatic glucose controllers showed limitations in the postprandial control 153. with glucose peaks higher than desired (189, 172 and $225 \mathrm{mg} / \mathrm{dl}$ for lunch, dinner and breakfast respectively). The use of an hybrid approach with manual delivery of prandial insulin boluses has reported better results both with PID [176] and MPC [49]. However, late postprandial hypoglycemia continues being a problem even with meal announcement strategies [34, 24, 51].

In an attempt to add safety against hypoglycemia to closed-loop strategies, different approaches have been proposed. In [151 feedback of a model predicted insulin profile was added to the ePID algorithm (ePID-IFB) to try to better emulate the $\beta$-cell physiology, and improve postprandial behavior. The trial which included a fixed $2 \mathrm{IU}$ prebolus before each meal evaluated the feasibility of the approach with 8 patients receiving closed-loop insulin delivery. Although it improved results with respect the ePID without IFB [176], 3 patients needed supplemental carbohydrates in order to avoid hypoglycemia.

More recently, a modular control-to-range strategy [118] which adds a safety supervision module to avoid hypoglycemia has been clinically tested [23]. This safety module uses IOB constraints implemented in a MPC controller [52] and "power brakes" [75] that attenuate the basal rate to determine the safe insulin dose. The trial involved 11 adolescents and 27 adults and included also a premeal bolus. It tested the performance of two control-torange algorithms, one more conservative and another with a tightest target range, versus CSII open-loop therapy. Although both strategies improved the time in normoglycemia with respect to open-loop (from 61 to $74 \%$ in one case and from 76 to $90 \%$ in the other), some hypoglycemia events were still found after exercise and in the late postprandial period. 
Finally, the use of counterregulatory actions (glucagon) to avoid hypoglycemias is also being investigated [29, 47]. In [47] MPC was used for insulin delivery and a PID for glucagon delivery. Glucose was measured intravenously. The trial reported that 5 from 11 adults being controlled required additional carbohydrates to avoid hyperglycemia due to slower insulin absorption. A later study found that the effectiveness of glugagon was inversely related to the error in the controller insulin level estimations and the magnitude of IOB [144]. The third-generation of the algorithm has been recently tested using CGMS both in inpatient [46] and outpatient conditions [145]. In this last work two 5-days studies, one with 20 adults and with 32 adolescents with different controlled outpatient condition are presented. In both cases the percentage of time in the desired range (here $70-180 \mathrm{mg} / \mathrm{dl}$ ) increased during days $2-5$ of closed-loop therapy ( 79.5 vs $58.8 \%, \mathrm{p}<0.001$ in adults and $75.9 \%$ vs $64.5 \%, \mathrm{p}<0.001$ in adolescents) that was accompanied by a decrease in percentage of time below $70 \mathrm{mg} / \mathrm{dl}$ in the case of the adult cohort $(4.1 \%$ vs. $7.3 \%, \mathrm{p}=0.01)$. The main difficulty that these approaches found is the lack of an FDA-approved stable glucagon preparations that can be infused via subcutaneous pump [133].

\section{Future directions}

As it has been reviewed, overnight closed glucose control has already been validated with good results in controlled environments. Nowadays, algorithms are being tested in more realistic situations. In that way, the DREAM group recently published data from a multinational camp two nights study in 56 children [124 testing MD-logic algorithm. The number of overnight hypoglycemic events was reduced in the closed-loop scheme (7 vs 22). The algorithm is also being tested in adults under domiciliary conditions. Interim results were published in [112]. Cambridge group and AP@home consortium have also reported preliminary promising results of wearable closedloop systems in outpatient studies [50, 36]. More recently, in [72] a 3-weeks outpatient study in adolescent is presented. Nights with glucose $<63 \mathrm{mg} / \mathrm{dl}$ for at least $20 \mathrm{~min}$ were reduced from 17 to $10 \%(\mathrm{p}=0.01)$. Several other wearable systems are currently being tested in clinical trials.

With relation to postprandial control, several trials are currently being 
carried out to improve the counteraction of the meals and to increase safety and robustness against variability and the delay in insulin action. A recent study in supervised transitional outpatient conditions and using the control-torange algorithm implemented in a smartphone (DiAs [89]) showed a reduction in hypoglycemia events per patient from 2.39 to $1.22(\mathrm{p}=0.02)$. However the the percentage of time in the target range was slightly lower. This and other algorithms are currently being tested both in inpatient and outpatient settings. Additionally, strategies combining insulin closed-loop system with drugs already used used in DM2 such as pramlintine and liraglutide are also being tested [175].

In Table 2.2, the most relevant clinical studies that are currently ongoing are listed. Results presented in chapter 4 are currently being tested clinically in study NCT02100488.

\subsection{Conclusions}

Current strategies for glucose control were explained in detail in this chapter. The main insulin therapies used in clinical practice were reviewed paying special attention to intensive insulin therapies and to the advances that are being implemented in CSII pumps. Closed-loop strategies that try to improve glucose control and quality of life of DM1 patients were carefully explained. The characteristics of the measuring and the infusing devices were listed so as to understand their possibilities and limitations.

The purpose of this review was to understand what are the challenges of glucose control and the difficulties that have not been solved yet. Meals counteraction avoiding late hypoglycemia is still an unsolved problem both in open and closed-loop strategies, mainly due to the delays in subcutaneous insulin action and to the high inter and intra-patient variability. On one hand, in open-loop therapies, despite the existence of bolus advisors and the possibilities of infusing smart bolus waveforms in new insulin pumps, the selection of the postprandial doses is still an empirical process based on the experience of patient and physician. On the other hand, in order to 
Table 2.2: Current clinical studies validating closed-loop control systems

\begin{tabular}{|c|c|c|c|}
\hline & SPONSOR/COLLABORATORS & NCT NUMBER & TITLE \\
\hline \multirow{17}{*}{ 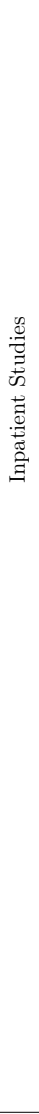 } & \multirow[t]{2}{*}{ Rabin Medical Center } & NCT01901913 & $\begin{array}{l}\text { MD-Logic Artificial Pancreas for Automatic Type } 1 \text { Diabetes } \\
\text { Meals Management }\end{array}$ \\
\hline & & NCT00541515 & $\begin{array}{l}\text { Closing the Loop Between Glucose Sensor and Insulin Pump- } \\
\text { developing an Algorithm }\end{array}$ \\
\hline & \multirow[t]{2}{*}{$\begin{array}{l}\text { Institut de Recherches Cliniques de } \\
\text { Montreal }\end{array}$} & NCT02282254 & $\begin{array}{l}\text { Closed-loop Control of Overnight Glucose Levels (Artificial } \\
\text { Pancreas) in Type } 1 \text { Diabetes Adults With Hypoglycemia } \\
\text { Unawareness and Documented Nocturnal Hypoglycemia }\end{array}$ \\
\hline & & NCT01930110 & $\begin{array}{l}\text { Closed-loop Control of Glucose Levels (Artificial Pancreas) } \\
\text { During Exercise in Adults With Type } 1 \text { Diabetes }\end{array}$ \\
\hline & \multirow{4}{*}{ Yale University } & NCT01787318 & $\begin{array}{l}\text { Effect of the InsuPatch on Automated Closed-loop Glucose } \\
\text { Control in Type } 1 \text { Diabetes }\end{array}$ \\
\hline & & NCT02135068 & $\begin{array}{l}\text { Preventing Hypoglycemia During Exercise With Proactive } \\
\text { Snacking on Closed Loop }\end{array}$ \\
\hline & & NCT01945099 & $\begin{array}{l}\text { Acceleration of Insulin Action by Hyaluronidase During } \\
\text { Closed-Loop Therapy }\end{array}$ \\
\hline & & NCT01856790 & $\begin{array}{l}\text { Effect of Liraglutide on Automated Closed-loop Glucose } \\
\text { Control in Type } 1 \text { Diabetes }\end{array}$ \\
\hline & University of Cambridge & NCT02129868 & $\begin{array}{l}\text { Automated Closed-loop in Children and Adolescents With } \\
\text { Type } 1 \text { Diabetes }\end{array}$ \\
\hline & Medtronic Diabetes & NCT01712594 & $\begin{array}{l}\text { Safety and Effectiveness Study of a Closed Loop System } \\
\text { Maintaining Patients' Glucose Levels During an Overnight } \\
\text { Period }\end{array}$ \\
\hline & $\begin{array}{l}\text { Albert Einstein College of Medicine of } \\
\text { Yeshiva University }\end{array}$ & NCT01755416 & $\begin{array}{l}\text { Liraglutide Versus Insulin Mono-therapy in the Closed Loop } \\
\text { Setting }\end{array}$ \\
\hline & $\begin{array}{l}\text { Fundación para la Investigación del Hos- } \\
\text { pital Clínico de Valencia }\end{array}$ & NCT02100488 & $\begin{array}{l}\text { Improving Postprandial Glycaemia by a New Developed } \\
\text { Closed-loop Control System - Closedloop4meals }\end{array}$ \\
\hline & University of Virginia & NCT01945060 & $\begin{array}{l}\text { Closed Loop Control in Adolescents Using Heart Rate as } \\
\text { Exercise Indicator }\end{array}$ \\
\hline & $\begin{array}{l}\text { Azienda Ospedaliera Universitaria Integ- } \\
\text { rata Verona }\end{array}$ & NCT02003274 & $\begin{array}{l}\text { Mixed Meal Test in Type } 1 \text { Diabetes on Insulin Pump } \\
\text { Therapy: Optimization of Artificial Pancreas }\end{array}$ \\
\hline & Joslin Diabetes Center & NCT02065895 & Effect of Gain on Closed-Loop Insulin \\
\hline & Hvidovre University Hospital & NCT02232971 & $\begin{array}{l}\text { Treatment of Low Blood Sugar With Glucagon Among } \\
\text { Patients With Type } 1 \text { Diabetes }\end{array}$ \\
\hline & $\begin{array}{l}\text { Centre d'Etudes et de Recherche pour } \\
\text { l'Intensification du Traitement du Diabète }\end{array}$ & NCT02101229 & $\begin{array}{l}\text { Validation of the Artificial Pancreas Diabeloop Algorithm in } \\
\text { the Hospital }\end{array}$ \\
\hline \multirow{11}{*}{ 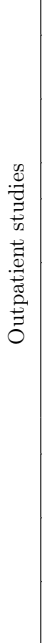 } & Rabin Medical Center & NCT01238406 & Overnight MD-Logic \\
\hline & $\begin{array}{l}\text { Institut de Recherches Cliniques de } \\
\text { Montreal }\end{array}$ & NCT01966393 & $\begin{array}{l}\text { Closed-loop Control of Glucose Levels (Artificial Pancreas) } \\
\text { for } 60 \text { Hours in Adults With Type } 1 \text { Diabetes }\end{array}$ \\
\hline & University of Cambridge & NCT01961622 & $\begin{array}{l}\text { Closing the Loop in Adults With Sub-optimally Controlled } \\
\text { Type } 1 \text { Diabetes Under Free Living Conditions }\end{array}$ \\
\hline & Medtronic Diabetes & NCT01857973 & Overnight Closed Loop Study in U.S. \\
\hline & St Vincent's Hospital Melbourne & NCT02040571 & $\begin{array}{l}\text { The Performance of an Artificial Pancreas at Home in People } \\
\text { With Type } 1 \text { Diabetes }\end{array}$ \\
\hline & \multirow{3}{*}{ University of Virginia } & NCT01890954 & $\begin{array}{l}\text { Optimizing Closed-Loop Control of Type } 1 \text { Diabetes Mellitus } \\
\text { in Adolescents }\end{array}$ \\
\hline & & NCT02302963 & $\begin{array}{l}\text { Unified Safety System (USS) Virginia Closed-Loop Versus } \\
\text { Sensor Augmented Pump Therapy for Hypoglycemia } \\
\text { Reduction in Type } 1 \text { Diabetes }\end{array}$ \\
\hline & & NCT02147860 & Full Day and Night Closed-Loop With DiAs Platform \\
\hline & $\begin{array}{l}\text { Juvenile Diabetes Research Foundation } \\
\text { Artificial Pancreas Project Consortium }\end{array}$ & NCT02137512 & $\begin{array}{l}\text { Pilot Study } 3 \text { of Outpatient Control-to-Range: Safety and } \\
\text { Efficacy With Day-and-Night In-Home Use } \\
\end{array}$ \\
\hline & Stanford University & NCT02280863 & $\begin{array}{l}\text { Hybrid Closed-Loop Hotel Studies With Medtronic PID } \\
\text { Controller }\end{array}$ \\
\hline & $\begin{array}{l}\text { Massachusetts General Hospital |Boston } \\
\text { University }\end{array}$ & NCT02181127 & $\begin{array}{l}\text { Closed-Loop Glucagon Administration For Hypoglycemia } \\
\text { Treatment }\end{array}$ \\
\hline
\end{tabular}
http://clinicaltrials.gov [closed-loop and type 1 Diabetes] 
make the future artificial pancreas feasible, more advancements have to be implemented so as to improve safety against controller overcorrection that lead to hypoglycemia.

Next chapters deal with the main proposals of this thesis to improve glucose control in the postprandial period adding robustness to current strategies. In that way, in next chapter an open-loop proposal to select the best basal-bolus concentration to counteract a specific meal is presented. 


\section{Open-loop strategy: Com- bining basal-bolus insulin in- fusion for tight postpandial glucose control}

In Chapter 2 the limitations and challenges of current insulin therapies have been presented. Despite the recent development of CGMS and smart insulin pumps and the advances in new insulin analogs, postprandial glucose control is still a challenging issue in everyday diabetes care. Insulin dosing remains as an empirical process, and its success is highly dependent on the patients' and physicians' skills, either with multiple daily injections or with CSII.

In this chapter, an algorithm based on mathematically guaranteed techniques (interval analysis) to calculate, in a non-heuristic way, the best prandial basal-bolus combination leading to a tight postprandial glucose control is presented.

Section 3.2 reviews the main theoretical concepts related to interval analysis and set inversion, presenting the set inversion via interval analysis (SIVIA) algorithm and the definitions needed to understand it. The new methodology for prandial insulin determination is introduced in Section 3.3 . Section 3.4 presents the characteristics of the in silico validation whereas a proof-ofconcept clinical study is presented in Section 3.5 . 
Part of the contents of this chapter appeared in the following journal articles:

- A. Revert, P. Rossetti, R. Calm. J. Vehí, J. Bondia, Combining BasalBolus Insulin Infusion for Tight Postprandial Glucose Control. An In Silico Evaluation in Adults, Children and Adolescents, Journal of Diabetes Science and Technology,(6), 1424-1437, 2010 [138]

- A. Revert, R. Calm, J. Vehí, J. Bondia, Calculation of the best basalbolus combination for postprandial glucose control in insulin pump therapy, IEEE Transactions on Biomedical Engineering,58(2), 274-281, 2011 [136]

- P. Rossetti, F.J. Ampudia-Blasco, A. Laguna, A. Revert, J. Vehí, J.F. Ascaso, J. Bondia, Evaluation of a novel continuous glucose monitoringbased method for mealtime insulin dosing - the iBolus - in subjects with type 1 diabetes using continuous subcutaneous insulin infusion therapy: a randomized controlled trial, Diabetes Technology and Therapeutics, 14(11), 1043-1052, 2012 [140

\subsection{Introduction}

Achieving good postprandial glycemic control, without triggering hypoglycemia events, is a challenge of treatment strategies for type 1 diabetes subjects. Continuous subcutaneous insulin infusion, the gold standard of therapy, is based on heuristic adjustments of both basal and prandial insulin. Some tools, such as bolus calculators, are available to aid patients in selecting a mealrelated insulin dose. However, they are still based on empiric parameters such as the insulin-to-carbohydrate ratio and on the physicians' and patients' ability to fit bolus mode to meal composition.

The main challenge that still needs to be overcome is the high intra-patient variability which difficulties the achievement of good glucose control. This is particularly notable in the control of postprandial glycemia excursions. 
Open-loop Proposal: Combining basal-bolus insulin infusion for tight postpandial glucose control

Certainly, meals are one of the major perturbations to counteract and avoidance of overcorrection and subsequent hypoglycemia one of the objective of current therapies.

Interval techniques [78, 107, 6] have shown to be particularly suitable to deal with constraints under uncertainty, and they are applied in a wide range of fields such as robotics, control, computer graphics, economy, global optimization, and fault detection, among others [131. These techniques were first introduced in the context of postprandial glucose control in insulin pump therapy for T1DM in [20].

In this work, the ideas of [20] are used to posed a 3D (basal, bolus, time of restoration of basal to baseline) Set Inversion problem to determine, for a given meal, which prandial insulin administration mode and dose will yield a good postprandial performance (following the International Diabetes Federation (IDF) guideliness [77]), according to the patient's model. The solution can lead to standard, square, and dual-wave boluses modes currently available in insulin pumps, as well as temporal basal decrement mode (currently not available in pumps), which can be considered as a generalization of the concept of superbolus introduced by Walsh and Roberts [171, 170].

A unique feature of this algorithm is that it allows the addition on uncertainty in the parameters of the patient's models and also in the estimated carbohydrate content of the meals. This fact makes this algorithm more robust to variability than those currently implemented in insulin pumps. The proposal was validated in silico using the available patients of the FDA-approved UVa simulator (described in Section 1.4.2) with the consideration of structural mismatch between the patient's model and the model used to describe he virtual patient. Afterwards, the algorithm was also tested in a controlled inpatient clinical study to validate this prandial insulin dosing algorithm in comparison with traditional I:C ratio-based therapies in T1DM subjects using CSII. 


\subsection{Theoretical framework: Interval Analisis and Set Inversion}

Interval analysis arose in the context of numerical analysis and the study of propagation of computational errors in finite number systems [107, 131]: if real numbers are substituted by compact subsets of the digital scale (intervals) which contain it, and real operators by interval operators, computations will lead to intervals that contain the actual solution, whose width is a measure of the approximation error. It is precisely this property of inclusion of the actual solution that makes interval analysis and methods derived very interesting when a mathematical guarantee is desired.

Inclusion functions are thus one of the fundamental tools in interval analysis.

In the following $[x]$ will denote a real interval, and $\underline{x}, \bar{x}$ are its left and right endpoints. Interval vectors, or boxes, will be denoted in boldface, $[\mathbf{x}]$. The set of all real intervals will be denoted by $\mathbb{I R}$ and the set of $n$-dimensional boxes as $\mathbb{R}^{n}$.

A formal definition follows.

Definition 3.2.1 Given a function $\mathbf{f}: \mathbb{R}^{n} \rightarrow \mathbb{R}^{m}$, the interval function $[\mathbf{f}]$ : $\mathbb{I}^{n} \rightarrow \mathbb{I}^{m}$ is an inclusion function for $\mathbf{f}$ if for any box $[\mathbf{x}]=[\underline{\mathbf{x}}, \overline{\mathbf{x}}] \in \mathbb{I}^{n}$

$$
[\mathbf{f}]([\mathbf{x}]) \supseteq\left[\min _{\mathbf{x} \in[\mathbf{x}]} \mathbf{f}(\mathbf{x}), \max _{\mathbf{x} \in[\mathbf{x}]} \mathbf{f}(\mathbf{x})\right] .
$$

The simplest way to get an inclusion function for $\mathbf{f}$ is replacing the real variable $x$ with an interval variable $[x]$ and the real arithmetic operations with corresponding interval operations. The result $[\mathbf{f}]$ is called a natural inclusion function of $\mathbf{f}$ [107]. However, this may yield significant overestimation when multiple instances of a variable appear in the expression to evaluate (multi incidences problem). Other inclusion functions have been studied to reduce this problem like centered forms or Taylor expansion forms. See for instance [6, 131, 107, 78, for more details on this topic. 
Open-loop Proposal: Combining basal-bolus insulin infusion for tight postpandial glucose control

Currently, interval analysis is a mature technology that has been successfully applied in fields aside numerical analysis such as robotics, control, computer graphics, economy, global optimization, and fault detection, among others [78].

An important application of interval analysis is the solution of set inversion problems. Let $\mathbb{X} \subseteq \mathbb{R}^{n}$ and $\mathbb{Y} \subseteq \mathbb{R}^{m}$ be an input and output space, respectively. Given a set $\mathcal{Y} \subseteq \mathbb{Y}$ and a map $\mathbf{f}: \mathbb{X} \rightarrow \mathbb{Y}$, the set $\mathcal{X}:=\{\mathbf{x} \in \mathbb{X} \mid \mathbf{f}(\mathbf{x}) \in \mathcal{Y}\}$ is sought. The set $\mathcal{Y}$ is usually defined through constraints on the output space. The SIVIA algorithm [78 makes use of a branch-and-bound technique together with interval analysis to get an approximation of the solution set $\mathcal{X}$. This approximation is done in terms of subpavings (collection of boxes of the appropriate dimension with non-overlapping interiors). An inner and outer subpaving, which will be denoted as $[\mathcal{X}]_{\mathrm{i}}$ and $[\mathcal{X}]_{\mathrm{o}}$ respectively, are built so that $[\mathcal{X}]_{\mathrm{i}} \subseteq \mathcal{X} \subseteq[\mathcal{X}]_{\mathrm{o}}$. Hence, it is guaranteed that $[\mathcal{X}]_{\mathrm{i}}$ will contain only solutions while the complementary set of $[\mathcal{X}]_{\mathrm{o}}$, denoted as $\overline{[\mathcal{X}]_{\mathrm{o}}}$, will contain only non-solutions (see Figure 3.1.

Some previous definitions follow before presenting the SIVIA algorithm.

Definition 3.2.2 The width of a box $[\mathbf{x}]=[\underline{\mathbf{x}}, \overline{\mathbf{x}}] \in \mathbb{R}^{n}$ is $w([\mathbf{x}]):=$ $\max _{i \in\{1, \ldots, n\}}\left(\overline{x_{i}}-\underline{x_{i}}\right)$.

Definition 3.2.3 The midpoint of a box $[\mathbf{x}]=[\underline{\mathbf{x}}, \overline{\mathbf{x}}] \in \mathbb{I}^{n}$ is $m([\mathbf{x}]):=(\underline{\mathbf{x}}+$ $\overline{\mathbf{x}}) / 2$.

Definition 3.2.4 The left and right children of a box $[\mathbf{x}]=[\underline{\mathbf{x}}, \overline{\mathbf{x}}] \in \mathbb{R}^{n}$ are

$$
\begin{aligned}
L([\mathbf{x}]) & :=\left[\underline{x_{1}}, \overline{x_{1}}\right] \times \cdots \times\left[\underline{x_{j}}, m\left(\left[x_{j}\right]\right)\right] \times \cdots \times\left[\underline{x_{n}}, \overline{x_{n}}\right] \\
R([\mathbf{x}]) & :=\left[\underline{x_{1}}, \overline{x_{1}}\right] \times \cdots \times\left[m\left(\left[x_{j}\right]\right), \overline{x_{j}}\right] \times \cdots \times\left[\underline{x_{n}}, \overline{x_{n}}\right]
\end{aligned}
$$

where $j$ is the first component of $[\mathbf{x}]$ with maximum width, that is, $j=$ $\min \left\{i \mid w\left(\left[x_{i}\right]\right)=w([\mathbf{x}])\right\}$.

Algorithm 3.2.1 [SIVIA, [78]] 


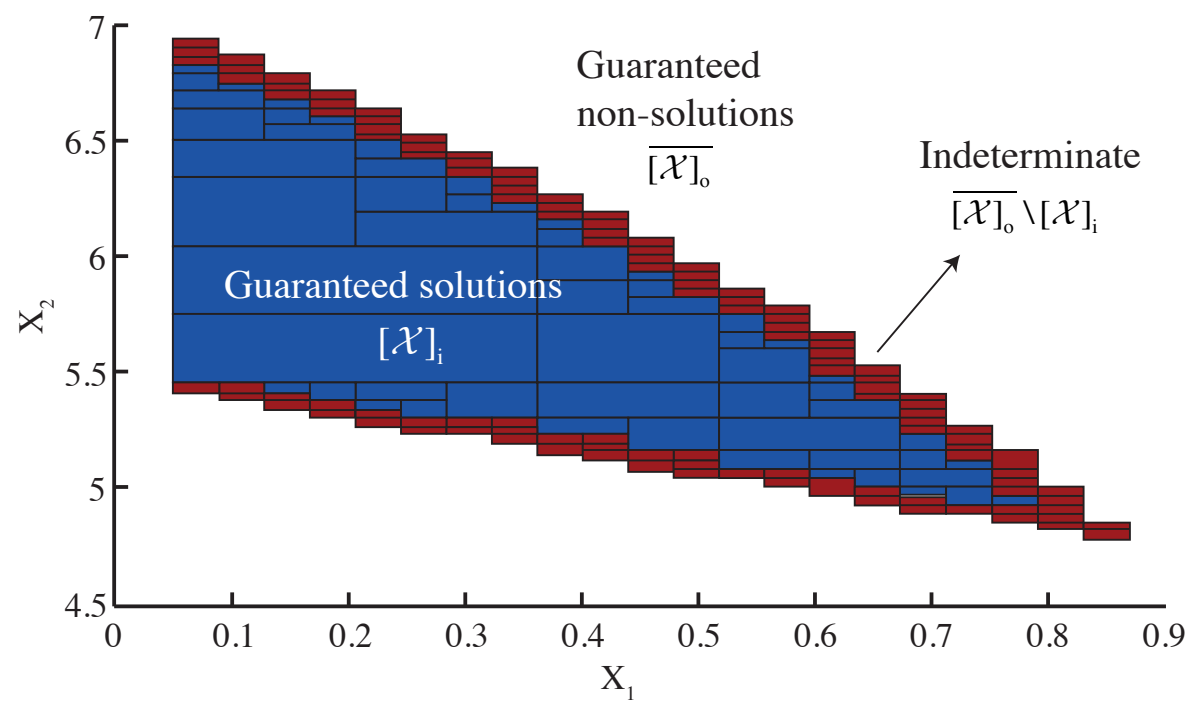

Figure 3.1: Plot that illustrates the concept of inner and outer subpaving. Blue rectangles represent the inner subpaving and guarantee the fulfillment of the constraints. The outer subpaving is made up of both blue and red rectangles. Its complementary set (in white) is guaranteed to contain only non-solutions that violate some of the constraints. Results in the boundary (red rectangles) are unknown a priori. 
Open-loop Proposal: Combining basal-bolus insulin infusion for tight postpandial glucose control

Let $\mathcal{X}$ be the solution set sought and $[\mathcal{X}]_{\mathrm{i}}$ and $[\mathcal{X}]_{\mathrm{o}}$ be two subpavings corresponding to inner and outer approximations of $\mathcal{X}$ as defined above. Let $[t]: \mathbb{R}^{n} \rightarrow \mathbb{I B}$ be a test interval function from the set of $n$-dimensional interval vectors (box in the input space) to the set of interval booleans, $\mathbb{I} \mathbb{B}=\{0,1,[0,1]\}$ (where 0 stands for false, 1 for true and $[0,1]$ for indeterminate). Finally, let $[\mathbf{x}] \in \mathbb{R}^{n}$ be an initial box in the input space and $\epsilon$ be a positive precision factor that can be chosen arbitrarily low. The SIVIA algorithm is as follows:

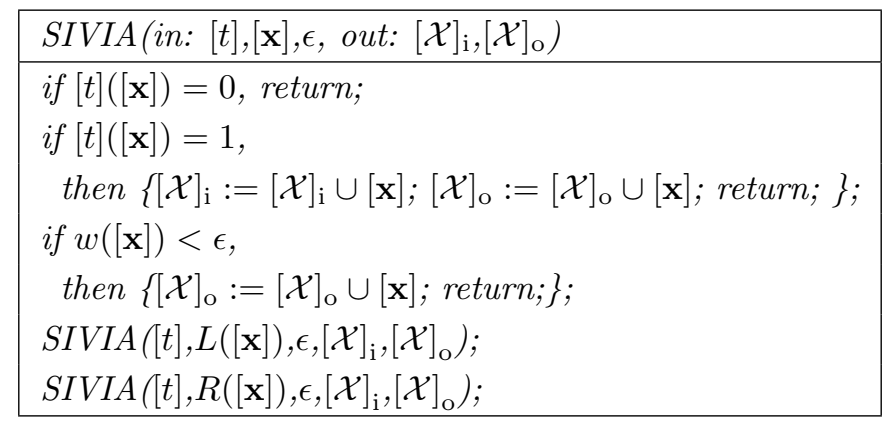

The inner subpaving will thus consist of the boxes classified as True, while the outer subpaving contains the True and Indeterminate boxes (of width smaller than the tolerance defined). Not small enough Indeterminate boxes will be split in two sub-boxes by the midpoint of its largest dimension and the procedure repeated.

\subsection{Set-Inversion-Based prandial insulin delivery}

With a proper instantiation of the input and output spaces, $\mathbb{X}$ and $\mathbb{Y}$, and the test interval function [t], SIVIA algorithm can be used to gain insight on the different dosage strategies that can be applied depending on the patient and the nature of the meal and to select the best basal-bolus combination that will yield a good postprandial control.

For this purpose, the following set inversion problem is posed:

- The input space $\mathbb{X}$ corresponds to the bolus insulin dose, the modified basal insulin infusion at meal time and the time of restoration of basal 
to its baseline value. For a given box in the input space, $[\mathbf{x}]$, and a set of constraints $\mathcal{C}$ on postprandial glycemia, the test function $[t]([\mathbf{x}])$ will determine whether: (1) all the insulin therapies contained in $[\mathbf{x}]$ fulfill the constraints $\mathcal{C}$ (True case); (2) none of the insulin therapies contained in $[\mathbf{x}]$ fulfill the constraints $\mathcal{C}$ (False case); (3) some of the therapies in $[\mathbf{x}]$ fulfill the constraints, while others do not (Indeterminate case).

- The constraints $\mathcal{C}$ are defined as follows:

1. Constraints based on the IDF guidelines for postmeal control [77]: non-hypoglycemia and two-hour postprandial glucose value below $140 \mathrm{mg} / \mathrm{dl}$, in a 5-hour time horizon. The hypoglycemic threshold is not explicitly defined in the guidelines. A value of $70 \mathrm{mg} / \mathrm{dl}$ is adopted here following clinical practice.

2. Terminal constraints: 5-hour postprandial glucose value above 90 $\mathrm{mg} / \mathrm{dl}$ and a maximum glucose slope of $0.05 \mathrm{mg} / \mathrm{dl} / \mathrm{min}$ starting 4 hours after the meal (i.e., conditions of glycemic stability).

Terminal constraints are included here to minimize both the risk of hypoglycemia after the first five hours and late undesirable glucose rebounds. It must be considered that these are constraints applied on the model prediction, which may not be reliable enough after a few hours after the meal. They were tuned so as to get a good patient's behavior in spite of model prediction discrepancies.

- Finally, a patient's model is used to predict postprandial glycemia, $G$, that is compared against the above constraints. An interval simulation of the model is carried out using Modal Interval Analysis [55]. This allows to obtain tight bounds of the envelopes enclosing the collection of postprandial glucose profiles originated from the set of therapies in $[\mathbf{x}]$. For a given time $t,[G](t)$ will thus be an interval (see Figure 3.2). A time step of 1 minute is used in the simulation.

The test interval function, $[t]([\mathbf{x}])$ is thus defined as: 
Open-loop Proposal: Combining basal-bolus insulin infusion for tight postpandial glucose control

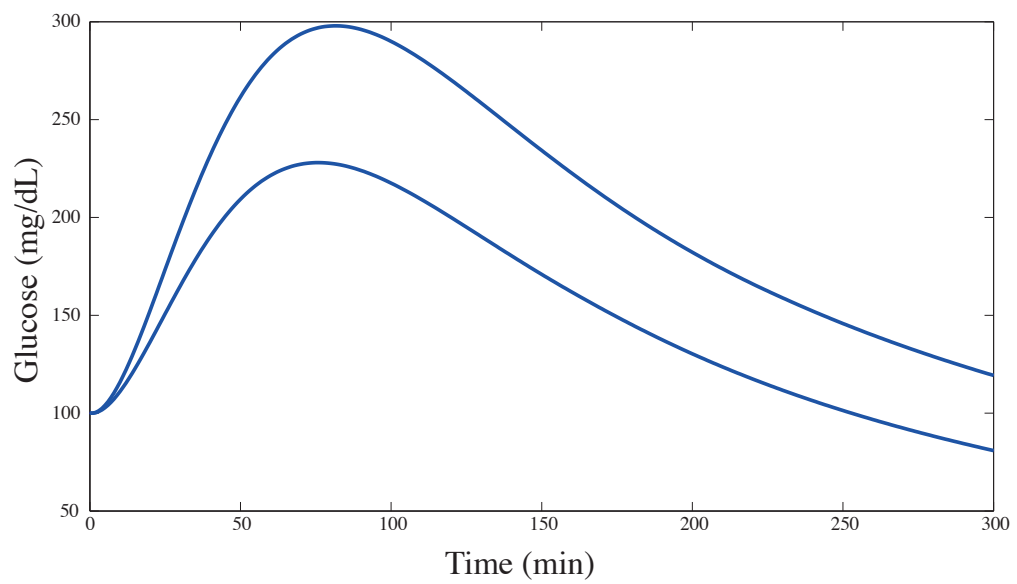

Figure 3.2: Output of an interval simulation. Upper and lower envelopes include all possible glucose responses for the input box.

$$
\begin{array}{cl}
\text { True: } & \left(\forall t_{k} \in[0,300][G]\left(t_{k}\right) \geq 70\right) \wedge \\
& \left(\forall t_{k} \in[120,300][G]\left(t_{k}\right) \leq 140\right) \wedge \\
& [G](300) \geq 90) \wedge \\
& \left(\forall t_{k} \in[240,300] \frac{[G]\left(t_{k+1}\right)-[G]\left(t_{k}\right)}{t_{k+1}-t_{k}} \leq 0.05\right) \\
\text { False: } \quad & \left(\exists t_{k} \in[0,300][G]\left(t_{k}\right)<70\right) \vee \\
& \left(\exists t_{k} \in[120,300][G]\left(t_{k}\right)>140\right) \vee \\
& ([G](300)<90) \vee \\
& \left(\exists t_{k} \in[240,300] \frac{[G]\left(t_{k+1}\right)-[G]\left(t_{k}\right)}{t_{k+1}-t_{k}}>0.05\right)
\end{array}
$$

Indet.: otherwise

where $t_{k}$ is a discrete time instant. Remark that the above inequalities correspond to interval inequalities $([\underline{x}, \bar{x}] \leq \alpha \Leftrightarrow \bar{x} \leq \alpha,[\underline{x}, \bar{x}] \geq \alpha \Leftrightarrow \underline{x} \geq$ $\alpha, \alpha \in \mathbb{R})$.

An schematic representation of the proposal is shown in Figure 3.3. 


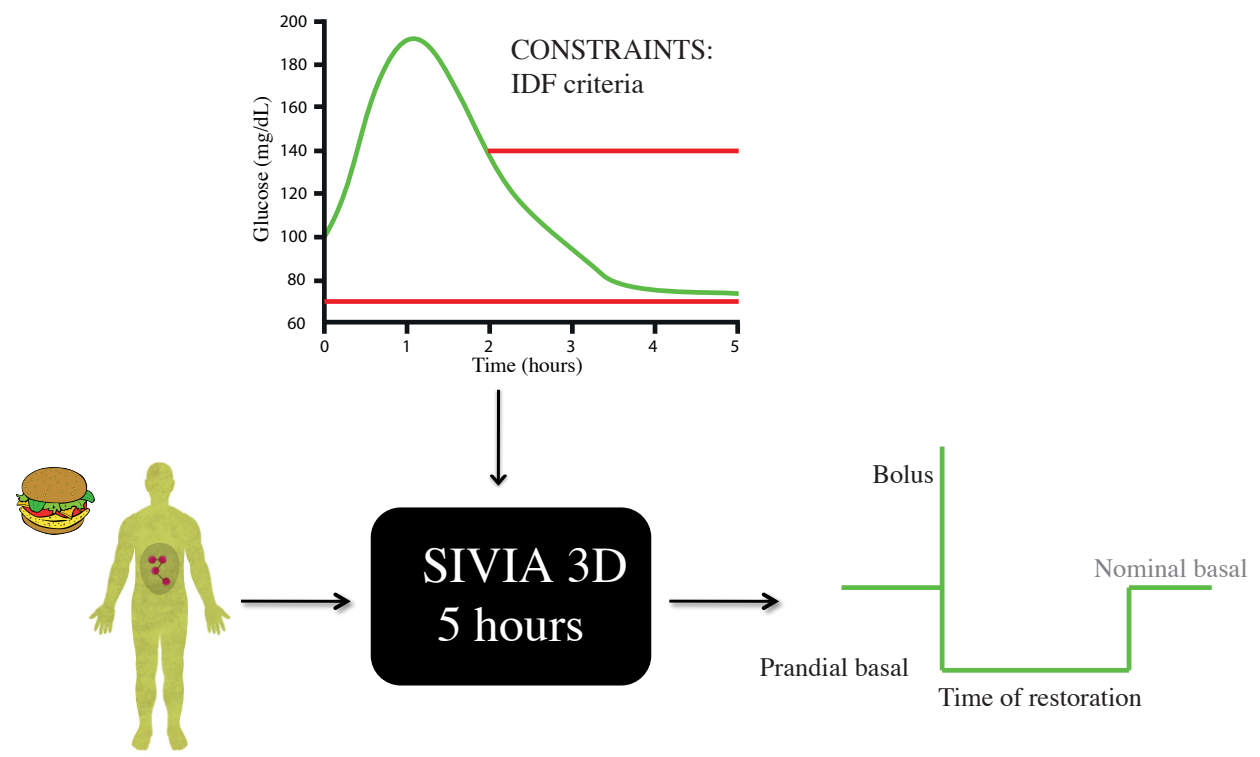

Figure 3.3: Input and outputs of the designed algorithm. By using a mathematical model of the patient and information regarding the meal to be ingested, the SIVIA based algorithm calculates the best basal-bolus insulin dose combination to fulfill a set of constraints according to the IDF criteria of good postprandial control. 
Open-loop Proposal: Combining basal-bolus insulin infusion for tight postpandial glucose control

\subsubsection{Output of the algorithm: best basal-bolus combination}

The kind of inner subpavings that are obtained after the application of the algorithm is shown in Figure 3.4. The subpaving consists of 3D feasible boxes, where these three dimensions correspond to the bolus dose, the postprandial basal dose and time of restoration of basal to baseline. In this way, combinations in the inner subpaving are guaranteed to yield a glucose profile that fulfills the defined constraints. On the contrary, combinations outside the outer subpaving will violate some constraint. If the outer subpaving is empty, there is no solution to the problem unless the constraints are relaxed. To gain interpretability the $3 \mathrm{D}$ set can be projected into the $2 \mathrm{D}$ basal-bolus projection.

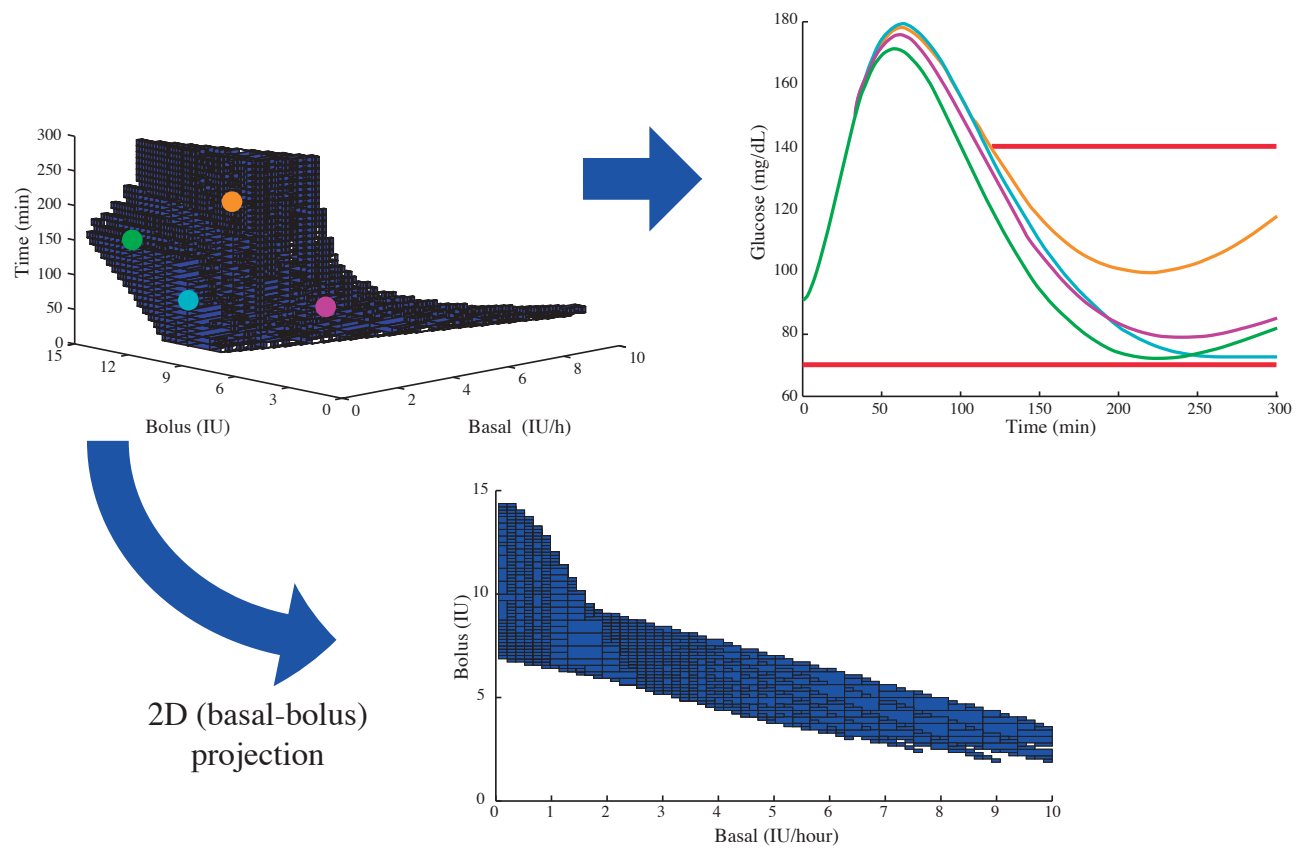

Figure 3.4: Plot that represents a 3D (basal, bolus and time) feasible set with its corresponding basal-bolus $2 \mathrm{D}$ projection.

This 2D projection contains information on the different basal-bolus combinations that will lead to a good performance for a particular patient and meal. The basal-bolus space can be divided into regions corresponding to different bolus administration modes present in current insulin pumps 
(see Figure 2.5 in Chapter 2), plus a new one called here temporal basal decrement.

This is illustrated in Figure 3.5, where basal and bolus axes have been normalized with respect to nominal basal and nominal bolus for the given meal (computed from the patient's I:C), respectively. Point $(1,1)$ corresponds thus to the standard therapy.

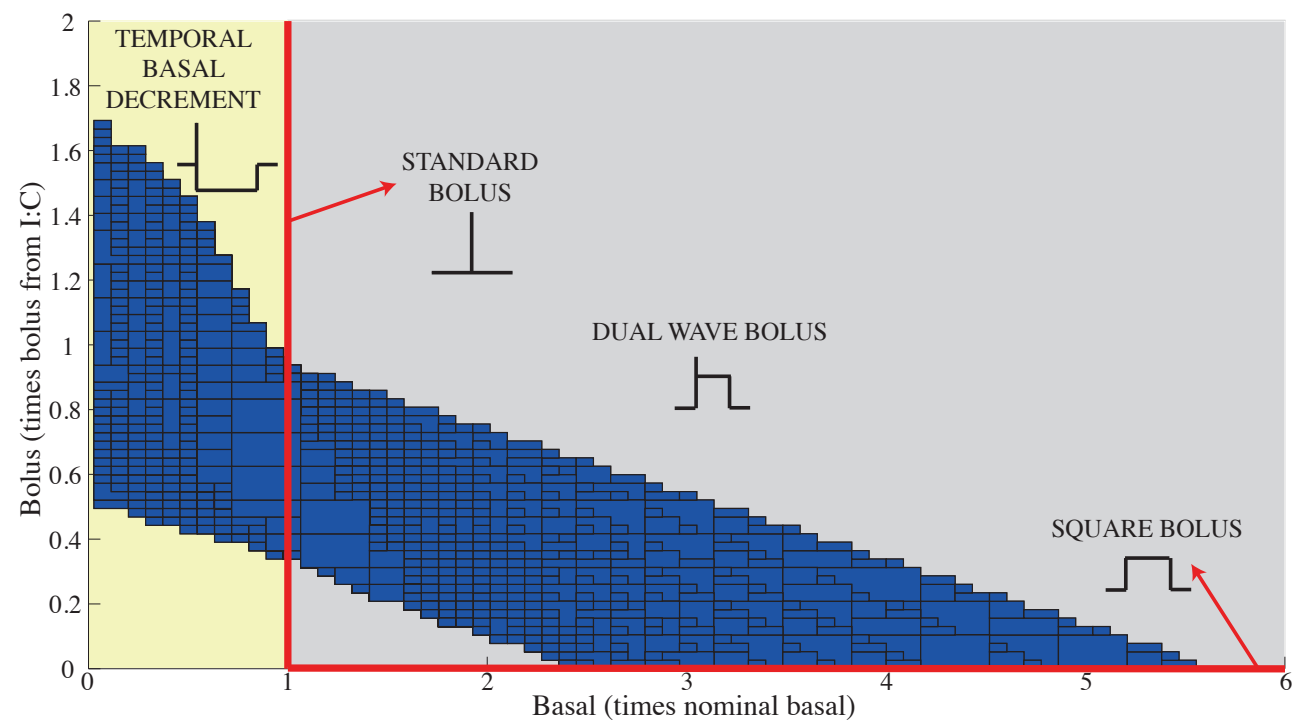

Figure 3.5: Normalized feasible set that shows all the possible bolus administration modes. Therapies with nominal basal correspond to a standard strategy, therapies with an increment in basal postprandial dosage result in a dual-wave or square-wave strategy whereas therapies with less postprandial basal than baseline are called here as temporal basal decrement mode. The corresponding insulin infusion profiles are depicted for each region.

For a given meal, the projected set reveals which bolus administration modes are feasible. This is especially important since it allows the automatic selection of the best administration mode. As the carbohydrate content of the meal increases, fewer options are available, until no solution exists (Figure 3.6). In this case, either the patient reduces the meal intake or constraints must be relaxed.

The procedure used to select a specific point (basal-bolus combination) 
Open-loop Proposal: Combining basal-bolus insulin infusion for tight postpandial glucose control
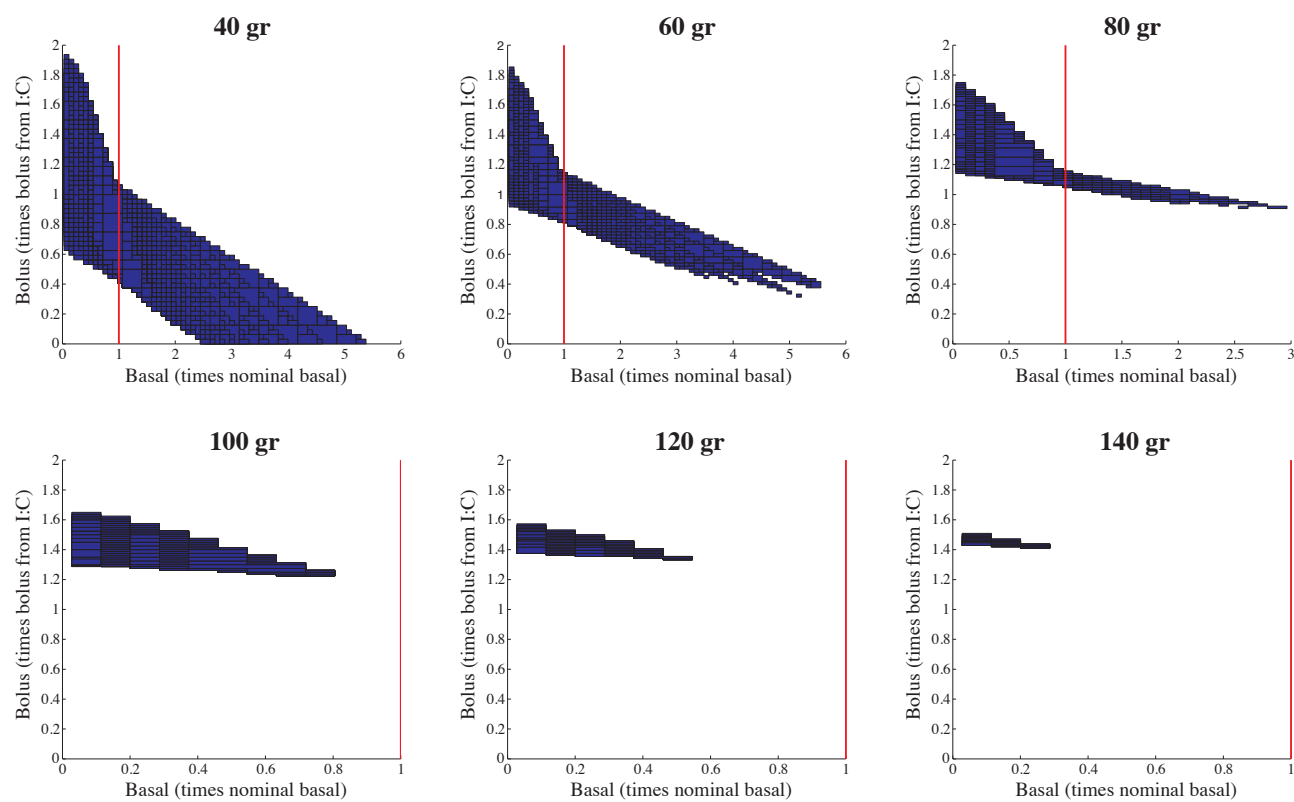

Figure 3.6: 2D basal-bolus projection of normalized feasible sets for a meal of 40 to $140 \mathrm{~g}$ of carbohydrates and initial normoglycemia. The vertical line stands for the standard strategy with basal equal to its baseline value. 
from all the possible ones is done as follows. The 2D basal-bolus subpaving is divided into two smaller subpavings (when possible) corresponding to a positive and negative basal deviation from nominal. The first one corresponds to a bolus mode currently available in insulin pumps (standard/square/dualwave). The second one corresponds to temporal basal decrement, found to be the only solution for big sized meals (see [20]). The optimal point of each of these new subpavings can be selected in several ways, and different approaches will be explained here:

- Centroid solution: The basal-bolus combination is chosen as the geometric centroid of the corresponding subset. This alternative leads to a conservative solution where the glucose response remains as far as possible from the constraints. Although this solution does not optimize the glucose profile, it is the most robust solution against mismatches between patients' model and actual patients.

- Maximal-bolus solution: The basal-bolus combination is chosen by applying the highest possible bolus to optimize the 2-hour postprandial glucose concentration. This solution follows a similar philosophy to the typical physicians' approach for selecting the appropriate I:C ratio for each patient. The difference here is that the coordinated basal-bolus action will allow an optimal 2-hour postprandial glucose control, while avoiding hypoglycemia although loosing robustness.

After the selection of the desired basal-bolus combination, the time of restoration of basal to baseline is selected from the third dimension in the 3D feasible set, which corresponds to an interval of feasible times of restoration. The mid-value is considered here. Figure 3.7 summarizes this procedure.

Summarizing, after identification of the patient's model characterizing postprandial behavior up to 5 hours, the algorithm consists of the following steps:

1. Computation of the feasible set of prandial insulin infusions in the 3D 
Step 1

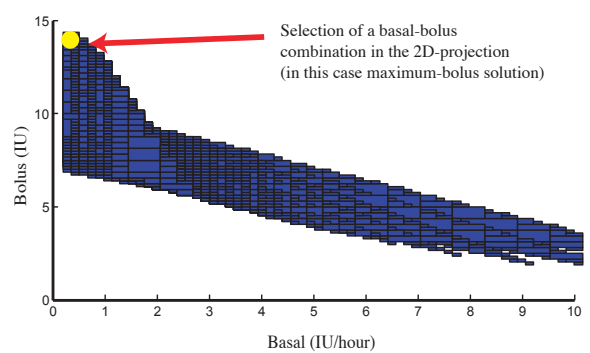

Step 2

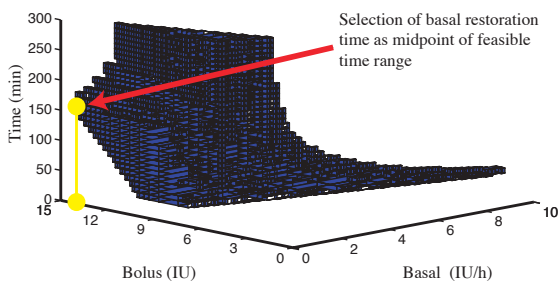

Figure 3.7: Procedure used to select the basal-bolus combination from all the available possibilities.

space (bolus insulin at mealtime, basal rate at mealtime, time of basal restoration).

A. If it is empty, relax constraints and go to 1 .

B. If it is not empty, go to 2 .

2. Projection onto the two-dimensional space (bolus insulin, basal rate at mealtime).

3. Selection of the desired administration mode among the feasible ones in the two-dimensional projection.

4. Selection of a bolus insulin and basal rate at mealtime among the selected subset.

5. Selection of a feasible time of basal restoration, according to the selection in 4 , from the three-dimensional feasible set.

\subsection{In silico evaluation}

Table 3.1 shows the demographic, anthropometric, and metabolic parameters of the 30 patients available in the educational version of UVa simulator. Those patients are used as population set. Nominal basal is taken as the basal infusion 
normalizing glucose around $100 \mathrm{mg} / \mathrm{dl}$, and the I:C ratio is estimated through simulations trying to obtain a 2-h glucose concentration below $140 \mathrm{mg} / \mathrm{dl}$.

Table 3.1: Demographic, anthropometric and metabolic parameters of the 30 in silico subjects available in the educational version of the UVa simulator.

\begin{tabular}{lcccc}
\hline \multicolumn{5}{c}{ ADULTS } \\
\hline & Age & Body Weight (kg) & Nominal basal (IU/h) & I:C $(\mathrm{g} / \mathrm{IU})$ \\
Mean & 51.6 & 86.07 & 1.685 & 9.92 \\
Standard Deviation & 16 & 15.79 & 0.25 & 6.33 \\
\hline \multicolumn{5}{c}{ ADOLESCENTS } \\
\hline Age & Body Weight (kg) & Nominal basal $(\mathrm{IU} / \mathrm{h})$ & $\mathrm{I}: \mathrm{C}(\mathrm{g} / \mathrm{IU})$ \\
Mean & 16.5 & 47.7 & 1.17 & 9.65 \\
Standard Deviation & 1.75 & 7.89 & 0.24 & 6.09 \\
\hline 5cCHILDREN & \multicolumn{5}{c}{} \\
\hline & Age & Body Weight (kg) & Nominal basal $(\mathrm{IU} / \mathrm{h})$ & $\mathrm{I}: \mathrm{C}(\mathrm{g} / \mathrm{IU})$ \\
Mean & 9.4 & 35,865 & 0.502 & 21.11 \\
Standard Deviation & 1.56 & 5.96 & 0.07 & 13.76 \\
\hline
\end{tabular}

The algorithm requires obtaining an individual model for each of the patients, characterizing their postprandial glucose behavior. UVa simulator uses the Cobelli model as a mathematical description of type 1 diabetic patients (see Section 1.4.2). In order to force mismatch between patient's model and the virtual patient behavior for a more realistic scenario, the Hovorka model [74, 71] (Section 1.4.1), which is structurally different, is used here as patient's model.

The use of a model structurally different from the model used in the UVa simulator is justified by the unavoidable discrepancies that always exist between the real behavior of a patient and the response of its model. Choosing a different model for identification than the one used in the simulator allows evaluating the robustness of the algorithm with respect to model and patient mismatch. 


\subsubsection{Patient's model identification}

A critical point of the methodology is the identification of the prediction model of each patient so as to reproduce his metabolism as accurately as possible. A proper design of the procedure used for identification so as to obtain as much information as possible from the patient metabolism is the key to achieve good parameters estimation. Thus, an optimal experiment design (OED) was carried out in order to find the best procedure for the identification.

OED consists in identifying the proper experiment setup (selection of inputs to excite the process) that delivers experimental data allowing parameter estimation with minimal uncertainty. In this manner, OED helps in improving the a posteriori identification of the parameters, minimizing also problems of bad conditioning in their estimation [174, 99].

The specific features of the OED used for patients identification are now described:

Parameters selected for describing the experimental design were:

- ingested carbohydrates,

- bolus insulin dosage, and

- time instant of bolus insulin infusion (before or after the meal).

The duration of each experiment (meal) was set in 5-hours and the number of measurements was fixed and determined by the frequency of the continuous glucose sensor (5 minutes). Each experiment was considered to be carried out on different days, approximately at the same time every day (lunch time).

Bound constraints on the parameters were included to avoid impractical results. So, the grams of carbohydrates were limited to values between 50-100 $\mathrm{g}$ (corresponding to an acceptable range for the lunch) while the bolus insulin dosage was forced to remain in the range $\pm 20 \%$ the patient's standard bolus for that meal. Infusion time for bolus insulin was limited from 30 minutes before the intake to 30 minutes after it. In addition, extra constraints were 
added to avoid hypoglycemia $(<70 \mathrm{mg} / \mathrm{dl})$ or extremely high hyperglycemia $(>300 \mathrm{mg} / \mathrm{dl}$ ).

A priori identifiable parameters reported in [71] were considered for identification, with the exception of $F_{01}$ (non-insulin-dependent glucose flux) that was replaced with $t_{\max , G}$ (time to maximum carbohydrate absorption) so as to include at least one parameter related to the meal intake (see [71] for the model details). Moreover $Q_{2}(0)$ (initial mass of glucose in the non-accessible compartment) was also included as an additional parameter to be identified. The values of the parameters used were mean parameters with the body weight set to the mean body weight of the adults, adolescents and children to be used in the in silico validation. An insulin sensitivity proportionality factor was applied and fixed to 0.5, 0.6 and 0.7 respectively. Nominal basal was taken as the basal infusion normalizing glucose around $100 \mathrm{mg} / \mathrm{dl}$. I:C ratio was estimated through simulations and fixed to 1:14, 1:19 and 1:25 for adults, adolescents and children.

The optimality criterion used was D-optimality, corresponding to the maximization of the determinant of the Fisher Information Matrix [174]. Figure 3.8 shows the evolution of this criterion for the three groups of patients with respect to the number of days (number of lunches) used for identification. It can be observed that the improvement in the criterion resulting from adding a new day decreases progressively. So, a compromise solution between the time needed to identify and the a posteriori identifiability of the model was selected. Therefore, data obtained from four different lunches was used in the identification process.

Table 3.2 shows the OED results obtained for adults, adolescents and children. In general, it can be inferred from the OED that the best results in identification are obtained by alternating the time of administration of the bolus insulin with respect to meal time, always distancing the administration as much as possible from the meal. Moreover, both carbohydrate grams and deviation from nominal I:C must be selected from the extreme values. That is, big or small meals are better for identification than medium size meals. In the same way, the glucose response has to be excited as much as possible delivering 
Open-loop Proposal: Combining basal-bolus insulin infusion for tight postpandial glucose control

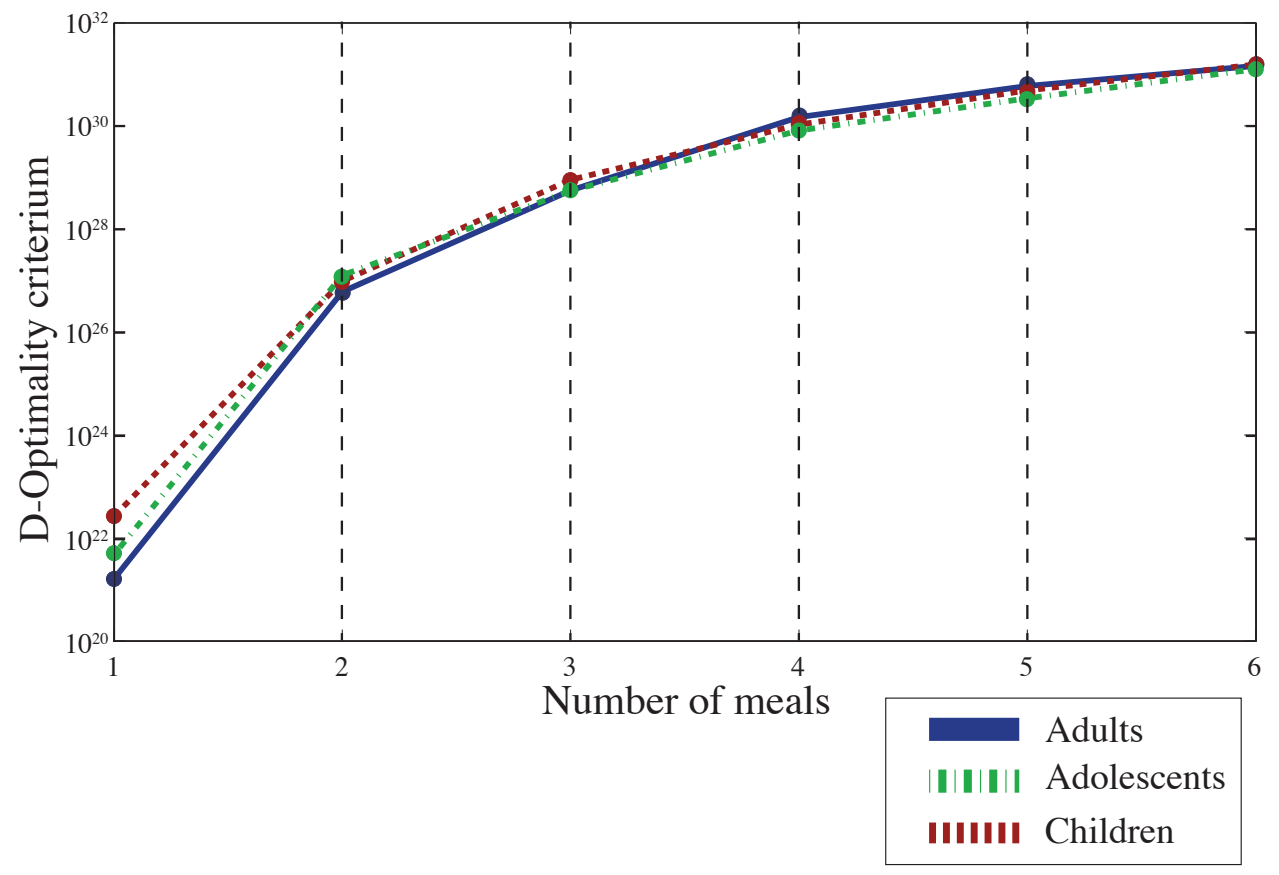

Figure 3.8: Value of the D-optimality criterion against different number of meals for the three groups of patients. 
Table 3.2: Proper four meals experiment setup according to the OED

\begin{tabular}{cccccc}
\hline & & Meal 1 & Meal 2 & Meal 3 & Meal 4 \\
\hline \multirow{4}{*}{ ADULTS } & carbohydrates $(\mathrm{g})$ & 50 & 100 & 50 & 100 \\
& $\triangle(I: C)$ & $20 \%$ & $20 \%$ & $20 \%$ & $-20 \%$ \\
& time $(\min )$ & -30 & -30 & 30 & -30 \\
\hline \multirow{4}{*}{ ADOLESCENTS } & carbohydrates $(\mathrm{g})$ & 100 & 50 & 90 & 100 \\
& $\triangle(I: C)$ & $-20 \%$ & $20 \%$ & $0 \%$ & $20 \%$ \\
& time $(\min )$ & -30 & -30 & 0 & -30 \\
\hline \multirow{3}{*}{ CHILDREN } & carbohydrates(g) & 100 & 70 & 50 & 100 \\
& $\triangle(I: C)$ & $20 \%$ & $17 \%$ & $20 \%$ & $-10 \%$ \\
& time $(\min )$ & -30 & 0 & -30 & -30 \\
\hline
\end{tabular}

bolus the further from nominal the better, but always within control limits to not decompensate the patient in excess. This fact can be clearly observed in the adult group whereas in adolescents and children, due to their higher insulin sensitivity, the possible violation of the bound constraints reduces the freedom in the selection of the experiment parameters.

A posteriori identifiability analysis of the model with the results obtained from Table 3.2 , proved that the seven to-be-identified parameters have a coefficient of variation below $10 \%$, fact that guarantees their identifiability.

A 4-day identification for each of the 30 patients was carried out following the OED results. Results are summarized in Table 3.3 . Figure 3.9 shows the specific identification results obtained for one representative adult patient from the UVa simulator are shown in Figure 3.9. The coefficient of determination, $R^{2}$, is $86 \%$ which assures a good identification quality.

\subsubsection{Metrics used in the in silico evaluation}

Once obtained an individual patient model for each of the 30 virtual patients available, the feasible sets were computed for meals in the range 40-140 
Open-loop Proposal: Combining basal-bolus insulin infusion for tight postpandial glucose control

Meal 1

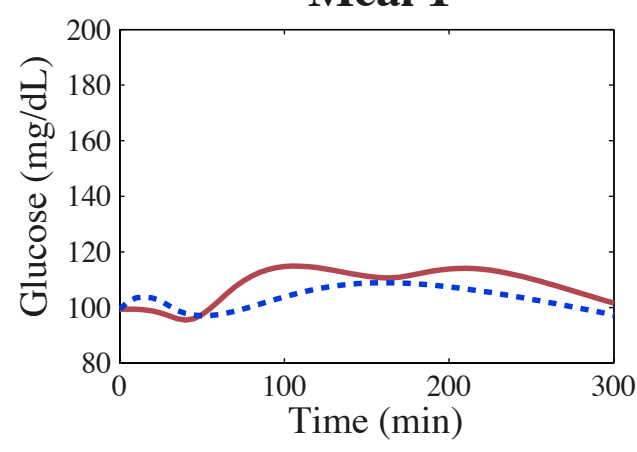

Meal 3

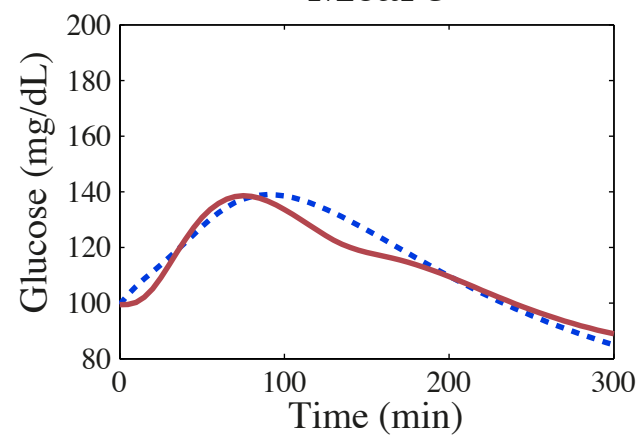

Meal 2

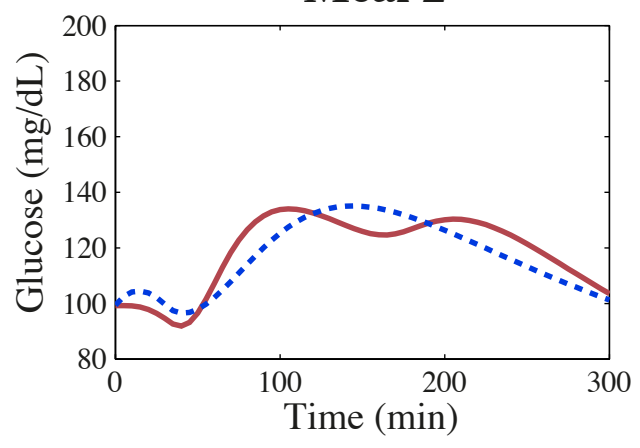

Meal 4

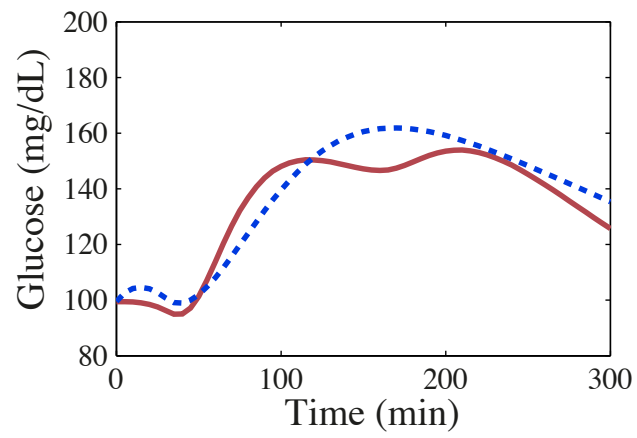

Virtual patient profile

Iu I Simulated profile

Figure 3.9: Four-meal identification following the OED results. The solid red line represents the glucose profile of the virtual patient whereas the dotted blue line represents the profile simulated by the identified model. 
Table 3.3: Model parameters estimation

\begin{tabular}{|c|c|c|c|c|c|c|c|c|}
\hline & & $\begin{array}{r}t_{\max }, I \\
(\min )\end{array}$ & $\begin{array}{c}t_{\max }, G \\
(\min )\end{array}$ & $\begin{array}{c}S_{I T}^{f} \\
(1 / \min \text { per } \mathrm{mIU} / \mathrm{L})\end{array}$ & $\begin{array}{c}S_{I D}^{f} \\
(1 / \text { min } \text { per } \mathrm{mIU} / \mathrm{L})\end{array}$ & $\begin{array}{c}S_{I E}^{f} \\
(1 / \min \text { per } \mathrm{mIU} / \mathrm{L})\end{array}$ & $\begin{array}{c}E G P_{0} \\
(\mathrm{mmol} /(\mathrm{kg} \cdot \mathrm{min}))\end{array}$ & $\begin{array}{l}Q_{2}(0) \\
(\mathrm{mmol})\end{array}$ \\
\hline \multirow{2}{*}{ ADULTS } & Mean & 60.85 & 61.17 & 0.0049 & 0.0034 & 0.0015 & 0.0137 & 47.55 \\
\hline & $\mathrm{CV}$ & 0.27 & 0.23 & 0.98 & 1.13 & 2.20 & 0.35 & 0.37 \\
\hline \multirow{2}{*}{ ADOLESCENTS } & Mean & 53.39 & 59.45 & 0.0045 & 0.00873 & 0.0010 & 0,01590 & 20.84 \\
\hline & $\mathrm{CV}$ & 0.47 & 0.29 & 1.43 & 1.25 & 2.26 & 0.30 & 0.27 \\
\hline \multirow{2}{*}{ CHILDREN } & Mean & 59.19 & 53.07 & 0.0203 & 0.0074 & 0.0160 & 0.0175 & 18.41 \\
\hline & $\mathrm{CV}$ & 0.42 & 0.48 & 1.55 & 0.90 & 1.73 & 0.31 & 0.81 \\
\hline
\end{tabular}

$\mathrm{CV}$ : coefficient of variation

$\mathrm{g}$ of carbohydrates and initial normoglycemia. For this study, the bolus administration mode selected among the feasible sets was the temporal basal decrement. Indeed, for some situations, it is the only feasible solution [20] (see Figure 3.6. Postprandial glucose control efficiency of both the centroid and the maximal-bolus solution of the temporal basal decrement set was measured and compared with the standard bolus. A 7-hour postprandial horizon was considered to evaluate the risk of later hypo and hyperglycemia. Different metrics were used for this comparison:

1. The area under the curve (AUC) for glucose concentrations higher than $140 \mathrm{mg} / \mathrm{dl}$ and lower than $70 \mathrm{mg} / \mathrm{dl}$ was calculated, using the trapezoidal rule [132] in the $0-2 \mathrm{~h}, 0-5 \mathrm{~h}$ and $0-7 \mathrm{~h}$ postprandial periods for the whole 40-140 g range of carbohydrates;

2. percentage of time spent in the desired glycemic range $(70 \mathrm{mg} / \mathrm{dl}<$ glucose $<140 \mathrm{mg} / \mathrm{dl}$ ), as well as percentage of time spent in hypoglycemia (glucose $<70 \mathrm{mg} / \mathrm{dl}$ ), were computed;

3. The blood glucose standard deviation within the $0-5 \mathrm{~h}$ and $0-7 \mathrm{~h}$ time intervals $\left(S D_{w s h}\right)$ [139] was calculated as a measure of the glycemic variability associated with each prandial insulin administration strategy.

Total and bolus insulin dose were also reported, to allow for correct interpretation of the above-mentioned indicators. The $A U C_{0-2 h}, A U C_{0-5 h}$ and the $A U C_{0-7 h}$, as well as the insulin dose, were normalized by the respective values obtained using standard bolus. 
Open-loop Proposal: Combining basal-bolus insulin infusion for tight postpandial glucose control

All data are subjected to repeated-measures analysis of variance with Huynh-Feldt adjustment for nonsphericity [180]. The analysis of variance model included only the test condition (standard bolus, centroid and maximalbolus temporal basal decrement), as within-subjects factor, and post hoc comparisons (Tukey test) were carried out to pinpoint specific differences on significant interaction terms.

\subsubsection{Results of the in silico evaluation}

Tables 3.4, 3.5 and 3.6 show the mean AUC of the three groups of patients (adults, adolescents and children) following meals with different carbohydrate content. A comparison among the percentages of time in normoglycemia (70 $\mathrm{mg} / \mathrm{dl}<$ glucose $<140 \mathrm{mg} / \mathrm{dl}$ ) and hypoglycemia (glucose $\leq 70 \mathrm{mg} / \mathrm{dl}$ ) for each of the solutions is also provided along with the amount of prandial bolus insulin and total insulin dose in the 7 hours postprandial period. One virtual child (child number 8 with $\mathrm{BW}=23.73 \mathrm{~kg}$ ) was eliminated from the study because the feasible set of prandial insulin infusions was empty for meals higher than $60 \mathrm{~g}$ even relaxing constraints.

As a whole, results demonstrate the feasibility and effectiveness of the proposed algorithm based insulin administration. It performed generally better than the traditional bolus in all of the considered time horizons. In particular, both centroid and maximal-bolus temporal basal decrement solutions were associated with significant less hypoglycemic exposure in all groups of patients (Tables 3.4 3.5 and 3.6 ). This was associated with a lower (or at least not different) overall exposure to undesired glycemic levels (both hyper and hypoglycemia), as indicated by the AUCs values and the percentage of time spent in the normoglycemic range (the latter did not approach statistical significance only in children).

Total insulin dose was generally lower with the algorithm-based administration as compared to the standard therapy, and this was mainly attributable to a reduction in the basal insulin dose. In the adult population, total insulin dose resulted lower with the algorithm-based administration but the difference 
Table 3.4: Mean Different Indicator Values for the 10 Adults in the University of Virginia Simulator.

\begin{tabular}{|c|c|c|c|c|c|c|c|c|c|}
\hline \multicolumn{10}{|c|}{ ADULTS } \\
\hline & & AUC 2h & AUC 5h & AUC $7 \mathrm{~h}$ & $\begin{array}{l}\% \text { of time } \\
\text { Normo } 7 \mathrm{~h}\end{array}$ & $\begin{array}{l}\% \text { of time } \\
\text { Нypo } 5 \mathrm{~h}\end{array}$ & $\begin{array}{l}\% \text { of time } \\
\text { Нypo } 7 \mathrm{~h}\end{array}$ & Bolus & $\begin{array}{c}\text { Total IU } \\
7 \mathrm{~h}\end{array}$ \\
\hline \multirow{4}{*}{$40 \mathrm{~g}$} & Standard & 1 & 1 & 1 & 92.28 & 0.83 & 6.34 & 1 & 1 \\
\hline & Centroid & 0.57 & 0.53 & 0.15 & 97.91 & 0.00 & 0.00 & 0.88 & $0.88^{a}$ \\
\hline & Maximal-bolus & 0.08 & 0.08 & 0.02 & 99.55 & 0.00 & 0.00 & $1.54^{a b}$ & $0.92^{a}$ \\
\hline & $p$ value & 0.409 & 0.148 & 0.06 & 0.06 & 0.135 & 0.135 & $<0.001$ & $<0.001$ \\
\hline \multirow{4}{*}{$60 \mathrm{~g}$} & Standard & 1 & 1 & 1 & 81.59 & 2.83 & 9.90 & 1 & 1 \\
\hline & Centroid & 0.91 & 0.78 & 0.39 & $91.57^{a}$ & 0.00 & 0.71 & 1.01 & $0.89^{a}$ \\
\hline & Maximal-bolus & $0.62^{a}$ & $0.53^{a}$ & $0.28^{a}$ & $93.99^{a}$ & 0.00 & 0.93 & $1.38^{a b}$ & $0.93^{b}$ \\
\hline & $p$ value & 0.034 & 0.034 & 0.006 & 0.011 & 0.135 & 0.368 & $<0.001$ & 0.007 \\
\hline \multirow{4}{*}{$80 \mathrm{~g}$} & Standard & 1 & 1 & 1 & 71.30 & 3.53 & 11.80 & 1 & 1 \\
\hline & Centroid & 1.02 & 0.98 & 0.60 & $82.94^{a}$ & 0.00 & 0.59 & 0.97 & $0.88^{a}$ \\
\hline & Maximal-bolus & $0.73^{a b}$ & $0.66^{a b}$ & $0.41^{a}$ & $87.60^{a b}$ & 0.00 & 1.07 & $1.26^{a b}$ & $0.92^{b}$ \\
\hline & $p$ value & 0.002 & 0.002 & $<0.001$ & $<0.001$ & 0.135 & 0.204 & 0.001 & 0.007 \\
\hline \multirow{4}{*}{$100 \mathrm{~g}$} & Standard & 1 & 1 & 1 & 61.04 & 3.87 & 14.56 & 1 & 1 \\
\hline & Centroid & 0.96 & 0.91 & 0.63 & $76.41^{a}$ & 0.00 & $1.47^{a}$ & 0.99 & $0.87^{a}$ \\
\hline & Maximal-bolus & $0.80^{a b}$ & $0.73^{a b}$ & $0.50^{a}$ & $80.00^{a b}$ & 0.33 & $1.35^{a}$ & $1.17^{a b}$ & 0.90 \\
\hline & $p$ value & 0.007 & 0.007 & $<0.001$ & $<0.001$ & 0.368 & 0.043 & 0.001 & 0.002 \\
\hline \multirow{4}{*}{$120 \mathrm{~g}$} & Standard & 1 & 1 & 1 & 54.47 & 3.97 & 16.77 & 1 & 1 \\
\hline & Centroid & 1.05 & 1.03 & $0.78^{a}$ & $63.59^{a}$ & 0.00 & $4.75^{a}$ & 0.94 & $0.85^{a}$ \\
\hline & Maximal-bolus & 0.88 & 0.82 & $0.62^{a}$ & $69.24^{a}$ & 0.00 & $5.04^{a}$ & $1.10^{a b}$ & 0.88 \\
\hline & $p$ value & 0.184 & 0.111 & $<0.001$ & 0.002 & 0.135 & 0.005 & 0.039 & 0.002 \\
\hline \multirow{4}{*}{$140 \mathrm{~g}$} & Standard & 1 & 1 & 1 & 49.24 & 4.13 & 17.53 & 1 & 1 \\
\hline & Centroid & 1.09 & 1.09 & 0.86 & 54.89 & $0^{a}$ & $4.92^{a}$ & 0.92 & $0.83^{a}$ \\
\hline & Maximal-bolus & 0.96 & 0.91 & $0.73^{a}$ & 60.97 & $0^{a}$ & $6.08^{a}$ & 1.02 & 0.85 \\
\hline & $p$ value & 0.249 & 0.180 & 0.004 & 0.056 & 0.05 & $<0.001$ & 0.061 & 0.006 \\
\hline
\end{tabular}

${ }^{a} p<0.05$ versus standard bolus

${ }^{b} p<0.05$ versus centroid 
Open-loop Proposal: Combining basal-bolus insulin infusion for tight postpandial glucose control

Table 3.5: Mean Different Indicator Values for the 10 Adolescents in the University of Virginia Simulator

\begin{tabular}{|c|c|c|c|c|c|c|c|c|c|}
\hline \multicolumn{10}{|c|}{ ADOLESCENTS } \\
\hline & & AUC 2h & AUC 5h & AUC $7 \mathrm{~h}$ & $\begin{array}{l}\% \text { of time } \\
\text { Normo } 7 \mathrm{~h}\end{array}$ & $\begin{array}{l}\% \text { of time } \\
\text { Нуро } 5 \mathrm{~h}\end{array}$ & $\begin{array}{l}\% \text { of time } \\
\text { Нypo } 7 \mathrm{~h}\end{array}$ & Bolus & $\begin{array}{c}\text { Total IU } \\
7 \mathrm{~h}\end{array}$ \\
\hline \multirow{4}{*}{$40 \mathrm{~g}$} & Standard & 1 & 1 & 1 & 63.7 & 10.97 & 27.43 & 1 & 1 \\
\hline & Centroid & 1.38 & 0.91 & $0.43^{a}$ & $87.17^{a}$ & 0.00 & $1.42^{a}$ & 0.89 & 0.82 \\
\hline & Maximal-bolus & 1.25 & 0.83 & $0.47^{a}$ & $84.94^{a}$ & 2.10 & 5.84 & $1.18^{b}$ & 0.88 \\
\hline & $p$ value & 0.163 & 0.18 & 0.01 & 0.01 & 0.066 & 0.011 & 0.027 & 0.273 \\
\hline \multirow{4}{*}{$60 \mathrm{~g}$} & Standard & 1 & 1 & 1 & 56.22 & 11.63 & 28.38 & 1 & 1 \\
\hline & Centroid & 1.33 & 1.13 & $0.64^{a}$ & $74.06^{a}$ & $0^{a}$ & $5.11^{a}$ & 0.85 & $0.81^{a}$ \\
\hline & Maximal-bolus & 1.14 & 0.93 & $0.59^{a}$ & $68.41^{a}$ & $2.50^{a}$ & $13.92^{a}$ & 1.07 & 0.86 \\
\hline & $p$ value & 0.106 & 0.054 & $<0.001$ & 0.012 & 0.016 & 0.011 & 0.184 & 0.049 \\
\hline \multirow{4}{*}{$80 \mathrm{~g}$} & Standard & 1 & 1 & 1 & 48.15 & 12.33 & 29.81 & 1 & 1 \\
\hline & Centroid & 1.23 & 1.13 & $0.75^{a}$ & 58.50 & $0^{a}$ & $13.06^{a}$ & 0.85 & 0.80 \\
\hline & Maximal-bolus & 1.13 & 1.03 & $0.72^{a}$ & 58.08 & $2.13^{a}$ & $16.82^{a}$ & $0.99^{a b}$ & 0.84 \\
\hline & $p$ value & 0.138 & 0.205 & $<0.001$ & 0.236 & 0.01 & 0.023 & 0.045 & 0.061 \\
\hline \multirow{4}{*}{$100 \mathrm{~g}$} & Standard & 1 & 1 & 1 & 40.36 & 12.37 & 29.90 & 1 & 1 \\
\hline & Centroid & 1.22 & 1.22 & 0.89 & $49.74^{a}$ & $1.30^{a}$ & $16.27^{a}$ & $0.81^{a}$ & 0.77 \\
\hline & Maximal-bolus & 1.14 & 1.13 & $0.85^{a}$ & $49.22^{a}$ & $2.70^{a}$ & 19.24 & $0.92^{b}$ & 0.81 \\
\hline & $p$ value & 0.111 & 0.082 & 0.017 & 0.023 & 0.002 & 0.006 & 0.045 & 0.061 \\
\hline \multirow{4}{*}{$120 \mathrm{~g}$} & Standard & 1 & 1 & 1 & 36.13 & 12.03 & 29.19 & 1 & 1 \\
\hline & Centroid & 1.21 & 1.25 & 0.98 & $44.20^{a}$ & $1.40^{a}$ & $16.39^{a}$ & $0.78^{a}$ & 0.75 \\
\hline & Maximal-bolus & 1.15 & 1.19 & 0.94 & $48.79^{a}$ & $1.97^{a}$ & $15.11^{a}$ & $0.87^{b}$ & 0.78 \\
\hline & $p$ value & 0.096 & 0.061 & 0.067 & 0.002 & 0.004 & 0.023 & 0.045 & 0.273 \\
\hline
\end{tabular}

${ }^{a} p<0.05$ versus standard bolus

${ }^{b} p<0.05$ versus centroid 
Table 3.6: Mean Different Indicator Values for Nine Children Analyzed from Those Available in the University of Virginia Simulator

\begin{tabular}{|c|c|c|c|c|c|c|c|c|c|}
\hline \multicolumn{10}{|c|}{ CHILDREN } \\
\hline & & AUC 2h & AUC 5h & AUC $7 \mathrm{~h}$ & $\begin{array}{l}\% \text { of time } \\
\text { Normo } 7 \mathrm{~h}\end{array}$ & $\begin{array}{c}\% \text { of time } \\
\text { Hypo } 5 \mathrm{~h}\end{array}$ & $\begin{array}{c}\% \text { of time } \\
\text { Нypo } 7 \mathrm{~h}\end{array}$ & Bolus & $\begin{array}{c}\text { Total IU } \\
7 \mathrm{~h}\end{array}$ \\
\hline \multirow{4}{*}{$40 \mathrm{~g}$} & Standard & 1 & 1 & 1 & 66.77 & 10.26 & 22.75 & 1 & 1 \\
\hline & Centroid & 1.64 & 1.95 & 1.33 & 69.52 & $0.00^{a}$ & $0.00^{a}$ & 1.33 & $0.78^{a}$ \\
\hline & Maximal-bolus & 1.41 & 1.46 & 1.04 & 71.47 & $0.00^{a}$ & $0.00^{a}$ & 1.04 & $0.80^{a}$ \\
\hline & $p$ value & 0.278 & 0.129 & 0.642 & 0.923 & 0.041 & 0.003 & 0.06 & $<0.001$ \\
\hline \multirow{4}{*}{$60 \mathrm{~g}$} & Standard & 1 & 1 & 1 & 61.12 & 11.96 & 24.49 & 1 & 1 \\
\hline & Centroid & 1.36 & 1.27 & 0.87 & 73.24 & $0.00^{a}$ & $3.93^{a}$ & 0.87 & $0.78^{a}$ \\
\hline & Maximal-bolus & 1.29 & 1.27 & 0.90 & 72.34 & $0.00^{a}$ & $4.70^{a}$ & 0.90 & $0.80^{a}$ \\
\hline & $p$ value & 0.278 & 0.129 & 0.642 & 0.251 & 0.007 & 0.006 & 0.092 & $<0.001$ \\
\hline \multirow{4}{*}{$80 \mathrm{~g}$} & Standard & 1 & 1 & 1 & 51.31 & 14.04 & 30.48 & 1 & 1 \\
\hline & Centroid & $1.34^{a}$ & 1.36 & 1.00 & 61.52 & $0.04^{a}$ & $6.91^{a}$ & 1.00 & $0.77^{a}$ \\
\hline & Maximal-bolus & 1.27 & 1.27 & 0.95 & 64.71 & $0.30^{a}$ & $7.31^{a}$ & $0.95^{b}$ & $0.78^{a}$ \\
\hline & $p$ value & 0.031 & 0.154 & 0.187 & 0.164 & 0.002 & $<0.001$ & 0.019 & $<0.001$ \\
\hline \multirow{4}{*}{$100 \mathrm{~g}$} & Standard & 1 & 1 & 1 & 46.95 & 11.74 & 30.40 & 1 & 1 \\
\hline & Centroid & $1.33^{a c}$ & $1.41^{a c}$ & 1.08 & 54.50 & $0.67^{a}$ & $7.47^{a}$ & $1.08^{a}$ & $0.75^{a}$ \\
\hline & Maximal-bolus & 1.27 & 1.33 & 1.04 & 56.85 & $0.85^{a}$ & $7.86^{a}$ & $1.04^{b}$ & $0.77^{a}$ \\
\hline & $p$ value & $<0.001$ & $<0.001$ & 0.328 & 0.278 & 0.048 & 0.001 & 0.004 & $<0.001$ \\
\hline \multirow{4}{*}{$120 \mathrm{~g}$} & Standard & 1 & 1 & 1 & 40.83 & 10.74 & 30.19 & 1 & 1 \\
\hline & Centroid & $1.29^{a}$ & $1.41^{a}$ & 1.13 & 47.43 & $0.37^{a}$ & $7.44^{a}$ & $1.13^{a}$ & $0.74^{a}$ \\
\hline & Maximal-bolus & 1.26 & 1.37 & 1.11 & 48.19 & $0.44^{a}$ & $7.73^{a}$ & $1.11^{b}$ & $0.76^{a}$ \\
\hline & $p$ value & 0.006 & $<0.001$ & 0.209 & 0.406 & 0.031 & 0.001 & 0.019 & $<0.001$ \\
\hline
\end{tabular}


Open-loop Proposal: Combining basal-bolus insulin infusion for tight postpandial glucose control

was statistically significant only for the centroid solution (Table 3.4). Indeed, bolus increase with the maximal-bolus solution is compensated for reduction of basal insulin in the following hours. The increase in the bolus insulin dose was not seen in the adolescent and children populations in most of the tested meals. This fact may be explained by the high incidence of hypoglycemia in those groups as compared with the adult population.

Table 3.7 represents the glycemic variability during the postprandial 5 and 7 hours with the centroid, maximal-bolus and standard solution, showing significant improvement with temporal basal decrement solutions in all of the considered meals.

Table 3.7: Glycemic Variability over the Course of Five and Seven Hours

\begin{tabular}{|c|c|c|c|c|c|c|c|}
\hline & & \multicolumn{2}{|c|}{ ADULTS } & \multicolumn{2}{|c|}{ ADOLESCENTS } & \multicolumn{2}{|c|}{ CHILDREN } \\
\hline & & $\begin{array}{l}\text { Standard } \\
\text { deviation } \\
5 \mathrm{~h} \\
\end{array}$ & $\begin{array}{l}\text { Standard } \\
\text { deviation } \\
7 \mathrm{~h}\end{array}$ & $\begin{array}{l}\text { Standard } \\
\text { deviation } \\
5 \mathrm{~h}\end{array}$ & $\begin{array}{l}\text { Standard } \\
\text { deviation } \\
7 \mathrm{~h}\end{array}$ & $\begin{array}{l}\text { Standard } \\
\text { deviation } \\
5 \mathrm{~h}\end{array}$ & $\begin{array}{l}\text { Standard } \\
\text { deviation } \\
7 \mathrm{~h}\end{array}$ \\
\hline \multirow{4}{*}{$40 \mathrm{~g}$} & Standard & 13.48 & 15.91 & 24.9 & 28.2 & 27.19 & 27.99 \\
\hline & Centroid & $9.30^{a}$ & $9.72^{a}$ & $17.80^{a}$ & $20.13^{a}$ & $18.69^{a}$ & $19.53^{a}$ \\
\hline & Maximal-bolus & 11.59 & $11.12^{a}$ & $20.69^{a}$ & 21.71 & $19.17^{a}$ & $19.95^{a}$ \\
\hline & $p$ value & 0.009 & $<0.001$ & 0.002 & $<0.001$ & $<0.001$ & $<0.001$ \\
\hline \multirow{4}{*}{$60 \mathrm{~g}$} & Standard & 19.77 & 23.18 & 33.89 & 39.63 & 29.35 & 40.05 \\
\hline & Centroid & $14.68^{a}$ & $15.81^{a}$ & $27.53^{a}$ & $31.71^{a}$ & 28.74 & $30.22^{a}$ \\
\hline & Maximal-bolus & 16.92 & $16.93^{b}$ & $29.15^{a}$ & $32.03^{a}$ & 29.35 & $30.22^{a}$ \\
\hline & $p$ value & 0.002 & $<0.001$ & $<0.001$ & $<0.001$ & 0.154 & $<0.001$ \\
\hline \multirow{4}{*}{$80 \mathrm{~g}$} & Standard & 25.79 & 29.85 & 42 & 49.86 & 48.05 & 51.55 \\
\hline & Centroid & $20.22^{a}$ & $22.07^{a}$ & $36.47^{a}$ & $42.51^{a}$ & $39.63^{a}$ & $41.88^{a}$ \\
\hline & Maximal-bolus & $21.96^{a}$ & $22.37^{a}$ & $37.14^{a}$ & $42.13^{a}$ & $39.42^{a}$ & $40.96^{a}$ \\
\hline & $p$ value & 0.002 & $<0.001$ & 0.006 & $<0.001$ & $<0.001$ & $<0.001$ \\
\hline \multirow{4}{*}{$100 \mathrm{~g}$} & Standard & 31.67 & 35.96 & 50.23 & 59.78 & 57.05 & 61.99 \\
\hline & Centroid & $25.84^{a}$ & $27.82^{a}$ & $45.03^{a}$ & $52.99^{a}$ & $50.57^{a}$ & $54.45^{a}$ \\
\hline & Maximal-bolus & $27.00^{a}$ & $27.70^{a}$ & $45.16^{a}$ & $52.30^{a}$ & $50.11^{a}$ & $53.09^{a}$ \\
\hline & $p$ value & 0.001 & $<0.001$ & 0.006 & $<0.001$ & 0.01 & 0.005 \\
\hline \multirow{4}{*}{$120 \mathrm{~g}$} & Standard & 37.36 & 42.02 & 57.87 & 68.97 & 65.83 & 71.72 \\
\hline & Centroid & $31.00^{a}$ & $33.95^{a}$ & $53.35^{a}$ & $63.37^{a}$ & 60.27 & 65.2 \\
\hline & Maximal-bolus & $31.78^{a}$ & $33.32^{a}$ & $52.57^{a}$ & $61.91^{a}$ & 60.04 & 64.18 \\
\hline & $p$ value & $<0.001$ & $<0.001$ & $<0.001$ & $<0.001$ & 0.067 & 0.328 \\
\hline \multirow{4}{*}{$140 \mathrm{~g}$} & Standard & 42.37 & 47.66 & & & & \\
\hline & Centroid & $35.82^{a}$ & $39.33^{a}$ & & & & \\
\hline & Maximal-bolus & $36.30^{a}$ & $38.72^{a}$ & & & & \\
\hline & $p$ value & $<0.001$ & $<0.001$ & & & & \\
\hline
\end{tabular}


In Figures 3.10, 3.11 and 3.12 the mean glucose response of the adults, adolescents and children is shown for different carbohydrate content meals.
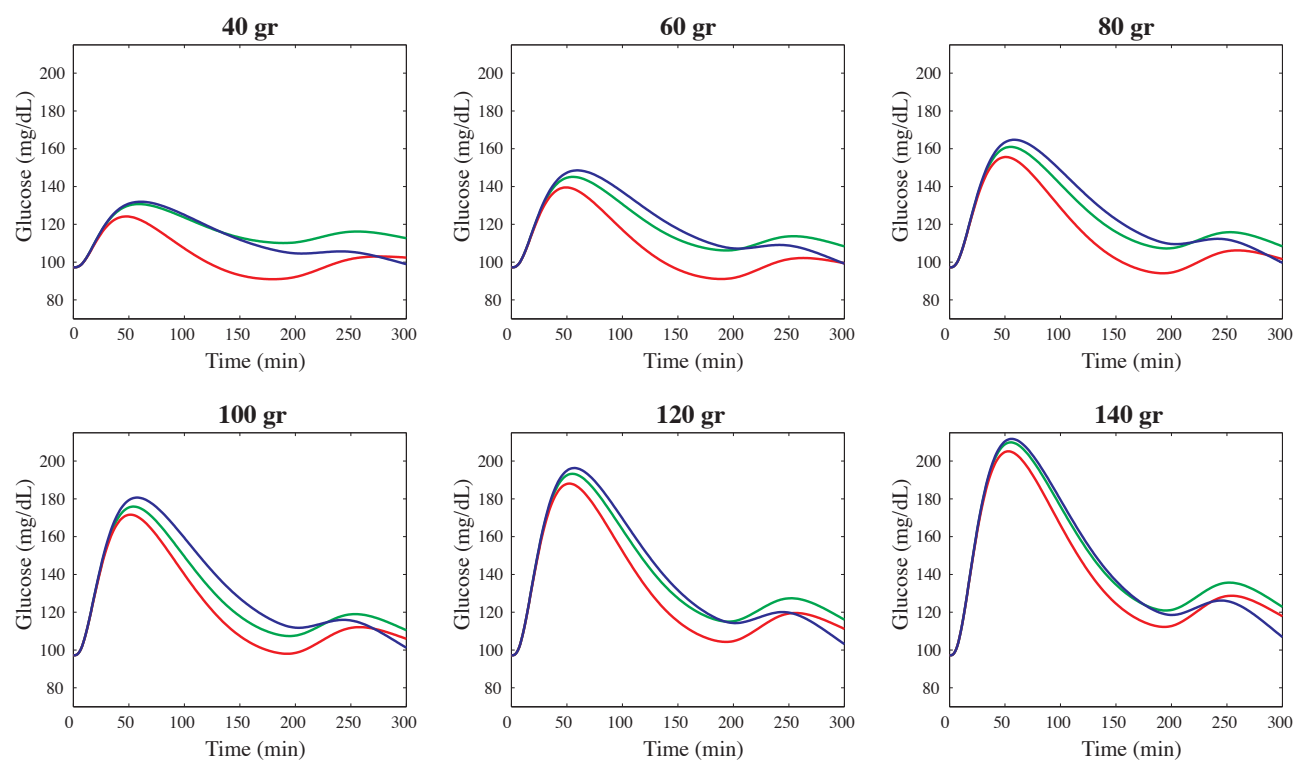

Figure 3.10: Mean glucose response of the 10 adults in the UVa simulator. The blue line represents the response applying the standard bolus, whereas the green and the red line correspond to the centroid and maximal-bolus solution, respectively. These latter solutions produce a flatter profile than the one observed with the standard bolus, avoiding late hypoglycemia. In addition, the peak in the glucose profile remains similar or even lower.

\subsubsection{Discussion of the in silico evaluation}

In this section, an in silico evaluation (needed step prior to clinical trials) of the algorithm proposed in Section 3.3 has been carried out. Results demonstrate the feasibility, efficacy and robustness of this approach. Algorithm-based bolus insulin administration is associated with lower hypoglycemic risk and less glycemic variability, as compared with standard strategy, in both the 5-h and 7-h postprandial horizons. Results indicate that, when looking for tight postprandial glucose control, a parallel reduction in basal insulin dose is 
Open-loop Proposal: Combining basal-bolus insulin infusion for tight postpandial glucose control
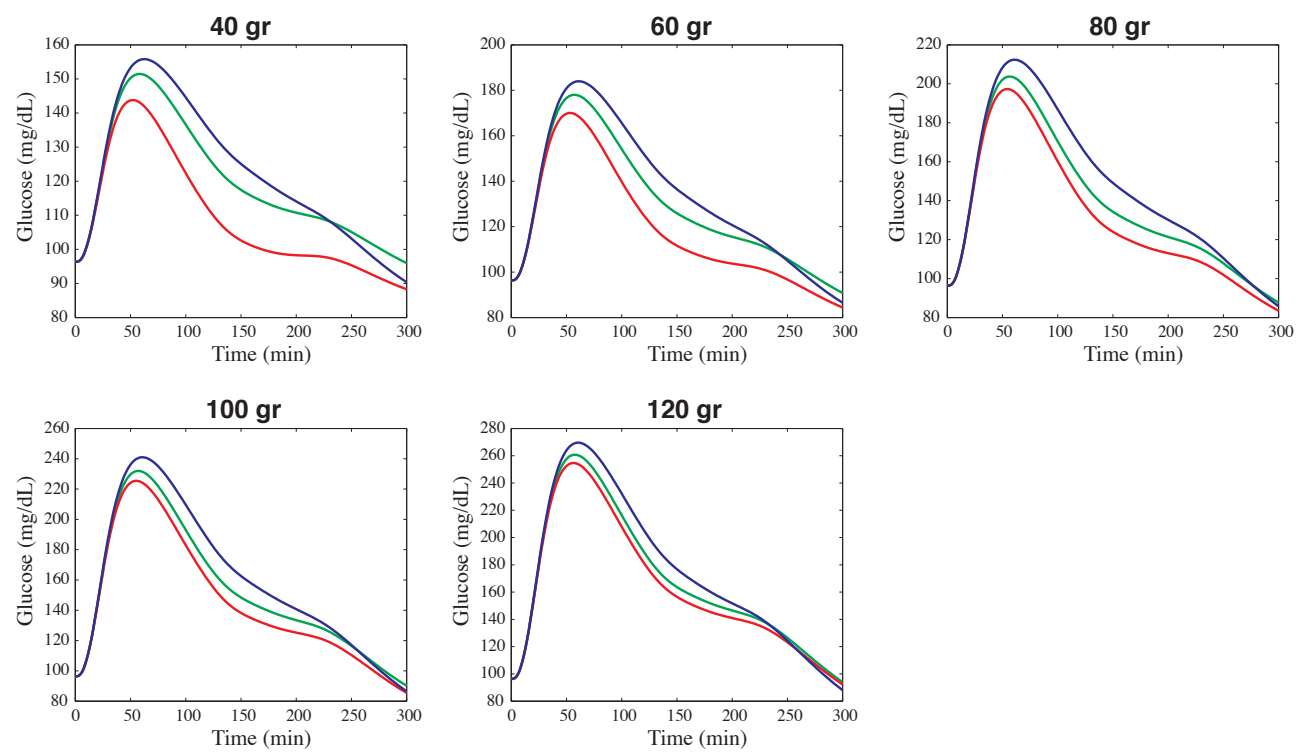

Figure 3.11: Mean glucose response of the 10 adolescents in the UVa simulator. The blue line represents the response applying the standard bolus, whereas the green and the red line correspond to the centroid and maximal-bolus solution, respectively. These latter solutions produce a flatter glucose profile than the one observed with the standard bolus, avoiding late hypoglycemia. The peak in the glucose profile using any of the solutions is similar. 

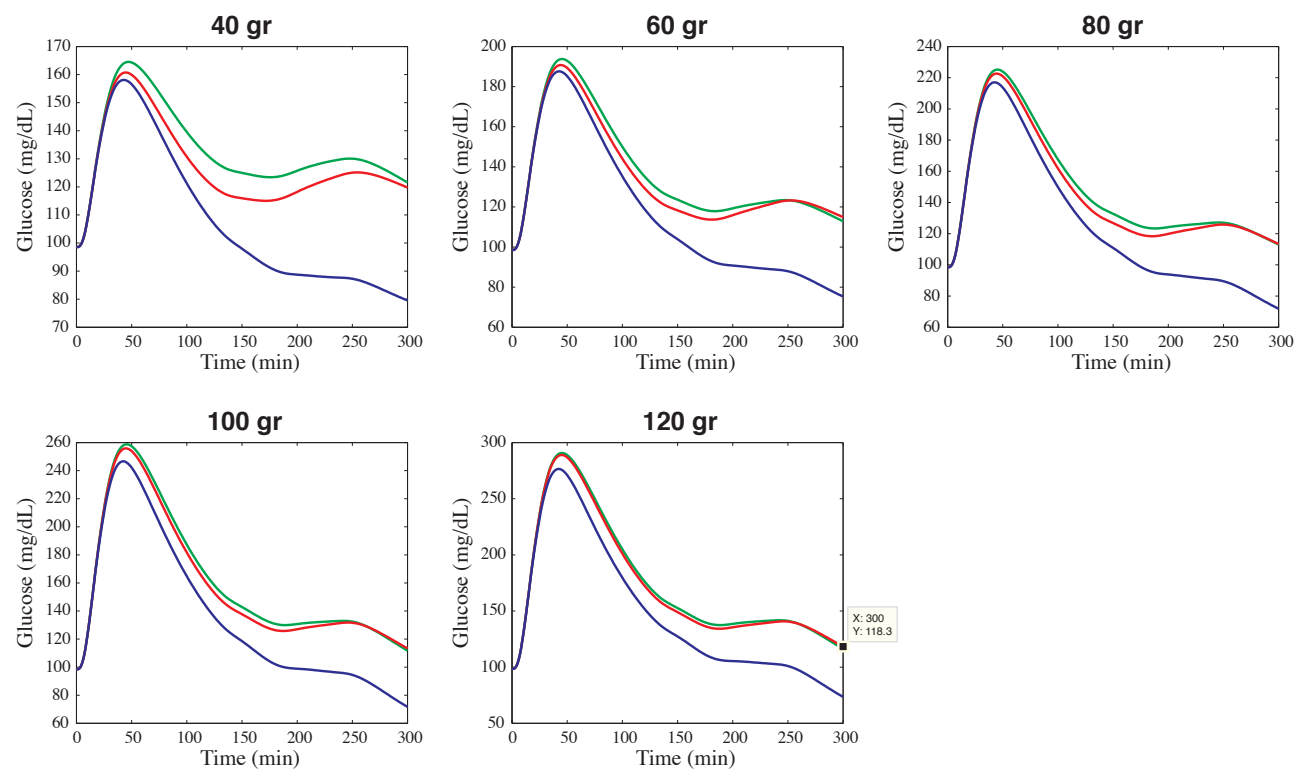

Figure 3.12: Mean glucose response of nine children analyzed from those available in the UVa simulator. The blue line represents the response applying the standard bolus, whereas the green and the red line correspond to the centroid and maximal-bolus solution, respectively. These latter solutions, although producing a slightly higher peak in the glucose profile than the standard bolus, achieve a flatter glucose profile, avoiding the severe late hypoglycemia. 
Open-loop Proposal: Combining basal-bolus insulin infusion for tight postpandial glucose control

required to limit late post-absorptive hypoglycemia, especially for meals with higher carbohydrates content.

Strength of the proposed method is its robustness. The use of a model significantly different from the virtual patient for the identification of the patient's postprandial behavior shows the feasibility of the method in spite of imperfect glucose predictions, due for instance to intra-patient variability. Robustness of the solution could be further increased, if needed, with explicit consideration of intra-patient variability (as interval quantities in model parameters) or considering uncertainty in the carbohydrates content of the meal in the computation of the feasible solution set with SIVIA (that would yield smaller feasible sets and thus more constrained solutions). This is a unique feature of the presented algorithm. Another one is the possibility to determine, in a non-heuristic way, the feasible insulin administration modes for a given meal, which could be included in smarter insulin pumps in the future.

The main limitation of this study is that its results may not apply to the real diabetic population in daily life. Adult virtual patients are built on data from real patients response to a specific (and non physiologic) mixed meal [13, not necessarily representative of the postprandial response to a meal of different composition. Extrapolation of results from virtual adolescents and children to the real population should be done even more cautiously. Indeed, to the best of our knowledge, no published data are available on traced mixed meal postprandial response of non-adult diabetic people. Data published so far for young people have been obtained from oral glucose tolerance test (OGTT) $)^{1}$ [161, but results are certainly not equivalent to a mixed meal.

Finally, identification of the patient model will definitely require specific protocols to be followed by the patient during several days to avoid nonidentifiability issues. This can be an inconvenient for the patient.

In conclusion, despite its limitations, the results of this in silico validation were promising enough to carry out a clinical validation of the algorithm to

\footnotetext{
${ }^{1} \mathrm{~A}$ medical test where a standard dose of glucose is ingested by mouth and blood samples are taken afterwards to determine how quickly glucose is cleared from the blood.
} 
test its feasibility to be implemented in current insulin pumps. The protocol and results of that clinical trial are provided in next section.

\subsection{Clinical validation}

Results of the in silico validation of the set inversion-based algorithm for postprandrial control, encouraged its evaluation in clinical practice by the team. In that study, the proposed algorithm was compared with standard strategy for counteracting meals based on the I:C ratio.

The study was a randomized, prospective, single-center, double-blinded, two-way crossover study. It involved 12 people ( 9 women and 3 men) with T1DM under long-term intensive insulin treatment with CSII. The study was done in the Clinic University Hospital of Valencia, Spain, under controlled circumstances after an ambulatory period registering data using a CGMS. It was carried out according to the Declaration of Helsinki after written informed consent was obtained from all subjects and approved by the local institutional review board.

This study is registered at ClinicalTrials.gov with trial registration number NCT01550809. The research leading to these results received funding from the European Union Seventh Framework Programme (FP7/2007/2013) under grant agreement 252085 and from the Spanish Ministry of Science under grants DPI2010-20764-C02-01 and DPI2011-28112-C04-01. The team was composed by Dr. Paolo Rossetti, Dr. Jorge Bondia, Dr. Javier AmpudiaBlasco, Dr. Juan F. Ascaso, Dr. Josep Vehi, Dr. Alejandro Laguna and Ana Revert. P.R. contributed to the study concept and design, wrote the study protocol, performed meal tests, researched and analyzed the data, F.J.A-B. contributed to the study concept and design and reviewed the study protocol, A.L. and A.R. researched and analyzed the data, J.F.A. supervised the protocol development and the research, J.V. contributed to the study concept and design and reviewed the study protocol, J.B. contributed to the study concept and design, supervised the protocol development and the research, analyzed the data. 
Open-loop Proposal: Combining basal-bolus insulin infusion for tight postpandial glucose control

More detail about the clinical protocol and results of the study can be found in [140.

\subsubsection{Characteristics of the study}

The anthropometric, demographic and metabolic parameters of the subjects that participated in the study are showed in Table 3.8.

The study consisted of four 5-hour mixed-meal tests, two of $40 \mathrm{~g}$ carbohydrates and two of $100 \mathrm{~g}$ carbohydrates. For each type of meal the standard therapy based on the individual I:C ratio was compared to the therapy suggested by SIVIA algorithm, in consecutive weeks. Both the physicians and patients were blinded regarding the bolus administration procedure during test meals. Prior to each block of meal tests, subjects underwent at least two outpatient 6-day periods of CGMS monitoring for the identification (the first 3 days) and validation (the last 3 days) of an individualized model to be used in the prediction of the 5 -h postprandial period using a clinical protocol derived by the team from a qualitative analysis of optimal experiment design results for a variety of models [95] (similarly to Section 3.4.1 for Hovorka model).

$20 \%$ uncertainty in insulin sensitivity and $10 \%$ uncertainty in the grams of carbohydrates was considered in the interval model used in SIVIA algorithm. In that way hard constraints were applied to the punctual model whereas soft constraints were used for the interval model [21]. The selection of the specific basal-bolus combination from the $3 \mathrm{D}$ set of solutions provided by the SIVIA algorithm was done using the maximum-bolus solution. Remark that the solution set was considered here as a whole and no prior bolus mode was selected. In that way, the solution set may contain profiles either increasing (dual-wave bolus) or decreasing (temporal basal decrement) postprandial basal rate as required to fulfill the constraints for the specific patient.

For each study, subjects were admitted to the Clinic University Hospital of Valencia at 09:00 $\mathrm{h}$ and all mixed-meal tests started at 13:00 $\mathrm{h}$ with the ingestion of a meal. Comparable initial metabolic conditions among the tests were guaranteed by a prior intravenous infusion of regular human insulin 
following a feedback procedure to maintain plasma glucose (PG) close to 90 $m g / d l$ before the starting of the study. In order to avoid hypoglycemia during test (5 hours), a controlled glucose infusion was started if PG fell below 75 $m g / d l$, and the premeal glycemic levels were maintained.

Table 3.8: Demographic, anthropometric and metabolic parameters of the patients participating in the study

\begin{tabular}{lccccc}
\hline & Age & BMI $\left(\mathrm{kg} / \mathrm{m}^{2}\right)$ & HBA1c $(\%)$ & Nominal basal $(\mathrm{IU} / \mathrm{h})$ & $\mathrm{I}: \mathrm{C}(\mathrm{IU} / 10 \mathrm{~g})$ \\
\hline Mean & 41.8 & 25.1 & 8.0 & 0.8 & 1.3 \\
SD & 7.3 & 2.8 & 0.6 & 0.3 & 0.5 \\
\hline
\end{tabular}

\subsubsection{Metrics used and results of the clinical validation}

The metrics used for the clinical validation were similar than the ones used in silico. In that way, the $\mathrm{AUC}$ of $\mathrm{PG}$ during the whole experiment $(A U C-$ $\left.P G_{0-5 h}\right)$ was calculated for each of the four tests. It is a measure of the overall glucose-lowering efficacy of the postprandial strategy. Moreover, the AUC of the glucose infusion rate $\left(A U C-G I R_{0-5 h}\right)$ which represents a measure of hypoglycemic exposure was also calculated. The most effective postprandial strategy is determined then by the lower value of $A U C-P G_{0-5 h}$ without increasing $A U C-G I R_{0-5 h}$. Additionally, the AUC of PG above the threshold of $140 \mathrm{mg} / \mathrm{dl}(A U C-P G>140)$ was computed as an indicator of meal-induced hyperglycemic risk.

Variability of the postprandial glycemic response was calculated as the coefficient of variation (CV) of the $A U C-C G M S_{0-5 h}$ and of the $A U C-$ $P G_{0-5 h}$. Glucose concentrations at t0 $(P G-t 0)$, the mean prandial insulin dose (Insulin dose $\left.\mathrm{d}_{0-5 h}\right)$, and their respective CVs $\left(C V-G_{t 0}, C V-P G_{t 0}\right.$, and CV-Insulin dose $_{0-5 h}$ ) were computed as well, as potentially related to variability of postprandial glucose.

All data were subjected to repeated-measures analysis of variance with Huynh-Feldt adjustment for nonsphericity [180]. The analysis of variance 
Open-loop Proposal: Combining basal-bolus insulin infusion for tight postpandial glucose control

model included the sequence of studies as a between-subjects factor, whereas test condition (maximal-bolus vs. standard), carbohydrates content of the meal (40 g and $100 \mathrm{~g}$ carbohydrates conditions), and time (where appropriate) were the within-subjects factors. Subjects were entered in the model as random factors nested into the sequence. Post hoc comparisons with a nominal significance level of 0.05 (Newman-Keuls test) were carried out to pinpoint specific differences on significant interaction terms. The coefficient of determination $R^{2}$ was reported as a measure of the proportion of variability in the glycemic response that was accounted for by the statistical model.

As it can be inferred from Table 3.9, the postprandial glycemic control was similar independently of the doses strategy followed (standard therapy and the maximal-bolus solution from SIVIA). In that way the overall 5 hours glycemic exposure $\left(A U C-P G_{0-5 h}\right)$, the hypoglycemic risk $\left(A U C-G I R_{0-5 h}\right)$, and the hyperglycemic risk (AUC-PG $>140)$ were not different for the different strategies. This fact contrasts with the results in insulin dose. The maximal-bolus strategy resulted in a clinically although not statistically significant approximately $30 \%$ greater mean insulin dose (bolus +5 -hours basal) compared with the standard therapy. The bolus dose accounted for the whole increment in the $40 \mathrm{~g}$ carbohydrates meals whereas in the $100 \mathrm{~g}$ carbohydrates meals both the basal insulin and the bolus insulin were equally increased.

With respect to variability, despite the feedback procedure that guaranteed equal prepandial PG values, the postprandial glucose response varied in a wide range with both the maximal-bolus (median CV, 14.7\%; interquartile range, $4.5-27.9 \%$ ) and the standard (median $\mathrm{CV}, 5.4 \%$; interquartile range, $3.3-12.7 \%$ ) (Table 3.10). Glucose variability was significantly greater with the maximal-bolus, likely because of the wider range of insulin doses administered (Table 3.9).

\subsubsection{Discussion of the clinical validation}

The purpose of this proof-of-concept study was to validate the non-heuristic interval analysis based algorithm for determining the best basal-bolus com- 
Table 3.9: Insulin Dose and the Area Under the Curves of Plasma Glucose, Plasma Glucose Above $140 \mathrm{mg} / \mathrm{dl}$, and Glucose Infusion Rate of the 5-h Postprandial Period for the maximal-bolus solution and the standard therapy

\begin{tabular}{|c|c|c|c|c|c|}
\hline & \multicolumn{2}{|c|}{$40 \mathrm{~g}$ of carbohydrates } & \multicolumn{2}{|c|}{$100 \mathrm{~g}$ of carbohydrates } & \multirow[b]{2}{*}{$p$ value } \\
\hline & Maximal-bolus & Standard & Maximal-bolus & Standard & \\
\hline 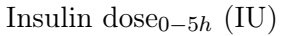 & $9.9 \pm 3.4$ & $7.4 \pm 2.1$ & $18.0 \pm 6.7$ & $13.8 \pm 4.3$ & 0.32 \\
\hline Bolus dose (IU) & $(7.1 \pm 2.8)$ & $(4.3 \pm 1.5)$ & $(13.9 \pm 7.1)$ & $(10.8 \pm 3.8)$ & \\
\hline AUC-PG ${ }_{0-5 h}$ & $585.5 \pm 127.5$ & $689.2 \pm 180.7$ & $752.1 \pm 237.7$ & $760.0 \pm 263.2$ & 0.07 \\
\hline AUC-GIR $_{0-5 h}$ & $150.1 \pm 134.5$ & $54.0 \pm 87.4$ & $88.9 \pm 195.5$ & $56.0 \pm 121.1$ & 0.45 \\
\hline AUC-PG $>140 \mathrm{mg} / \mathrm{dl}$ & $37.2 \pm 63.5$ & $77.5 \pm 123.8$ & $141.3 \pm 182.8$ & $144.6 \pm 217.7$ & 0.29 \\
\hline
\end{tabular}

Table 3.10: Intrasubject Variability of the Postprandial Glycemic Response Reported as the Intra-Individual Coefficient of Variation of the 5-h Postprandial Period

\begin{tabular}{lccc}
\hline \multicolumn{4}{c}{$C V$} \\
\hline & $\mathrm{Gt0} / \mathrm{PGt0}(\mathrm{mg} / \mathrm{dl})$ & Insulin dose $(\mathrm{IU})$ & $\mathrm{AUC}_{0-5 h}(\mathrm{mg} / \mathrm{dl} \cdot \mathrm{h})$ \\
Overall & $6.4 \pm 2.5 \%(101.9 \pm 4.1)$ & $40.1 \pm 11.6 \%(12.3 \pm 3.6)$ & $14.5 \pm 10.5 \%(696.7 \pm 175.3)$ \\
Maximal-bolus & $6.7 \pm 3.7 \%(101.2 \pm 4.4)$ & $39.5 \pm 24.9 \%(13.9 \pm 4.4)$ & $16.7 \pm 15.3 \%(668.8 \pm 162.7)$ \\
Standard & $5.6 \pm 5.0 \%(102.5 \pm 5.5)$ & $42.8 \pm 3.3 \%(10.6 \pm 3.2)$ & $10.1 \pm 12.5 \%(724.6 \pm 198.7)$ \\
$p$ value & $0.36(0.43)$ & $0.65(0.007)$ & $0.04(0.07)$ \\
\hline
\end{tabular}

bination leading to a good postprandial control, already tested in simulations using the FDA-accepted UVa simulator, in CSII-treated patients with T1DM. Results show that the proposed strategy for insulin delivery provides a postprandial glycemic control similar to that achieved by standard bolus calculators. Taking into account that currently postprandial control is still a heuristic process depending on the expertise of the patient and the physician, these results are encouraging and open the way to larger clinical studies aimed at validating less user-dependent strategies for prandial insulin dosing. The study validates also the feasibility of using CGMS measures for the automatic insulin dosing.

Several are the causes for the differences found between the in silico study results and those from the clinical trial, and all are related to the identification process. Remark that SIVIA algorithm guarantees the fulfillment of the constraints as far as the prediction model is reliable. Although to test the robustness of the algorithm a model different from the one used in UVa 
Open-loop Proposal: Combining basal-bolus insulin infusion for tight postpandial glucose control

simulator was used for obtaining the identified prediction models (Section 3.4.1), the postprandial response of each virtual patient in the simulator only depends on the carbohydrates quantity. However, not only the quantity of the carbohydrates but also the proteins and fats content of the meal can significantly affect glycemic postprandial response. Available models in literature are presumably specific to the meal used in the experiment and may not apply to meals of different composition, limiting prediction capabilities of the individual model.

Moreover, another factor not considered in the in silico validation was intrapatient variability with resulted to be huge in the clinical test. In that way, in spite of keeping into control the main known sources of variability (meal composition, prepandial glycemia and accuracy of the glucose measurments), clinically significant different insulin doses using different strategies (30\% greater with the maximal-bolus strategy compared with the standard therapy (see Tables 3.9 and 3.10 achieved similar glycemic control. This fact was also observed at the individual level in some cases. This must be regarded as "unexplained" variability, which makes it very difficult to control postprandial glucose response in open loop for some patients, whatever the model used for insulin dosing: the bolus advisors currently implemented in clinical practice, or new proposals. In that respect, one of the advantages of the proposed strategy is that uncertainty can be considered in the SIVIA algorithm. In this way, if the causes of variability could be characterized, they would be easily incorporated in the algorithm as interval parameters aiming at more robust insulin dosing according to the individual patient model. Further work is thus necessary in modeling postprandial behavior and the characterization of variability.

\subsection{Conclusions}

In this chapter, an innovative strategy for calculating the most appropriate combination of basal and bolus insulin for a good postmeal glucose control has been thoroughly presented. The set inversion methods based on interval analysis are applied to determine, for a given meal, which bolus-administration 
mode will yield a glucose response fulfilling the IDF guidelines of postprandial control.

The algorithm was first evaluated in silico with the adults, adolescents and children in the UVa simulator demonstrating robustness with respect to the patient model. The promising results lead to the development of a clinical protocol for the validation of the algorithm in vivo. The clinical trial demonstrated the feasibility of the algorithm using CGMS measurements for postprandial insulin delivery, showing similar results than those using traditional therapies. The fact that a non-heuristic CGMS-based insulin delivery algorithm is capable of matching the performance of currently heuristic strategies is encouraging. However, some limitations were found that explain the differences with respect the in silico test.

The main problem is high intra-subject variability, that is a barrier for obtaining good prediction models to be used by the algorithm. Most of the causes of that high variability remains unexplained and more research should be done to determine its nature. This limitation is difficulties to overcome in open-loop strategies and it encourages the use of closed-loop strategies as presented in the next chapter.

Despite its limitations, the proposed algorithm has some features that provide interesting advantages. The first one is that it can consider uncertainty in the models' parameters. This means that individual variations in insulin sensitivity, meal absorption, or errors in the estimation of the amount of ingested carbohydrates (among other factors) can be considered in insulin dosing. Moreover, it provides not a single but a set of solutions that, according to the model, are designed to maintain the patient in a prespecified glycemic range. These features may be relevant in clinical practice because, if the individual models were good, they would allow for a more robust and safer insulin administration.

In next chapter, the second proposal of this thesis is presented. It consists of an algorithm called SAFE which purpose is again to improve postprandial control using CGMS measurements, gaining in robustness and avoiding late 
Open-loop Proposal: Combining basal-bolus insulin infusion for tight postpandial glucose control

hypoglycemia events. The algorithm is thought to be added to closed-loop glucose control schemes. 



\section{Closed-loop strategy: A ref- erence conditioning method for the reduction of hypogly- cemia}

In last chapter an open-loop algorithm to improve postprandial glucose control under uncertainty was presented. It's performance was tested both in silico and in vivo. The result demonstrated the feasibility of using an automatic non-heuristic CGMS-based algorithm for insulin delivery. However the results obtained in the clinical validation did not significantly improve those obtained with the standard therapy. The main barrier was high intrasubject variability which accounts for the greatest part of unpredictability of postprandial glycemic control and remains a barrier for the implementation of more effective open-loop insulin treatments.

In an attempt to overcome this problem and due to its potential advantages with respect to open-loop proposals a closed-loop strategy is presented in this chapter. The main objective of this proposal is to help in avoiding one of the main challenges of artificial pancreas: over-reaction leading to hypoglycemia, especially in the late post prandial period. An original proposal based on sliding mode reference conditioning ideas is presented as a way to reduce hypoglycemia events induced by a closed-loop glucose controller. The method is inspired in the intuitive advantages of two-step constrained control 
algorithms. It acts on the glucose reference sent to the main controller, shaping it so as to avoid violating given constraints on the insulin-on-board. Some distinctive features of the proposed strategy are that (i) it provides a safety layer which can be adjusted according to medical criteria, (ii) it can be added to closed-loop controllers of any nature, (iii) it is robust against sensor failures and over-estimated prandial insulin doses, (iv) it can handle non-linear models.

This chapter is organized as follows. Section 4.2 revises the theoretical concepts behind this proposal. Section 4.3 presents the method design and analysis, showing its robustness properties and presenting some discussion about the definition of IOB bounds. In Section 4.4 some simulations are carried out to illustrate the robustness of the strategy against sensor failures and priming bolus over-estimations. Moreover, the proposal is evaluated in silico using the 10 adult patients available in the educational version of the the Food and Drug Administration-accepted University of Virginia simulator (UVA Simulator) [88. Finally, in Section 4.5 a set of conclusions is provided.

Part of the contents of this chapter has been published in:

- A. Revert, F. Garelli, J. Pico, H. De Battista, P. Rossetti, J. Vehi, J. Bondia. Safety Auxiliary Feedback Element for the Artificial Pancreas in Type 1 Diabetes, IEEE Transactions on Biomedical Engineering , 60(8), 2113- 2122, 2013 [137]

\subsection{Introduction}

As it has been discussed in Chapter 2, in the last 10-15 years the development of CGMS has fostered research in the artificial pancreas and several experiments of CGMS-based automated insulin infusion have been performed. Although different controllers have been proposed, those with more clinical evidence are PID [153, 103] controllers and algorithms such as MPC [69]. In an attempt to improve the performance of the controllers, and the control of postprandial glycemia excursions avoiding overcorrection and subsequent hypoglycemia, several schemes are being used. In this way, feedforward 
Closed loop strategy: A reference conditioning method for the reduction of hypoglycemia

strategies with meal announcement and different safety strategies such as modular control-to-range, the addition of safety constraints in the residual insulin activity, the so-called insulin-on-board (IOB), or the addition of an insulin feedback are being incorporated to the control schemes (see Section 2.4 .2 .

However, the problem of overcorrection has not been solved yet in a satisfactory and universal way. Only solutions that apply to specific scenarios have been successfully tested [52, 122]. Therefore, further investigation needs to be perform in this field.

Specifically, it seems important to propose and evaluate alternative methods to implement safety constraints on IOB which can be applied to any glucose closed-loop system. Indeed, two general approaches can be found in control systems literature in order to deal with constraints [108, 121]:

- one-step approaches, in which knowledge of constraints is explicitly exploited, and constraints accounted for, during control law design. These are mostly MPC strategies.

- two-step algorithms, originally inspired in reference governors schemes for industrial controllers.

The latter approach can be illustrated by the premise: "design the controller ignoring limitations, and then add a compensation to minimize adverse effects of limitations on closed-loop performance" [86]. This leads to some separation in the controller such that one part is devoted to achieving nominal performance and the other part is devoted to constraint handling. This feature allows the development of universal solutions that are applicable regardless of the control scheme chosen. Thus, all the know-how on classical control and tuning techniques can be used for the main control loop design.

In the case of glucose control, a two-step approach would permit exploiting clinical evidence and experience on other valuable control strategies which are not able to deal with IOB constraints by their own, such as ePID and ePIDIFB [114, 27], or even non-linear control laws which take advantage of advanced 
physiological and pharmacokinetic dynamics knowledge. Despite the intuitive advantages attributed to two-step algorithms [156], they are usually criticized for being ad hoc methods without the background of a well-established theory.

In this chapter, sliding mode reference conditioning (SMRC) previously successfully used in different fields [56, 58, 128, 165, 57] is applied to the design of a safety algorithm to reduce hypoglycemia events in closed-loop glucose control. It follows the two-step approximation to constrained control with the advantage of providing a rigorous methodology design and robustness against sensor failures. Indeed, differing from most two-step algorithms originally conceived as anti-windup methods for linear and biproper controllers, the proposed method can deal with both biproper/strictly-proper controllers and linear/nonlinear controllers or IOB estimators. Furthermore it does not require the model of the controller and IOB estimator but only their relative degree (generally constant and known a priori), and provides insensitive IOB limits to matched disturbances and sensor errors.

Some initial approaches based on conventional sliding mode control have been reported both with [3] and without [82] meal announcement. In contrast, in this proposal sliding modes are not established within the main control loop but in an auxiliary software-based loop, which can operate at much faster sampling rates than the ones allowed by current glucose monitors. In this way, given a closed-loop controller (which can be of any nature), an outer control loop is added so as to impose constraints on the IOB. This loop, based on SMRC, is only active when the IOB amounts to undesirable values. It acts on the reference of the main controller, shaping it so as to avoid the violation of the constraints. Both upper and lower constraints in IOB can be defined, reducing the hypoglycemia risk but also avoiding too high blood glucose values, mainly in the late-postprandial period, acting as a second layer control. The IOB is estimated through a subcutaneous insulin absorption model and different IOB limits can be imposed depending on the ingested meal and on the estimation of the insulin sensitivity for each patient. The main advantage of this scheme is that it does not affect the design of the inner controller, which could be designed previously and in an independent way. 
Closed loop strategy: A reference conditioning method for the reduction of hypoglycemia

\subsection{Theoretical framework}

In order to address the glucose control problem with constrained IOB from a general framework, in this section some important concepts on invariance control and sliding regimes are reviewed. In particular, the necessary conditions to confine a non-linear dynamical system to an invariant region of the state-space are presented, and compared with the equivalent continuous dynamics of a system operating in sliding mode (SM), i.e., when the system input consists of a high frequency discontinuous signal.

\subsubsection{Invariance control}

Let the system

$$
\left\{\begin{array}{c}
\frac{d x(t)}{d t}=f(x, d)+g(x) w(t) \\
y(t)=h_{1}(x) \\
v(t)=h_{2}(x)
\end{array}\right.
$$

where $x(t) \in \mathbb{R}^{n}$ is the state vector, $w(t) \in \mathbb{R}$ is a control input and $d(t) \in \mathbb{R}^{n}$ an unmeasured perturbation which can represent either parametric uncertainties or external non-structured disturbances. $f(x, d): \mathbb{R}^{n} \times \mathbb{R}^{n} \longrightarrow \mathbb{R}^{n}$ and $g(x): \mathbb{R}^{n} \longrightarrow \mathbb{R}^{n}$ are vector fields and $h_{1}(x), h_{2}(x): \mathbb{R}^{n} \longrightarrow \mathbb{R}$ scalar fields.

Variables $y(t)$ and $v(t)$ are both real-valued system outputs, $y(t)$ being the main controlled variable and $v(t)$ a variable to be bounded so as to belong to the set:

$$
\Sigma=\left\{x(t) \mid \sigma(t)=v(t)-v^{*}(t) \leq 0\right\},
$$

with $v^{*}(t)$ the bound imposed to $v(t)$.

Thus, the goal is to find a control action $w(t)$ such that the region $\Sigma$ becomes invariant (i.e., trajectories originating in $\Sigma$ remain in $\Sigma$ for all times $t$ ), while $y(t)$ is driven as close as possible to its desired value $r$. To ensure 
the invariance of $\Sigma$, the control action $w(t)$ must guarantee that the righthand-side of the first equation in system (4.1) points to the interior of $\Sigma$ at all points on the border surface $\partial \Sigma=\{x(t) \mid \sigma(t)=0\}$. Figure 4.1 is a graphical representation of this invariance condition. Mathematically, this is verified if the implicit invariance condition [7, 104]:

$$
\inf _{w} \dot{\sigma}(t) \leqslant 0, \text { with } x(t) \in \partial \Sigma
$$

holds.

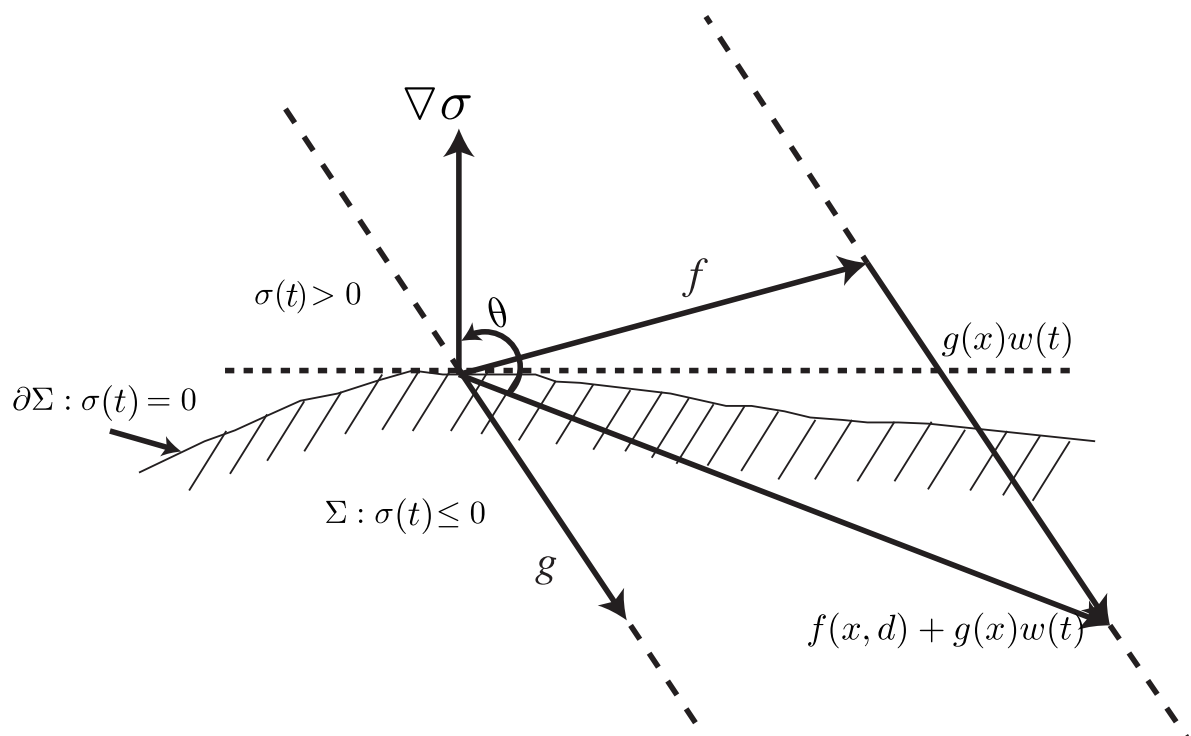

Figure 4.1: Invariance condition. Adapted from [128].

From equations (4.1), using Lie-derivatives, we have

$$
\begin{aligned}
w(t) & =\left(L_{g} \sigma(t)\right)^{-1}\left[\dot{\sigma}(t)-L_{f} \sigma(t)+\dot{v}^{*}(t)\right] \\
& =w^{\sigma}+\left(L_{g} \sigma(t)\right)^{-1} \dot{\sigma}(t)
\end{aligned}
$$

with $w^{\sigma}=\left(L_{g} \sigma(t)\right)^{-1}\left[-L_{f} \sigma(t)+\dot{v}^{*}(t)\right]$. Since $w(t)$ must be chosen so as to fit equation 4.3 , it must verify $\left(w(t)-w^{\sigma}\right) L_{g} \sigma(t) \leqslant 0, \forall x(t) \in \partial \Sigma$.

Hence, the explicit invariance control for system 4.1) is obtained 
Closed loop strategy: A reference conditioning method for the reduction of hypoglycemia

$$
\begin{aligned}
& w(t) \in w \in \mathbb{R} \mid w<w^{\sigma} \text { if }\left(x(t) \in \partial \Sigma \wedge L_{g} \sigma(t)>0\right) \\
& w(t) \in w \in \mathbb{R} \mid w>w^{\sigma} \text { if }\left(x(t) \in \partial \Sigma \wedge L_{g} \sigma(t)<0\right) \\
& w(t)=0 \text { if }(x(t) \in \Sigma \backslash \partial \Sigma)
\end{aligned}
$$

with $L_{f} \sigma(t)$ assumed to be positive without loss of generality.

Note that condition $L_{g} \sigma(t)=\frac{\delta \sigma(t)}{\delta x(t)} g(x) \neq 0$ must hold on $\partial \Sigma$ for $w^{\sigma}$ to exist and invariance control be feasible. Observe also that once the surface $\partial \Sigma$ and the control field $g(x)$ are defined, only one of the two inequalities holds, i.e., $L_{g} \sigma(t)$ remains either positive or negative but never changes its sign.

\subsubsection{Finite-time invariance achievement via Sliding Mode Reference Conditioning}

The concept of reference conditioning to achieve a realizable reference arises in the context of constrained control systems. Specifically, Hanus and Walgama [64, 169] applied this kind of solutions to solve the problem of actuator saturation (windup) in linear controllers. Based on these approaches and getting advance of the possibilities of sliding modes, Mantz and colleagues [102, 58, 128, 165 have used SMRC in several applications to robustly obtain realizable references under restrictions both in the actuators, in the outputs, or in any state or combination of states.

The sliding control loop appears in SMRC schemes as an additional loop that makes the reference realizable under certain constraints, instead of representing the main control loop. In that way, in contrast with conventional variable structure controllers and sliding modes, the sliding regime is intended as a transitional mode of operation.

Figure 4.2 shows a generic implementation of an SMRC loop. It basically consists of two elements: a switching logic driving the search so as to fulfill the constraints and force the system to remain in the invariance set, and a filter $F$ which purpose is to smooth out the conditioned signal $r_{f}(t)$. Notice that the block $\Theta$ in the figure may represent a control loop, in which case $r$ is the 


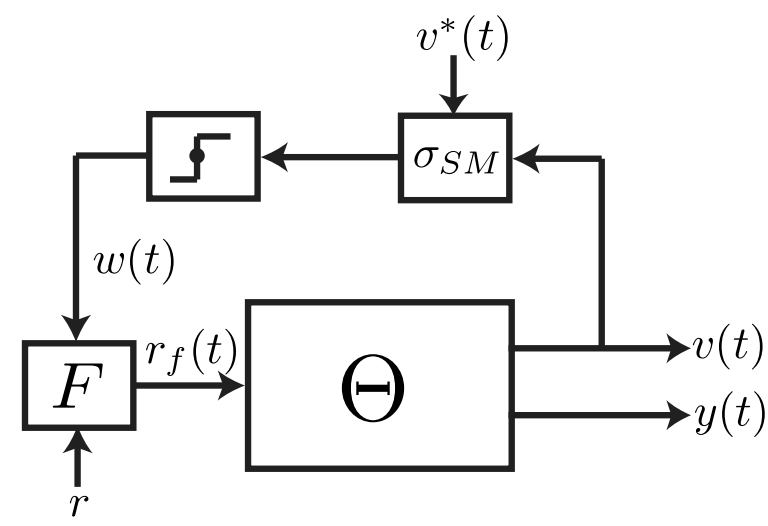

Figure 4.2: Generic scheme of an SMRC loop.

set-point while in system (4.1), $x(t)$ is the extended state vector comprising the process, controller, and filter states.

The switching logic is implemented as:

$$
w(t)=\left\{\begin{array}{lll}
w^{+} & \text {if } & \sigma_{S M}(t)>0 \\
0 & \text { if } & \sigma_{S M}(t) \leqslant 0
\end{array}\right.
$$

where

$$
\sigma_{S M}(t)=v(t)-v^{*}(t)+\sum_{i=1}^{l-1} \tau_{i}\left(v(t)^{(i)}-v^{*}(t)^{(i)}\right)
$$

with $l$ being the relative degree between the output $v(t)$ and the input $w(t)$. $v(t)^{(i)}$ and $v^{*}(t)^{(i)}$ are the $i-t h$ derivative of $v(t)$ and $v^{*}(t)$ respectively. $\tau_{i}$ are constant gains and $w^{+}$is the $w(t)$ upper value. The filter $F$ is implemented as the first-order filter $\dot{r}_{f}(t)=-\alpha\left(r_{f}(t)+w(t)-r\right)$ with $\alpha$ a design parameter.

According to the definition of the switching function in equation 4.7, it always has relative degree unitary with respect to $w(t)$ (its first derivative explicitly depends on $w(t)$ ), which is a necessary condition for SM establishment known as the transversality condition [45]. Indeed, it is interesting to observe that for a general system as the one described in system (4.1), this condition coincides with the existence condition of invariant control, $L_{g} \sigma(t) \neq 0$ (see 
Closed loop strategy: A reference conditioning method for the reduction of hypoglycemia

equation 4.4). Then, as long as $w^{+} \geqslant w^{\sigma}$-recall equations 4.5-, the SMRC loop leads to a sliding regime on $\sigma_{S M}(t)=0$ whenever the variable $v(t)$ is about to violate its constraint $v^{*}(t)$, robustly ensuring the invariance of $\Sigma$.

Note that if the initial condition is beyond the frontier defined by $\sigma_{S M}(t)=0$, the switching logic of equation 4.6 sets a control action $w^{+}$ which drives the system to the invariant region in finite time. The same would happen if an abrupt (and not sufficiently bounded) disturbance led the system to transiently leave the allowed region.

\subsection{Safety auxiliary feedback element (SAFE) in diabetes control}

In this section, the theoretical framework presented in Section 4.2 is applied to develop a safety algorithm for glucose control loops with the aim of reducing the number and severity of hypoglycemia events and to avoid late postprandial glucose rebounds. The main approach here is to limit the concentration of residual insulin in the subcutaneous tissue, the so-called IOB, whose excess is the main cause of late hypoglycemia due to delayed absorption and action.

\subsubsection{Basics of the algorithm}

Assume now that the block $\Theta$ in Figure 4.2 stands for a glucose control loop, in which the main controlled variable $y(t)$ is the glucose concentration $(G(t))$ and the constrained variable $v(t)$ is the $\operatorname{IOB}(\mathrm{t})$ (henceforth $\mathrm{IOB}$ ).

A representation of the proposed closed-loop scheme is shown in Figure 4.3. $C$ represents a controller. The control action is the pump's insulin infusion rate $\left(u_{c}(t)\right)$, while $u_{f}(t)$ represents a priming bolus in meal announcement schemes. For the subsequent analysis, the inner controller $C$ is assumed to be a realizable proportional-integral-derivative (PID) controller. However, it is worth remarking that it could be any other controller, even nonlinear and strictly-proper ones. 


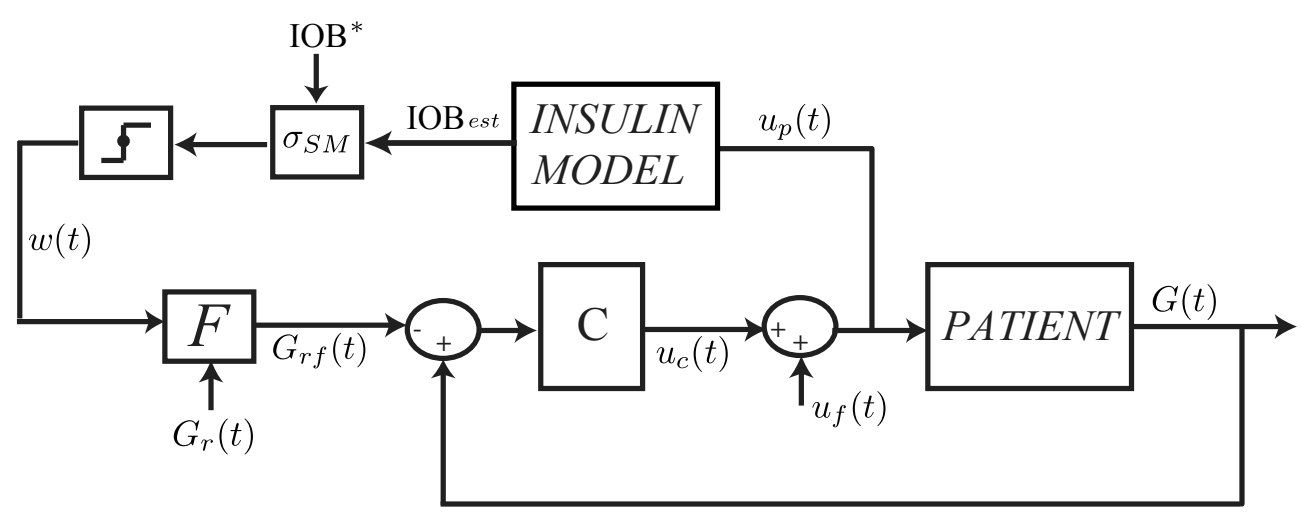

Figure 4.3: SMRC implementation for diabetes application.

Hence,

$$
u_{c}(t)=k_{P} e(t)+k_{D} \dot{G}+k_{I} \int_{0}^{t} e(\tau) \mathrm{d} t
$$

where $e(t)=G(t)-G_{r f}(t)$ with $G_{r f}(t)$ the conditioned reference further defined in equation (4.11) and $k_{P}, k_{D}$ and $k_{I}$ the constants of the proportional, derivative, and integral parts of the PID respectively. Notice that when $u_{c}(t)$ (insulin injected) increases, the glucose concentration $G(t)$ decreases. This fact explains the error sign.

Since the IOB is inaccessible, it must be estimated. Again, the proposed methodology does not restrict the way in which IOB is estimated, which can be performed by means of any of the published insulin absorption models [85, 91, 98, 129, 149, 157, 162, 181, 16, 178, 100, or even employing static pharmacokinetic curves as currently done in commercially available insulin pumps. Here, the insulin absorption model developed by Cobelli's group [100] and presented in Section 1.4.2 is used for the method description (Figure 4.4). Its equations for the subcutaneous insulin absorption take the form:

$$
\begin{aligned}
& \dot{S}_{1}(t)=-\left(k_{a 1}+k_{d}\right) S_{1}(t)+u_{p}(t) \\
& \dot{S}_{2}(t)=k_{d} S_{1}(t)-k_{a 2} S_{2}(t)
\end{aligned}
$$

where $S_{1}(t)$ and $S_{2}(t)$ are subcutaneous tissue compartments (insulin mass), 
Closed loop strategy: A reference conditioning method for the reduction of hypoglycemia

$u_{p}(t)$ represents the administration of insulin, $k_{a 1}, k_{a 2}$ are rate constants of insulin absorption and $k_{d}$ is the inter-compartment transport rate.

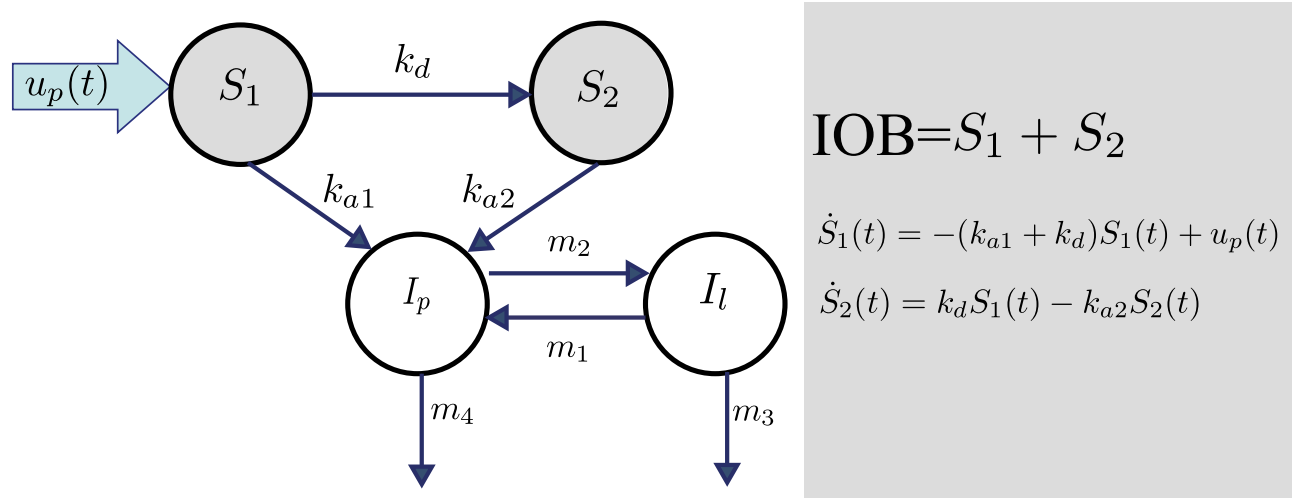

Figure 4.4: Schematic representation and equations of Cobelli's insulin model used here to estimate IOB.

In this way, IOB is defined as:

$$
\mathrm{IOB}=S_{1}(t)+S_{2}(t)
$$

As mentioned in the previous section, the outer SMRC loop consists of two main elements. A switching logic responsible for constraining IOB inside the desired bounds, and a first-order filter $F$ which purpose is to smooth out the conditioned reference.

The first-order filter takes the form:

$$
\dot{G}_{r f}(t)=-\alpha\left(G_{r f}(t)+w(t)-G_{r}\right)
$$

where $G_{r}$ (usually constant) is the desired reference when IOB does not reach the defined bounds, $G_{r f}(t)$ is the conditioned reference, $w(t)$ is the injected discontinuous signal and $\alpha$ determines the filter cut-off frequency.

Two constraints for the IOB can be defined. One upper constraint $(\overline{\mathrm{IOB}(t)})$ (from here on $\overline{\mathrm{IOB}}$ ) to mitigate, as explained before, the problem of hypoglycemia incidence, and an additional lower constraint $(\underline{\mathrm{IOB}(t)})$ (from 


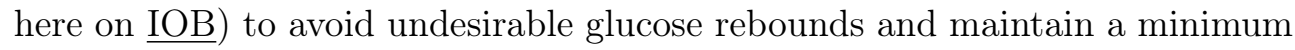
quantity of IOB. To design the corresponding sliding functions, it is necessary to know the relative degree $l$ between IOB and $w(t)$. Defining IOB as $S_{1}(t)+S_{2}(t)$, this relative degree is $l=2$. Hence, according to (4.7), the sliding functions are defined as 1

$$
\begin{aligned}
\sigma_{1}(t) & =\mathrm{IOB}-\overline{\mathrm{IOB}}+\tau(\mathrm{IO} B-\dot{\mathrm{IOB}})= \\
& =\left(1-\tau k_{a 1}\right) S_{1}(t)+\left(1-\tau k_{a 2}\right) S_{2}(t)+\tau u_{p}(t)-\overline{\mathrm{IOB}}-\tau \dot{\overline{\mathrm{IOB}}} \\
\sigma_{2}(t) & =\mathrm{IOB}-\underline{\mathrm{IOB}}+\tau(\dot{\mathrm{IOB}}-\underline{\mathrm{IOB}})= \\
& =\left(1-\tau k_{a 1}\right) S_{1}(t)+\left(1-\tau k_{a 2}\right) S_{2}(t)+\tau u_{p}(t)-\underline{\mathrm{IOB}}-\tau \underline{\mathrm{IOB}}
\end{aligned}
$$

and the associated switching logic is:

$$
w(t)=\left\{\begin{array}{c}
w^{+} \text {if } \sigma_{1}(t)>0 \\
w^{-} \text {if } \sigma_{2}(t)<0 \\
0 \text { otherwise }
\end{array}\right.
$$

Note that, because of the way the system is defined, $w^{+}$is negative and $w^{-}$positive. That is, when the upper bound is violated, the reference value is increased so as to reduce the control action, and vice versa for the lower constraint. In other words, when $\sigma_{1}(t)>0$, IOB is higher than $\overline{\mathrm{IOB}}$. In order to decrease IOB, the insulin injected $\left(u_{c}(t)\right)$ must decrease. This effect is achieved increasing $G_{r f}(t)$, avoiding hypoglycemia due to an excess of insulin. In the same way, when $\sigma_{2}(t)<0$, IOB is lower than $\underline{\text { IOB }}$ and $G_{r f}(t)$ must decrease in order to force an increase in $u(t)$ and inject more insulin. This procedure avoids undesirable later glucose rebounds once the effect of the meal has already been counteracted.

As the algorithm is thought to be added to any closed-loop controller in

\footnotetext{
${ }^{1}$ Note that the compensation design is exactly the same independently of the controller and estimator -provided their relative degree is the same, which is the case in practice as otherwise additional lag would be unnecessarily introduced to the control loop-.
} 
Closed loop strategy: A reference conditioning method for the reduction of hypoglycemia

order to provide an additional safety layer, it is named here Safety Auxiliary Feedback Element (SAFE).

Remark: It is well known that the main drawback of sliding mode control is the chattering phenomena, but in the proposed SAFE algorithm this problem is not present. On the one hand, the discontinuous action is not a physical signal due to the software-based nature of the algorithm. On the other hand, it is filtered previously to being used to modify the reference. Therefore, all signals in the main control loop are smooth.

\subsubsection{Robustness and fault-tolerance properties}

One of the most relevant features of sliding modes is their robustness against an important sort of uncertainties and disturbances, the so-called matched perturbations. Indeed, the matching condition of sliding regimes [45] states that the dynamics during SM is insensitive (which is more than robustness) to any bounded disturbance being collinear with the discontinuous action.

For the SAFE algorithm, combining the expressions related to IOB of the insulin system (equations (4.9)), the controller (equation (4.8)) and the filter (equation (4.11)), the open-loop dynamics of the whole conditioning system can be rewritten in the general form of system 4.1) as:

$$
\begin{aligned}
\left(\begin{array}{c}
\dot{S}_{1}(t) \\
\dot{S}_{2}(t) \\
\dot{u}_{p}(t)
\end{array}\right) & =\left(\begin{array}{c}
\left(-\mathrm{k}_{\mathrm{a} 1}-\mathrm{k}_{\mathrm{d}}\right) S_{1}(t)+u_{p}(t) \\
k_{d} S_{1}(t)-k_{a 2} S_{2}(t) \\
-u_{p}(t)
\end{array}\right)+\left(\begin{array}{c}
0 \\
0 \\
\psi
\end{array}\right)+\left(\begin{array}{c}
0 \\
0 \\
k_{P} \alpha
\end{array}\right) w(t) \\
\psi= & -k_{P} \alpha G_{r}-\left(k_{I}-k_{P} \alpha+k_{P}\right) G_{r f}(t)+\left(k_{P}+k_{I}\right) G(t)+ \\
& +\left(k_{P}+k_{D}\right) \dot{G}(t)+k_{D} \ddot{G}(t)+k_{I} \int_{0}^{t} G(t) \mathrm{d} t- \\
& -k_{I} \int_{0}^{t} G_{r f}(t) \mathrm{d} t+u_{f}(t)+\dot{u}_{f}(t)
\end{aligned}
$$


where, following the general notation of system 4.1):

$$
x(t)=\left(\begin{array}{c}
S_{1}(t) \\
S_{2}(t) \\
u_{p}(t)
\end{array}\right), g(x)=\left(\begin{array}{c}
0 \\
0 \\
k_{P} \alpha
\end{array}\right),
$$

and $f(x, d)$ is composed of the first two terms of the right-hand-side of system 4.14.

The above equation shows that the second term, which can be seen as the perturbation vector $d(t)$, is collinear with the control vector $g(x)$ which determines the direction of the discontinuous action, i.e., they satisfy the matching condition. Thus, once SM is established on surfaces $\sigma_{1}(t)=0$ or $\sigma_{2}(t)=0$, the resulting SM dynamics is insensitive to changes in $G_{r}, G(t)$ and $u_{f}(t)$. This means that the limits imposed to IOB are robust against set-point changes, measurement noise and over-estimated priming bolus doses, and avoid also high concentrations of residual insulin due to sensor failures. Note that this robustness is referred to the IOB limits and does not imply a delay in the inner controller reaction to those changes.

Observe that although $\dot{G}(t), \ddot{G}(t)$ and $\dot{u}_{f}(t)$ appear in $d(t)$, they do not affect the robustness of the algorithm as they could only be unbounded during given time instants (e.g., at glucose monitor samplings or start of bolus doses), after which the commanded signal to the pump will be consistent with the IOB constraints. Recall that the software-based SAFE algorithm can operate at the much faster rates than the main control loop, which guarantees that, in case IOB is going to violate the constraints in the period between sensor measurements, the algorithm detects this violation. In this way, the algorithm continuously calculates the insulin needed for keeping the IOB under the constraint between samples and, at the next sampling rate of the insulin pump it can inject, for example, the mean value of all the calculations. Moreover, $\ddot{G}(t)$ does only appear if we consider a controller with a pure differentiator, something improbable in practice, and $\dot{u}_{f}(t)$ can always be bounded by means of a fast filtering (imperceptible for the slow open-loop system dynamics) of the feed-forward action. 
Closed loop strategy: A reference conditioning method for the reduction of hypoglycemia

\subsubsection{SM establishment on safety $I O B$ constraints}

From Section 4.2 , the necessary condition that must be fulfilled so as the sliding mode to exist is the transversality condition, that is $L_{g} \sigma(t)=\frac{\partial \sigma(t)}{\partial x(t)} g(x) \neq 0$. As $L_{g} \sigma_{1}(t)=L_{g} \sigma_{2}(t)$, calculations are carried out in the following for $\sigma_{1}(t)$ being the procedure for $\sigma_{2}(t)$ analogous:

$$
L_{g} \sigma_{1}(t)=\frac{\partial \sigma_{1}(\mathrm{t})}{\partial \mathrm{x}}(\mathrm{t}) g(x)=\left(\begin{array}{ccc}
1-\tau k_{a 1} & 1-\tau k_{a 2} & \tau
\end{array}\right)\left(\begin{array}{c}
0 \\
0 \\
k_{P} \alpha
\end{array}\right)=\tau k_{P} \alpha
$$

where $k_{P}, \tau$ and $\alpha$ are design parameters, always different from 0 and positive. Therefore, the transversality condition holds.

Since the objective here is to shape the reference signal $G_{r f}(t)$, as a rule of thumb it is reasonable to take $w^{+}$of the order of the glucose set-point $G_{r}$. Nevertheless, the exact minimum amplitude to guarantee SM can be explicitly computed from the invariance condition stated in Section 4.2 .

$$
\left(w^{+}-w^{\sigma_{1}}\right) L_{g} \sigma_{1}(t) \leqslant 0, \forall x(t) \in \partial \Sigma
$$

According to equations 4.5, since in this case $L_{g} \sigma_{1}(t)>0, w^{+}$must be chosen to fit equation:

$$
w^{+} \leqslant w^{\sigma_{1}}=-\left(L_{g} \sigma_{1}(t)\right)^{-1}\left[L_{f} \sigma_{1}(t)+\dot{\overline{\mathrm{IOB}}}\right]=\xi(x, \tau, \alpha)<0
$$

with $\xi(x, \tau, \alpha)$, derivable from equations 4.14 and 4.18), defining the minimum amplitude for the negative value $w^{+}$so as to guarantee SM.

\subsubsection{IOB constraints definition}

One of the critical points to guarantee the reduction of the hypoglycemia events using SAFE is the selection of the proper IOB constraints. This decision can 
be based on different criteria and it differs from one individual to another. Indeed, the most suitable IOB constraints depend on the insulin sensitivity of each patient, together with the meal amount and composition ingested.

Different parameters can be used as insulin sensitivity estimation. Demographic (age) and anthropometric parameters such as the body mass index (relation between height and weight), the body fat (fat percentage of body weight) and the waist circumference [18, 167] together with metabolic parameters as total daily dose of insulin, the insulin-to-carbohydrate ratio, correction factor and basal rate prescribed by the physician (insulin pump tuning), can be combined to perform the estimation. Remark also the time-varying nature of insulin sensitivity due, among others, to the circadian rhythms [65] that can be taken into account in the definition of the IOB constraints, making them time-variable along the day. In order to avoid the uncertainty caused by the inaccurate estimation of the carbohydrate content of a meal by the patients, meal size could be estimated using the measured glucose slope, but the high variability observed in glucose absorption advises against this approach. Finally, physician's knowledge and experience always play an important role in the selection of the proper IOB constraints for each patient. Note also that the bounds $\overline{\mathrm{IOB}}$ and $\underline{\mathrm{IOB}}$ can initially be chosen in a conservative way so as to compensate for uncertainty in the insulin absorption model (see equations (4.9)), and then they could be easily adjusted by the physicians to each patient.

One approach to define $\overline{\mathrm{IOB}}$ limit is to adjust it to a piecewise function as illustrated in Figure 4.5. It consists of a security constraint during the night period, a higher constraint (meal dependent) for the postprandial period and a more restrictive constraint for the late postprandial period specially useful in meals with high fat content. Depending on the scenario, other option could be to fix just one daily (conservative) and one nightly security constraint in order to obtain a non-meal dependent outer loop to be used in fully automated control loops. An additional lower constraint can be added to avoid glucose rebounds. A good way to define this lower constraint is to build it proportional to the usual basal rate of the patient, that could be time-variable in case of pump therapies. 
Closed loop strategy: A reference conditioning method for the reduction of hypoglycemia

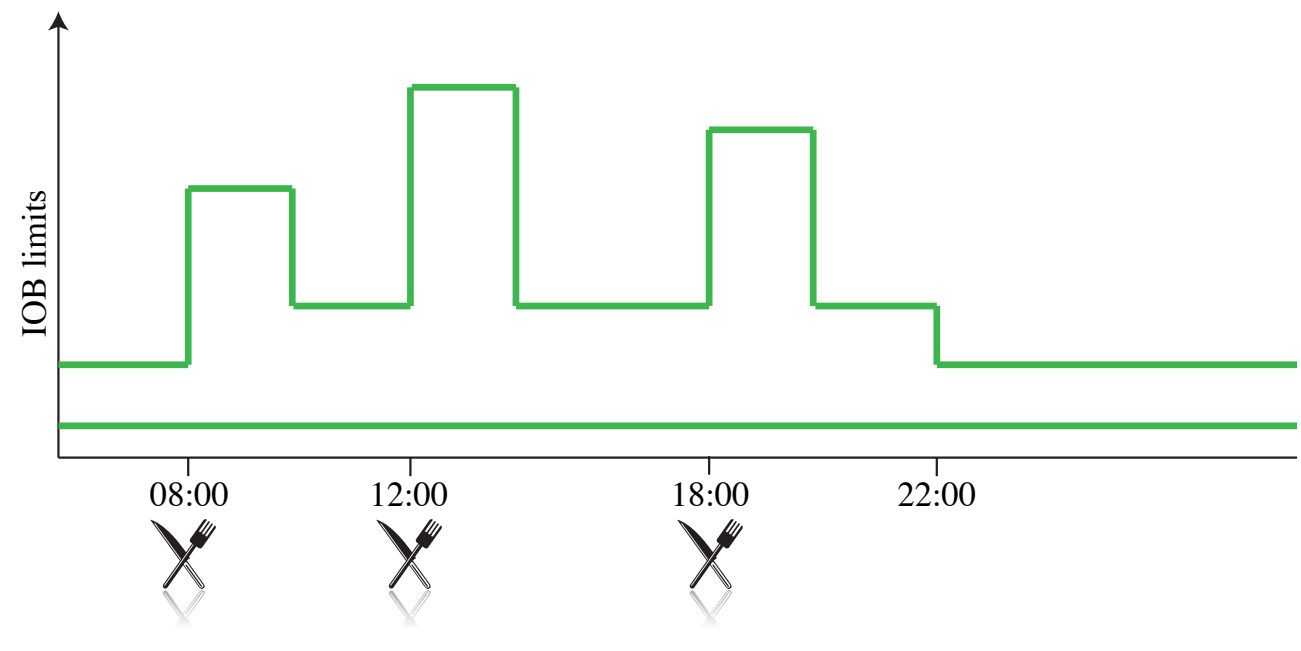

Figure 4.5: Daily IOB constraint function.

Note that although IOB limits can be time-variant $\left(v^{*}(t)\right.$ in Section 4.2.1), their variation must be slower than the bandwidth of SAFE. Thus, the case of IOB limits piecewise constant should be addressed in practice with ramp-like changes instead of steps. In any case, the dynamics of the changes could still be much faster than patient dynamics (e.g., 5 min. ramps) but slow enough for SAFE.

In this thesis, the size of the meal together with the total daily dose of insulin (TDD) as an estimator of the insulin sensitivity are used for the definition of the postprandial IOB limits in the in silico evaluation of the methodology (Section 4.4.2). The time-varying nature of the insulin sensitivity has not been taken into account in this step in order not to complicate the tuning of the IOB limits definition. The lower IOB constrained has been defined proportional to the basal rate (in this case the same during the whole day) of each patient. However, any other approximation to define IOB limits could be used. 


\subsection{Simulations and results}

The evaluation of the above explained methodology has been carried out through an in silico study using the UVA simulator [88]. Previously, some simulations are presented to illustrate the robustness of the methodology, demonstrated theoretically in Section 4.3.2.

\subsubsection{SAFE algorithm simulations illustrating robustness}

As an illustration of the SAFE principle, a one-day simulation (40-80-70g) is shown in Figure 4.6 comparing performance of a PID with and without SAFE. A discontinuous signal $(w(t))$ driving the PID set-point (after filtering) is generated when the IOB is in the upper (lower) limit imposed by the constraints. When IOB remains inside the allowed region, the discontinuous signal is zero. For this simulation and the followings, the initial set-point of the inner controller $\left(G_{r}\right.$ in equation 4.11) $)$ is fixed to $100 \mathrm{mg} / \mathrm{dl}$. Note that, in this particular example, the lower IOB limit allows a reduction in the glucose peaks, whereas the upper limit reduces hypoglycemia risk. It is worth mentioning that the lower IOB bound does not prevent the controller from suggesting insulin delivery to zero, although the time the insulin delivery is zero is reduced. The objective of this constraint is to avoid a situation of total absence of insulin $(\mathrm{IOB}=0)$.

In Figure 4.7 the robust behavior of the proposed algorithm is demonstrated through 3 simulations that represent typical failures of CGMS. Solid lines represent the sensor measurements with their corresponding failures and dotted lines the actual glucose profile. Red and blue lines correspond to the performance with and without SAFE against the same sensor failures. Case 1 represents a sensor drift where the upper deviation from the actual glucose value is forced to be abrupt to illustrate the worst-case scenario. The calibration point at time $=200$ min makes the sensor return to the correct value. The controller reaction to a high increase in glucose concentration is to apply more insulin, over-reacting with the subsequent hypoglycemia risk. Constraints in IOB reduce this over-reaction avoiding risk situations. Case 
Closed loop strategy: A reference conditioning method for the reduction of hypoglycemia
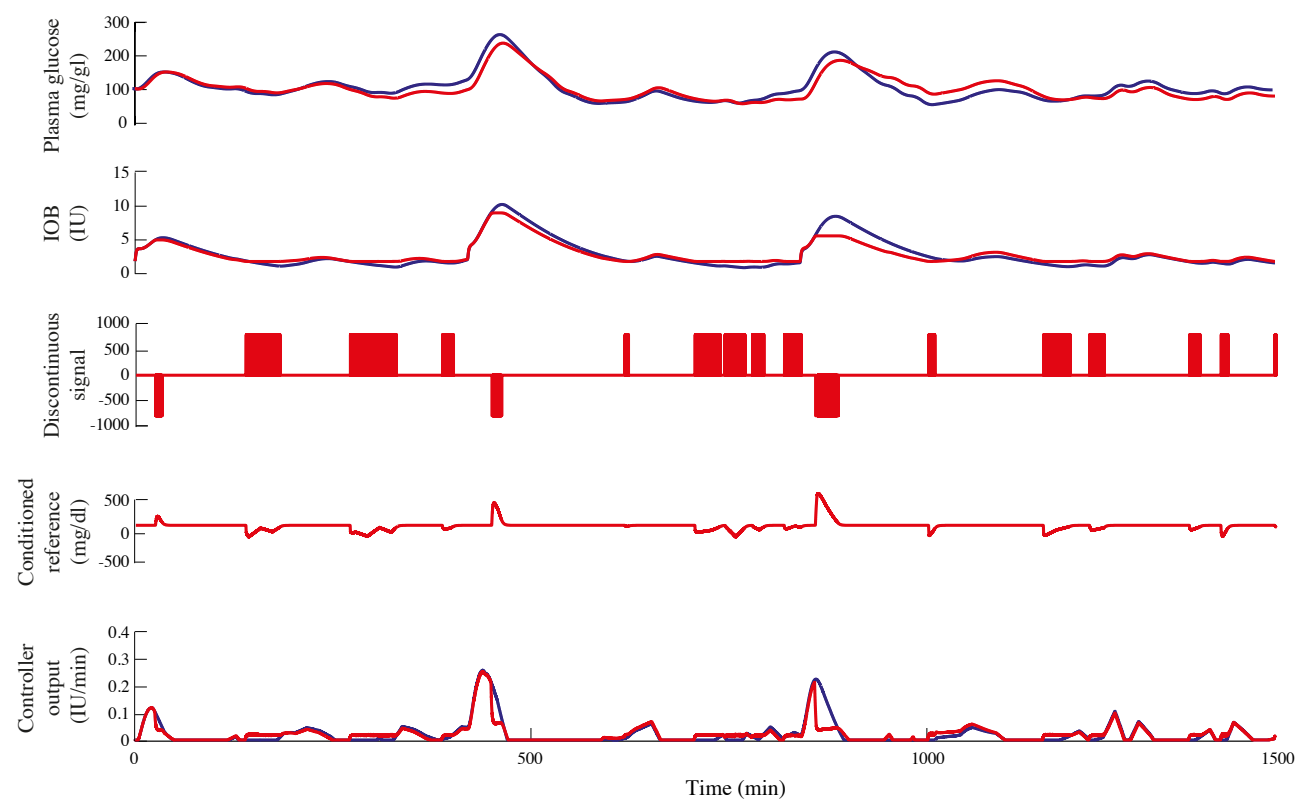

PID without SAFE

PID with SAFE

Figure 4.6: Comparison of the performance of the controller in one virtual patient (patient 4) with a conventional PID and with a PID plus the additional SAFE loop during one day. The glucose profile, together with the IOB profile, and the discontinuous signal with the realizable reference derived from it are presented. Additionally, the actual response of the controller with and without SAFE is also shown. 
2 shows a similar situation where the sensor drifts until saturation at the lower limit of a typical CGMS $(40 \mathrm{mg} / \mathrm{dl})$. In this case, SAFE prevents the hyperglycemia after the sensor drift. Case 3 represents a common situation of signal loss, that also can cause later hyperglycemia events.
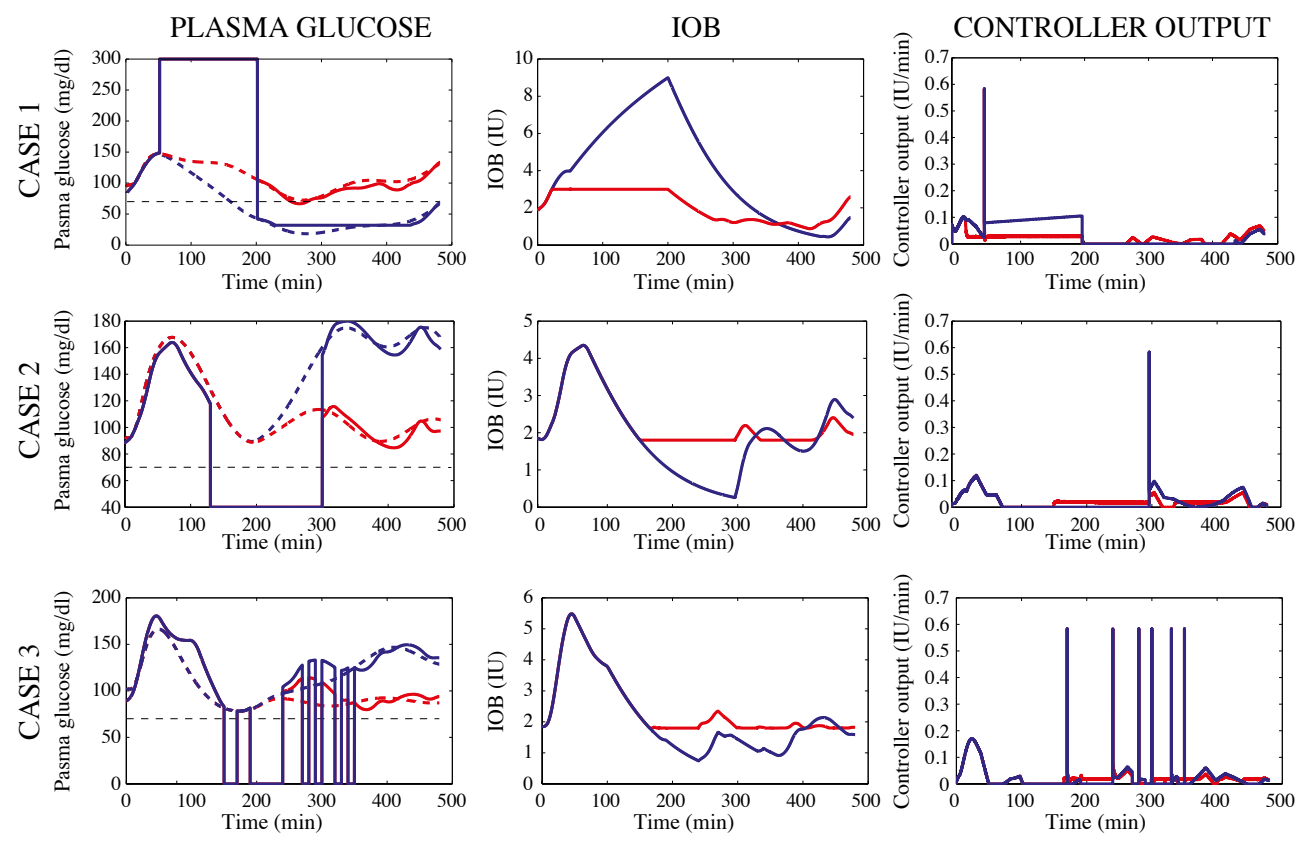

PID without SAFE

PID with SAFE

Figure 4.7: Glucose response,IOB profile and controller response (no priming bolus is considered here) in the presence of sensor failures. Blue lines represent the response without SAFE loop whereas red lines correspond to the response using SAFE. In the glucose profiles, solid lines are the sensor signal and dotted lines the actual glucose profile.

These sensor failures can produce undesired responses of the controller leading to late hypo or hyperglycemia events. By adding the SAFE loop with the upper and lower constraints for IOB, this effect is avoided, showing the robustness of the algorithm against sensor failures as it was mathematically demonstrated in Section 4.3.2. In the same way, SAFE algorithm is also robust against other type of perturbations, as for example an over-estimation 
Closed loop strategy: A reference conditioning method for the reduction of hypoglycemia

of the priming bolus usually used to compensate for meals. This effect is shown in Figure 4.8 where for a $50 \mathrm{~g}$ meal, a $4 I U$ bolus $(30 \%$ higher than the usual patient's therapy) is administered as feed-forward bolus. The SMRC loop detects too high IOB values and it is capable of keeping IOB below its constraint, provided that the amplitude of $w^{+}$designed in 4.18 is high enough. If that was not the case, the IOB limit could be temporally violated but SAFE would contribute to enter again in the allowed region with $w(t)=w^{+}$ (see Section 4.2.2). That situation is illustrated in Figure 4.9. In both cases, the insulin given by the inner controller is reduced and therefore hypoglycemia is avoided. This simulation has been repeated for all the 10 available virtual adult patients and the area under the curve (AUC) of plasma glucose (PG) below $70 \mathrm{mg} / \mathrm{dl}$ with and without SAFE has been represented in Figure 4.10. It shows that the AUC with SAFE is always lower than without it, being the time spent below $70 \mathrm{mg} / \mathrm{dl}$ zero in most cases.
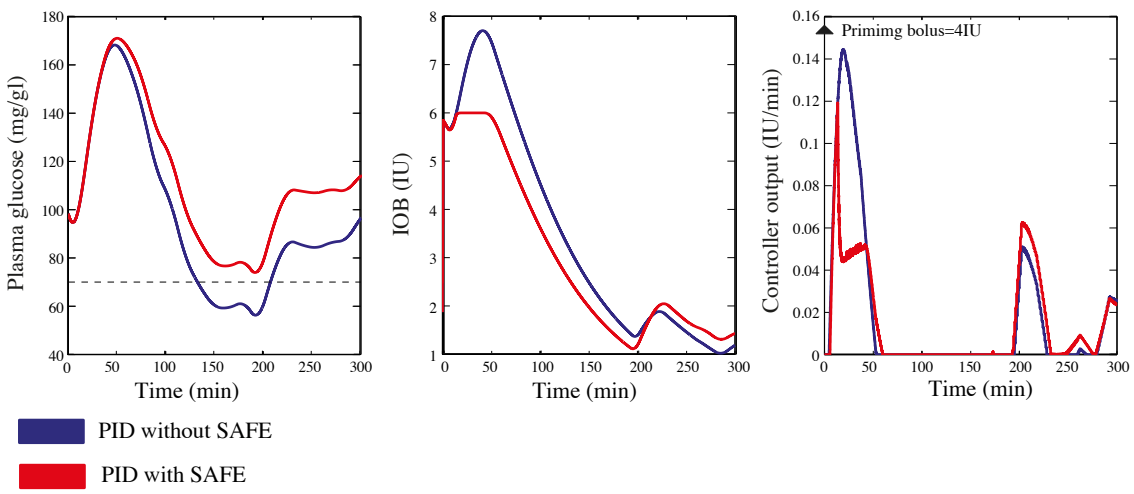

Figure 4.8: Plot that illustrates the SAFE reaction against over-estimated priming bolus. In this case, a $4 \mathrm{IU}$ bolus is added to the control output for a small meal $(50 \mathrm{~g})$. The third figure represents the controller output $u_{c}(t)$ in Figure 4.3. The actual insulin delivery is $u_{p}(t)=u_{c}(t)+u_{f}(t)$ with $u_{f}(t)$ being the priming bolus.

\subsubsection{In-silico evaluation using UVA simulator}

Finally, an in silico study using the cohort of 10 adult virtual patients available in the educational version of the UVA simulator [88] is presented below. Those 

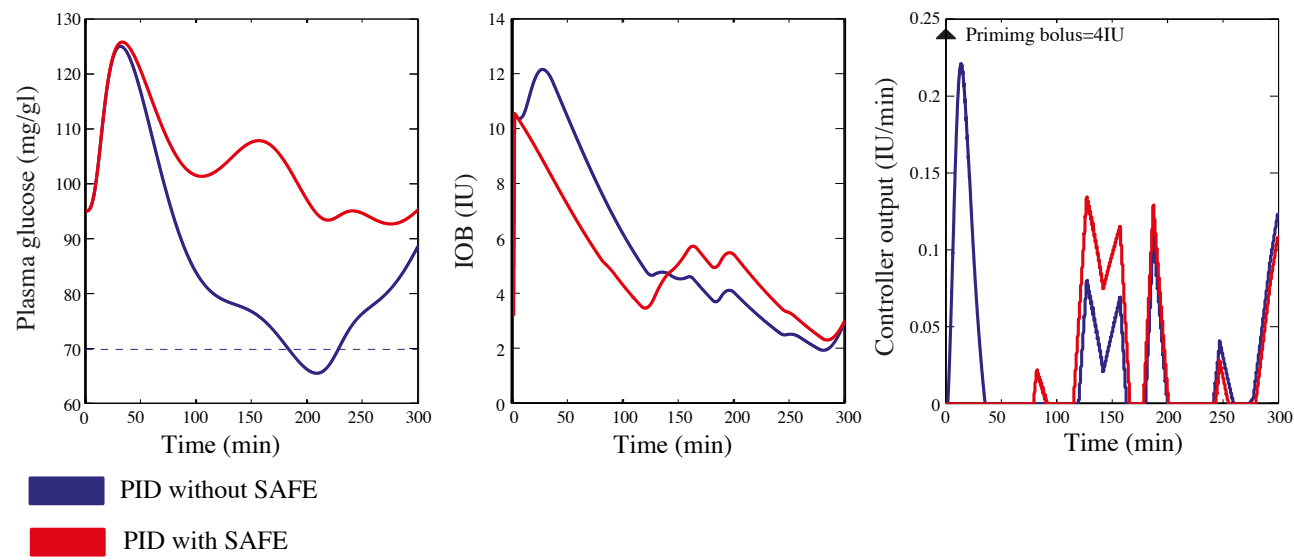

Figure 4.9: Plot that illustrates the SAFE reaction against over-estimated priming bolus in case $w^{+}$has not been designed high enough. The IOB limit is temporally violated but SAFE always contributes to enter again in the allowed region with $w(t)=w^{+}$.

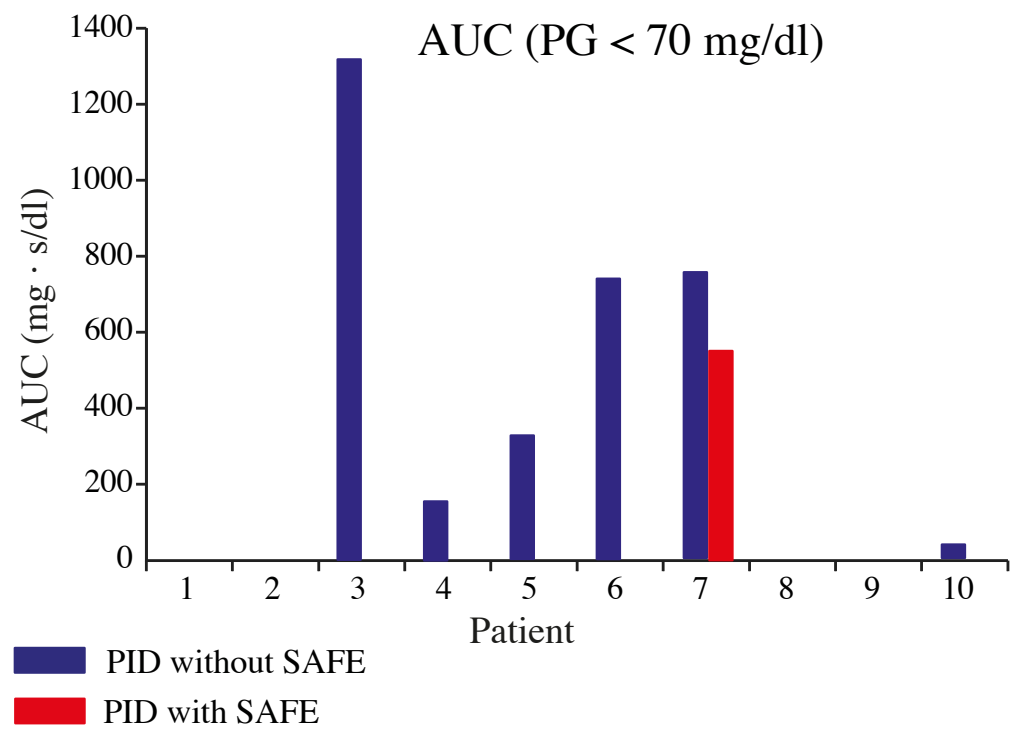

Figure 4.10: Plot that shows the AUC of $P G<70 \mathrm{mg} / \mathrm{dl}$ for the 10 available virtual bolus for a $50 \mathrm{~g}$ meal and a feed-forward bolus $30 \%$ higher than the usual. 
Closed loop strategy: A reference conditioning method for the reduction of hypoglycemia

Table 4.1: PID tuning for each of the patient

\begin{tabular}{lccccccccccc}
\hline & Pat. 1 & Pat. 2 & Pat. 3 & Pat. 4 & Pat. 5 & Pat. 6 & Pat. 7 & Pat. 8 & Pat. 9 & Pat. 10 \\
\hline $\mathbf{k}_{\mathbf{P}}(I U / h * d l / m g)$ & 0.063 & 0.077 & 0.056 & 0.023 & 0.085 & 0.033 & 0.038 & 0.058 & 0.041 & 0.058 \\
$\mathbf{k}_{\mathbf{D}}(I U * d l / m g)$ & 7.603 & 9.216 & 6.735 & 2.816 & 10.240 & 3.980 & 4.608 & 6.912 & 4.896 & 6.912 \\
$\mathbf{k}_{\mathbf{I}}\left(I U / h^{2} * d l / m g\right)$ & $0.14 \mathrm{e}-3$ & $0.17 \mathrm{e}-3$ & $0.12 \mathrm{e}-3$ & $0.05 \mathrm{e}-3$ & $0.19 \mathrm{e}-3$ & $0.07 \mathrm{e}-3$ & $0.08 \mathrm{e}-3$ & $0.13 \mathrm{e}-3$ & $0.91 \mathrm{e}-4$ & $0.13 \mathrm{e}-3$ \\
\hline
\end{tabular}

virtual patients are the same used in the evaluation of the open-loop algorithm presented in Chapter 3 . Their demographic, anthropometric, and metabolic parameters can be found in Table 3.1 .

A 16-hour clinical protocol corresponding to active daily hours (from $8 \mathrm{~h}$ to $24 \mathrm{~h}$ ) of three meals (8:00 am, noon, and 6:00 pm) of 40, 80, and 70 $\mathrm{g}$ during 10 days was considered. In order to test the robustness of the methodology with respect to intra-patient variability, sinusoidal oscillations of $5 \%$ amplitude (except for insulin sensitivity which was 10\%) and $3 \mathrm{~h}$ period were superimposed on nominal values of the model parameters in a similar way as in [177. A PID controller tuned individually for each patient was used as main controller.

A subcutaneous glucose sensor model was also included to account for noisy measurements. Performance of the controller with and without SAFE was compared.

The specific PID parameters used for each patient are shown in Table 4.1. They have been determined, taking advanced of the in silico nature of the evaluation, through simulations using plasma glucose measures. In real practice the parameters of the controller could be determined using the information of the usual pump therapy of the patient in a similar way as in [153]. Due to its proved superiority against fully closed-loop systems a feedforward meal announcement was added to the control scheme. In this case, a fixed 2IU bolus was infused at mealtime as in [114].

In order to deal with inter-patient variability, different postprandial upper limits for IOB were defined depending on an estimation of the insulin sensitivity of each patient. This estimation was carried out computing TDD using the 
Table 4.2: Upper postprandial $\overline{\mathrm{IOB}}$ limits

\begin{tabular}{lcccc}
\hline & $T D D<=60$ & $60<T D D<75$ & $75<T D D<85$ & $T D D>85$ \\
\hline$C H O<40 g$ & 3 & 3 & 3 & 3 \\
$40 g<C H O<70 g$ & 5 & 7 & 9 & 10 \\
$70 g<C H O<100 g$ & 7 & 9 & 11 & 13 \\
$C H O>100 g$ & 9 & 10 & 13 & 15 \\
\hline
\end{tabular}

basal insulin rate of each patient, i.e. , basal infusion normalizing plasma glucose $(\mathrm{PG})$ around $100 \mathrm{mg} / \mathrm{dl}(\mathrm{TDD}=$ basal[IU/h]242). The IOB limit depended also on the meal size, allowing higher IOB values for big meals and being more restrictive with small meals. Table 4.2 shows the specific limits that have been used for this evaluation.

Additionally, these limits were reduced if they were caused by the second peak of the meal absorption (later postprandial period) or if the patients initial condition was moderate hypoglycemia ( $10 \%$ decrease). Moreover, initial conditions of hyperglycemia allowed higher values of IOB (10\% increase). In order to maintain always a minimum of IOB to keep a good basal glucose concentration and avoid glucose rebounds, a fixed lower IOB bound for each patient (proportional to their basal rate) was established for all the situations.

In Table 4.3 a set of indicators are provided comparing the performance of a controller with and without SAFE. The number of potentially severe $(P G<50$ $\mathrm{mg} / \mathrm{dl}$ ) and moderate hypoglycemia $(50 \mathrm{mg} / \mathrm{dl}<P G<70 \mathrm{mg} / \mathrm{dl}$ ) events together with the percentage of time in these values are provided. Additionally, the percentage of time above $180 \mathrm{mg} / \mathrm{dl}$ and between $140 \mathrm{mg} / \mathrm{dl}$ and $180 \mathrm{mg} / \mathrm{dl}$ is also provided. Normoglycemia is here defined as $70 \mathrm{mg} / \mathrm{dl}<P G<140$ $\mathrm{mg} / \mathrm{dl}$. All data were subjected to repeated-measures analysis of variance with Huynh-Feldt adjustment for nonsphericity (the p-value of each comparison is also included in Table 4.3] [180]. Note that the parameters used in equation (4.9) to estimate IOB are population parameters, so the results presented 
Closed loop strategy: A reference conditioning method for the reduction of hypoglycemia

Table 4.3: Different indicators for the evaluation of SAFE

\begin{tabular}{lccccccc}
\hline & $P G<50(\%)$ & $50<P G<70(\%)$ & $P G>180(\%)$ & $140<P G<180(\%)$ & Normo(\%) & $\begin{array}{c}\text { Severe } \\
\text { hypo events }\end{array}$ & $\begin{array}{c}\text { Moderate } \\
\text { hypo events }\end{array}$ \\
\hline Without SAFE & 3.96 & 11.79 & 2.64 & 9.25 & 72.34 & 44 & 97 \\
With SAFE & 0.35 & 6.64 & 3.39 & 12.52 & 77.08 & 9 & 72 \\
p value & 0.018 & 0.01 & 0.389 & 0.006 & 0.011 & 0.002 & 0.003 \\
\hline
\end{tabular}

here demonstrate also robustness with respect to discrepancies between the estimated IOB and the real one.

Potentially severe hypoglycemia events $(P G<50 \mathrm{mg} / \mathrm{dl})$ were almost avoided using SAFE (44 vs $9, p=0.002$ ) reducing the percentage of time in hypoglycemia $(P G<70 \mathrm{mg} / \mathrm{dl})$ more than $50 \%(p<0.001)$. Reduction of hypoglycemic exposure was not associated with an increase in the risk of hyperglycemia. Indeed, hyperglycemic exposure (time spent above $180 \mathrm{mg} / \mathrm{dl}$ ) was not different $(2.84 \%$ of time vs $3.39 \% p=0.389)$.

Figure 4.11 shows graphically the detailed metrics for each of the 10 patients. In most of the patients the reduction in the time in hypoglycemia does not imply a corresponding increase in hyperglycemia. In fact, in some cases, the time above $180 \mathrm{mg} / \mathrm{dl}$ is reduced with SAFE. Note that the cases where this increase exists correspond to the cases with highest reduction in potentially severe hypoglycemia, mainly due to the initial conditions of the last meal.

\subsection{Conclusions}

In this chapter, a new approach for reducing the risk of hypoglycemia, especially in the postprandial period, considered as one of the challenges for the development of an efficient artificial pancreas has been presented. The algorithm, called SAFE, is shown as a security loop to be added to the main control loop that is only active when IOB is about to violate any previously defined constraint. Otherwise, the SAFE loop is inactive and the original control system is not altered at all. In this way, the approach exploits the attributes of the sliding regime as a transitional mode of operation. 

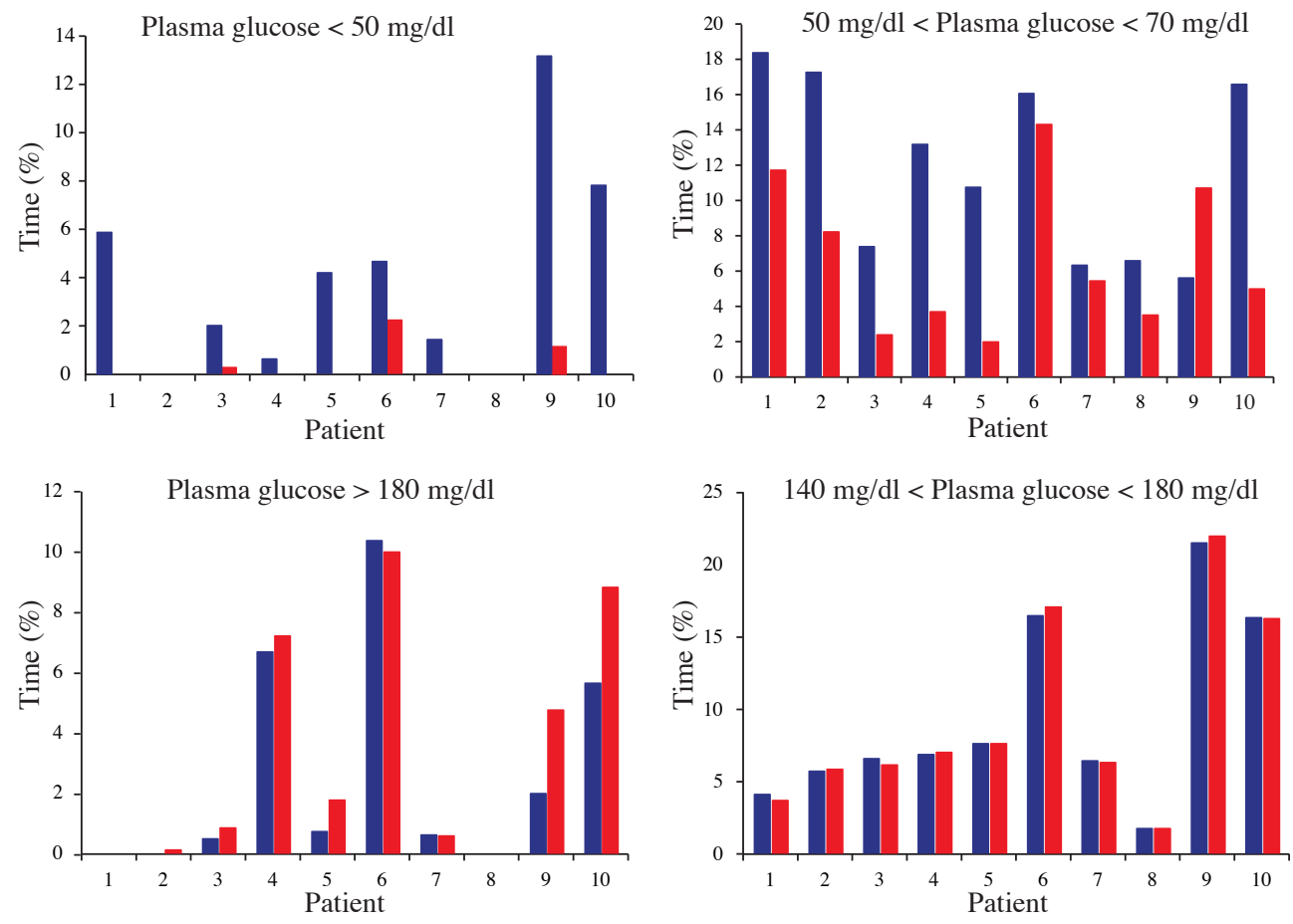

PID without SAFE

PID with SAFE

Figure 4.11: Percentage of time in hypo and hyperglycemia of each patient with and without SAFE loop. 
Closed loop strategy: A reference conditioning method for the reduction of hypoglycemia

The theoretical basis of the algorithm are detailed, demonstrating its robustness against perturbations. Moreover, an in silico evaluation with a sample of the adults' cohort of the UVA Simulator is presented. The results obtained are promising, reducing the number and the duration of hypoglycemia events, in spite of considering intra-patient variability and population parameters for the IOB estimation.

In conclusion, despite the limitations of every in silico evaluation, this is a proof of concept study that may prelude the development of clinical studies to validate SAFE algorithm. In fact clinical trials to evaluate the efficacy of the algorithm in vivo are currently on-going (NCT02100488).

Some improvements to gain in robustness against uncertainty are presented in next chapter. 



\section{$5 \quad$ Extending SAFE to improve its performance against vari- ablity}

In Chapter 4 an SMRC scheme to be added to any closed-loop glucose control to improve its performance was presented. Indeed, it demonstrated superiority with respect to the single closed-loop, together with robustness against sensor failures and over-estimations of the priming bolus. However, some improvements can be added to the algorithm so as to increase its robustness against other factors such as parametric uncertainty.

In Section 2.4.1, variability is posed as one of the challenges glucose closed loop control has to counteract. In [47, differences in the rate of insulin absorption from one patient to another is shown to be an important factor for the controller design. Moreover, despite of the development of the new short-term insulin preparations, intra-patient variability in the insulin pharmacokinetics has not been avoided yet [66, 135].

In this scenario of uncertainty, introducing in the control algorithms models that take uncertainty into account seems to be a good idea.

In Chapter 4, the SMRC algorithm was used mainly to impose constraints to the IOB in order to avoid high values of residual insulin leading to hypoglycemia. The constraints were not applied to the actual and inaccessible IOB values but to an estimation of them. This estimation was performed 
using an insulin model [100] with constant population parameters. Neither intra nor inter-patient variability was considered there. In this chapter, an interval version of the IOB model is used instead. In this way, uncertainty in the parameters of the model is taken into account adding robustness with respect to variability to SAFE algorithm. Depending on the glycemic state of the patient at each instant of time, a more conservative solution or a more aggressive solution is selected. The rules used to determine witch envelope to take are based on a Mandami-type fuzzy model.

\subsection{Theoretical framework}

In this work, the insulin absorption model used for method description is the same used in Chapter 4, the one developed by Cobelli's group [100] (see Section 1.4.2. Refer to Figure 4.4 for a compartmental representation of the model.

$$
\begin{aligned}
& \dot{S}_{1}(t)=-\left(k_{a 1}+k_{d}\right) S_{1}(t)+u(t) \\
& \dot{S}_{2}(t)=k_{d} S_{1}(t)-k_{a 2} S_{2}(t)
\end{aligned}
$$

Uncertainty in the parameters of the model is considered using a monotone systems approach for the computation of IOB envelopes [40]. The advantages of this approach compared to modal interval analysis [26] used in Chapter 3 is that uncertainty can be considered in all the parameters of the model (even in the initial conditions) and that no time-discretisation is required, reducing computational time. This is specially critical in closed-loop systems.

In this section the approach used to study the monotonicity of the system and to compute the output bounds is explained.

\subsubsection{Monotonicity analysis}

Graph theory has been used to perform the monotonicity analysis of the IOB system. In order to include the parameters in the analysis they are considered constant states $\left(\dot{k}_{a 1}=\dot{k}_{a 2}=\dot{k}_{d}=0\right)$. 
By using the species-reaction graph [150] and assigning a node for each state, an activation arrow between two nodes $\mathrm{A}$ and $\mathrm{B}$ represents that the derivative $\partial B / \partial A$ is strictly positive, while an inhibition line denotes that it is strictly negative. If the derivative is equal to zero no line is drawn (no effect). Once the activation and inhibition lines are drawn, nodes connected by an activation arrow will have the same sign (cooperative), whereas nodes connected by an inhibition line will have different sign (competitive). A dynamical system is monotone if there exists at least one consistent assignment. In Figure 5.1 an example of a monotone and non-monotone system is presented.
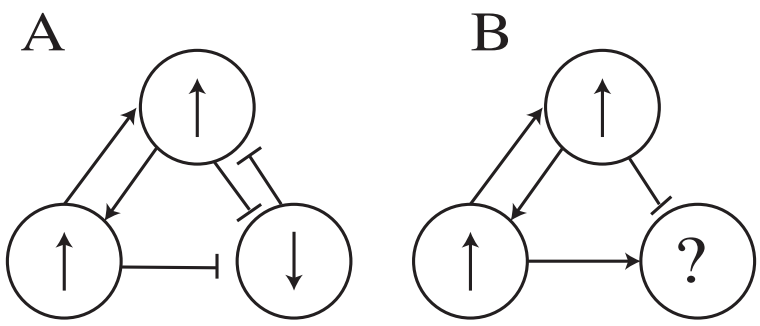

Figure 5.1: Example of a monotone (A) and a non-monotone system (B) [41].

After the monotonicity analysis has been performed, the upper bound is computed taking the maximum values of the cooperative states and the minimum values of the competitive ones. In the same way for the lower bound, the minimum values of the cooperative states and the maximum of the competitive ones are used. The problem arises with non-monotone compartments which need to be considered as intervals. These interval uncertainties produce an overestimation on the computation of output bounds. To solve this issue the solution proposed in [41] has been used here. It consists in combining the equations of the original model to obtain a new model in which the output compartment is unaltered and which is monotone with respect to all the compartments and parameters of the model. Therefore, the computation of its output bounds can be performed without overestimation.

A monotonicity analysis performed to the model used for IOB estimation system (5.1) reveals that the system is not monotone. It is cooperative with respect to the compartments and the parameters $k_{a 1}$ and $k_{a 2}$ are competitive. 
Nevertheless the system is not monotone with respect to parameter $k_{d}$. The partial derivatives of the two compartments with respect to $k_{d}$ take different signs:

$$
\begin{gathered}
\frac{\partial \dot{S}_{1}(t)}{\partial k_{d}}=-S_{1}(t)<0 \\
\frac{\partial \dot{S}_{2}(t)}{\partial k_{d}}=S_{1}(t)>0
\end{gathered}
$$

However, if the equations of the model are combined by performing a change of variables as suggested in [41] the new model to study its monotonicity is:

$$
\begin{aligned}
& \dot{Y}_{1}(t)=-\left(k_{a 1}+k_{d}\right) Y_{1}(t)+u(t) \\
& \dot{Y}_{2}(t)=-k_{a 1} Y_{1}(t)-k_{a 2}\left(Y_{2}(t)-Y_{1}(t)\right)+u(t)
\end{aligned}
$$

where $Y_{1}=S_{1}, Y_{2}=S_{1}+S_{2}$

If we study now the monotonicity of the resulting system:

$$
\begin{aligned}
& \frac{\partial \dot{Y}_{1}(t)}{\partial Y_{2}(t)}=0 ; \quad \frac{\partial \dot{Y}_{1}(t)}{\partial k_{a 1}}=-Y_{1}(t) \leq 0 ; \quad \frac{\partial \dot{Y}_{1}(t)}{\partial k_{a 2}}=0 ; \\
& \frac{\partial \dot{Y}_{1}(t)}{\partial k_{d}}=-Y_{1}(t) \leq 0 ; \quad \frac{\partial \dot{Y}_{1}(t)}{\partial u(t)}=1>0 \\
& \frac{\partial \dot{Y}_{2}(t)}{\partial Y_{1}(t)}=k_{a 2}-k_{a 1} ; \quad \frac{\partial \dot{Y}_{2}(t)}{\partial k_{a 1}}=-Y_{1}(t) \leq 0 ; \\
& \frac{\partial \dot{Y}_{2}(t)}{\partial k_{a 2}}=Y_{1}(t)-Y_{2}(t) \leq 0 ; \quad \frac{\partial \dot{Y}_{2}(t)}{\partial k_{d}}=0 ; \quad \frac{\partial \dot{Y}_{2}(t)}{\partial u(t)}=1>0
\end{aligned}
$$

Therefore, system (5.4) is monotone. If $k_{a 2}-k_{a 1}>0$ the system is cooperative with respect to states $Y_{1}$ and $Y_{2}$ and the insulin input $\mathrm{u}(\mathrm{t})$. Parameters $k_{a 2}, k_{a 1}$ and $k_{d}$ are competitive parameters. Figure 5.2 is a graphical representation of the monotonicity analysis of both system 5.1 and 5.3 .

Figure 5.3 shows the result of the computation of the output bounds of the system using both the traditional monotonicity approach (A) and using 
(A)

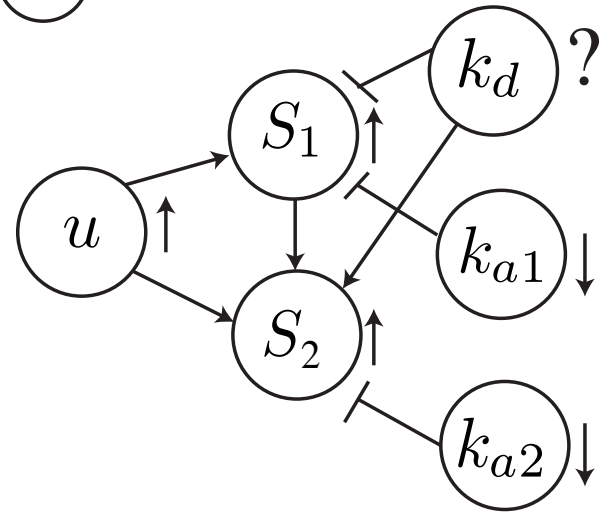

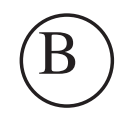

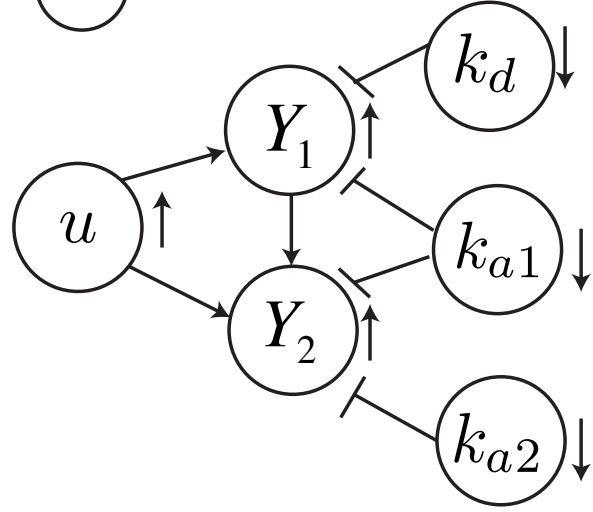

Figure 5.2: Parameters monotonicity of systems 5.1 and 5.3 .

the system (5.3). Red lines represent the computed output bounds while the blue lines are the result of several numerical simulations with variation in the parameters.

In Figure $5.3 \mathrm{~A}$ the original system (5.1) is used. $k_{a 1}$ and $k_{a 3}$ are competitive (minimum value is used for the computation of the upper bound and viceversa for the lower bound ). However both (minimum/maximum) values of the non-monotone paramer $k_{d}$ are considered in the computation of the bounds:

$$
\begin{aligned}
& \left\{\begin{array}{l}
\underline{\dot{S}_{1}(t)}=-\left(\overline{k_{a 1}}+\overline{k_{d}}\right) \underline{S_{1}(t)}+u(t) \\
\underline{\dot{S}_{2}(t)}=\underline{k_{d}} \underline{S_{1}(t)}-\overline{k_{a 2}} \underline{S_{2}(t)}
\end{array}\right. \\
& \left\{\begin{array}{l}
\overline{\dot{S}_{1}(t)}=-\left(\underline{k_{a 1}}+\underline{k_{d}}\right) \overline{S_{1}(t)}+u(t) \\
\overline{\dot{S}_{2}(t)}=\overline{k_{d}} \overline{S_{1}(t)}-\underline{k_{a 2}} \overline{S_{2}(t)}
\end{array}\right.
\end{aligned}
$$

where $\underline{X}$ and $\bar{X}$ represent the lower bound and the upper bound for X. See that the upper bound of $k_{d}$ is used to minimize $S_{1}$ and the upper bound to minimize $S_{2}$. So the standard monotonicity approach ensures the inclusion of 
all the possible solutions, but it produces an overestimation in the computation of solution envelopes.
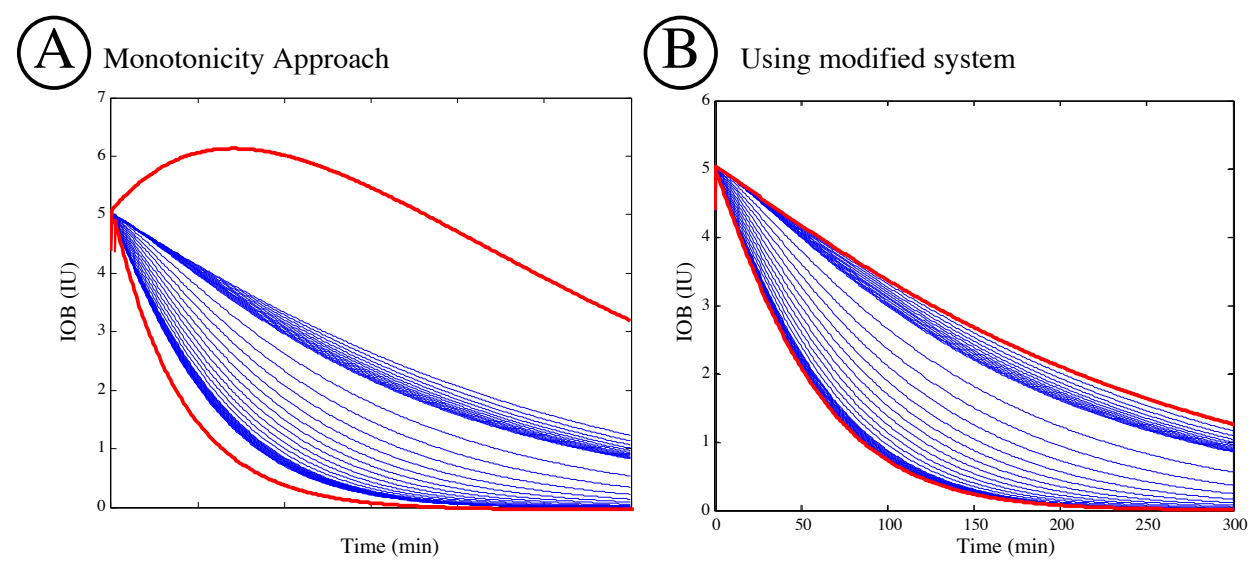

Figure 5.3: Output bounds for the IOB in Cobelli's insulin absorption model developed. $S_{1}(0)=183, S_{2}(0)=194$ and $k_{a 1}=0.0034, k_{a 2}=0.0159, k_{d}=0.0161$ under $60 \%$ uncertainty (worst case in the Cobelli's adult population).

In Figure $5.3 \mathrm{~B}$ however, the monotonicity is studied using system (5.3) which is in fact monotone with respect to all the states and parameters of the model and because of that it can be represented without overestimation.

$$
\begin{aligned}
& \left\{\begin{array}{l}
\underline{\dot{Y}_{1}(t)}=-\left(\overline{k_{a 1}}+\overline{k_{d}}\right) \underline{Y_{1}(t)}+u(t) \\
\underline{\dot{Y}_{2}(t)}=-\overline{k_{a 1}} \underline{Y_{1}(t)}-\overline{k_{a 2}}\left(\underline{Y_{2}(t)}-\underline{Y_{1}(t)}+u(t)\right)
\end{array}\right. \\
& \left\{\begin{array}{l}
\overline{\dot{Y}_{1}(t)}=-\left(\underline{k_{a 1}}+\underline{k_{d}}\right) \overline{Y_{1}(t)}+u(t) \\
\left.\overline{\dot{Y}_{2}(t)}=-\underline{k_{a 1}} \overline{Y_{1}(t)}-\underline{k_{a 2}} \underline{Y_{2}(t)}-\underline{Y_{1}(t)}+u(t)\right)
\end{array}\right.
\end{aligned}
$$

\subsection{Interval version of SAFE}

Uncertainty can be considered in several ways when estimating IOB. If the parameters of the model for each of the patients are unknown a general model with population parameters (like the one used in Chapter 4 can be used but 
considering uncertainty in the parameters to represent inter-patient variability. In case the parameters of each of the patients can be estimated prior of the closed loop study, a less conservative solution could be achieve considering only uncertainty due to intra-patient variability. In this work, the same model (with population parameters) has been used for all the simulations and uncertainty in the parameters has been considered to counteract inter-patient variability. With the interval representation of the IOB profile it can be used to select the worst possibility among all the possible solution of IOB at each step of the algorithm.

In this way, depending on the deviation from normoglycemia of the patient's glycemic status at a specific moment and on the trend of the measured plasma glucose (slope), the limits on IOB can be more or less restrictive. To implement this, for a fix IOB limit the IOB solution used for computation is different. In case the glycemic state of the patient is hypoglycemic, a more restrictive IOB limit should be applied. This is done by using the upper IOB curve to represent the IOB estimation. However, when the plasma glucose is high, the lower curve of the IOB estimation is used or in other words a less restrictive limitation is applied.

In order to decide which value of IOB estimation to take in each moment, a Mandami-type fuzzy model is used. The model has two inputs, the deviation from normoglycemia $(P G-100 \mathrm{mg} / \mathrm{dL})$ and the slope of the plasma glucose. The first one is defined using five member functions (high_negative, low_negative, normal, low_possitive, high_possitive) in the range of -50 to $120 \mathrm{mg} / \mathrm{dL}$ with 0 meaning absolute normoglycemia. For the glucose slope three member functions (neg_slope, medium_slope, poss_slope) are used with a range of -3 to $5 \mathrm{mg} / \mathrm{dL} * \mathrm{~s}$. These inputs are used to select the IOB solution (output) to be considered in SAFE. Three member functions are used to define this output (low, medium and high). Two-sided composite of two different Gaussian curves are used for the definition of all the membership functions.

The fuzzy rules are defined using the AND fuzzy operation and being more restrictive with hypoglycemia states. The method used for deffuzification is the bisector. 
When the output of the fuzzy system is maximum the upper bound of the IOB curve is used as the input of the SAFE algorithm. In the same way when the the output of the fuzzy system is minimum the lower bound is used. Intermediate values correspond to IOB curves between the upper and lower bound interval with central point $(\overline{I O B}-\underline{I O B}) / 2$.

\subsection{Simulations and results}

In order to illustrate the kind of results that are obtained using an interval IOB model in the additional SAFE loop, again the UVA simulator [88] is used.

In Figure 5.4 an example of teh results obtained during $20 \mathrm{~h}$ with three meals $(40-80-70 \mathrm{~g})$ is presented. Here the glucose profile together with the IOB curve is presented for the three options: PID, PID+SAFE and PID +SAFE intervalar. The use of an interval model for the IOB estimation reduces the exposure to hypoglycemia even more than using the simple version of SAFE.

More detail of the performance of this approach is shown in Figure 5.5 . One point is worth to explain: in this approach the IOB limits, apart from following the general rule explained in Section 4.3.4 and Figure 4.5, become adaptive when the IOB profile is switching between the different solutions inside the IOB interval (to guarantee the sliding mode). In that way, the only situation when the IOB curve can be temporally above the limit is when this limit is abruptly reduced during the late postprandial period. In that case SAFE would contribute to enter again in the allowed region with $w(t)=w^{+}$.

For the in silico evaluation the same clinical protocol (16-daily hours study with 3 meals) that was designed for the evaluation of the simple version of SAFE has been used. Again, intra-patient variability was simulated by superimposing to the nominal parameters of each patient sinusoidal oscillations of $5 \%$ amplitude (except for insulin sensitivity which was 10\%) and $3 \mathrm{~h}$ period. The glucose measures used for the controller were taken from a simulated subcutaneous glucose sensor.

A PID with meal announced tuned as in Table 4.1 was used as main controller. 

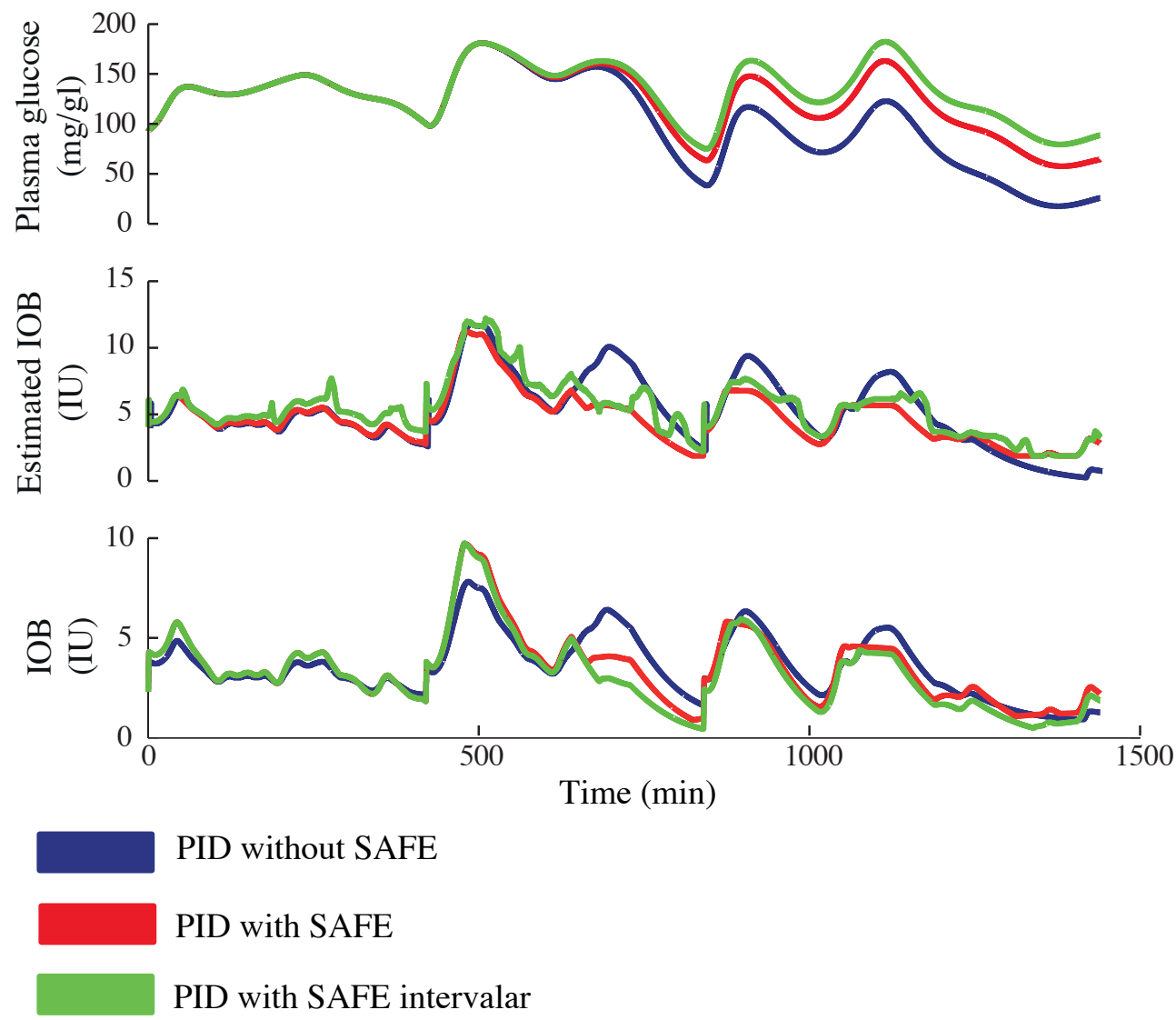

Figure 5.4: Comparison of the performance of the controller in one virtual patient (patient 9) for the 3 options: PID, PID+SAFE and PID +SAFE intervalar loop during one day. Glucose profile and IOB curve (both the IOB estimated and the real IOB profile) are shown. 

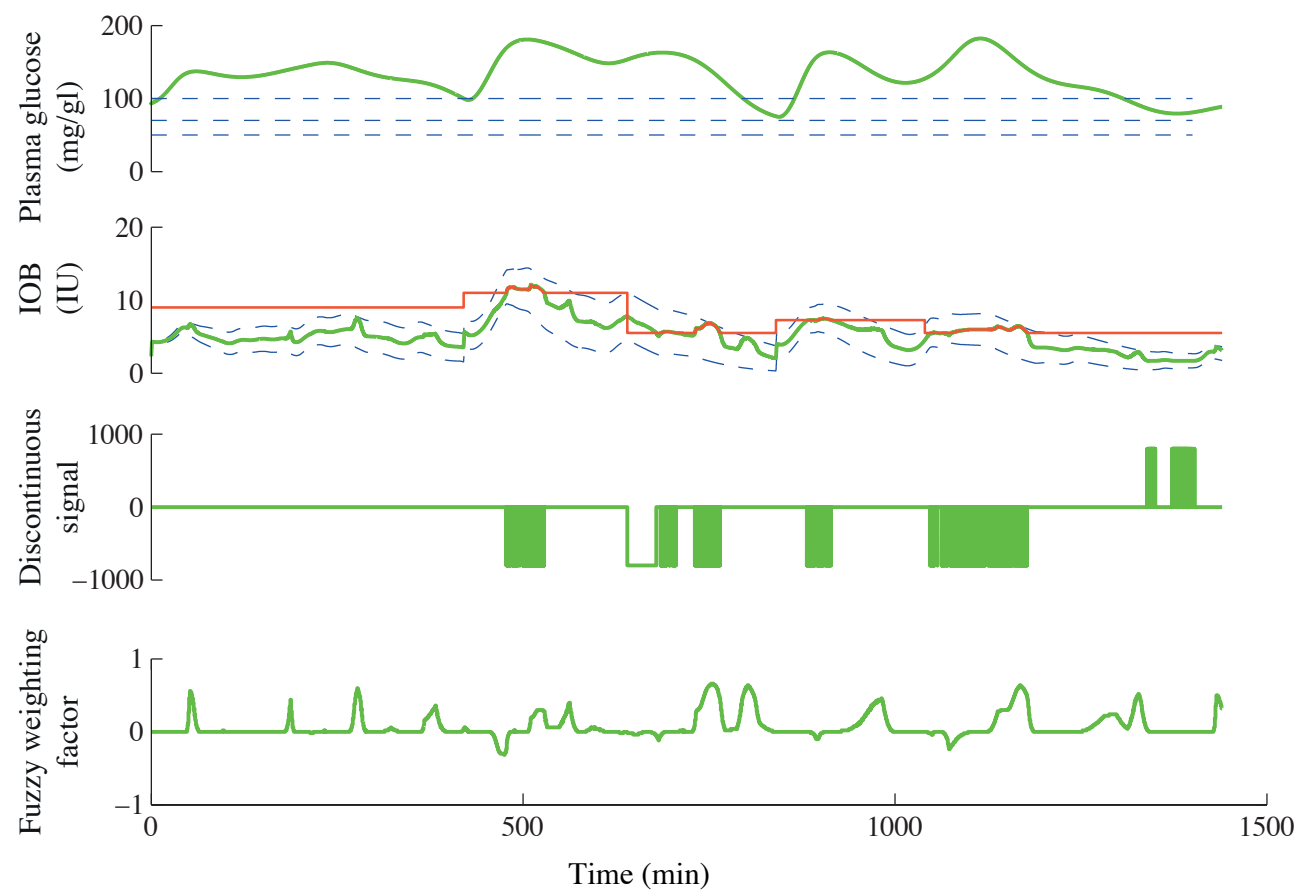

Figure 5.5: Plasma glucose profile, IOB estimation together with the discontinuous signal and weighting coefficient that resulted from the fuzzy system for the PID + the interval version of SAFE. The dotted lines in the IOB curve represent the outer and the inner bounds of the IOB estimation. Note that according to the weighting coefficient the IOB curve used in the algorithm is closer to one or the other bound. 
The rules for choosing the different postprandial upper limits for IOB of this study were the same as the ones used in Chapter 4, being the specific values used specified in Table 4.2. As it was done during the evaluation of the simple version of SAFE the limits were slightly modified depending on the initial conditions (initial conditions of hypo/hyper glycemia implied a $10 \%$ decrease/increase in the IOB limit. In the same way the limits were reduced if they were caused by the second peak of the meal absorption (later postprandial period). As it has been already explained the limits become adaptive during the transition from one possible IOB solution to the another one. A fixed, and proportional to the basal rate of each patient, lower IOB bound was also established.

In Table 5.1 a set of indicators are provided comparing the performance of a controller with a standard PID, the addition of a simple SAFE loop and with the interval version of SAFE. Again normoglycemia is defined as $70 \mathrm{mg} / \mathrm{dl}<$ $P G<140 \mathrm{mg} / \mathrm{dl}$. All data are subjected to repeated-measures analysis of variance with HuynhâFeldt adjustment for nonsphericity [180]. The analysis of variance model included only the test condition (standard PID, PID +SAFE and PID + interval version of SAFE), as within-subjects factor, and post hoc comparisons (Bonferroni test) were carried out to pinpoint specific differences on significant interaction terms.

Note that the numbers shown here for the standard PID and the PID + SAFE solution are not exactly the same as in Table4.3. Thie cause for this behaviour is the consideration of of intra-patient variability, which makes each study unique in spite of being carried out under the same conditions.

The reduction in hypoglycemic exposure is even more notable with this version of SAFE: percentage of time bellow $70 \mathrm{mg} / \mathrm{dl}$ is reduced almost $50 \%$ (4 p.p) with respect the standard SAFE approach ( $8.55 \%$ vs $4.4 \%, p=0.005$ ) and almost $75 \%$ (12 p.p) with respect the PID without safe (16.52\% vs 4.4 $\%, p<0.001)$. Although the increment in hyperglycemia exposure is also statically significant the percentage of time above $180 \mathrm{mg} / \mathrm{dl}$ is only $1.2 \mathrm{p} . \mathrm{p}$ higher than the PID without $\operatorname{SAFE}(2.35 \%$ vs $3.55 \%, p=0.035)$.

Figure 5.6 is a representation of the percentage of time each of the patients 
Table 5.1: Different indicators for the evaluation of interval SAFE

\begin{tabular}{lccccccc}
\hline & $P G<50(\%)$ & $50<P G<70(\%)$ & $P G>180(\%)$ & $140<P G<180(\%)$ & Normo $(\%)$ & Severe & Moderate \\
Total & & & & & hypo events & hypo events \\
\hline Without SAFE & 3.81 & 13.00 & 2.36 & 9.67 & 71.24 & 45 & 96 \\
With SAFE & 0.93 & $7.57^{a}$ & 2.61 & 13.41 & $75.48^{a}$ & $15^{a}$ & $78^{a}$ \\
With interval SAFE & $0.47^{a}$ & $3.97^{a, b}$ & $3.55^{a, b}$ & $15.47^{a, b}$ & $76.53^{a}$ & $8^{a, b}$ & $60^{a, b}$ \\
p value & 0.05 & 0.001 & 0.031 & 0.008 & 0.017 & 0.008 & 0.009 \\
\hline${ }^{a} p<0.05$ versus standard PI & & & & & & \\
${ }^{b} p<0.05$ versus SAFE &
\end{tabular}

spends in hypoglycemia $(P G<70 \mathrm{mg} / \mathrm{dl})$. The reduction of the hypoglycemia exposure is notable in all the cases, being close or greater than $50 \%$ with respect to the standard SAFE algorithm in most of the patients. In Figure 5.7 the percentage of time in each specific glucose range defined in Table 5.1 is also shown. Note that patient 6 is especially difficult to control with high exposure to both hypo and hyperglycemia. However using the interval version of SAFE the percentage of time in normoglycemia is increased 5 p.p with respect the standard PID (53.33\% vs $58.50 \%)$.

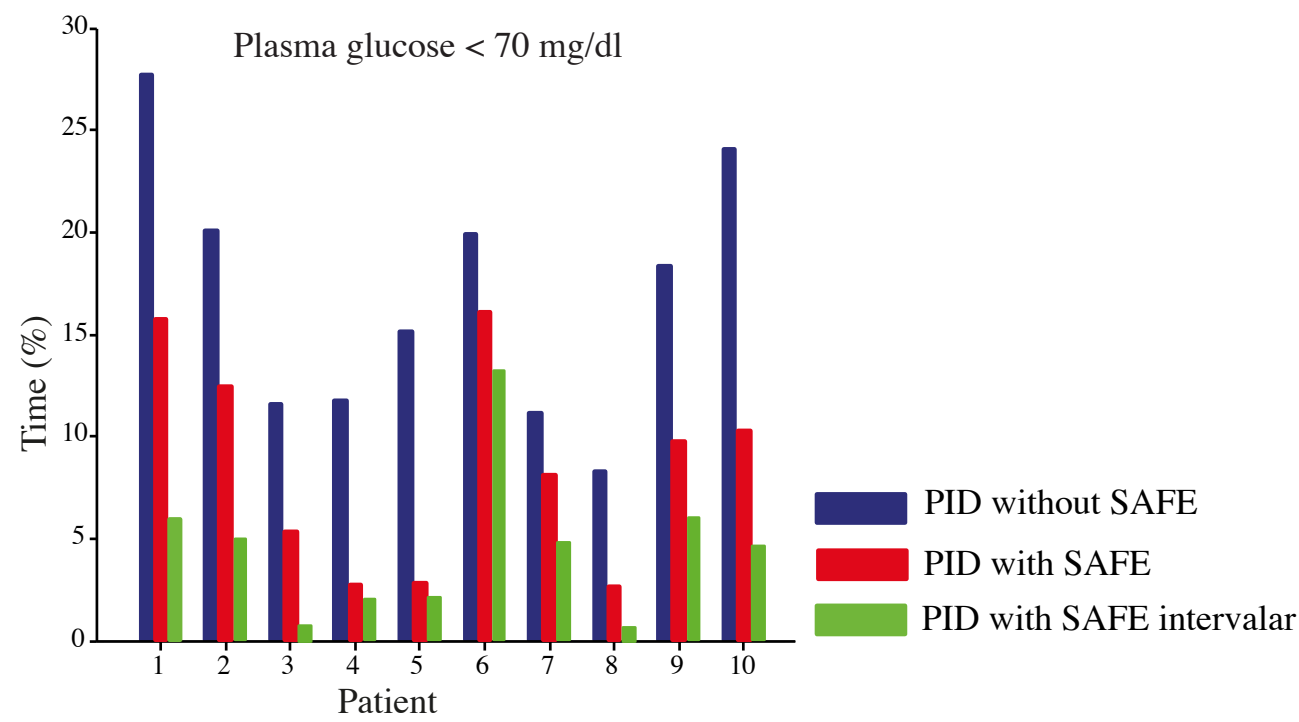

Figure 5.6: Total percentage of time in hypoglycemia $P G<70$ of each patient with the three strategies 

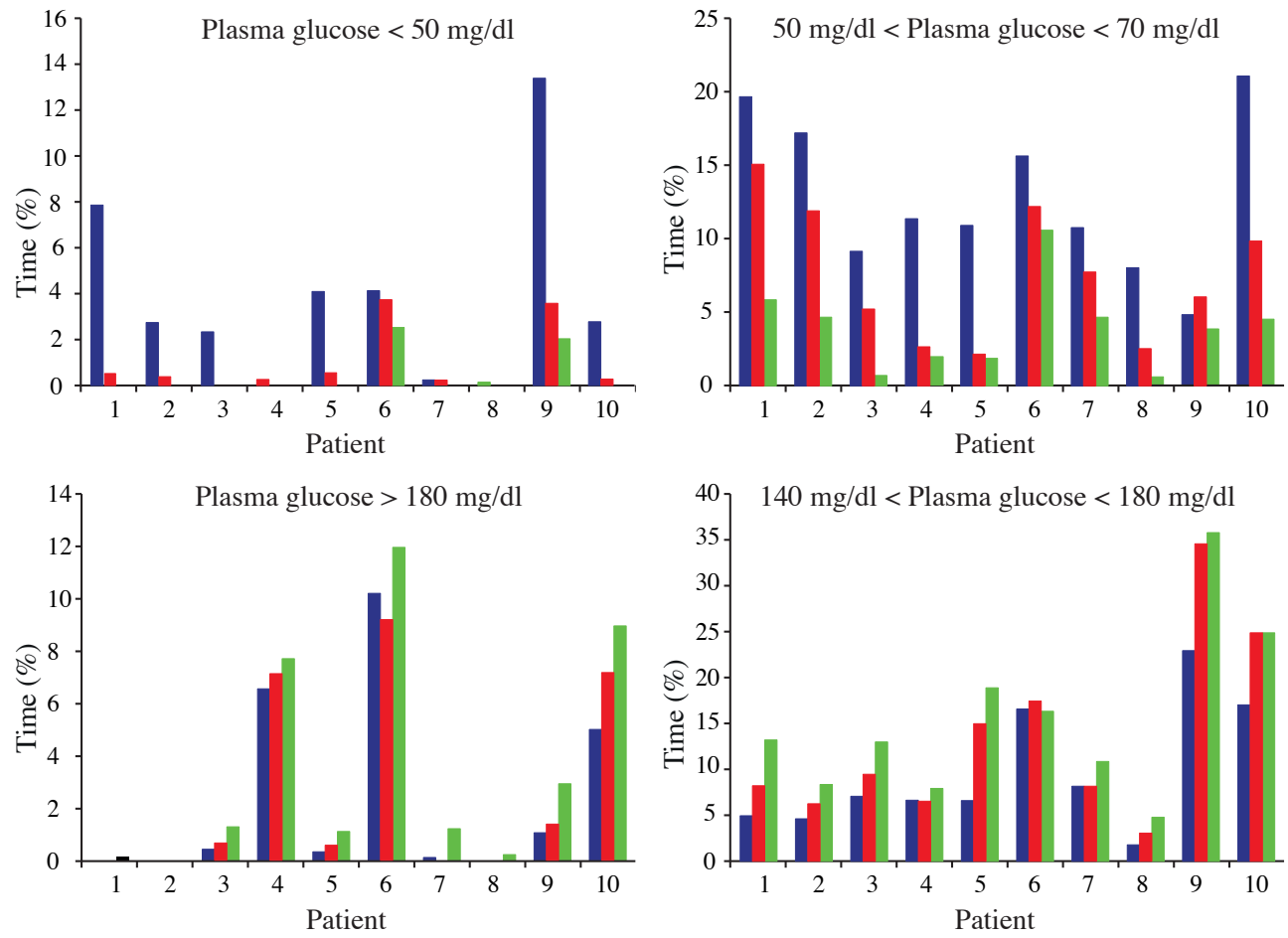

PID without SAFE

PID with SAFE

PID with SAFE intervalar

Figure 5.7: Percentage of time in hypo and hyperglycemia of each patient with standard PID, with the additional SAFE loop and with the version of SAFE using interval IOB. 


\subsection{Conclusions}

A modified version of SAFE algorithm has been presented in this chapter. Taking advance of the monotonicity analysis techniques, uncertainty is considered in the estimation of the IOB. In this manner, the model representing IOB is capable of taking into account both intra and inter-patient variability. This interval model provides the possibility of choosing one or other IOB solution depending on the glycemic state of the patient. For states with hypoglycemic risk SAFE activates when the IOB upper bound is about to violate the constraints. In other situations less restrictive IOB solutions are used. A Mandami-type fuzzy model has been used to decide the specific IOB solution at each simulation step.

An in silico evaluation with the same conditions as in Chapter 4 has also been presented, showing the improvements in the indicators, mainly in those related to hypoglycemia exposure. 


\section{$6 \quad$ Conclusions}

The main aim of this work was to find strategies to improve the performance of current therapies applied to type 1 Diabetes Mellitus. In spite of the development of new types of insulin analogs, the improvements of glucose sensors (specially CGMS) and the use of smart insulin pumps, variability is still the main challenge avoiding the success of both open loop and closed loop therapies. The effects of variability and uncertainty are especially significant during the postprandial period.

Two main strategies have been presented in this document that help in overcoming the main difficulties found in glucose control.

In this way, the first result presented in this thesis was a robust openloop strategy for glucose control in patients with type 1 diabetes using CSII therapy. The algorithm, based on set inversion via interval analysis, provides for a given meal, a set of solutions (basal-bolus) that are designed to maintain the patient in a pre-specified glycemic range (according to the patient's model). The solution can lead to different administration modes: standard, square, dual-wave boluses or temporal basal decrement mode.

One of the strengths of this strategy is that it can consider uncertainty in the models' parameters such as insulin sensitivity or the amount of ingested carbohydrates. Moreover it provides the possibility to determine, in a nonheuristic way, the feasible insulin administration modes for a given meal, which could be included in smarter insulin pumps in the future.

The algorithm showed promising results in the in silico validation using UVa Simulator that were partially confirmed in the in vivo validation. 
The main limitation found in the clinical validation of this proposal was "unexplained" variability, which makes it very difficult to control postprandial glucose response in open loop for some patients, whatever the model used for insulin dosing: the bolus advisors currently implemented in clinical practice, or new proposals.

Closed-loop approaches on the other hand, despite of its advantages with respect to open-loop (perturbation counteraction and behavior against variability) are finding in over-correction and subsequent hypoglycemia in postprandial control the main difficulty. Intra-patient variability, errors in the glucose sensor measurements, and, mainly, the delay in the control action are the problems that need to be overcome. Moreover, an aggressive tuning of the controller and changes in the patients' sensitivity to insulin are additional factors contributing to hypoglycemia.

These facts motivated the second proposal of this thesis: an algorithm, called SAFE, to be added to any glucose closed-loop controller to provide an additional safety layer against hypoglycemias. The algorithm makes use of SMRC to impose limits to the residual insulin activity. SAFE is only active when IOB is about to violate any previously defined constraint. Otherwise, the SAFE loop is inactive and the original control system is not altered at all. In this way, the approach exploits the attributes of the sliding regime as a transitional mode of operation. The extended version of SAFE considers uncertainty in the metabolic parameters to deal with variability. The algorithm demonstrated its robustness with respect perturbations (errors in the meal content estimation, errors in the CGMS...) both theoretically and through simulations.

A relevant advantage of this proposal is that, because of its nature (it uses a two-step control approach for dealing with constraints, is an universal solution that is applicable regardless of the control scheme chosen. Thus, all the knowhow on classical control and tuning techniques can be used for the main control loop design which could be designed previously and in an independent way.

The results of the in silico evaluation (using UVa simulator) showed a significant reduction of hypoglycemia exposure encouraging a clinical valid- 
ation. In fact, Clinical trials to evaluate the efficacy of SAFE are ongoing (NCT02100488). SAFE algorithm has also lead to other in silico studies with multiple controller tuning combinations [97].

\subsection{Publications}

This work has given rise to several publications in journals:

- A. Revert, P. Rossetti, R. Calm. J. Vehí, J. Bondia, Combining Basal- Bolus Insulin Infusion for Tight Postprandial Glucose Control. An In Silico Evaluation in Adults, Children and Adolescents, Journal of Diabetes Science and Technology,(6), 1424-1437, 2010

- A. Revert, R. Calm, J. Vehí, J. Bondia, Calculation of the best basal- bolus combination for postprandial glucose control in insulin pump therapy, IEEE Transactions on Biomedical Engineering,58(2), 274-281, 2011

- P. Rossetti, F.J. Ampudia-Blasco, A. Laguna, A. Revert, J. Vehí, J.F. Ascaso, J. Bondia. Evaluation of a novel continuous glucose monitoring- based method for mealtime insulin dosing - the iBolus in subjects with type 1 diabetes using continuous subcutaneous insulin infusion therapy: a randomized controlled trial, Diabetes Technology and Therapeutics, 14(11), 1043-1052, 2012

- P. Rossetti P, J. Vehí, A. Revert, R. Calm, J. Bondia. Commentary on "Performance of a glucose meter with a built-in automated bolus calculator versus manual bolus calculation in insulin-using subjects" Journal of Diabetes Science and Technology. 2012;6(2):345-7.

- Revert, F. Garelli, J. Pico, H. De Battista, P. Rossetti, J. Vehi, J. Bondia. Safety Auxiliary Feedback Element for the Artificial Pancreas in Type 1 Diabetes, IEEE Transactions on Biomedical Engineering , 60(8), 2113- 2122, 2013 
This research has also been exposed in the folowing congresses:

- A. Revert, P. Rossetti, R. Calm, J. Vehí, J. Bondia. Combining basal-bolus insulin infusion for tight postpandial glucose control. An insilico validation in adults, children and adolescents, 3rd International Conference on Advance Technologies \& Treatments for Diabetes, Basel, Switzerland, 2010.

- A. Revert, R. Calm, J. Vehí, J. Bondia Calculation of the best basal-bolus combination for postprandial glucose in insulin pump therapy, 9th Diabetes Technology Meeting, San Francisco, USA, 2009.

- P. Rossetti, J. Ampudia-Blasco, A. Laguna, A. Revert, J. Vehi, R. Calm, S. Correa, G. Viguer, J. Ascaso, J. Bondia. Cálculo del bolus prandial utilizando monitorización continua de la glucosa en pacientes con diabetes mellitus tipo 1 tratados con infusión subcutánea continua de insulina. XXII Congreso Nacional de la Sociedad Española de Diabetes. Málaga 2011

- Ampudia-Blasco, A. Laguna, A. Revert, J. Vehí, R. Calm, J. F. Ascaso, J. Bondia. A Novel Strategy for Non-Empirical Calculation of Prandial Insulin Bolus Based on Continuous Glucose Monitoring (CGM) in Subjects with Type 1 Diabetes (T1DM) Treated with Continuous Subcutaneous Insulin Infusion (CSII). American diabetes association 71th scientific sessions. 2011

- A Revert, J. Picó, H. De Battista, P. Rossetti, J. Vehí, J. Bondia. A reference conditioning method for the reduction of hypoglycemia in closed loop glucose control. 5th International Conference on Advanced Technologies \& Treatments for Diabetes. Barcelona 2012

- A. Revert, J. Picó, J.Bondia. Control de biosistemas sujetos a restricciones. X Simposio CEA Ingeniería de Control . ETSEIB, UPC, Barcelona 2012. ISBN 978-84-615-7398-1 
- A. Revert, J. Picó, J.Bondia. Estrategias robustas para el control de glucosa en diabéticos tipo 1. XI Simposio CEA de Ingeniería de Control. Universitat Politècnica de Valencia, Valencia 2013. ISBN - 978-84-6957298-6 



\section{References}

[1] Bringing the artificial pancreas home., howpublished $=$ http://www . apathome.eu, note $=$ Accessed: 13-mar-15.

[2] JDRF Artificial Pancreas Project Consortium, howpublished $=$ http: //jdrfconsortium.jaeb.org/viewpage.aspx?pagename=home, note = Accessed: 13-mar-15.

[3] A. Abu-Rmileh, W. Garcia-Gabin, and D. Zambrano. A robust sliding mode controller with internal model for closed-loop artificial pancreas. Medical \& Biological Engineering \& Computing, 48(12):1191-1201, July 2010.

[4] AHD editors. The American Heritage Medical Dictionary. Houghton Mifflin Company, 3rd edition, 2007.

[5] A. M. Albisser, B. S. Leibel, T. G. Ewart, Z. Davidovac, C. K. Botz, W. Zingg, H. Schipper, and R. Gander. Clinical control of diabetes by the artificial pancreas. Diabetes, 23(5):397-404, 1974.

[6] G. Alefeld and J. Herzberger. Interval analysis. Academic Press, 1983.

[7] H. Amann. Ordinary differential equations. Walter de Gruyter, Berlin, 1st edition, 1990.

[8] H. Anhalt and N. J. Bohannon. Insulin patch pumps: their development and future in closed-loop systems. Diabetes technology $\&$ therapeutics, 12(S1):S-51-S-58, 2010. 
[9] A. D. Association. Executive summary: standards of medical care in diabetes-2010. Diabetes Care, 33(supplement 1):S4-S10, 2010.

[10] E. Atlas, R. Nimri, S. Miller, E. A. Grunberg, and M. Phillip. MD-Logic Artificial Pancreas System: A pilot study in adults with type 1 diabetes. Diabetes Care, 33(5):1072-1076, Apr. 2010.

[11] F. Barceló-Rico. Multimodel Approaches for Plasma Glucose Estimation in Continuous Glucose Monitoring. Development of New Calibration Algorithms. $\mathrm{PhD}$ thesis, 2012.

[12] F. Barceló-Rico, J. L. Díez, P. Rossetti, J. Vehí, and J. Bondia. Adaptive Calibration Algorithm for Plasma Glucose Estimation in Continuous Glucose Monitoring. IEEE Journal of Biomedical and Health Informatics, 17(3):530-538, June 2013.

[13] R. Basu, B. Di Camillo, G. Toffolo, A. Basu, P. Shah, A. Vella, R. Rizza, and C. Cobelli. Use of a novel triple-tracer approach to assess postprandial glucose metabolism. Am J Physiol Endocrinol Metab, 284(1):E55-69, 2003.

[14] K. L. Becker, C. R. Kahn, and R. R. W. Principles and Practices of Endocrinology and Metabolism. Lippincott Williams and Wilkins Publishers, 2002.

[15] B. W. Bequette. Challenges and recent progress in the development of a closed-loop artificial pancreas. Annual reviews in control, 36(2):255-266, Dec. 2012.

[16] M. Berger and D. Rodbard. Computer simulation of plasma insulin and glucose dynamics after subcutaneous insulin injection. Diabetes Care, 12(10):725-736, 1989.

[17] M. Bliss and R. Purkis. The discovery of insulin. University of Chicago Press Chicago, 1982. 
[18] G. Boden, X. Chen, R. A. DeSantis, and Z. Kendrick. Effects of Age and Body Fat Insulin Resistance in Healthy Men. Diabetes Care, 16(5):728733, 1993.

[19] E. A. Boland, M. Grey, A. Oesterle, L. Fredrickson, and W. V. Tamborlane. Continuous subcutaneous insulin infusion. A new way to lower risk of severe hypoglycemia, improve metabolic control, and enhance coping in adolescents with type 1 diabetes. Diabetes Care, 22(11):1779-1784, 1999.

[20] J. Bondia, E. Dassau, H. Zisser, R. Calm, J. Vehí, L. Jovanovič, and F. J. Doyle III. Coordinated basal-bolus infusion for tighter postprandial glucose control in insulin pump therapy. Journal of Diabetes Science and Technology, 3(1):89, 2009.

[21] J. Bondia, A. Laguna, P. Rossetti, F. J. Ampudia-Blasco, and J. Vehí. On the use of hard/soft specifications to deal with intra-patient variability in postprandial glucose control in type 1 diabetes. In World Congress, pages 8347-8353, 2011.

[22] J. Bondia, J. Vehí, C. C. Palerm, and P. Herrero. El páncreas artificial: control automático de infusión de insulina en diabetes mellitus tipo 1. Revista Iberoamericana de Automática e Informàtica industrial, 7(2):520, 2010.

[23] M. Breton, A. Farret, D. Bruttomesso, S. Anderson, L. Magni, S. Patek, C. Dalla Man, J. Place, S. Demartini, and S. Del Favero. Fully Integrated Artificial Pancreas in Type 1 Diabetes Modular Closed-Loop Glucose Control Maintains Near Normoglycemia. Diabetes, 61(9):2230-2237, 2012.

[24] D. Bruttomesso, A. Farret, S. Costa, M. C. Marescotti, M. Vettore, A. Avogaro, A. Tiengo, C. Dalla Man, J. Place, and A. Facchinetti. Closed-loop artificial pancreas using subcutaneous glucose sensing and insulin delivery and a model predictive control algorithm: preliminary 
studies in Padova and Montpellier. J Diabetes Sci Technol, 3(5):10141021, 2009.

[25] B. Buckingham, H. P. Chase, E. Dassau, E. Cobry, P. Clinton, V. Gage, K. Caswell, J. Wilkinson, F. Cameron, H. Lee, B. W. Bequette, and F. J. Doyle. Prevention of Nocturnal Hypoglycemia Using Predictive Alarm Algorithms and Insulin Pump Suspension. Diabetes Care, 33(5):10131017, Apr. 2010.

[26] R. Calm, M. García-Jaramillo, J. Bondia, M. A. Sainz, and J. Vehí. Comparison of interval and Monte Carlo simulation for the prediction of postprandial glucose under uncertainty in type 1 diabetes mellitus. Computer Methods and Programs in Biomedicine, pages 1-8, Sept. 2010.

[27] F. Cameron, B. W. Bequette, D. M. Wilson, B. A. Buckingham, H. Lee, and G. Niemeyer. A closed-loop artificial pancreas based on risk management. Journal of Diabetes Science and Technology, 5(2):368, 2011.

[28] D. U. Campos-Delgado, M. Hernandez-Ordonez, R. Femat, and A. Gordillo-Moscoso. Fuzzy-based controller for glucose regulation in type-1 diabetic patients by subcutaneous route. IEEE Transactions on Biomedical Engineering, 53(11):2201-2210, 2006.

[29] J. R. Castle, J. M. Engle, J. E. Youssef, R. G. Massoud, K. C. J. Yuen, R. Kagan, and W. K. Ward. Novel Use of Glucagon in a Closed-Loop System for Prevention of Hypoglycemia in Type 1 Diabetes. Diabetes Care, 33(6):1282-1287, May 2010.

[30] A. Caumo, R. N. Bergman, and C. Cobelli. Insulin sensitivity from meal tolerance tests in normal subjects: a minimal model index. Journal of Clinical Endocrinology \&3 Metabolism, 85(11):4396, 2000.

[31] H. P. Chase, S. Z. Saib, T. MacKenzie, M. M. Hansen, and S. K. Garg. Post-prandial glucose excursions following four methods of bolus insulin administration in subjects with Type 1 diabetes. Diabetic Medicine, 19(4):317-321, 2002. 
[32] H. P. Chase, S. Z. Saib, T. MacKenzie, M. M. Hansen, and S. K. Garg. Postprandial glucose excursions following four methods of bolus insulin administration in subjects with Type 1 diabetes. Diabetic Medicine, 19(4):317-321, 2002.

[33] P. Choudhary, J. Shin, Y. Wang, M. L. Evans, P. J. Hammond, D. Kerr, J. A. M. Shaw, J. C. Pickup, and S. A. Amiel. Insulin Pump Therapy With Automated Insulin Suspension in Response to Hypoglycemia: Reduction in nocturnal hypoglycemia in those at greatest risk. Diabetes Care, 34(9):2023-2025, Aug. 2011.

[34] W. L. Clarke, S. Anderson, M. Breton, S. Patek, L. Kashmer, and B. Kovatchev. Closed-loop artificial pancreas using subcutaneous glucose sensing and insulin delivery and a model predictive control algorithm: the Virginia experience. J Diabetes Sci Technol, 3(5):1031-1038, 2009.

[35] A. H. Clemens, P. H. Chang, and R. W. Myers. The development of Biostator, a Glucose Controlled Insulin Infusion System (GCIIS). Horm Metab Res, Suppl 7:23-33, Jan. 1977.

[36] C. Cobelli, E. Renard, B. P. Kovatchev, P. Keith-Hynes, N. Ben Brahim, J. Place, S. Del Favero, M. Breton, A. Farret, D. Bruttomesso, E. Dassau, H. Zisser, F. J. Doyle, S. D. Patek, and A. Avogaro. Pilot Studies of Wearable Outpatient Artificial Pancreas in Type 1 Diabetes. Diabetes Care, 35(9):e65-e67, Aug. 2012.

[37] C. Dalla Man, M. Camilleri, and C. Cobelli. A System Model of Oral Glucose Absorption: Validation on Gold Standard Data. Biomedical Engineering, IEEE Transactions on, 53(12):2472-2478, June 2006.

[38] C. Dalla Man, R. A. Rizza, and C. Cobelli. Meal Simulation Model of the Glucose-Insulin System. Biomedical Engineering, IEEE Transactions on, 54(10):1740-1749, June 2007.

[39] A. De Leiva-Hidalgo, E. Bruguès-Bruguès, and A. de Leiva-Pérez. From pancreatic extracts to artificial pancreas: History, science and 
controversies about the discovery of the pancreatic antidiabetic hormone. Avances en diabetología, 27(1):27-38, 2011.

[40] D. de Pereda, S. Romero-Vivo, B. Ricarte, and J. Bondia. On the prediction of glucose concentration under intra-patient variability in type 1 diabetes: A monotone systems approach. Computer Methods and Programs in Biomedicine, 108(3):993-1001, Dec. 2012.

[41] D. de Pereda, S. Romero-Vivo, B. Ricarte, and J. Bondia. Guaranteed computation methods for compartmental in-series models under uncertainty. Computers \&3 Mathematics with Applications, 66(9):1595$1605,2013$.

[42] S. Del Favero, A. Facchinetti, G. Sparacino, and C. Cobelli. Improving accuracy and precision of glucose sensor profiles: retrospective fitting by constrained deconvolution. Biomedical Engineering, IEEE Transactions on, 61(4):1044-1053, 2014.

[43] D. E. DeWitt and I. B. Hirsch. Outpatient Insulin Therapy in Type 1 and Type 2 Diabetes Mellitus: Scientific Review. JAMA, 289(17):2254-2264, May 2003.

[44] E. A. Doyle, S. A. Weinzimer, A. T. Steffen, J. A. H. Ahern, M. Vincent, and W. V. Tamborlane. A randomized, prospective trial comparing the efficacy of continuous subcutaneous insulin infusion with multiple daily injections using insulin glargine. Diabetes Care, 27(7):1554-1558, 2004.

[45] C. Edwards and S. K. Spurgeon. Sliding mode control: theory and applications, volume 7. CRC, 1998.

[46] F. H. El-Khatib, S. J. Russell, K. L. Magyar, M. Sinha, K. McKeon, D. M. Nathan, and E. R. Damiano. Autonomous and Continuous Adaptation of a Bihormonal Bionic Pancreas in Adults and Adolescents With Type 1 Diabetes. The Journal of Clinical Endocrinology 8 Metabolism, 99(5):1701-1711, May 2014. 
[47] F. H. El-Khatib, S. J. Russell, D. M. Nathan, R. G. Sutherlin, and E. R. Damiano. A bihormonal closed-loop artificial pancreas for type 1 diabetes. Sci Transl Med, 2(27):27ra27, 2010.

[48] J. El Youssef, J. Castle, and W. K. Ward. A Review of Closed-Loop Algorithms for Glycemic Control in the Treatment of Type 1 Diabetes. Algorithms, 2(1):518-532, Mar. 2009.

[49] D. Elleri, J. Allen, K. Kumareswaran, and L. Leelarathna. Day-andNight Closed-Loop (CL) Glucose Control in Adolescents with Type 1 Diabetes (T1D) . Diabetes, 2011.

[50] D. Elleri, J. M. Allen, M. Biagioni, K. Kumareswaran, L. Leelarathna, K. Caldwell, M. Nodale, M. E. Wilinska, C. L. Acerini, D. B. Dunger, and R. Hovorka. Evaluation of a portable ambulatory prototype for automated overnight closed-loop insulin delivery in young people with type 1 diabetes. Pediatric Diabetes, 13(6):449-453, July 2012.

[51] D. Elleri, J. M. Allen, K. Kumareswaran, L. Leelarathna, M. Nodale, K. Caldwell, P. Cheng, C. Kollman, A. Haidar, H. R. Murphy, M. E. Wilinska, C. L. Acerini, D. B. Dunger, and R. Hovorka. Closed-Loop Basal Insulin Delivery Over 36 Hours in Adolescents With Type 1 Diabetes. June 2013.

[52] C. Ellingsen, E. Dassau, H. Zisser, B. Grosman, M. W. Percival, L. Jovanovič, and F. J. Doyle III. Safety constraints in an artificial pancreatic $\beta$ cell: an implementation of model predictive control with insulin on board. Journal of diabetes science and technology (Online), 3(3):536, 2009.

[53] A. Facchinetti, G. Sparacino, and C. Cobelli. Enhanced Accuracy of Continuous Glucose Monitoring by Online Extended Kalman Filtering. Diabetes technology \& therapeutics, 12(5):353-363, May 2010.

[54] W. Garcia-Gabin, J. Vehı, J. Bondia, C. Tarın, and R. Calm. Robust sliding mode closed-loop glucose control with meal compensation in type 1 diabetes mellitus. pages 4240-4245, 2008. 
[55] M. García-Jaramillo, R. Calm, J. Bondia, C. Tarín, and J. Vehí. Computing the risk of postprandial hypo-and hyperglycemia in type 1 diabetes mellitus considering intrapatient variability and other sources of uncertainty. Journal of Diabetes Science and Technology, 3(4):895, 2009.

[56] F. Garelli, P. Camocardi, and R. J. Mantz. Variable structure strategy to avoid amplitude and rate saturation in pitch control of a wind turbine. International Journal of Hydrogen Energy, 35(11):5869-5875, June 2010.

[57] F. Garelli, L. Gracia, A. Sala, and P. Albertos. Sliding mode speed auto-regulation technique for robotic tracking. Robotics and Autonomous Systems, 59(7):519-529, 2011.

[58] F. Garelli, R. Mantz, and H. De Battista. Limiting interactions in decentralized control of MIMO systems. Journal of Process Control, 16(5):473-483, 2006.

[59] D. E. Goldstein, R. R. Little, R. A. Lorenz, J. I. Malone, D. M. Nathan, C. M. Peterson, and D. B. Sacks. Tests of Glycemia in Diabetes. Diabetes Care, 27(7):1761-1773, 2004.

[60] T. M. Gross, D. Kayne, A. King, C. Rother, and S. Juth. A bolus calculator is an effective means of controlling postprandial glycemia in patients on insulin pump therapy. Diabetes technology 83 therapeutics, 5(3):365-369, 2003.

[61] D. C. Group and C. T. Research. The Diabetes Control and Complications Trial (DCCT) Research Group. The effect of intensive treatment of diabetes on the development and progression of longterm complications in insulin-dependent diabetes mellitus. New England Journal of Medicine, 329:977-986, 1993.

[62] J. E. Hall. Guyton and Hall textbook of medical physiology. Elsevier Health Sciences, 2010.

[63] R. Hanas. Type 1 Diabetes: A Guide for Children, Adolescents, Young 
Adults-and Their Caregivers: Everything You Need to Know to Become an Expert on Your Own Diabetes. DA Capo Press, 2005.

[64] R. Hanus, M. Kinnært, and J. Henrotte. Conditioning technique, a general anti-windup and bumpless transfer method. Automatica, 23(6):729-739, 1987.

[65] E. Haus. Chronobiology in the endocrine system. Advanced Drug Delivery Reviews, 59(9):985-1014, 2007.

[66] L. Heinemann. Variability of insulin absorption and insulin action. Diabetes technology \& therapeutics, 4(5):673-682, 2002.

[67] R. P. L. M. Hoogma, P. J. Hammond, R. Gomis, D. Kerr, D. Bruttomesso, K. P. Bouter, K. J. Wiefels, H. de la Calle, D. H. Schweitzer, M. Pfohl, E. Torlone, L. G. Krinelke, G. B. Bolli, and on behalf of the 5-Nations Study Group1. Comparison of the effects of continuous subcutaneous insulin infusion (CSII) and NPH-based multiple daily insulin injections (MDI) on glycaemic control and quality of life: results of the 5-nations trial. Diabetic Medicine, 23(2):141-147, Feb. 2006.

[68] R. Hovorka. Continuous glucose monitoring and closed-loop systems. Diabetic Medicine, 23(1):1-12, Jan. 2005.

[69] R. Hovorka. The future of continuous glucose monitoring: closed loop. Current Diabetes Reviews, 4(3):269-279, 2008.

[70] R. Hovorka. Closed-loop insulin delivery: from bench to clinical practice. Nature Reviews Endocrinology, 7(7):385-395, Feb. 2011.

[71] R. Hovorka, V. Canonico, L. J. Chassin, U. Haueter, M. MassiBenedetti, M. O. Federici, T. R. Pieber, H. C. Schaller, L. Schaupp, T. Vering, and M. E. Wilinska. Nonlinear model predictive control of glucose concentration in subjects with type 1 diabetes. Physiological Measurement, 25(4):905-920, July 2004. 
[72] R. Hovorka, D. Elleri, H. Thabit, J. M. Allen, L. Leelarathna, R. ElKhairi, K. Kumareswaran, K. Caldwell, P. Calhoun, C. Kollman, H. R. Murphy, C. L. Acerini, M. E. Wilinska, M. Nodale, and D. B. Dunger. Overnight Closed-Loop Insulin Delivery in Young People With Type 1 Diabetes: A Free-Living, Randomized Clinical Trial. Diabetes Care, 37(5):1204-1211, Apr. 2014.

[73] R. Hovorka, J. Kremen, J. Blaha, M. Matias, K. Anderlova, L. Bosanska, T. Roubicek, M. E. Wilinska, L. J. Chassin, S. Svacina, and M. Haluzik. Blood Glucose Control by a Model Predictive Control Algorithm with Variable Sampling Rate Versus a Routine Glucose Management Protocol in Cardiac Surgery Patients: A Randomized Controlled Trial. Journal of Clinical Endocrinology \&3 Metabolism, 92(8):2960-2964, Aug. 2007.

[74] R. Hovorka, F. Shojaee-Moradie, P. V. Carroll, L. J. Chassin, I. J. Gowrie, N. C. Jackson, R. S. Tudor, A. M. Umpleby, and R. H. Jones. Partitioning glucose distribution/transport, disposal, and endogenous production during IVGTT. American Journal of PhysiologyEndocrinology and Metabolism, 282(5):E992-E1007, 2002.

[75] C. S. Hughes, S. D. Patek, M. D. Breton, and B. P. Kovatchev. Hypoglycemia prevention via pump attenuation and red-yellow-green "traffic" lights using continuous glucose monitoring and insulin pump data. Journal of Diabetes Science and Technology, 4(5):1146, 2010.

[76] M. S. Ibbini and M. A. Masadeh. A fuzzy logic based closed-loop control system for blood glucose level regulation in diabetics. Journal of Medical Engineering \{\& Technology, 29(2):64-69, 2005.

[77] International Diabetes Federation Guideline Development Committee. Guideline for management of postmeal glucose. Brussels: International Diabetes Federation, 2007.

[78] L. Jaulin, M. Kieffer, O. Didrit, and E. Walter. Applied Interval Analysis. Springer, 2001. 
[79] S. M. Jones, J. L. Quarry, M. Caldwell-McMillan, D. T. Mauger, and R. A. Gabbay. Optimal insulin pump dosing and postprandial glycemia following a pizza meal using the continuous glucose monitoring system. Diabetes technology \&3 therapeutics, 7(2):233-240, 2005.

[80] A. H. Kadish. Automation control of blood sugar a servomechanism for glucose monitoring and control. ASAIO Journal, 9:363-367, 1963.

[81] D. L. Kasper, A. S. Fauci, D. L. Longo, E. Braunwald, S. L. Hauser, and J. L. Jameson. Harrison Principles of Internal Medicine. McGraw-Hill, 16th edition, 2005.

[82] P. Kaveh and Y. B. Shtessel. Blood glucose regulation using higher-order sliding mode control. International Journal of Robust and Nonlinear Control, 18(4-5):557-569, 2008.

[83] T. Klupa, T. Benbenek-Klupa, M. Malecki, M. Szalecki, and J. Sieradzki. Clinical usefulness of a bolus calculator in maintaining normoglycaemia in active professional patients with type 1 diabetes treated with continuous subcutaneous insulin infusion. The Journal of International Medical Research, 36(5):1112-1116, 2008.

[84] T. Klupa, J. Skupien, K. Cyganek, B. Katra, J. Sieradzki, and M. T. Malecki. The dual-wave bolus feature in type 1 diabetes adult users of insulin pumps. Acta Diabetologica, 48(1):11-14, Jan. 2010.

[85] T. Kobayashi, S. Sawano, T. Itoh, K. Kosaka, H. Hirayama, and Y. Kasuya. The pharmacokinetics of insulin after continuous subcutaneous infusion or bolus subcutaneous injection in diabetic patients. Diabetes, 32(4):331-336, 1983.

[86] M. Kothare, P. Campo, M. Morari, and K. Nett. A unified frammework for the study of anti-windup designs. Automatica, 30(12):1869-1883, 1994.

[87] B. Kovatchev, C. Cobelli, E. Renard, S. Anderson, M. Breton, S. Patek, W. Clarke, D. Bruttomesso, A. Maran, and C. Silvana. Multinational 
study of subcutaneous model-predictive closed-loop control in type 1 diabetes mellitus: summary of the results. Journal of Diabetes Science and Technology, 4(6):1374, 2010.

[88] B. P. Kovatchev, M. Breton, C. Dalla Man, and C. Cobelli. Biosimulation Modeling for Diabetes: In Silico Preclinical Trials: A Proof of Concept in Closed-Loop Control of Type 1 Diabetes. Journal of diabetes science and technology (Online), 3(1):44-55, 2009.

[89] B. P. Kovatchev, P. Keith-Hynes, M. D. Breton, and S. D. Patek. Unified platform for monitoring and control of blood glucose levels in diabetic patients. Technical report, 2012.

[90] A. J. Kowalski. Can we really close the loop and how soon? Accelerating the availability of an artificial pancreas: a roadmap to better diabetes outcomes. Diabetes technology \& therapeutics, 2009.

[91] E. W. Kraegen, D. E. James, A. B. Jenkins, and D. J. Chisholm. Doseresponse curves for in vivo insulin sensitivity in individual tissues in rats. American Journal of Physiology-Endocrinology and Metabolism, 248(3):E353-E362, 1985.

[92] J. Kropff, D. Bruttomesso, W. Doll, A. Farret, S. Galasso, Y. M. Luijf, J. K. Mader, J. Place, F. Boscari, and T. R. Pieber. Accuracy of two continuous glucose monitoring systems: a headtohead comparison under clinical research centre and daily life conditions. Diabetes, Obesity and Metabolism, 2014.

[93] K. Kumareswaran, D. Elleri, J. M. Allen, J. Harris, D. Xing, C. Kollman, M. Nodale, H. R. Murphy, S. A. Amiel, and S. R. Heller. Artificial Pancreas: Meta-Analysis of Overnight Closed-Loop Randomized Studies in Children and Adults with Type 1 Diabetes: The Cambridge Cohort. Journal of Diabetes Science and Technology, 5(6):1352-1362, 2011.

[94] M. Kuure-Kinsey, C. C. Palerm, and B. W. Bequette. A Dual-Rate Kalman Filter for Continuous Glucose Monitoring. pages 1-4, July 2006. 
[95] A. J. Laguna, P. Rossetti, F. J. Ampudia-Blasco, J. Vehí, and J. Bondia. Experimental blood glucose interval identification of patients with type 1 diabetes. Journal of Process Control, 24(1):171-181, 2014.

[96] E. D. Lehmann, C. Tarin, J. Bondia, E. Teufel, and T. Deutsch. Incorporating a Generic Model of Subcutaneous Insulin Absorption into the AIDA v4 Diabetes Simulator: 1. A Prospective Collaborative Development Plan. Journal of diabetes science and technology (Online), 1(3):423, 2007.

[97] F. León-Vargas, F. Garelli, H. De Battista, and J. Vehí. Postprandial response improvement via safety layer in closed-loop blood glucose controllers. Biomedical Signal Processing and Control, 16:80-87, 2015.

[98] J. Li and Y. Kuang. Systemically modeling the dynamics of plasma insulin in subcutaneous injection of insulin analogues for type 1 diabetes. Mathematical Biosciences and Engineering: MBE, 6(1):41-58, 2009.

[99] L. Ljung. System Identification: Theory for the User (2nd Edition). Prentice Hall PTR, Dec. 1999.

[100] L. Magni, D. M. Raimondo, L. Bossi, C. D. Man, G. De Nicolao, B. Kovatchev, and C. Cobelli. Model predictive control of type 1 diabetes: an in silico trial. Journal of Diabetes Science and Technology, 1(6):804?812, 2007.

[101] C. D. Man, F. Micheletto, D. Lv, M. Breton, B. Kovatchev, and C. Cobelli. The UVA/PADOVA Type 1 Diabetes Simulator: New Features. Journal of Diabetes Science and Technology, 8(1):26-34, Jan. 2014.

[102] R. Mantz, H. De Battista, F. Garelli, and F. Bianchi. Novel conditioning technique for systems subjected to contraints. In Proceedings of 8th International Workshop on Variable Structure Systems, 2004.

[103] G. Marchetti, M. Barolo, L. Jovanovič, H. Zisser, and D. E. Seborg. 
A feedforward-feedback glucose control strategy for type 1 diabetes mellitus. Journal of Process Control, 18(2):149-162, Feb. 2008.

[104] J. Mareczek and M. Buss. Preliminary studies on geometric invariance control synthesis. In Proceedings of European Control Conference, 1999.

[105] J. J. Mastrototaro. The MiniMed Continuous Glucose Monitoring System. Diabetes technology \& therapeutics, 2(supplement 1):13-18, Dec. 2000 .

[106] V. C. Medvei. The history of clinical endocrinology: a comprehensive account of endocrinology from earliest times to the present day. CRC Press, 1993.

[107] R. E. Moore. Interval analysis. Prentice-Hall Inc., Englewood Cliffs, N.J., 1966.

[108] M. Morari. Some Control Problems in the Process Industries. In link.springer.com, pages 55-77. Birkhäuser Boston, Boston, MA, 1993.

[109] D. M. Nathan, P. A. Cleary, J. Y. Backlund, S. M. Genuth, J. M. Lachin, T. J. Orchard, P. Raskin, and B. Zinman. Intensive diabetes treatment and cardiovascular disease in patients with type 1 diabetes. The New England Journal of Medicine, 353(25):2643-2653, 2005.

[110] R. Nimri, E. Atlas, M. Ajzensztejn, S. Miller, T. Oron, and M. Phillip. Feasibility Study of Automated Overnight Closed-Loop Glucose Control Under MD-Logic Artificial Pancreas in Patients with Type 1 Diabetes: The DREAM Project. Diabetes technology \& therapeutics, 14(8):728-735, Aug. 2012.

[111] R. Nimri, T. Danne, O. Kordonouri, E. Atlas, N. Bratina, T. Biester, M. Avbelj, S. Miller, I. Muller, M. Phillip, and T. battelino. The "Glucositter" overnight automated closed loop system for type 1 diabetes: A randomized crossover trial . Pediatric Diabetes, 14:159-167, 2013. 
[112] R. Nimri, I. Muller, E. Atlas, S. Miller, O. Kordonouri, N. Bratina, C. Tsioli, M. A. Stefanija, T. Danne, T. battelino, and M. Phillip. Night glucose control with MD-Logic artificial pancreas in home setting: a single blind, randomized crossover trial-interim analysis. Pediatric Diabetes, 15(2):91-99, Aug. 2013.

[113] N. Oliver, P. Georgiou, D. Johnston, and C. Toumazou. A Benchtop Closed-loop System Controlled by a Bio-Inspired Silicon Implementation of the Pancreatic beta Cell. Journal of Diabetes Science and Technology, 3(6):1419, 2009.

[114] C. C. Palerm. Physiologic insulin delivery with insulin feedback: A control systems perspective. Computer Methods and Programs in Biomedicine, 102(2):130-137, May 2011.

[115] E. Pańkowska and M. Błazik. Bolus calculator with nutrition database software, a new concept of prandial insulin programming for pump users. Journal of Diabetes Science and Technology, 4(3):571, 2010.

[116] E. Pańkowska, M. Błazik, and L. Groele. Does the Fat-Protein Meal Increase Postprandial Glucose Level in Type 1 Diabetes Patients on Insulin Pump: The Conclusion of a Randomized Study. Diabetes technology \& therapeutics, 14(1):16-22, Jan. 2012.

[117] R. S. Parker, F. J. Doyle, III, J. H. Ward, and N. A. Peppas. Robust $\mathrm{H}_{\infty}$ glucose control in diabetes using a physiological model. AIChE Journal, 46(12):2537-2549, 2000.

[118] S. D. Patek, L. Magni, E. Dassau, C. Hughes-Karvetski, C. Toffanin, G. De Nicolao, S. Del Favero, M. Breton, C. Man, E. Renard, et al. Modular closed-loop control of diabetes. Biomedical Engineering, IEEE Transactions on, 59(11):2986-2999, 2012.

[119] T. L. Pearson. Advances in Insulin Pen Technologies: Practical Aspects of Insulin Pen Devices. Journal of Diabetes Science and Technology, 4(3):522, 2010. 
[120] A. Penfornis, E. Personeni, and S. Borot. Evolution of Devices in Diabetes Management. Diabetes technology $\&$ therapeutics, 13(S1):S93-S-102, June 2011.

[121] Y. Peng, D. Vrancic, and R. Hanus. Anti-windup, bumpless, and conditioned transfer techniques for pid controllers. Control Systems, IEEE, 16(4):48-57, 1996.

[122] M. W. Percival, Y. Wang, B. Grosman, E. Dassau, H. Zisser, L. Jovanovič, and F. J. Doyle, III. Development of a multi-parametric model predictive control algorithm for insulin delivery in type 1 diabetes mellitus using clinical parameters. Journal of Process Control, 21(3):391404, Mar. 2011.

[123] E. Pfeiffer, C. Thum, and A. Clemens. The Artificial Beta Cell - A Continuous Control of Blood Sugar by External Regulation of Insulin Infusion (Glucose Controlled Insulin Infusion System). Horm Metab Res, 6(05):339-342, Jan. 1974.

[124] M. Phillip, T. battelino, E. Atlas, O. Kordonouri, N. Bratina, S. Miller, T. Biester, M. Avbelj Stefanija, I. Muller, R. Nimri, and T. Danne. Nocturnal Glucose Control with an Artificial Pancreas at a Diabetes Camp. New England Journal of Medicine, 368(9):824-833, Feb. 2013.

[125] J. C. Pickup. Semi-Closed-Loop Insulin Delivery Systems: Early Experience with Low-Glucose Insulin Suspend Pumps. Diabetes technology \& therapeutics, 13(7):695-698, July 2011.

[126] J. C. Pickup, H. Keen, G. C. Viberti, M. C. White, E. M. Kohner, J. A. Parsons, and K. Alerti. Continuous subcutaneous insulin infusion in the treatment of diabetes mellitus. Diabetes Care, 3(2):290-300, 1980.

[127] J. C. Pickup, J. Kidd, S. Burmiston, and N. Yemane. Determinants of glycaemic control in type 1 diabetes during intensified therapy with multiple daily insulin injections or continuous subcutaneous insulin infusion: importance of blood glucose variability. Diabetes/Metabolism Research and Reviews, 22(3):232-237, 2006. 
[128] J. Picó, F. Garelli, H. De Battista, and R. J. Mantz. Geometric invariance and reference conditioning ideas for control of overflow metabolism. Journal of Process Control, 19(10):1617-1626, Dec. 2009.

[129] W. R. Puckett and E. N. Lightfoot. A model for multiple subcutaneous insulin injections developed from individual diabetic patient data. American Journal of Physiology-Endocrinology and Metabolism, 269(6):E1115-E1124, 1995.

[130] G. Quiroz and R. Femat. Theoretical blood glucose control in hyperand hypoglycemic and exercise scenarios by means of an $\mathrm{H}$ [infinity] algorithm. Journal of Theoretical Biology, 263(1):154-160, 2010.

[131] R. E. Moore. Methods and Applications of Interval Analysis. SIAM Studies in Applied and Numerical Mathematics, 1979.

[132] A. Ralston and P. Rabinowitz. A First Course in Numerical Analysis. Dover Publications Inc., 2nd edition, 2003.

[133] H. K. Reiband, S. Schmidt, A. Ranjan, J. J. Holst, S. Madsbad, and K. Nørgaard. Dualhormone treatment with insulin and glucagon in patients with type 1 diabetes mellitus. Diabetes/Metabolism Research and Reviews, 2014.

[134] E. Renard, J. Place, M. Cantwell, H. Chevassus, and C. C. Palerm. Closed-Loop Insulin Delivery Using a Subcutaneous Glucose Sensor and Intraperitoneal Insulin Delivery: Feasibility study testing a new model for the artificial pancreas. Diabetes Care, 33(1):121-127, Dec. 2009.

[135] A. Revert. Modelado e identificación de la absorción subcutánea de la insulina humana y análogos de insulina. Master's thesis, Universitat Politècnica de València, 2008.

[136] A. Revert, R. Calm, J. Vehí, and J. Bondia. Calculation of the Best Basal-Bolus Combination for Postprandial Glucose Control in Insulin Pump Therapy. IEEE Transactions on Biomedical Engineering, 58(2):274-281. 
[137] A. Revert, F. Garelli, J. Picó, H. De Battista, P. Rossetti, J. Vehí, and J. Bondia. Safety auxiliary feedback element for the artificial pancreas in type 1 diabetes. Biomedical Engineering, IEEE Transactions on, 60(8):2113-2122, 2013.

[138] A. Revert, P. Rossetti, R. Calm, J. Vehí, and J. Bondia. Combining Basal-Bolus Insulin Infusion for Tight Postprandial Glucose Control: An in Silico Evaluation in Adults, Children, and Adolescents. Journal of Diabetes Science and Technology, 4(6):1424, 2010.

[139] D. Rodbard. New and improved methods to characterize glycemic variability using continuous glucose monitoring. Diabetes technology $\&$ therapeutics, 11(9):551-565, 2009.

[140] P. Rossetti, F. J. Ampudia-Blasco, A. Laguna, A. Revert, J. Vehí, J. F. Ascaso, and J. Bondia. Evaluation of a Novel Continuous Glucose Monitoring-Based Method for Mealtime Insulin Dosing - the iBolus -in Subjects with Type 1 Diabetes Using Continuous Subcutaneous Insulin Infusion Therapy: A Randomized Controlled Trial. Diabetes technology \& therapeutics, 14(11):1043-1052, Nov. 2012.

[141] P. Rossetti, J. Bondia, J. Vehí, and C. G. Fanelli. Estimating Plasma Glucose from Interstitial Glucose: The Issue of Calibration Algorithms in Commercial Continuous Glucose Monitoring Devices. Sensors, 10(12):10936-10952, Dec. 2010.

[142] P. Rossetti, J. Vehí, A. Revert, R. Calm, and J. Bondia. Commentary on ?performance of a glucose meter with a built-in automated bolus calculator versus manual bolus calculation in insulin-using subjects? Journal of diabetes science and technology, 6(2):345-347, 2012.

[143] E. Ruiz-Velazquez, R. Femat, and D. U. Campos-Delgado. Blood glucose control for type I diabetes mellitus: A robust tracking $\mathrm{H}$ [infinity] problem. Control Engineering Practice, 12(9):1179-1195, 2004. 
[144] S. J. Russell, F. H. El-Khatib, D. M. Nathan, and E. R. Damiano. Efficacy determinants of subcutaneous microdose glucagon during closedloop control. J Diabetes Sci Tech, 4:1288-1304, 2010.

[145] S. J. Russell, F. H. El-Khatib, M. Sinha, K. L. Magyar, K. McKeon, L. G. Goergen, C. Balliro, M. A. Hillard, D. M. Nathan, and E. R. Damiano. Outpatient Glycemic Control with a Bionic Pancreas in Type 1 Diabetes. New England Journal of Medicine, 371(4):313-325, July 2014.

[146] G. Scheiner, R. J. Sobel, D. E. Smith, A. J. Pick, D. Kruger, J. King, and K. Green. Insulin pump therapy guidelines for successful outcomes. The diabetes educator, 35(2 suppl):29S-41S, 2009.

[147] B. Shashaj, E. Busetto, and N. Sulli. Benefits of a bolus calculator in pre- and postprandial glycaemic control and meal flexibility of paediatric patients using continuous subcutaneous insulin infusion (CSII). Diabetic Medicine, 25(9):1036-1042, Aug. 2008.

[148] M. Shichiri. Artificial endocrine pancreas : development and clinical applications. Kamome Press, Kumamoto, 2000.

[149] S. Shimoda, K. Nishida, M. Sakakida, Y. Konno, K. Ichinose, M. Uehara, T. Nowak, and M. Shichiri. Closed-loop subcutaneous insulin infusion algorithm with a short-acting insulin analog for long-term clinical application of a wearable artificial endocrine pancreas. Frontiers of Medical and Biological Engineering, 8(3):197-211, 1997.

[150] E. D. Sontag. Monotone and near-monotone biochemical networks. Systems and Synthetic Biology, 1(2):59-87.

[151] G. M. Steil, C. C. Palerm, N. Kurtz, G. Voskanyan, A. Roy, S. Paz, and F. R. Kandeel. The Effect of Insulin Feedback on Closed Loop Glucose Control. Journal of Clinical Endocrinology $\&$ Metabolism, 96(5):14021408, May 2011.

[152] G. M. Steil, A. E. Panteleon, and K. Rebrin. Closed-loop insulin 
delivery - the path to physiological glucose control. Advanced Drug Delivery Reviews, 56(2):125-144, 2004.

[153] G. M. Steil, K. Rebrin, C. Darwin, F. Hariri, and M. F. Saad. Feasibility of Automating Insulin Delivery for the Treatment of Type 1 Diabetes. Diabetes, 55(12):3344-3350, Dec. 2006.

[154] D. Takahashi, Y. Xiao, and F. Hu. A survey of insulin-dependent diabetes-part II: control methods. International Journal of Telemedicine and Applications, 2008:1-14, 2008.

[155] W. V. Tamborlane, R. S. Sherwin, M. Genel, and P. Felig. Reduction to Normal of Plasma Glucose in Juvenile Diabetes by Subcutaneous Administration of Insulin with a Portable Infusion Pump. New England Journal of Medicine, 300(11):573-578, Mar. 1979.

[156] S. Tarbouriech and M. Turner. Anti-windup design: an overview of some recent advances and open problems. IET Control Theory and Applications, 3(1):1-19, 2009.

[157] C. Tarin, E. Teufel, J. Picó, J. Bondia, and H. J. Pfleiderer. Comprehensive Pharmacokinetic Model of Insulin Glargine and Other Insulin Formulations. Biomedical Engineering, IEEE Transactions on, 52(12):1994-2005, Dec. 2005.

[158] The DCCT Research Group. Epidemiology of severe hypoglycemia in the diabetes control and complications trial. American Journal of Medicine, The, 90(4):450-459, Apr. 1991.

[159] The Expert Committee on the Diagnosis and Classification of Diabetes Mellitus. Report of the expert committee on the diagnosis and classification of diabetes mellitus. Diabetes Care, 20:1183-1197, 1997.

[160] The Juvenile Diabetes Research Foundation Continuous Glucose Monitoring Study Group. Continuous glucose monitoring and intensive treatment of type 1 diabetes. N Engl J Med, 359(14):1464-1476, 2008. 
[161] G. Toffolo, C. D. Man, C. Cobelli, and A. L. Sunehag. Glucose fluxes during OGTT in adolescents assessed by a stable isotope triple tracer method. Journal of Pediatric Endocrinology \& Metabolism: JPEM, 21(1):31-45, 2008.

[162] Z. Trajanoski, P. Wach, P. Kotanko, A. Ott, and F. Skraba. Pharmacokinetic model for the absorption of subcutaneously injected soluble insulin and monomeric insulin analogues. Biomedizinische Technik, 38(9):224-231, 1993.

[163] Y. Tsukamoto, Y. Kinoshita, H. Kitagawa, M. Munekage, E. Munekage, Y. Takezaki, T. Yatabe, K. Yamashita, R. Yamazaki, T. Okabayashi, M. Tarumi, M. Kobayashi, S. Mishina, and K. Hanazaki. Evaluation of a Novel Artificial Pancreas: Closed Loop Glycemic Control System With Continuous Blood Glucose Monitoring. Artificial Organs, 37(4):E67E73, Mar. 2013.

[164] UK Prospective Diabetes Study (UKPDS) Group. Intensive bloodglucose control with sulphonylureas or insulin compared with conventional treatment and risk of complications in patients with type 2 diabetes (UKPDS 33). The Lancet, 352(9131):837-853, 1998.

[165] A. Vignoni, J. Picó, F. Garelli, and H. De Battista. Sliding mode reference conditioning for coordination in swarms of non-identical multiagent systems. In Variable Structure Systems (VSS), 2012 12th International Workshop on, pages 231-236. IEEE, 2012.

[166] R. Visentin, C. Dalla Man, Y. C. Kudva, A. Basu, and C. Cobelli. Circadian Variability of Insulin Sensitivity: Physiological Input for In Silico Artificial Pancreas. Diabetes technology \& therapeutics, 17(1):1-7, 2015.

[167] H. Wahrenberg. Use of waist circumference to predict insulin resistance: retrospective study. BMJ, 330(7504):1363-1364, June 2005.

[168] W. Waldhäusl. Circadian rhythms of insulin needs and actions. Diabetes Research and Clinical Practice, 6(4):S17-S24, 1989. 
[169] K. Walgama and J. Sternby. Conditioning technique for MIMO processes with input saturation. IEE Proceedings on Control Theory and Applications, 140:231-241, 1993.

[170] J. Walsh. Changes in diabetes care: a history of insulin and pumps past, present, and future. Technical report, 2004.

[171] J. Walsh and R. Roberts. Pumping insulin: everything you need for success on a smart insulin pump. Torrey Pines Pr, 2006.

[172] J. Walsh, R. Roberts, and T. Bailey. Guidelines for insulin dosing in continuous subcutaneous insulin infusion using new formulas from a retrospective study of individuals with optimal glucose levels. Journal of Diabetes Science and Technology, 4(5):1174, 2010.

[173] J. Walsh, R. Roberts, and T. Bailey. Guidelines for optimal bolus calculator settings in adults. Journal of Diabetes Science and Technology, 5(1):129, 2011.

[174] E. Walter and L. Pronzato. Identification of Parametric Models: from Experimental Data. Springer, 1 edition, 1997.

[175] S. A. Weinzimer, J. L. Sherr, E. Cengiz, G. Kim, J. L. Ruiz, L. Carria, G. Voskanyan, A. Roy, and W. V. Tamborlane. Effect of Pramlintide on Prandial Glycemic Excursions During Closed-Loop Control in Adolescents and Young Adults With Type 1 Diabetes. Diabetes Care, 35(10):1994-1999, Sept. 2012.

[176] S. A. Weinzimer, G. M. Steil, K. L. Swan, J. Dziura, N. Kurtz, and W. V. Tamborlane. Fully automated closed-loop insulin delivery versus semiautomated hybrid control in pediatric patients with type 1 diabetes using an artificial pancreas. Diabetes Care, 31(5):934-939, 2008.

[177] M. E. Wilinska, L. J. Chassin, C. L. Acerini, J. M. Allen, D. B. Dunger, and R. Hovorka. Simulation environment to evaluate closed-loop insulin delivery systems in type 1 diabetes. Journal of Diabetes Science and Technology, 4(1):132, 2010. 
[178] M. E. Wilinska, L. J. Chassin, H. C. Schaller, L. Schaupp, T. R. Pieber, and R. Hovorka. Insulin Kinetics in Type-1 Diabetes: Continuous and Bolus Delivery of Rapid Acting Insulin. Biomedical Engineering, IEEE Transactions on, 52(1):3-12, Jan. 2005.

[179] G. Williams and J. C. Pickup. Handbook of Diabetes. Blackwell Publishing, 3rd edition, 2004.

[180] B. J. Winer, D. R. Brown, and K. M. Michels. Statistical Principles In Experimental Design. McGraw-Hill Humanities/Social Sciences/Languages, 3 edition, 1991.

[181] J. Wong, J. G. Chase, C. E. Hann, G. M. Shaw, T. F. Lotz, J. Lin, and A. J. Le Compte. A subcutaneous insulin pharmacokinetic model for computer simulation in a diabetes decision support role: validation and simulation. Journal of diabetes science and technology (Online), 2(4):672, 2008.

[182] T. Yatabe, R. Yamazaki, H. Kitagawa, T. Okabayashi, K. Yamashita, K. Hanazaki, and M. Yokoyama. The evaluation of the ability of closedloop glycemic control device to maintain the blood glucose concentration in intensive care unit patients*. Critical Care Medicine, 39(3):575-578, Mar. 2011.

[183] Y. Zheng, H. T. Kreuwel, D. L. Young, L. K. Shoda, S. Ramanujan, K. G. Gadkar, M. A. Atkinson, and C. C. Whiting. The Virtual NOD Mouse. Annals-New York Academy of Sciences, 1103:45, 2007.

[184] H. Zisser, L. Robinson, W. Bevier, E. Dassau, C. Ellingsen, F. J. Doyle III, and L. Jovanovič. Bolus Calculator: A Review of Four "Smart" Insulin Pumps. Diabetes technology \& therapeutics, 10(6):441-444, Dec. 2008. 